\title{
CHROMOSOME RESEARCH
}

\author{
http://www.kluweronline.com/issn/0967-3849
}

Contents

Volume 12 Supplement 1

September 2004

\author{
ICC XV, 15th International Chromosome Conference \\ 5-10 September 2004 \\ Brunel University, West London, UK
}

Edited by: Joanna M. Bridger Darren K. Griffin

\section{Editorial}

Conference Programme

Plenary Session: Lectures L01-L07

Animal Chromosomes Symposium: Lectures L09-L17; Posters PO:06:01-PO:06:15

Centromeres Symposium: Lectures L18-L28; Posters PO:06:16-PO:06:22

Genome Organisation Symposium: Lectures L29-L46; Posters PO:06:23-PO:06:32

Reproduction Symposium: Lectures L47-L54; Posters PO:06:33-PO:06:36

Comparative Genomics/Chromosome Evolution Symposium: Lectures L55-L65;

Posters PO:06:37-PO:06:55

Telomeres Symposium: Lectures L66-L70; Posters PO:06:56-PO:06:64 55

Epigenetics Symposium: Lectures L71-L78; Posters PO:06:65-PO:06:71 61

Plant Chromosomes Symposium: Lectures L79-L90; Posters PO:06:72-PO:06:94 67

Prokaryote Chromosomes Symposium: Lectures L91-L95; Posters PO:08:01-PO:08:02 84

Sex Chromosomes Symposium: Lectures L96-L107; Posters PO:08:03-PO:08:14 87

Chromosome Transfer Symposium: Lectures L108-L111 Posters PO:08:15-PO:08:16 98

Gene Therapy Symposium: Lectures L112-L119; Posters PO:08:17-PO:08:22 101

Meiosis Symposium: Lectures L120-L126; Posters PO:08:23-PO:08:36 107

Population Cytogenetics Symposium: Lectures L127-L137; Posters PO:08:37-PO:08:53 117

Solid Tumours Symposium: Lectures L138-L147; Posters PO:08:54-PO:08:64 128

Aneuploidy Symposium: Lectures L148-L155; Posters PO:08:65-PO:08:77 137

New Methodologies Symposium: Lectures L156-L165; Posters PO:08:78-PO:08:83 146

Haematological Malignancy Symposium: Lectures L166-L174; Posters PO:08:84-PO:08:94 152

Post-genomic Era Symposium: Lectures L175-L180 161

Prize Nominee Special Symposium: Lectures L181-L191 163

Author Index $\quad 171$

Important Notes and Reminders for Authors inside back cover 


\title{
CHROMOSOME RESEARCH
}

\author{
Cytogenetics, Genomics, Chromatin and The Nucleus
}

\section{FRONT COVER PICTURE}

3D reconstruction of a mouse fibroblast (NIH3T3) nucleus after visualization of telomeres by 3D-FISH and DNA counterstaining. The border of the nucleus (red outside, grey inside) was reconstructed using TO-PRO-3 counterstain and then partly removed to show the inside with reconstructed telomere signals (green). Segmentation of the nucleoli (yellow) was performed using the counterstain labelling. Maximum intensity projections (XY, XZ, YZ) of false-coloured image stacks are shown on the background. Laser confocal serial sections were recorded by a confocal microscope (Leica TCS SP) with a voxel size $=0.05 \times 0.05 \times 0.250 \mu \mathrm{m}$. Reconstruction was performed by surface rendering using software Amira ${ }^{\mathrm{TM}}$ (Amira 3.0, TGS Europe, Merignac, France; http://www.amiravis.com). FISH experiment and 3D reconstruction were performed in the laboratory of Prof. T. Cremer by A. Brero, Department of Biology II, University of Munich, Germany.

Chromosome Research is covered in Biochemistry \& Biophysics Citation Index, Biological Abstracts, BIOSIS Previews, Biotechnology Citation Index, Chemical Abstracts, Current Contents/Life Sciences, Elsevier BIOBASE/Current Awareness in Biological Sciences, EMBASE/Excerpta Medica, IBIDS, Index Medicus/MEDLINE, The ISI Alerting Services, Science Citation Index, Science Citation Index Expanded, and in CD-ROM via Adonis.

\section{SUBSCRIPTION INFORMATION}

Chromosome Research is published 8 times a year (2004).

Subscription prices year 2004 (Volume 12, 8 issues) including postage and handling: EUR 1248.00/USD 1248.00 (print or electronic access), EUR 1497.60/USD 1497.60 (print and electronic access).

Personal price per volume: EUR 350.00 /USD 350.00 including postage and handling.

Subscriptions should be sent to Kluwer Academic Publishers Group, PO Box 322, 3300 AH Dordrecht, The Netherlands, or at PO Box 358, Accord Station, Hingham, MA 02018-0358, USA, or to any subscription agent.

For advertisement rates, prices of back volumes, and other information, please apply to Kluwer Academic Publishers, PO Box 17, 3300 AA Dordrecht, The Netherlands. Fax: (+31)-78-6183273.

Photocopying. In the USA: This journal is registered at the Copyright Clearance Center, Inc., 222 Rosewood Drive, Danvers, MA 01923

Authorisation to photocopy items for internal or personal use, or the internal or personal use of specific clients, is granted by Kluwer Academic Publishers, for users registered with the Copyright Clearance Center (CCC). The "services" for users can be found on the internet at 〈www.copyright.com〉. For those organisations that have been granted a photocopy licence, a separate system of payment has been arranged. Authorisation does not extend to other kinds of copying, such as that for general distribution, for advertising or promotional purposes, for creating new collective works, or for resale.

In the rest of the world: Permission to photocopy must be obtained from the copyright holder. Please apply to Kluwer Academic Publishers, PO Box 17,3300 AA Dordrecht, The Netherlands.

Chromosome Research is published monthly except for March, June, September and December.

Subscription price, per volume: USD 1248.00

Periodicals postage paid at Rahway, NJ 07001. ISSN 9067-3849.

US Mailing Agent: Mercury Airfreight International Ltd., 365 Blair Road, Avenel, NJ 07001.

Published by Kluwer Academic Publishers, Van Godewijckstraat 30, 3311 GX Dordrecht, The Netherlands, and 101 Philip Drive, Norwell, MA 02061, USA. Postmaster: please send all address corrections to: Chromosome Research, c/o Mercury Airfreight International Ltd., 365 Blair Road, Avenel, NJ 07001, USA.

Consent to publish in this journal entails the author's irrevocable and exclusive authorisation of the publisher to collect any sums or considerations for copying or reproduction payable by third parties (as mentioned in article 17, paragraph 2, of the Dutch Copyright Act of 1912 and in the Royal Decree of June 20, 1974 (S.351) pursuant to article 16b of the Dutch Copyright Act of 1912) and/or to act in or out of court in connection herewith.

Printed on acid-free paper

(C) 2004 Kluwer Academic Publishers. Printed in the Netherlands 


\section{ICC XV}

\section{$1^{\text {th }}$ International Chromosome Conference}

September 5-10, 2004; Brunel University, London, UK

\section{CONFERENCE ORGANIZING COMMITTEES}

\section{Local Organizers}

Hosts: Joanna Bridger and Darren Griffin (Cell and Chromosome Biology Group, Brunel University)

Zac Peake (Cambridge Publications)

Jackie Storey (Cambridge Publications)

Charmaine Grimes (Brunel Conferencing Services)

Shiela Killen (Brunel Conferencing Services)

\section{Standing Committee of the International Chromosome Conferences}

Hans de Jong (Wageningen, The Netherlands)

Peter Moens (Toronto, Canada)

Ettore Olmo (Ancona, Italy)

Maria Puertas (Madrid, Spain)

Jeremy Searle (York, UK)

Adrian Sumner (North Berwick, UK) - Chair

Dwayne Wise (Mississippi, USA)

\section{International Advisory Board}

Martin Bentz (Ulm, Germany)

Adrian Bird (Edinburgh, UK)

Nigel Carter (Cambridge, UK)

Titia De-Lange (New York, USA)

Joy Delhanty (London, UK)

Bill Earnshaw (Edinburgh, UK)

Malcolm Ferguson-Smith (Cambridge, UK)

Terry Hassold (Cleveland, USA)

Pat Heslop-Harrison (Leicester, UK)

Pat Hunt (Cleveland, USA)

Clare Huxley (London, UK)

Harold Klinger (New York, USA)

Peter Lichter (Heidelberg, Germany)

Richard Losick (Harvard, USA)

Jennifer Marshall-Graves (Canberra, Australia)

Rob Newbold (Brunel University, UK)

Michael Schmid (Wuerzburg, Germany)

Jeremy Searle (York, UK)

Kevin Sullivan (La Jolla, USA)

Hans Tanke (Leiden, Netherlands)

Andrew Wright (Boston, USA) 



\section{Editorial}

\section{ICC XV, 15th International Chromosome Conference \\ 5-10 September 2004, Brunel University}

This issue of Chromosome Research contains the abstracts of ICC XV, the 15th International Chromosome Conference held at Brunel University on 5-10 September 2004. The International Chromosome Conferences began in 1964, 1967 and 1970 as the Oxford Chromosome Conferences. They became "International" at the fourth conference in Jerusalem in 1972 and a tri-annual format has continued more or less thereafter with conferences in Leiden (1974), Helsinki (1977), Oxford (1980), Lubeck (1983), Marseille (1986), Uppsala (1989), Edinburgh (1992), Madrid (1995), Ancona (1998) and Wurzburg (2001). The year 2004 sees a return to the UK and a first chance for Brunel University to host the ICC. The decision to hold ICC XV at Brunel was initiated in a Franconian wine cellar at The Residence in Wurzburg and we'd like to think that we were awarded it because of our conference facilities and proximity to Heathrow Airport. Delegates will benefit from being only 15-20 minutes away from their arrival and departure gates and Brunel will house the majority of delegates on campus for the duration of the conference. Brunel University is known locally for its landscaped gardens and "brutalist" architecture; we have over 850 academic staff and over 12,000 students. Delegates from Great Britain will be aware of Isambard Kingdom Brunel, the great Victorian engineer after whom the University is named, leading the "greatest Briton" poll for the majority of the long-running saga before being usurped by Winston Churchill at last minute.

We were delighted that, following our initial approaches, our "wish-list" of speakers was largely fulfilled. We have attracted large contingents from the US, Asia and Australasia and there are around 120 invited speakers. This volume contains most of the abstracts of their presentations.
We were also overwhelmed by the response of our would-be presenters at the conference; around 250 new abstracts were received (in addition to those of our invited speakers) and approximately 100 of these have been selected as oral presentations. In addition there will be two poster sessions, collectively comprising around 150 posters accompanied by our commercial exhibition space. We hope to have over 700 delegates, each contributing to the unique nature of ICC. Our intention is to create an atmosphere of the highest quality science and the opportunity for delegates to discuss new projects and ideas in a relaxed environment. It is our belief that the best science is not always done in the lecture rooms but in the bars (i.e. The Chromosome Arms, specially renamed for the conference) and dining rooms of conference centres. The International Chromosome Conferences have always promoted the social side of conferencing as well as the science and ICC XV will be no exception. At ICC $\mathrm{XV}$ there will be visits to the West End, Windsor Castle, Virginia Water, the Tate Modern Gallery and the London Eye. Events on-campus will include real ale tasting, a FISH and chip supper, film shows and a musical recital and - for the conference dinner - a medieval banquet, with traditional dancing. We would like to express our appreciation for the efforts of senior management at Brunel for listening to our pleas when the campus building work threatened to impede our progress, to Brunel Conferencing Services for arranging the room bookings and catering and, especially to Cambridge Publications for their organisational and management skills.

Each day will follow a similar theme with sessions starting about 9 a.m. We will favour the "continental style" of lunch break with sessions winding up around noon and re-starting about 2.30 p.m. The reason for this is to allow time for all delegates to have a decent lunch and to allow adequate time for the viewing of posters and exhibitions. We would also encourage as much social interaction and scientific discussions and 
are willing to provide rooms for that purpose if required.

\section{Day 1}

Monday 6 December will see the whole conference gathered together for the keynote and plenary lectures. Our keynote speaker will be Professor John Burn of the University of Newcastle. Professor Burn is the Public Orator for Newcastle University and is well known for his television appearances and futuristic vision. We look forward with great enthusiasm to his aptly entitled presentation "The Future of Genetics."

Chromosome research is fortunate to have had three eras that have laid down fundamentals for biological and biomedical studies; our first plenary session will celebrate this. The first, the classical era, began with the discovery of the correct number of human chromosomes and the correlations between numerical aberrations and disease (such as Down syndrome and Klinefelter syndrome). Our plenary speaker to represent this era, Professor Pat Jacobs, was involved in the discovery of the genetic basis of what we now know as sex chromosome abnormalities and her activities continue to this day. Prof. Jacobs will not only discuss numerical abnormalities of the karyotype but other chromosome abnormalities such as fragile $\mathrm{X}$ that led to the discovery of trinucleotide repeat expansions in disease. The ability to band human chromosomes brought chromosome research into a second era, enabling the detection of subtle rearrangements and heralding the advent of prenatal diagnosis and cancer cytogenetics. The name that is most synonymous with chromosome banding is Dr Adrian Sumner who also happens to be chair of the standing committee for ICC. Dr Sumner will discuss chromosome banding pre- and post-1968 and inform the delegates on the usefulness of banding for chromosome identification, referencing the position of particular sequences and in diagnostics. Finally, the decision about whom to represent the renaissance of chromosome research, namely the FISH era, led us to the name of Professor David Ward. In a time when molecular biologists were largely writing the obituaries for cytogenetics, the technique of fluorescence in situ hybridization (FISH) burst on the scene and silenced the doubters. Prof. Ward will inform the conference about the advancements in FISH and how these have helped move cytogenetics into the 21 st century.

We would like to take the opportunity of ICC $\mathrm{XV}$ to propose that we are now entering the fourth era of chromosome research, namely that of chromosome engineering. The Brunellian overtones have not escaped us but we would argue that they are well founded given the extensive interest in gene therapy, gene function, genome organization and chromosome transfer, all of which derive their origins from chromosome research. Therefore our second plenary session will be themed on chromosome engineering. We are delighted to have attracted some of the world leaders in the field of chromosome engineering interested in the development and utility of human and mammalian artificial chromosomes. They are Professor Andy Choo of Melbourne Australia, Dr Christine Farr of the University of Cambridge UK and Professor Huntington F. Willard of Duke University USA. Dr Farr and Professor Willard are pioneers of the "top down" and "bottom up" construction strategies for mammalian artificial chromosomes, respectively. In addition, Professor Choo's laboratory were the first to prove the existence of human neocentromeres, regions of chromosomes that form functional centromeres without alpha satellite DNA and use them to create artificial chromosomes. Prof. Willard will inform the conference about behavioural and functional properties of de novo constructed human artificial chromosomes. The properties of "top-down" constructed mammalian artificial chromosomes will be discussed by Dr Farr, with respect to what is required of a minichromosome-based vector system in vertebrate cells and aspects of centromere structure and function in them. Prof. Choo will talk about many aspects of neocentromeres, from why they may exist to revealing centromeric fundamentals, the extent of matrix attachment regions in them and their protein binding properties.

The day will conclude with a reception sponsored by Chromosome Research then a traditional English FISH and chip supper with British real ale tasting. All will be accompanied by live traditional jazz. All subsequent days will begin with the opportunities for young scientists (PhD 
students and post-docs) to meet, and breakfast with, their scientific heroes at "Mentor Breakfasts."

\section{Day 2}

Tuesday 7 September will see the beginning of the concurrent sessions. The largest lecture room is devoted to genome organization which describes how the chromosomes are positioned and function in their main environment, the cell nucleus. This is a fast-growing and complex area of genome/chromosome biology and it is becoming apparent how important creating the right micro-environment for particular sequences of DNA could be in terms of epigenetics, control of transcription, repair and health. This session is chaired by Kevin Sullivan, who has done some elegant work using GFP to study elements of genome movement and organization in real-time. Speaking in this session is Wendy Bickmore, whose research has been instrumental in deciphering how the genome is arranged in cell nuclei. She will talk about her recent research on distribution and organization of open and condensed regions of chromatin throughout the genome. Susan Gasser, will inform delegates about her latest work defining a higher-order compaction of chromatin within yeast nuclei and by using sophisticated live imaging techniques how her group have mapped the organization of yeast interphase chromosomes in 3 dimensions. Thomas Cremer, one of the world leaders in terms of understanding the 3-dimensional organisation of chromosomes and nuclei, will present data supporting the view that chromosomes are organized in the interphase nucleus according to their size. He will also discuss his gene relocation hypothesis, i.e. when genes become silenced during differentiation they can translocate to new regions of the nucleus (this promises to be a most colourful talk). Tom Misteli will tell us about his exciting new data on how genome organization is dissimilar in different tissue types and how this may be linked to the prevalence of specific tissue-related translocations. Andrew Belmont will discuss the exquisite molecular tools he uses to decipher how chromatin is folded and organized in the interphase nucleus. Joanna
Bridger will present her data on genome and health, specifically in diseases of the nucleus and in differentiating adult stem cells.

Also included on this day will be the sessions on centromeres and telomeres led by Bill Earnshaw and Titia De-Lange, respectively (both of whom are figureheads in their respective fields); no self-respecting chromosome conference could do without sessions on these most fundamental of structures. Speakers in the centromere session are Iain Cheeseman who will tell us about new proteins discovered at the C. elegans kinetochore, Stephen Taylor who will demonstrate that Bub1 and Aurora act together to bind the anaphase promoting complex in human cells, Tatsou Fukagawa will describe how the centromere/ kinetochore is assembled and functions by using DT40 cells to knockout specific genes, Beth Sullivan will describe the unique organization of histone modification found at centromeres, Poala Vagnerilli will discuss aspects of centromere function and Alison Pidoux will speak from Robin Allshire's laboratory on centromere/kinetochore function in fission yeast. Bill Earnshaw, as chair, will impart the discovery, using proteomics, of a new chromosomal scaffold protein. The telomere session contains the following speakers Julie Cooper who will tell us about aspects of telomere function in fission yeast, Predrag Slijepcevic will discuss his studies on DNA damage response on telomere maintenance, Woodring Wright will show us how the shortest telomeres co-localize with DNA damage and reveal temporal issues concerning the replication timing of specific telomeric ends and the negative regulation of telomerase by POT1 will be demonstrated, in addition to quantification of telomere length at the nucleotide level in yeast by Joachim Lingner.

The day also includes sessions on animal chromosomes and comparative genomics (led by Michael Schmid and Malcolm Ferguson-Smith respectively) as well as afternoon sessions from our selected abstracts in the topics of plant chromosomes and new methodologies. In animal chromosomes we will hear about the chicken karyotype from Darren Griffin, a genetic map for rabbit from Helene Hayes, Ross Jones will enlighten us about hybrid zone analysis correlated with geographical distribution in shrews and Helen Foster will describe nuclear organization of 
the pig genome in different cell types. The comparative genomic session is also related to chromosome evolution and is chaired by Malcolm Ferguson-Smith, and he will be discussing the role of cross-species chromosome painting in comparative and evolutionary genomics. The talks will be given by Stefan Mueller on primate chromosomes, and Dave Burt will discuss the resource chickNET containing large amounts of sequence information on the chicken genome and how this can be used in evolution studies. We are also looking forward to hearing from Stephen O'Brien about his recent advances in comparative genomics. The Reproduction session is headed by Joy Delhanty, an expert on reproductive cytogenetics, who will discuss her latest data and use of technology in using FISH and comparative genome hybridization to look for aneuploidies in oocytes and polar bodies. Leeanda Wilton will inform us on her efforts to use single cell CGH in aneuploidy screens in IVF embryos and Maj Hultén will discuss aspects of male infertility related meiotic abnormalities. Meiotic segregation of Robertsonian and reciprocal translocations in sperm and pre-implantation embryos will be presented by Elvire van Assche. Finally Helen Tempest will discuss the prospects for the efficacy of alternative herbal therapy in the treatment of high levels of sperm aneuploidy in infertile men.

The day will conclude with a classical music recital and wine reception.

\section{Day 3}

Wednesday 8 September will be half devoted to science and half to social events. The topics of sex chromosomes, plant chromosomes, prokaryotic chromosomes, chromosome transfer and epigenetics will all grace the lecture halls. Invited chairs are Jennifer Marshall-Graves, Pat HeslopHarrison, Andrew Wright, Harold Klinger and Adrian Bird. We are particularly excited to introduce a session on structure and organization of bacterial chromosomes which shows the rapid development of this field in recent years. The chair, Andrew Wright, will show us how fluorescence-based methods can be used to study bacterial DNA and how F plasmid segregation is facilitated in $E$. coli, Jeff Errington will demon- strate chromosome segregation in Bacillus subtilis, Remus Dame will speak on DNA compaction in E. coli and Dave Sherrat will talk about bacterial chromosome segregation. The ICC has always been a bastion for researchers in plant chromosomes and this year a large contingent of scientists studying plant chromosomes will be present. The chair of this session, Pat Heslop-Harrison is well known for his pioneering work on the cytogenetics of plant chromosomes which has been recognized all over the world. In this session, Andreas Houben will discuss histone H3 phosphorylation in plants, Jiming Jiang will tell us about aspects of centromeres in rice, whereas Minoru Murata will tell us about centromeres in Arabidopsis, Paul Fransz will talk about the consequences of a paracentric inversion in Arabidopsis. This session will also complement that of the previous day where the selected abstracts from the plant chromosome session will be given the opportunity to speak.

Chromosome transfer is a technique that was initially used to isolate individual chromosomes from the rest of the karyotype and now has a wide range of applications. The chromosome transfer session will concentrate on the diverse way that chromosome transfer has been incorporated into chromosome biology to study genome organization (Karen Meaburn), for chromosome engineering (Mitsou Oshimura) and to study tumour suppressor genes (Christopher Parris and Harold Klinger).

Epigenetics is an extremely important topic in chromosome biology, since it concerns the modifications to and influences on DNA that affect chromosome function, that are not directly to do with DNA sequence. The epigenetics session, chaired by Adrian Bird, a world-leader in this topic, will contain talks on the effect of histone modifications in disease such as cancer (Tony Kouzarides), the fine mapping of epigenetic domains in an individual gene cluster (Douglas Higgs), silencing of chromosomal regions in plants (Alan Herr) and aspects of epigenetic alterations in nuclear transfer embryos (Petra Hajkova). We are delighted to welcome some of the world leaders in sex chromosome research led by Jenny Graves. Evolution, X-inactivation, Y chromosome deletions, ectopic homologous recombination event, genome organization and 
the avian $\mathrm{Z}$ chromosome will all be covered. Paul Burgoyne, Carolyn Brown, Heather McQueen, Julian Lange, Horst Hameister, Dimitri Filatov and Mike Mitchell, among others, will all be giving presentations. In the afternoon delegates will have a choice of a journey: East into the centre of London to visit the Tate Modern Gallery, the London Eye and the Theatre District; or West to Virginia Water and Windsor.

\section{Day 4}

Our final day of concurrent sessions (Thursday 9 September) will consist of presentations on gene therapy, post genomic era, new methodologies and population cytogenetics. The gene therapy session, chaired by Clare Huxley, will comprise some more talks on the engineering of mammalian artificial chromosomes from Brenda Grimes, William Brown, Zoia Larin-Monaco and Hiroshi Masumoto. George Dickson will describe his outstanding recent work on using gene therapy to add or correct genes in vivo. This session is full of abstracts on chromosome engineering so the talks selected from the abstracts should be especially exciting. The post-genomic era session is chaired by Nigel Carter who will present advances in DNA microarray used to understand structure and function within the human genome. Celia May will demonstrate the use of SNP-class DNA markers to understand meiotic recombination and Eric Green will talk about studying human genome function via multi-species comparisons. Bryan Young will speak about his research on looking for novel therapies for cancer and David Vetrie will present his recent work on using DNA microarray in improvement of health. We are particularly pleased to welcome Peter Lichter who is well known as a pioneer of molecular cytogenetics. In recognition of his contribution to the field, we have given the all delegates the opportunity to hear his presentation by starting the new methodologies session half an hour early at 2 p.m. Peter has selected a number of leaders in devising new technology for our field and so we look forward to the contributions in this session from Guido Sauter on tissue specific DNA microarrays, Roland Eils discussing elaborate methods for visualizing and analysing
3D genome organization, and Andreas Ladurner: with the intriguingly titled "ChIP on Chip" presentation. This is in addition, to the session from the selected abstracts on Monday afternoon.

Another newly introduced session will be one on population cytogenetics, chaired by Jeremy Searle. Jeremy will enlighten us on the use of chromosomal variation as a marker in natural populations. Johannes Vogel will present his work on identifying areas where plants have survived glaciers using chromosome studies of the fern Asplenium. Natural selection is studied by Antonio Fontdevila by assessing the role of the temperature in latitudinal chromosomal clines in Drosophila. Ira Greenbaum is presenting data on studies relating to the identification, variation and evolution of common fragile sites in human and deer mice. Dave Rowell travels here to speak to us about the causes and consequences of Robertsonian translocations using different organisms that have repeated Robertsonian translocation occurrence or are stable for this event. Kevin Livingstone will discuss his theories on the role of chromosome rearrangements in speciation. Juan Camacho will inform us about supernumerary chromosomal elements in population dynamics.

An all-day session on meiosis and aneuploidy will also be a feature of Thursday with Terry Hassold and Pat Hunt teaming up to head the foremost proponents of the fields. Christer Höög, Sharon Bickel, Stephanie Sherman, Wendy Robinson, Kim Nasmyth, Paula Cohen, Rolff Jessberger and Antoine Peters will cover areas such as age-related non-disjunction in oocytes, meiotic-specific proteins, the role of recombination in aneuploidy, meiosis in fetal oocytes, and synaptonemal complex length. Meiotic errors in the form of aneuploidy are amongst the most common genetic abnormalities known to mankind leading to pregnancy loss, stillbirth, birth defects and mental retardation. Despite this we know relatively little about their causes and thus are some way from effective treatments. We look forward to hearing about the latest research in this most crucial of areas.

Cytogenetics has done tremendous amounts for the cancer biology field in terms of diagnosis and cause. Thus we have two sessions on tumour cytogenetics with a number of world-leaders speaking about how they use chromosome biology in their 
cancer research. Tumour cytogenetics is divided into two sessions covering solid tumours and haemocytological malignancy. Speakers in the solid tumour session will present data on automation to detect malignancy associated changes (Calum MacAulay), advances in microarray technology combined with comparative genome hybridization (Donna Albertson), breast cancer (Jorma Isola), premature condensation of chromosomes to aid in chromosome analysis in slow growing tumours (Karoly Szuhai) and assessing genomic imbalances in neuroblastoma employing a variety of techniques. Hans Tanke, the chair of this session, will give an overview on modern day technologies used to study the genome in solid tumours. In the haemotological malignancy session, chaired by Martin Bentz, an expert in lymphoprolific disorders, the diseases that will be covered include mantle cell lymphoma (Martin Dyer), acute lymphoblastic leukaemia and non-random aneuploidy associated with it (Oskar Haas) and adult acute myeloid leukaemia and gene expression profiles that allow sub-grouping of patients (Jonathan Pollack).

We are particularly delighted to be able to present our conference dinner which will take the form of an old English Medieval banquet complete with traditional music and entertainment from the renowned Dragonsfire (http://www. dragonsfire.btinternet.co.uk/). This will be followed by traditional medieval dancing also led by Dragonsfire. Bring an empty stomach with you and leave your table manners behind!

\section{Day 5}

The morning of our final day (Friday 10 September) will see the whole conference reunited for prize giving and late-breaking news. Nominees for the prizes were chosen by the local organizing committee from those abstracts who made the early submission deadline and contributed a manuscript to a special issue of Cytogenetic and Genome Research. They include multicolour meiotic preps of Codina-Pascual and colleagues, atomic force microscopy studies of Takeyasu et al. in prokaryotes and Hoshi et al. in human chromosomes. Silahtaroroglu et al. will demonstrate the use of Locked Nucleic Acid
(LNA) probes for FISH experimentation in place of DNA or PNA probes, Uchiyama et al. will show the use of $2 \mathrm{D}$ electrophoresis to analyse the protein composition of human chromosomes, Laner et al. will demonstrate the use of a EGFP in transfection efficiencies of human artificial chromosome generated from PAC constructs containing artificial centromeres and telomeres and Bailey et al. present their work on the applications of strand-specific CO-FISH. Lightfoot and Höög present a study of embryos from a model "Scyp-3-null" mouse that produces aneuploid oocytes and subsequent embryos after fertilisation with normal sperm. Rotkov et al. will present an analysis of telomeres in Asparagales while Herron et al,, in studying grasshopper B chromosomes, propose that they persist in evolution via a metaphase checkpoint alteration that, in turn, was more forgiving of misaligned chromosomes. The evolutionary theme is continued by Ferreri et al. with a study of the centromere in marsupials drawing general conclusions about the role of this vital structure in chromosome evolution. We look forward to hearing all their presentations on the final day of the conference and to the choosing of the prize-winners by the International Advisory Panel. Finally, we invite all our delegates to consider their most recent research for our late-breaking news session. The purpose of this session is to keep delegates as up-to-date as possible with the most recent developments in the field of chromosome research.

So, in conclusion, at Brunel we are gathering some of the world's best scientists to celebrate the successes of chromosome research and look to its future achievements. We plan outstanding scientific and social programmes and even a plenary session on chromosome engineering. Isambard Kingdom Brunel, we are sure, would have liked to attend!

\author{
Joanna M. Bridger and Darren K. Griffin \\ Cell and Chromosome Biology Group \\ Dept of Biological Sciences \\ Brunel University \\ West London \\ UB8 $3 P H$ \\ $\mathrm{UK}$
}




\section{Conference Program}

\begin{tabular}{|c|c|c|}
\hline & Sunday 5th September & Monday 6th September \\
\hline $7: 30$ & & \\
\hline 8:00 & & Conference hall opens \\
\hline $8: 30$ & & Start of Conference \\
\hline $9: 00$ & & Keynote address: \\
\hline $9: 30$ & & Prof. John Burn - "The Future of Genetics" \\
\hline 10:00 & & Break \\
\hline 10:30 & & Plenary Session 1: Chair - Dr Darren Griffin \\
\hline 11:00 & & The 3 eras of chromosome research \\
\hline $11: 30$ & & Prof. Pat Jacobs, Dr Adrian Sumner, Prof. David Ward \\
\hline $12: 00$ & Registration desk opens & Lunch \\
\hline $12: 30$ & & Start of Poster Session 1 \\
\hline 13:00 & & \\
\hline 13:30 & & \\
\hline $14: 00$ & & \\
\hline $14: 30$ & & Plenary Session 2: Chair Dr Joanna Bridger \\
\hline $15: 00$ & Meeting of Int. Advisory Committee & Chromosome Engineering \\
\hline $15: 30$ & & Prof. Hunt Willard, Dr Christine Farr \\
\hline 16:00 & & Break \\
\hline $16: 30$ & & Prof. Andy Choo \\
\hline $17: 00$ & & Poster viewing \\
\hline $17: 30$ & & \\
\hline 18:00 & & \\
\hline 18:30 & & \\
\hline 19:00 & Welcome reception & Chromosome Research reception \\
\hline $19: 30$ & & \\
\hline 20:00 & & $\begin{array}{l}\text { FISH and chip supper, real ale, trad jazz } \\
\text { Film shows }\end{array}$ \\
\hline
\end{tabular}




\begin{tabular}{|c|c|c|c|}
\hline & Tuesday 7th September & \multicolumn{2}{|c|}{ Wednesday 8th September } \\
\hline $7: 30$ & Mentor breakfasts & \multicolumn{2}{|l|}{ Mentor breakfasts } \\
\hline 8:00 & & & \\
\hline $8: 30$ & & & \\
\hline $9: 00$ & Morning concurrent sessions start: & \multicolumn{2}{|c|}{ Morning concurrent sessions start: } \\
\hline $9: 30$ & Reproduction, Animal Chromosomes, Centromeres, & \multicolumn{2}{|c|}{ Sex Chromosomes, Plant Chromosomes, Prokaryotes, } \\
\hline $10: 00$ & Genome Organisation & \multicolumn{2}{|c|}{ Chromosome Transfer, Epigenetics } \\
\hline \multicolumn{4}{|l|}{$10: 30$} \\
\hline \multicolumn{4}{|l|}{$11: 00$} \\
\hline $11: 30$ & Dining room opens for lunch & \multicolumn{2}{|c|}{ Dining room opens for lunch } \\
\hline $12: 00$ & & \multicolumn{2}{|c|}{ Start of Poster Session 2} \\
\hline \multicolumn{4}{|l|}{$12: 30$} \\
\hline \multicolumn{4}{|l|}{ 13:00 } \\
\hline $13: 30$ & & Trip A: & Trip B: \\
\hline $14: 00$ & & Virginia Water & Tate Modern \\
\hline $14: 30$ & Afternoon concurrent sessions start & and Windsor & London Eye \\
\hline $15: 00$ & Genome Organisation (cont), Comparative Genomics, & & and West End \\
\hline $15: 30$ & Centromeres (cont.), Plant Chromosomes, Telomeres, & & \\
\hline $16: 00$ & New methodologies & & \\
\hline \multicolumn{4}{|l|}{$16: 30$} \\
\hline 17:00 & Poster viewing & & \\
\hline \multicolumn{4}{|l|}{$17: 30$} \\
\hline \multicolumn{4}{|l|}{ 18:00 } \\
\hline \multicolumn{4}{|l|}{$18: 30$} \\
\hline \multicolumn{4}{|l|}{ 19:00 } \\
\hline \multicolumn{4}{|l|}{ 19:30 } \\
\hline 20:00 & $\begin{array}{l}\text { Classical music recital } \\
\text { Film shows }\end{array}$ & Film shows & \\
\hline
\end{tabular}




\begin{tabular}{|c|c|c|}
\hline & Thursday 9th September & Friday 10th September \\
\hline $7: 30$ & Mentor breakfasts & Mentor breakfasts \\
\hline $8: 00$ & & \\
\hline $8: 30$ & & \\
\hline 9:00 & Morning concurrent sessions start: & Presentations by prize nominees \\
\hline $9: 30$ & Post-Genomic Era, Meiosis, Population Cytogenetics, & \\
\hline $10: 00$ & Tumour Cytogenetics (solid), Gene Therapy & Break \\
\hline $10: 30$ & & \\
\hline $11: 00$ & & \\
\hline $11: 30$ & Dining room opens for lunch & \\
\hline $12: 00$ & & Lunch \\
\hline $12: 30$ & & \\
\hline 13:00 & & \\
\hline $13: 30$ & & \\
\hline $14: 00$ & & Late breaking news session \\
\hline $14: 30$ & Afternoon concurrent sessions start & \\
\hline $15: 00$ & New methodologies, Aneuploidy, Sex Chromosomes (cont.) & \\
\hline $15: 30$ & Tumour Cytogenetics (haematological) & Closing remarks, prize giving \\
\hline $16: 00$ & & End of conference \\
\hline $16: 30$ & & \\
\hline $17: 00$ & Poster viewing & \\
\hline $17: 30$ & & \\
\hline $18: 00$ & & \\
\hline $18: 30$ & & \\
\hline 19:00 & & \\
\hline $19: 30$ & & \\
\hline $20: 00$ & $\begin{array}{l}\text { Conference dinner: } \\
\text { Medieval banquet with traditional entertainment and dancing }\end{array}$ & \\
\hline
\end{tabular}





\section{Plenary Session}

\section{L01}

\section{The future of genetic research}

\section{John Burn}

Institute of Human Genetics, International Centre for Life, Central Parkway, Newcastle upon Tyne, NE1 3BZ, UK

Keynote talk for the conference.

\section{L02}

\section{The classical era}

\section{Pat Jacobs ${ }^{1}$}

${ }^{1}$ Wessex Regional Genetics Laboratory, Salisbury District Hospital, Salisbury, SP2 8BJ and Division of Human Genetics, University of Southampton School of Medicine, Southampton, SO16 6YD

The classical era of human cytogenetics dates from the publication by Tjio and Levan in 1956 of the correct chromosome number of our species. Their observation was confirmed by Ford and Hamerton and it is from that time that the resurgence of human cytogenetics and the contribution of chromosome abnormalities to disease can be dated.

The main autosomal trisomies, the numerical abnormalities of the sex chromosomes and both balanced and unbalanced structural rearrangements of the chromosomes were all discovered without the benefit of banding techniques. Furthermore the amazingly high frequency of chromosome abnormalities among spontaneous abortions was described and showed just how common chromosome abnormalities are in our species. The first recognition of an acquired abnormality, the Philadelphia chromosome, ushered in a new approach to the study and understanding of malignancies, in which chromosome status not only defined the disease, but also the type of treatment and its efficacy. It was a chromosome abnormality, the fragile $\mathrm{X}$, which gave the first clue to a whole new class of mutations, namely trinucleotide repeat expansions, which have proved so important in our understanding a wide range of neurological diseases.

The advances made in the 15 years of the classical era are indeed astonishing. Research undertaken in an attempt to understand the causes of some of the abnormalities recognised during this period and the way this has influenced our thinking about human genetics will be described.

\section{L03}

\section{Chromosomes - the banding era}

Adrian Sumner ${ }^{1}$

${ }^{1} 7$ Smileyknowes Court, North Berwick EH39 4RG, UK

Chromosome banding - the differential staining of parts of chromosomes - began no later than the 1890 s, only some 20 years after the study of chromosomes began, and is today still extensively used. Thus there is really no distinctive banding era: since the early days cytogeneticists have tried to differentiate different regions of chromosomes, and today banding is an essential part of the study of chromosomes, to which all other observations refer. Nevertheless, banding is often held to have started in 1968 when Caspersson and his colleagues first stained chromosomes with quinacrine mustard and obtained longitudinal differentiation of plant and mammalian chromosomes. Since that date a wide variety of techniques have been developed for studying heterochromatic and euchromatic banding, the centromeres, and the nucleolus organisers. The important difference between pre- and post-1968 banding techniques is that the latter are of general application and can be applied to routine chromosome preparations, and are applicable to all organisms with little modification.

Chromosome banding is now mature technology, used routinely for chromosome identification, as a reference for localising FISH and immunofluorescence signals on chromosomes, for evolutionary studies, and for studying chromosomal changes in cancer. Curiously, in spite of much effort, the biological basis of chromosome banding is still poorly understood, as is its phylogenetic distribution. While chromosome banding will continue to be a vital practical tool for the 
foreseeable future, investigations of its structural and functional significance will be important for our understanding of chromosome organisation.

\section{L04}

\section{Plenary Talk - The FISH era}

\section{David Ward}

Molecular Biophysics and Biochemistry Department, Yale University, 333 Cedar Street, New Haven, CT 06520, USA

No abstract was submitted for this talk.

\section{L05}

Chromonomics: The human genome from the chromosome's point of view

\section{Huntington Willard}

Department of Molecular Genetics and Microbiology, Duke University, Box 3382, Durham, North Carolina 27710, USA

No abstract was submitted for this talk

\section{L06}

\section{Chromosome engineering: its} application in vector development and in the functional dissection of vertebrate centromeres

\section{Christine Farr ${ }^{1}$}

${ }^{1}$ Department of Genetics, University of Cambridge, Downing St, Cambridge CB2 3EH UK

We have used the targeted manipulation of a human chromosome, maintained in a somatic cell hybrid, to generate an extensive series of linear minichromosomes. These are being used to (i) investigate the requirements of a chromosomebased vector system for vertebrate cells; and (ii) to functionally dissect the centromere domain. Our progress in both these areas will be discussed.
L07

What price neocentromere?

\author{
Andy Choo ${ }^{1}$ \\ ${ }^{1}$ Murdoch Childrens Research Institute, \\ Melbourne, Australia
}

Neocentromeres are fully functional centromeres that are formed ectopically at non-centromeric locations of the genome. During neocentromere formation, unknown epigenetic mechanisms underlie the transformation of non-centromeric, often gene-encoding genomic regions into centromeric chromatin. The principal trigger for neocentromere formation is through chromosomal rearrangements that result in the loss of the endogenous functional centromere on a chromosome or chromosomal fragment. In humans such chromosomal rearrangements often cause karyotype imbalance that leads to genetic abnormalities and even cancer. Given the general detrimental effects of neocentromere formation to the individual, what is the biological advantage of having a neocentromere forming mechanism? One model is to provide a potentially powerful driving force for karyotype evolution and speciation through a process of centromere repositioning. We discuss recent evidence in humans, lower primates, and plant in support of such a model.

Fully sequenced human neocentromeres also provide unique practical advantages as a tool for the dissection of the complex properties of the mammalian centromeres. Use of neocentromeres has allowed us to define the extent of enhanced chromosomal scaffold/matrix binding at the human centromere and to show the permissibility of transcription within the enhanced scaffold/ matrix-binding domain. It has also allowed us to begin the construction of a linear organisational map showing distinct and non-overlapping centromere protein-binding domains, and the malleability of these domains in response to chromosomal truncation or treatment with histone or chromosomal scaffold modification agents. 


\section{Animal Chromosomes Symposium}

L09

Molecular cytogenetic definition of the chicken genome: The first complete avian karyotype

D. Griffin ${ }^{1}$, J. Masabanda ${ }^{1}$, D. Burt ${ }^{2}$,

P. O'Brien ${ }^{3}$, M. Groenen ${ }^{4}$, A. Vignal ${ }^{5}$,

R. Crooijmans ${ }^{4}$, V. Fillon ${ }^{5}$,

M. Ferguson-Smith ${ }^{3}$ and J. Smith ${ }^{2}$

${ }^{1}$ Cell and Chromosome Biology Group, Brunel University, UK; ${ }^{2}$ Roslin Institute, UK;

${ }^{3}$ University of Cambridge, UK; ${ }^{4}$ Waageningen

University, Germany; ${ }^{5}$ INRA, Toulouse, France

Chicken genome mapping is important for a range of scientific disciplines and the ability to distinguish chromosomes of chicken and other birds is thus a priority. Here we describe the molecular cytogenetic characterisation of every chicken chromosome using chromosome painting and mapping of individual clones by fluorescence in-situ hybridization FISH. Where possible we have assigned the chromosomes to known linkage groups. We propose, on the basis of size, that the NOR chromosome is around the size of chromosome 22; however we suggest its original assignment of 16 should be retained to avoid confusion. We suggest the definitive chromosome classification system and propose that the probes developed here will find wide utility in the fields of developmental biology, DT40 studies, agriculture, molecular ecology, vertebrate genome organisation and comparative mapping of avian species.

\section{L10}

Use of FISH-mapped genes to construct an integrated genetic map in rabbit

H. Hayes ${ }^{1}$, C. Chantry-Darmon ${ }^{2}$, D. Allain ${ }^{3}$, B. Pena ${ }^{3}$, C. Urien ${ }^{2}$, M. Bertaud ${ }^{1}$, H. De Rochambeau ${ }^{3}$ and C. Rogel-Gaillard

${ }^{1}$ Laboratoire de Genetique biochimique et Cytogenetique, INRA, 78352 Jouy-en-Josas Cedex, France; ${ }^{2}$ Laboratoire de Radiobiologie et Etude du Génome, UMR INRA CEA, 78352 Jouy-en-Josas Cedex, France; ${ }^{3}$ Station d'Amelioration Genetique des Animaux, INRA, BP27, 31326 Castanet-Tolosan Cedex, France

Rabbits are bred both for the production of fur and meat and for biomedical research. It is still a map-poor species and thus our long-term objective is to provide mapping data and tools, which will help increase the likelihood of finding genes associated with complex multifactorial traits. Since mapping data is very limited in rabbit, we have decided to construct an integrated genetic and cytogenetic map using a direct strategy. First, we have chosen 350 genes regularly distributed over the human genome approximately every $10 \mathrm{cM}$, hypothesizing that they will be equally regularly distributed in rabbit. A threegenome equivalent rabbit BAC library has been screened for each of these genes and we isolated clones for 302 genes. On the one hand, these clones are being localized on rabbit chromosomes by fluorescent in situ hybridization (FISH) and on the other hand, they are being used to isolate microsatellite markers. Eight reference rabbit families will be genotyped with these markers to construct a genetic map. Thus, the original feature of this strategy is the direct construction of an integrated map, the cytogenetic results providing accurate comparative mapping data with man. Indeed, in bovine and porcine species, cytogenetic and genetic maps were built separately and then integrated. To date, 180 gene-containing BAC clones have been FISH-mapped, which represents a four-fold enrichment of the rabbit gene map and 161 microsatellite markers have been isolated. We shall present an updated version of the cytogenetic gene map and the first integrated genetic map in rabbit.

\section{L11}

\section{Genome and nuclear architecture} organisation during development and differentiation using the pig as a model organism

H. Foster ${ }^{1}$, R. Sturmey ${ }^{2}$, P. Stokes ${ }^{2}$, H. Leese ${ }^{2}$, L. Abeydeera ${ }^{3}$, D. Griffin ${ }^{1}$ and J. Bridger ${ }^{1}$ 
${ }^{1}$ Cell and Chromosome Biology Group, Biological Sciences Department, Brunel University, Uxbridge, Middlesex, UB8 3PH, UK.; ${ }^{2}$ Department of Biology, The University of York, PO Box 373, York, YO10 5YW, UK.; ${ }^{3}$ Sygen International, P. O. Box 348, 3033 Nashville Road, Franklin, KY 42135, USA.

The precise organisation of the genome and its environment, the cell nucleus, is critical for correct development and differentiation. Thus, we are performing studies to analyse genome organisation and nuclear architecture at important stages in early development and differentiation.

We have analysed the nuclear position of the majority of porcine chromosomes in a number of in vitro cultured cell-types: embryonic fibroblasts, adult fibroblasts, lymphocytes and mesenchymal stem cells. Apart from one chromosome all chromosomes are positioned in similar places in the nucleus.

To study nuclear and genome organisation in differentiated cells we have established in vitro differentiation systems as models for cell differentiation during embryogenesis by differentiating porcine mesenchymal stem cells into myocytes and adipocytes. To compare in vitro with in vivo growth conditions we are studying the same aspects of nuclear and genome organisation in frozen tissue sections.

For developmental studies we have assessed chromosome positioning in sperm from three different breeds of pig. These chromosomes are nonrandomly organised both radially and longitudinally. Chromosome positioning in sperm is also totally different to somatic cells and thus, may have a role in controlling early paternal gene expression in the new embryo.

We are also comparing aspects of genome and nuclear organisation in in vivo and in vitro constructed porcine embryos. This will reveal any detrimental alterations common in IVF embryos and may lead to improved selection criteria for IVF embryos.

These studies presently make the pig the organism that has the most chromosomes mapped in the most cell types.
L12

\section{Geographical analysis of a chromosomal hybrid zone in the common shrew}

\author{
R. Jones ${ }^{1}$ \\ ${ }^{1}$ Department of Biology, University of York, \\ PO Box 373, York YO10 5YW, UK
}

The common shrew (Sorex araneus) is subdivided into numerous races distinguished by Robertsonian fusions and whole-arm reciprocal translocations. Two of these races, the Oxford and Hermitage, make contact in Britain and form a zone of hybridisation. Hybrid zones are of interest because they may be sites of speciation, i.e. where gene flow is halted. In fact the OxfordHermitage hybrid zone has a structure that is unlikely to promote speciation; instead natural selection has led to a structure where gene flow is maximised. The zone was characterised along a small part of its length in the 1980s. I have now mapped nearly the whole length of the zone between the Thames, Severn and Mersey estuaries and the structure is the same along this total length, consistent with powerful selective forces favouring that structure. The detailed mapping of such a long hybrid zone provides a valuable opportunity to relate hybrid zone position and width to geographic features and I will present an analysis of this in which Geographic Information Systems and population genetic mapping techniques have been applied.

\section{L13}

Avian lampbrush chromosomes: molecular composition and variety of lateral loops and chromosome associated structures

E. Gaginskaya ${ }^{1}$, S. Derjusheva ${ }^{1}$, A. Krasikova ${ }^{1}$, T. Kulikova ${ }^{1}$, A. Kurganova and A. Saifitdinova ${ }^{1}$

${ }^{1}$ Biological Research Institute of Saint-Petersburg University, 198504, Russia 
Meiotic diplotene chromosomes in germinal vesicles $(\mathrm{GVs})$ of avian oocytes transform to typical lampbrush chromosomes (LBCs). Numerous simple lateral loops are characterized by intensive transcription and rather uniform morphology when stained with antibodies against phosphorylated form of RNA polymerase II (pol-II) or probed with chromosome paints according to DNA/RNA hybridization protocol. The variety of simple loops clarifies itself under immunocytochemical investigation of bound proteins. Lateral loops, specifically enriched with either mRNA processing protein $\mathrm{K}$, or dsRNA-editing enzyme ADAR, or a protein sharing the epitope with RPC39 subunit of RNA pol-III, etc., have been revealed. This intriguing feature appears to be a general functional characteristic of pol-II lateral loops on LBCs. Earlier, such a peculiarity of individual lateral loops was also described for amphibian LBCs. Lateral loops of complex morphology, in particular telomeric giant loops (TGLs) on avian LBCs, appear not to be transcribed by pol-II. TGLs accumulate splicing snRNPs and SR protein SC35 being considered as a kind of SC35 domains in avian GVs. Chromosome associated structures of various morphology (protein bodies, spaghetti marker and some others) do not either reveal any transcriptional activity and are mainly composed of proteins of special nature. The protein bodies may reflect a specific function of centromeric DNA repeats. An attempt to systematize the types of lateral loops according to their molecular composition and suggested function has been made.

The research is supported by Russian Foundation for Basic Research (project 02-04-49116), and partly by Russian Ministry of Education and Science (projects PD02-1.4-291 and 2655.2003.04).

\section{L14}

\section{Changes in chromosomal surface structure are directly related to protein composition}

\author{
K. Fukui ${ }^{1}$, S. Uchiyama ${ }^{1}$, T. Sone ${ }^{2}$, M. Iwano ${ }^{3}$ \\ and S. Matsunaga ${ }^{1}$
}

${ }^{1}$ Department of Biotechnology, Graduate School of Engineering, Osaka University; ${ }^{2}$ Institute for Microbiological Diseases, Osaka University; ${ }^{3}$ Nara Advanced Institute of Science and Technology, Japan

Despite more than a century of research on chromosome structure, it still remains poorly understood. One of the reasons for this situation has been the difficulty in the biochemical study of metaphase chromosomes.

In the present study, isolated human metaphase chromosomes, which were prepared from the synchronized human cell line by a centrifugation method by two representative solutions for chromosome isolation (polyamine buffer, PAB and citric acid solution, CAS), were examined by both scanning electron microscopy (SEM) and one-dimensional SDS-PAGE. SEM observation of isolated chromosomes in PAB and CAS revealed the two types of surface structures which have repeatedly been reported to date. The surface of the human chromosomes in PAB was relatively flat structure covered irregularly with scales different in shapes and sizes, while the surface of the chromosome in CAS had the dense fibrous structure with uniform diameter of 50-70 nm. Analyses of protein compositions of these two chromosomes by one-dimensional SDS-PAGE showed the marked difference from each other where linker histone, H1, was removed from the chromosomes isolated with CAS. These results suggested that the removal of chromosomal proteins including the linker histone might be responsible for the specific morphology of the chromosome surface structure. Identification of chromosomal proteins by biochemical analysis combined with the morphological analysis would provide the essential data for the elucidation of higher order structure of metaphase chromosomes.

\section{L15}

\section{Differential gene expression in} yellow-necked mice Apodemusflavicollis with and without $B$ chromosomes

\section{N. Tanic ${ }^{1}$, M. Vujosevic ${ }^{1}$, N. Dedovic-tanic ${ }^{2}$ and B. Dimitrijevic ${ }^{2}$}

${ }^{1}$ Institute for Biological Research 'Sinisa Stankovic' ${ }^{2}$ Institute for Nuclear Sciences 'Vinca' 
Most B chromosomes are heavily heterochromatic promoting the general idea that they are genetically inert. B chromosomes of Apodemus flavicollis are euchromatic and show high degree of homology with the A chromosomes. Euchromatic nature of Bs in A. flavicollis suggests that they may carry active genes and have transcriptional activity. We applied Differential Display Reverse Transcription - Polymerase Chain Reaction (DD RT-PCR) in order to analyze and compare gene expression in animals possessing Bs and animals without B chromosomes. After second and third round of amplification three cDNA fragments were differentially expressed in $+\mathrm{B}$ mice compared to $0 \mathrm{~B}$ animals. These cDNAs were: chaperonin containing TCP-1, subunit 6b (zeta) (Cct6b), Fragile histidine triad gene (Fhit) and hypothetical gene XP transcript. Differential expression pattern was confirmed by Real Time RT-PCR. Nature of Bs and significance of these effects are discussed in the context of presented data.

\section{L16}

\section{BAC-FISH karyotyping in Bombyx mori}

\author{
K. Sahara ${ }^{1}$, A. Yoshido ${ }^{1}$ and Y. Yasukochi ${ }^{2}$ \\ ${ }^{1}$ Graduate School of Agriculture, Hokkaido \\ University, Japan; ${ }^{2}$ Genome Research \\ Department, National Institute of Agrobiological \\ Sciences, Japan
}

Chromosome identification is fundamentally important for detection of gene mutations such as point mutations, insertions, deletions, inversions or translocations. Cytogentic mapping and the generation of BAC libraries are inevitable requirements of whole genome shotgun sequencing projects.

In Lepidoptera, chromosomes are generally small, and uniform in shape and size without primary constrictions in mitotic metaphase. Since an applicable banding technique has not yet been found, chromosome identification and cytogenetic mapping were thought to be not feasible. The silkworm, Bombyx mori $(2 \mathrm{n}=56$ chromosomes), has been one of the most problematic lepidopteran species to be employed in chromosome research.
On the other hand, the progress of scientific studies on the molecular level including the genome project requires chromosome identification. The success of FISH using $B$. mori BAC probes (Sahara et al. 2003) opens the gate of BAC-FISH chromosome identification. A library consisting of 36,864 BACs constructed from two strains (Wu et al., 1999) and, physical map based on RAPDs and mutants (Yasukochi 1998; Fujii et al. 1998) are the basis of this approach.

Here we identify all 28-chromosome bivalents including the WZ by using BAC-FISH in B. mori. Positions of hybridization signals from BAC probes correspond roughly to physical map position. Moreover, we accomplish B. mori karyotyping using 2-color combination of $60 \mathrm{BAC}$ probes. We expect the BAC-FISH method is universally applicable for karyotyping of species in which chromosome identification is problematic.

\section{L17}

\section{A tandemly repetitive centromeric DNA sequence from the fish Hoplias malabaricus is derived from the $5 \mathrm{~S}$ rDNA}

\author{
C. Martins ${ }^{1}$, I. Ferreira ${ }^{1}$, C. Oliveira ${ }^{1}$, \\ F. Foresti ${ }^{1}$ and P. Galetti $\mathrm{Jr}^{2}{ }^{2}$
}

${ }^{1}$ Departamento de Morfologia, Instituto de Biociencias, Universidade Estadual Paulista, CEP 18618-000, Botucatu, SP, Brazil. ${ }^{2}$ Departamento de Genética e Evolucio, Universidade Federal de Sao Carlos, CEP 13565-905 Sao Carlos, SP, Brazil

A substantial fraction of the eukaryotic genome, in some instances greater than $90 \%$, consists of repetitive DNA sequences that include satellite, minisatellite, microsatellite, and transposable elements. Although extensively studied for the past four decades, the molecular forces that propagate and maintain repetitive DNAs in the genome are still not well understood. To further the understanding on the dynamics and evolution of repetitive DNAs in vertebrate genome, we have searched repetitive sequences in the genome of the fish Hoplias malabaricus, a model species for genetic studies. We have discovered a tandemly repetitive DNA family, named Hmall, that has 
derived from repeat units of the $5 \mathrm{~S}$ rDNA by insertions/deletions and base substitutions. The 5S rRNA bona fide gene repeats are clustered in the interstitial position of two chromosome pairs and its $5 \mathrm{~S}$ variant type is clustered in the centromeric region of nine chromosome pairs. The satellite sequence Hmall could has arisen by transposition from the bona fide $5 \mathrm{~S}$ gene repeats and spread out in the centromeric region of several chromosomes due to the action of concerted evolution events. Such results are evidencing that the Hmall family escaped from the selective pressure that maintain the structure and organization of the 5S rDNA and is free to disperse in the genome and has been maintained in the centromeric regions because of any structural advantage conferred by a possible centromeric function in the chromosomes.

\section{PO:06:01}

\section{New insights into Bovidae satellite III evolutionary history}

F. Adega', R. Chaves ${ }^{1}$, J. Heslop-Harrison ${ }^{2}$, J. Wienberg ${ }^{3}$ and $H$. Guedes-Pinto ${ }^{1}$

${ }^{1}$ Department of Genetics and Biotechnology, Centre of Genetics and Biotechnology, Vila Real, Portugal; ${ }^{2}$ Department of Biology, University of Leicester, UK; ${ }^{3}$ Institute of Human Genetics, University of Munich, Munich, Germany

Repeats like satellite DNA sequences constitutes a rich palaeontological record, holding crucial clues about evolutionary events. As in the others mammalian orders, the Bovidae centromere is greatly composed of satellite DNA families. Satellite 1.706 (satIII) is a long unit of $2350 \mathrm{bp}$ composed of complex and altered repetitions of two related and homogeneous 23-mer tandem subrepeats, the Pvu and the Sau motifs.

The scope of this work was the isolation, sequencing and analysis of the genome organization and physical distribution (by Southern Blot and Fluorescent in situ Hybridization) of these satellite sequences in several Bovidae species.

The procedures used showed that the presence of this satellite family was not restricted to the Bovini (Bos taurus, Bos indicus and Bubalus bubalis), as described in previous works; instead we detected satellite III on the Tragelaphini (Tragelaphus euryceros). This finding could be revealing of an earlier appearance or formation of 1.706 satellite III DNA, prior to the Bovini and Tragelaphini divergence.

Although the existence of satellite III in water buffalo (Bubalus bubalis) had been reported, it was noticed to only one autosomal pair. In the present work we were able to observe signal hybridisation in the majority of the autosomes, and remarkably, in the sex chromosome $\mathrm{X}$, in contrast with the absence of satIII in Bos chromosome X. These observations are revealing of a different evolutionary mechanism for the sex chromosome $\mathrm{X}$ of this species.

\section{PO:06:02}

\section{Karyological comparative study of the genus Leiolepis (Reptilia, Uromastycidae) from Thailand}

\author{
V. Aranyavalai ${ }^{1}$, W. Chulalaksananukul ${ }^{2}$ and \\ K. Thirakhupt ${ }^{3}$
}

${ }^{1}$ Biological Science Program, Faculty of Science, Chulalongkorn University, Bangkok 1033, Thailand; ${ }^{2}$ Genetic Program, Department of Botany, Faculty of Science, Chulalongkorn University. Bangkok 10330, Thailand; ${ }^{3}$ Department of Biology, Faculty of Science, Chulalongkorn University, Bangkok 10330, Thailand

The karyotype of four species of butterfly lizards collected in Thailand, Leiolepis belliana belliana, L. belliana ocellata, $L$. reevesii rubritaeniata, were described in detailed for the first time. All have $2 \mathrm{n}=36$ chromosomes and the same karyotypic formulae were $10 \mathrm{~m}+2 \mathrm{sm}+24 \mathrm{mc}$ (12 macrochromosomes and 24 microchromosomes). The L. boehmei has $2 \mathrm{n}=34$ chromosomes and its karyotypic formula was $10 \mathrm{~m}+2 \mathrm{sm}+22 \mathrm{mc}(12 \mathrm{mac}-$ rochromosomes and 22 microchromosomes). The higher microchromosome number occurred in the first three types of lizard which may be due to the karyotypic change by centric fission in $L$. boehmei. All the time of our exploration, L. boehmei male was never found in southern part of Thailand, indicating that this population was a unisexual species of Leiolepis. Moreover, the 
difference in microchromosome number of $L$. boehmei compared to the others can be explained more clearly by Von Wolfgang Bähme (1982) about the Leiolepis triploida found in south of Thailand. It was hypothesized that triploid could be allotriploid hybrid clone between the diploidparthenogenetic female and males of the normal bisexual species. The hypothesis of $L$. triploida occurrence can be proposed from our karyotypic data. The relationship of these species with the other congeneric species will be described from the cytotaxonomic point of view.

\section{Reference}

Bähme, W. 1982. Uber schmetterlingsagmen, Leiolepis $b$. belliana (Gray, 1827) der Malayischen halbinsel und ihre parthenogenetischen linien (Sauria: Uromastycidae). Zool. Jb. Syst. 109: 157-169.

\section{PO:06:03}

\section{Hypercondensation; an old change of conformation that is still somewhat mysterious}

\section{Burgoyne ${ }^{1}$ and D. Repucci ${ }^{1}$ \\ ${ }^{1}$ School of Biological Sciences, Flinders \\ University of South Australia, Bedford Park 5042, Australia}

From the literature, there are four very broad levels of condensation of chromatin in order of increasing condensation., (A) the transcriptionally-enabled (least condensed class), (B) heterochromatic, (C) metaphase-level of condensation, and (D) hypercondensed; pyknotic.the most condensed class. Chromosomes are commonly collapsed into this latter class by colchicine or DNA breakage or other death processes. The process is readily scored by phasecontrast microscopy but it is difficult to study by electron microscopy as handling and or fixatives often induce it before embedding. This presentation is part of an in-vitro study into the nature of pyknosis, its triggers, the events that accompany it, and the causal relationships between the events surrounding it. Evidence will be examined in an attempt to discover any underlying commonality in the processes that trigger hypercondensation and to distinguish two propositions from each other. The first, that the conformational change is due to slight shifts in rotational positioning of adjacent nucleosomes and the second that the conformational change is due to a re-arrangement of the placing of bridge-histones relative to the nucleosome core histones.

\section{PO:06:04}

\section{Cytogenetic characterization of metaphase chromosomes of alpaca (Lama pacos): I-GTG/RBG-banded karyotypes and idiograms}

D. Di berardino ${ }^{1}$, G. Balmus ${ }^{2}$, D. Nicodemo ${ }^{1}$ G. Coppola ${ }^{1}$, C. Verdoliva ${ }^{1}$, G. Coppola ${ }^{1}$, G. Enne ${ }^{3}$, G. Bozzini ${ }^{4}$, G. Di $\mathrm{Meo}^{5}$ and L. Iannuzzi ${ }^{5}$

${ }^{1}$ Department of Animal Science and Food Inspection, University of Naples, Portici, Italy; ${ }^{2}$ Faculty of Veterinary Medicine, Iasi, Romania; ${ }^{3}$ Italian Experimental Institute, Milan, Italy; ${ }^{4}$ Italian Alpaca Breeder Center, Grosseto, Italy; ${ }^{5}$ National Research Council (CNR), ISPAAM, Laboratory of Animal Cytogenetics and Gene Mapping, Naples, Italy

The present study was undertaken to provide GTG- and RBG- banded karyotypes and representative ideograms for the alpaca species (Lama pacos, $2 n=74$ ), to be used as a basis for future work on karyotype standardization and gene mapping, as well as for future genetic improvement programs within the family Camelidae. Peripheral blood cultures from eight alpaca individuals, four males and four females, were set up according to the standard methods. After 48 hours of growth, cells were synchronized with methotrexate; after 16 hours, the S-phase block was released by washing and the cells allowed to grow for further 5-6 hours in fresh medium, supplemented either with thymidine for conventional karyotyping and GTG-banding, or with BrdU/ H33258 for RBG- banding, respectively. The GTG- and RBG- banded karyotypes reveal a large submetacentric X-chromosome, a very small metacentric $\mathrm{Y}$ - and 72 autosomes which 
have been morphologically classified into 2 groups, as follows: group (A) includes 29 pairs, ranging from sub-meta, sub-telo and acrocentrics; group (B) includes 7 distinct pairs of metacentrics. The GTG/RBG-banding pattern of individual chromosome pairs and their ideograms are presented at 362 bands level.

\section{PO:06:05}

\section{Robertsonian translocation in the Caspian minature horse}

\author{
S. Dordari ${ }^{1}$, R. Mehran Nejhad ${ }^{1}$ and \\ S. Seyed Rassaghi ${ }^{2}$ \\ ${ }^{1}$ Tehran Agricultural Research Center, Iran; \\ ${ }^{2}$ Private Veterinary Laboratory, Iran
}

Przewalski's horse has extensive chromosome homology with the domestic horse. Two acrocentric pairs in Equus prezualskii appear to be combined in one metacentric chromosome (chromosome 5) in the E. caballus karyotype. The Caspian horse is one of the exception that has reported as translocation heterozygotes and have not been directly implicated in fertility problems in this breed. This breed of horse has been thought to be a pony type but is one of the miniature horses today. In the recent study on 30 registered Caspian horses, blood samples were used for metaphase chromosomes provided by use of short-term culture and studied by simple staining and Giemsa banding method. The chromosome spreads were photographed and karyotyped. The results showed the diploid model karyotype as $2 n=64$ and $2 n=65$. Those which had 64 chromosomes were obtained 31 autosomal chromosomes that 7 pairs were metacentric and 6 pairs were submetacentric and 18 pairs were telocentric. Preparation with 65 chromosomes were contained similar chromosome type except that one extra metacentric chromosome was included. The $\mathrm{X}$ sex chromose was large metacentric and the $\mathrm{Y}$ chromosome was small telocentric as same as other domestic horse species.

This karyotype with 65 chromosome in the Caspian miniature horse is unique and this extra chromosome is the result of a natural centric fusion obtained from crossing between $E$. przewalski $(2 \mathrm{n}=66)$ chromosomes in which two acrocentric chromosomes were involved in the creation of a metacentric chromosome.

\section{PO:06:06}

The metaphase chromosome proteome

\author{
M. Emrick ${ }^{1}$, K. Resing ${ }^{1}$, L. Aveline-Wolf ${ }^{2}$, \\ K. Meyer-Arendt ${ }^{2}$, K. Pierce ${ }^{1}$ and N. $\mathbf{A h n}^{2}$ \\ ${ }^{1}$ University of Colorado, Boulder, CO; ${ }^{2}$ Howard \\ Hughes Medical Institute
}

Chromosomal condensation and separation of sister chromatids during cell division requires coordinated assembly and function of hundreds of proteins. Malfunction of these proteins, many of which are unknown, can compromise genetic stability, which is critical to cell integrity, proliferation, and survival.

Here, we perform a proteomic analysis of mitotic chromosomes from metaphase-arrested HeLa $\mathrm{S} 3$ cells enriched on a sucrose gradient. Bound proteins are sequentially extracted from the chromosomes with urea then with an acid-labile surfactant (ALS), prior to trypsin proteolysis. The resulting peptides are separated by reverse-phase chromatography before undergoing fragmentation on an LCQ mass spectrometer. The spectra are analyzed using in-house search algorithms that rely on peptide chemical properties, as well as consensus between Sequest and Mascot search programs (Resing et al., 2004).

These preliminary studies identify several known chromatin-binding proteins, including four histones, several transcriptional regulators, many chromosomal structure regulators, and a kinetochore protein. This confirms that our novel extraction procedure enriches for chromosomal proteins. Extraction under these conditions enriched nuclear proteins to $\sim 40 \%$ of the total proteins identified, with an additional $\sim 30 \%$ of unknown localization.

To detect lower abundance proteins, we are improving this procedure with strong cation exchange and gas-phase fractionation. In order to eliminate the identification of contaminating proteins, we plan to employ protein fraction profiling (Anderson et al., 2003). Additionally, immunofluorescence studies will be performed using exogenously expressed proteins to confirm mitotic 
chromosome localization of novel proteins. Studies are also being undertaken to look for post-translational modifications such as acetylation, phosphorylation, and methylation.

\section{PO:06:07}

\section{The role of repetitive DNA sequences for the comprehension of Nile tilapia (Oreochromis niloticus) genome}

\section{Ferreira ${ }^{1}$, R. Shimizu' ${ }^{1}$, C. Oliveira ${ }^{1}$, F. Foresti ${ }^{1}$ and C. Martins ${ }^{1}$}

${ }^{1}$ Departamento de Morfologia, Instituto de Biociencias, Universidade Estadual Paulista, CEP 18618-000, Botucatu, SP, Brazil

The Nile tilapia (Cichlidae, Perciformes) has received increasing scientific interest over the past few decades due to its enormous importance in worldwide aquaculture and the rapid and extensive evolutionary radiation undergone by the cichlid fishes. Data on Nile tilapia genome will immediately accelerate research in basic and applied biology. One unclear point in the genome comprehension is the large regions that contain repetitive sequences. Such regions remain as gaps even in most complete sequenced genomes. Studies of repetitive sequences can provide the understanding of these regions, contributing to a complete physical map for the species. Concerning the Nile tilapia genome, studies on repetitive sequences have been directed to satellite DNAs, telomeres, 45S and 5S rDNAs, and the short and long interspersed nucleotide elements (SINEs and LINEs). Although several repetitive DNA sequences have been characterized, the presence of a large number of repetitive families remains to be described in the genome of Nile tilapia. In this way, we have searched a restriction fingerprint database (freely available at http://hcgs.unh.edu/cichlid/\#bacs.), assembling 35,000 BAC clones (5x genome coverage) from the Nile tilapia. Candidate BACs were identified and dotblot screened for the presence of repetitive sequences. Several BAC clones were isolated and used as probes for the chromosome mapping. These results are contributing to the construction of a physical chromosome map for the Nile tilapia. The physical mapping of repetitive DNAs to metaphase chromosomes represents a powerful toll to the knowledge of the genome of this important teleost fish.

\section{PO:06:08}

\section{Analyses of human chromosomal proteins using monoclonal antibodies}

T. Higashi' ${ }^{1}$, S. Miyakawa ${ }^{1}$, H. Takata ${ }^{1}$, A. Terauchi ${ }^{2}$, T. Shimizu ${ }^{2}$, M. Oda ${ }^{2}$, S. Uchiyama $^{1}$, S. Matsunaga ${ }^{1}$, T. Azuma ${ }^{2}$ and K. Fukui ${ }^{1}$

${ }^{1}$ Department of Biotechnology, Graduate School of Engeneering, Osaka University, Japan;

${ }^{2}$ Division of Biosignaling, Research Institute for Biological Sciences, Tokyo University of Science, Japan

Chromosomes consist of DNA and various kinds of proteins such as histone proteins, high mobility group proteins and topoisomerase II. However, the total image of chromosomal proteins are still remained unclearly. To identify chromosomal proteins and elucidate their nature, we prepared monoclonal antibodies against human chromosomes which were extracted by the polyamine method. After screening of hybridoma strains based on the localization pattern of antigen proteins by the indirect immunofluorescence method, twenty-five strains were detected to produce the monoclonal antibodies against chromosomal or nuclear-localized proteins. Then the antigen-proteins localized at chromosomes or nuclei were studied. The antigen proteins were purified by immunoprecipitation using HeLa cell extracts. The antigen proteins purified, which could be detected by western blotting, were identified by MALDI-TOF MS using the peptide mass finger printing method. Most proteins were core and linker histones. However, nuclear mitotic apparatus protein (NuMA), hnRNP and betaactin were identified as chromosomal proteins by our method. For confirmation of chromosomal localization of these proteins more precisely, the indirect immunofluorescence method using metaphase spreads was also performed. These results indicate that our method is useful for identification of chromosomal and nuclear proteins. 
PO:06:09

\section{SC-FISH of repeated sequences in three species of clams and cockles}

\author{
N. Hurtado ${ }^{1}$, P. Moran ${ }^{1}$ and J. Pasantes ${ }^{1}$ \\ ${ }^{1}$ University of Vigo, Spain
}

The species belonging to the Order Veneroida (Mollusca, Bivalvia) inhabit an extensive variety of marine ecosystems. Although many of these species are commercially important, chromosome research in these organisms is still rather scarce. During the last few years the combination of FISH techniques with surface spreading of whole synaptonemal complexes (SC-FISH) has greatly increased the use of pachytene cells in chromosome research. Nevertheless, none of these methods have been applied to meiotic chromosomes of bivalves.

Synaptonemal complexes were prepared from males of three species of Veneroida, the clams Dosinia exoleta and Spisula solida and the cockle Cerastoderma edule, using a surface spreading technique. FISH was performed using all human telomeric probes and species specific $18+28 \mathrm{~S}$ and $5 \mathrm{~S}$ rDNA probes. Species specific probes for $18+28 \mathrm{~S}$ rDNA internal transcribed spacers (ITS) and 5S rDNA coding and spacer sequences were generated by PCR. The amplification products were analysed by SSCPs, cloned and sequenced.

Major ribosomal gene clusters are localised in only one bivalent in the three species analysed. ITS signals appear in a loose way surrounding the telomeric region of the SC. On the contrary, the telomeric signals appear tightly associated at the end of the SC both on the NOR-bearing region and all the other telomeric regions. The number and position of the $5 \mathrm{~S}$ rDNA clusters is different for each species but they also appear loosely surrounding the SCs.

\section{PO:06:10}

\section{Localization of calreticulin on human metaphase chromosomes}

\author{
S. Kobayashi ${ }^{1}$, A. Kuwae ${ }^{1}$, S. Uchiyama ${ }^{1}$, \\ S. Matsunaga ${ }^{1}$ and K. Fukui ${ }^{1}$ \\ ${ }^{1}$ Department of Biotechnology, Graduate School \\ of Engineering, Osaka University, Japan
}

Calreticulin (CRT) is a calcium binding protein which mainly exists in an endoplasmic reticulum, and functions as a molecular chaperon that assists folding of newly synthesized proteins. On the other hand, recently, the existence of CRT in the nuclear matrix has been reported and its involvement in the regulation of gene expression has been discussed. Investigation of the subcellular localization of CRT through the cell cycle will lead us to understand the function of this multifunctional protein. In this study, specific localization of calreticulin on human metaphase chromosomes was revealed biochemically and immunohistochemically. Quantitative analysis using western-blotting with anti-CRT antibody revealed that the amount of CRT in the metaphase chromosomes was more than three times compared to that in the G2-nuclei. Immunostaining against thin sections of human mitotic cells as well as isolated chromosomes showed the localization of CRT on the surface of the metaphase chromosomes. To identify the binding target of CRT to metaphase chromosomes, we performed the far-western experiment against chromosomal proteins and the binding assay using chromatin fibers purified from metaphase chromosomes. As the result, it was indicated that recombinant human CRT directly binds to core histones. Present results suggest that CRT is one of the proteins transported to daughter cells by mitotic chromosomes during the cell division.

\section{PO:06:11}

\section{Heterochromatin patterns on chromosomes of beetle species (Insecta: Coleoptera). Present stage of knowledge}

\author{
D. Lachowska-Cierlik ${ }^{1}$, M. Rosek ${ }^{1}$, \\ E. Petitpierre ${ }^{2}$ and M. Holecova ${ }^{3}$ \\ ${ }^{1}$ Institute of Systematics and Evolution of \\ Animals Polish Academy of Science, Krakow, \\ Poland; ${ }^{2}$ Laboratori de Genetica, Deparatment de \\ Biologia, Facultat de Ciencies, UIB, Palma de \\ Mallorca, Spain; ${ }^{3}$ Department of Zoology, \\ Comenius University, Bratislava, Slovakia
}

Data on heterochromatin patterns are known only for some of beetle families. It seems that the 
main reason for the small number of data is the limited amount of heterochromatin in the chromosomes of most beetle species. Heterochromatin patterns on the chromosomes of beetle species were studied using the C-banding technique. Banding techniques allow a better characterization of beetle karyotypes and selectively reveal chromosome regions consisting of constitutive heterochromatin. The results confirm that most of the beetle species possess a small amount of heterochromatin. All chromosomes obtained from spermatogonial cells possess C-banded segments which are visible during the pachytene and diplotene. When the chromosomes become more condensed during the mitotic metaphase, and diakinesis, metaphase I or II of meiosis, short heterochromatic segments are weakly visible or undetectable. In some families with a large amount of heterochromatin, C-bands were observed in the centromeric region in all autosomes during all nuclear division. Some species also show additional heterochromatic regions in the interstitial and/or telomeric regions. In the $\mathrm{X}$ chromosome, C-positive segments are usually present in the centromeric region or distributed along the chromosome, while the $\mathrm{Y}$ chromosome is often completely euchromatic. Large heteropycnotic parts were observed in the mealworm beetle, Tenebrio molitor, and other tenebrionids, and also in species from the genera Bembidion and Trechus (Carabidae) which show very conspicuous blocks of procentric C-bands in almost all chromosomes.

\section{PO:06:12}

\section{Chromosomal characterization and physical mapping of the ribosomal genes (5S and 28S) of Chilean flounder (Paralichtys adspersus).}

\section{N. Lam ${ }^{1}$, P. Iturra ${ }^{2}$ and A. Diaz}

${ }^{1}$ Universidad Andrés Bello, Facultad de Ecologia y Recursos Naturales. Republica 252, Santiago, Chile; ${ }^{2}$ Universidad de Chile, Facultad de Medicina, ICBM, Independencia 1027, Santiago, Chile

The Chilean flounder $P$. adspersus is distributed from Paita in Peru until Lota in Chile. Due to their high commercial value is an important resource for fishing sector and now it is carried out their experimental farm. The knowledge of this species is restricted to ecological, nutritional and reproductive aspects. No genetic studies have been done before.

As a step toward the genetic knowledge of P. adspersus in this work we describe their karyotype by means of classic and molecular cytogenetic techniques. Paralichtys genera is formed of 35 species and it is known the $2 \mathrm{n}$ in $P$. olivaceus and $P$. lethostigma.

In chromosomes obtained by lymphocytes culture, the Giemsa stain and CMA3 banding was used, as well as major (28S and $18 \mathrm{~S}$ ) and minor (5S) rDNA probes were located by FISH.

$P$. adspersus possesses $2 \mathrm{n}=46$, with one metacentric and 22 telocentric pairs (NF 48). This karyotype differs with the reported for the other two members of this genera, with 48 telocentric chromosomes. The conservation of the NF 48 indicates the occurrence of Robertsonian rearrangements in the Paralicthys genome. The NOR bearing pair is telocentric, but we found a third NOR bearing chromosome in some individuals. Like in the most of teleosts the $28 \mathrm{~S}$ and $5 \mathrm{~S}$ ribosomal genes were located in separate loci being the $5 \mathrm{~S}$ located in a small telocentric pair.

The cytogenetic information obtained in this work is a necessary step for the establishment of triploid and homozygote lines in this species.

Grant FONDEF D02I 1094

PO:06:13

Restriction enzyme digestion chromosome banding in Crassostrea and Ostrea species: Comparative karyological analysis within Ostreidae

\author{
A. Leitao', R. Chaves ${ }^{2}$, S. Santos ${ }^{2}$, \\ H. Guedes-Pinto ${ }^{2}$ and P. Boudry ${ }^{3}$ \\ ${ }^{1}$ Institut National des Sciences et Technologies de \\ la Mer, Tunisia; ${ }^{2}$ Department of Genetics and \\ Biotechnology, ICETA-UTAD, Portugal; \\ ${ }^{3}$ IFREMER, Station de La Tremblade, France
}

Reliable banding techniques are one of the major needs to develop the genetic research in oysters. 
In this study, we have carried out the cytogenetical characterization of four oyster species (family Ostreidae) using restriction endonucleases treatments. Chromosomes were treated with three different restriction enzymes (REs), stained with Giemsa, and examined for banding patterns: Crassostrea gigas $(2 \mathrm{n}=20$, total number of bands with ApaI: 74, HaeIII: 61, PstI: 76), Crassostrea angulata $(2 \mathrm{n}=20$, total number of bands with ApaI:62, HaeIII:61, PstI: 55 ) (sub-family Crassostreinae) and Ostrea edulis $(2 \mathrm{n}=20$, total number of bands with ApaI: 82, HaeIII: 59, PstI: 66), Ostrea conchaphila $(2 \mathrm{n}=20$, total number of bands with ApaI: 68, HaeIII: 62, PstI: 69) (subfamily Ostreinae). The treatment of samples with ApaI, HaeIII and PstI REs produced specific banding patterns, which demonstrate the potential of these enzymes for chromosome banding in oysters. This is of special interest since it has been recently shown in mammal chromosomes that restriction enzyme banding is compatible with fluorescent in situ hybridisation. This study therefore provides a fundamental step in genome mapping of oysters, since the chromosome banding with restriction enzymes will facilitate physical gene mapping in these important aquacultured species. The analysis of the banded karyotypes revealed a greater similarity within the genera of Crassostrea and Ostrea than between them.

\section{PO:06:14}

\section{Chromosomal mapping of core histone genes in mussels}

\section{Perez-Garcia ${ }^{1}$, P. Moran ${ }^{1}$ and J. Pasantes ${ }^{1}$ \\ ${ }^{1}$ University of Vigo, Spain}

The class Bivalvia includes some of the bestknown marine invertebrate species, many of which are commercially harvested around the world. Most chromosome studies of the family Mytilidae have been performed using classical cytogenetic techniques and there are only a few works using fluorescent in situ hybridisation (FISH).

In the genus Mytilus the core histone genes are arranged as regular gene repeats of all four core histones (H4, H2B, H2A and H3). The repeats do not always contain linker histone $(\mathrm{H} 1)$ genes. In order to map the core histone genes in Mytilus galloprovincialis (Lamarck 1758) and Mytilus edulis platensis (d'Orbigny 1846) by FISH, chromosome preparations were obtained from gill and gonadal tissues of juvenile mussels, and from whole embryos and larvae, after hypotonic treatment and fixation with methanol/acetic acid. Probes for $\mathrm{H} 2 \mathrm{~B}-\mathrm{H} 2 \mathrm{~A}$ and $18+28 \mathrm{~S}$ rDNA internal transcribed spacers (ITS) were generated by PCR.

Core histone gene clusters are located in two middle sized chromosome pairs, a metacentric pair and a submeta/subtelocentric one. These gene clusters are close to the centromere on the short arm of the metacentric chromosome and in the subtelomeric region of the submeta/sutelocentric long arm. This submeta/subtelocentric chromosome pair is none of the two NORbearing chromosomes of these species.

\section{PO:06:15}

\section{The karyotype of the wild asses (Equus hemionus Khur), one of the two types of donkeys in India}

\author{
J. Solanki ${ }^{1}$, R. Sabapara ${ }^{1}$, S. Bheemineni ${ }^{2}$, \\ R. Uppala ${ }^{2}$ and R. Uppala ${ }^{2}$ \\ ${ }^{1}$ Department of Animal Genetics and Breeding, \\ Veterinary College, Gujarat Agriculture \\ University, Anand-388001; ${ }^{2}$ Green Cross Blood \\ Bank \& Genetic Research Centre, Anil Kunj, \\ Paldi, Ahmedabad-380014, India
}

The Indian wild ass (Equus hemionous Khur) widely known by the local name: Ghurkhur is found in the little Rann of Kutch desert on the kathiawadi peninsula of Gujarat state, India. They are living in a small population and adult animals are study, medium sized, ranging in height from $110-120 \mathrm{~cm}$ at shoulder and about $200 \mathrm{~kg}$ of the body weight. They are light gray to almost white in color with a dark brown or bright yellow sandy line extending down the back to the root of the tail. Other markers include short ears with fawn colored patches on the shoulder, saddle and sides up to the rump. Cytogenetic investigations were carried out on two males and two female wild asses. Chromosomal preparations were obtained from 
peripheral heparinized blood drawn from jugular vein by standard culture techniques followed by Giemsa (GTG), centromeric (CBG) banding and Ag-NOR staining techniques were performed to identify the chromosomes. Analysis of chromosome preparations indicates that the diploid chromosome number is $2 \mathrm{n}=56$ and a fundamental number NF is 100 . This includes 54 autosomes (42 metacentric, submetacentric and 12 acrocentric autosomes), a submetacentric $\mathrm{X}$ chromosome and a small $\mathrm{Y}$ chromosome, which appears to be metacentric. In the female, both the $\mathrm{X}$-chromosomes were homologous and did not show any polymorphism. It was not possible to identify G-banded pattern of the Y chromosome on contracted preparations. To the best of our knowledge, this is the first report on the banded karyotype of this relatively rare and endangered species.

\section{Centromeres Symposium}

\section{L18}

\section{Aurora and Borealin: Not poles apart in the mitotic spindle}

\section{R. Gassmann ${ }^{1}$, A. Carvalho', A. Henzing ${ }^{1}$, S. Ruchaud ${ }^{1}$ and W. Earnshaw ${ }^{1}$}

\author{
${ }^{1}$ Wellcome Trust Centre for Cell Biology, \\ University of Edinburgh, Scotland, UK
}

Chromosomes lacking functional condensin lack a chromosome scaffold fraction. One downstream consequence of this is that the structure of the chromosomes is defective and they are highly sensitive to hypotonic swelling, and lack a structural 'memory'. Thus non-histone proteins of the chromosome scaffold fraction have an essential role in mitotic chromosome structure.

In order to characterise chromosome scaffold components in greater detail, we isolated mitotic chromosomes from human cells. Chromosome scaffolds prepared from this fraction were subjected to SDS-PAGE, and 42 bands excised from the gel were subjected to MALDI-tof analysis. This analysis identified 85 candidate proteins that were likely to be bona fide components of the chromosome scaffold. Of these, 39 were known chromosomal, 8 were likely contaminants, 38 were known, but not described in mitosis and 16 were uncharacterised. Of this latter group, we have tagged three with GFP and shown all of them to be chromosomal. Two appear to be nucleolar proteins that coat the chromosome periphery in mitosis. The third tagged component turned out to have a distribution in cells identical to the chromosomal passenger complex of INCENP, Aurora B and survivin. Studies to be presented indicate that the novel protein, which we call Borealin, is a component of the complex that is essential for normal mitotic progression. These results suggest that the chromosomal passenger complex is likely to be significantly more elaborate than has heretofore been appreciated.

\section{L19}

\section{Molecular dissection of the kinetochore-microtubule interface in Caenorhabditis elegans}

\author{
I. Cheeseman', S. Niessen ${ }^{2}$, J. Yates ${ }^{2}$, \\ K. Oegema ${ }^{1}$ and A. Desai ${ }^{1}$ \\ ${ }^{1}$ Ludwig Institute for Cancer Research/UCSD; \\ ${ }^{2}$ The Scripps Research Insitute, CA, USA
}

Kinetochores play a crucial role in chromosome segregation by mediating attachments between chromosomes and spindle microtubules. To identify novel components of the Caenorhabditis elegans mitotic kinetochore, we have taken a biochemical approach by purifying native kinetochore complexes from worm protein extracts. Using this approach, we identified a 10-subunit KNL-1/3 complex that is essential for kinetochore-microtubule attachments. Functional analysis of each subunit and determination of their kinetochore localization requirements indicates that the KNL-1/3 complex is primarily composed of three distinct functional modules: KNL consisting of KNL-1 and KNL-3, ãMISä consisting of MIS-12, KBP-1, and KBP2, and ãNDCä, consisting of NDC-80, Nuf2(HIM-10), and Spc25(KBP-3). KNL subunits are essential for the assembly of the outer domains of the kinetochore, ãMISä subunits control the rate and extent of formation of these outer domains, and 
'NDC' subunits are necessary to sustain tension at the kinetochore-microtubule interface. The wide conservation of subunits from each module implies that the functions and physical interactions defined we have defined for the KNL-1/3 complex will be relevant throughout the eukaryotic kingdom.

\section{L21}

\section{Chromosome condensation without condensin}

\section{P. Vagnarelli}

Wellcome Trust Centre for Cell Biology, University of Edinburgh, Mayfield Road, Edinburgh EH9 3JR, Scotland, UK

No abstract was submitted for this talk.

\section{L22}

Bub1 and Aurora cooperate to prevent aneuploidy by maintaining BubR1 mediated inhibition of the APC/C

C. Morrow ${ }^{1}$, V. Johnson' ${ }^{1}$, C. Ditchfield ${ }^{1}$ and S. Taylor ${ }^{1}$

${ }^{1}$ School of Biological Sciences, University of Manchester, UK

The spindle checkpoint prevents aneuploidy by inhibiting Cdc20 mediated activation of the anaphase promoting complex/cyclosome (APC/C) until all the chromosomes correctly align on the microtubule spindle via their kinetochores. BubR1, an essential component of this checkpoint, localises to kinetochores and its kinase activity is regulated by the kinesin related motor protein CENP-E. BubR1 also inhibits APC/C$\mathrm{Cdc} 20$ in vitro, thus providing a molecular link between kinetochore-microtubule interactions and the proteolytic machinery that regulates mitotic progression. Several other protein kinases, including Bub1 and members of the Ipl1/ Aurora family, also regulate anaphase onset. However, in human somatic cells Bubl and Aurora kinase activity do not appear to be essential for spindle checkpoint function: when Bub1 is repressed by RNAi or when cells are exposed to Aurora inhibitors, cells arrest following spindle destruction. However, we have recently discovered that when Bub1 and Aurora are simultaneously inhibited, the checkpoint is defective. Furthermore, both Bub1 and Aurora activity are required to maintain BubRlas interaction with the APC/C. One explanation for these observations is that the checkpoint is composed of two arms, one dependent on Bub1, the other on Aurora kinase activity, with both of these arms converging on BubR1, promoting its ability to bind APC/C-Cdc20. We suggest that these two arms respond to different spindle cues: while the Bub1 arm monitors kinetochore-microtubule attachment, the Aurora arm monitors biorientation. This bifurcation in the signalling mechanism may help explain why aneuploid tumour cells mount a robust checkpoint response following spindle damage despite exhibiting chromosome instability.

\section{L23}

\section{Formation of kinetochores and heterochromatin structures in vertebrate cells}

\section{T. Fukagawa ${ }^{1}$}

National Institute of Genetics, Mishima, Schizuoka, Japan

The centromere is a component of the chromosome that is required for accurate segregation of chromosomes during mitosis in eukaryotes. Centromere functions include sister chromatid cohesion and separation, microtubule attachment, chromosome movement, formation of heterochromatin structures and mitotic checkpoint control. Because problems in chromosome segregation can lead to cancer, aneuploidy, and cell death, we must understand the mechanism by which centromeres are formed and interact with the microtubules of the spindle apparatus during cell division. To understand the molecular mechanism underlying centromere function, we used hyper-recombinogenic chicken B lymphocyte cell line, DT40. The high level of homologous recombination in DT40 cells permit efficient targeted modification of genes of 
interest. We have improved this system and have created several cell lines with conditional knockouts of several centromere proteins to investigate the molecular mechanism of kinetochore assembly and centromere function. Here we talk about phenotype of several mutants and discuss about mechanism of kinetochore assembly. In addition to kinetochore assembly, it is important to understand how heterochromatin structure is formed in centromere region of verterbrate cells. In this viewpoint we generated a conditional lossof-function mutant of Dicer in a chicken-human hybrid DT40 cell line that contains human chromosome 21. We will discuss about implication of RNAi machinery for formation of heterochromatin in vertebrate cells.

\section{L24}

\section{Centromeric chromatin demonstrates a distinct spectrum of histone modifications}

\author{
B. Sullivan ${ }^{1}$, A. Lam ${ }^{1}$ and G. Karpen ${ }^{2}$ \\ ${ }^{1}$ Dept of Genetics and Genomics, Boston \\ University School of Medicine, USA; ${ }^{2}$ Dept of \\ Genome Science, Lawrence Berkeley National \\ Lab and Dept of Mol and Cell Biology, \\ University of California, Berkeley, CA, USA
}

Post-translational histone modifications are thought to regulate epigenetic switching between different chromatin states. In fact, distinct histone modifications have been correlated with discrete functional chromatin domains. Acetylation of histones $\mathrm{H} 3$ and $\mathrm{H} 4$, and $\mathrm{H} 3$ methylation at lysine 4 (K4), are predominantly associated with euchromatin. H3 K4 di-methylation (H3 K4-diMe) is associated with 'permissive' chromatin, and H3 K4 tri-methylation (H3 $\mathrm{K} 4$-triMe) is linked with transcriptional activity. Conversely, H3 K9 di- and tri-methylation (H3 K9-diMe and $\mathrm{H} 3 \mathrm{~K}$ 9-triMe) mark constitutive and facultative heterochromatin, and are associated with stochastic gene silencing (position effect variegation). Centromeric (CEN) chromatin is frequently located in or near heterochromatin, and contains blocks of histone-H3 nucleosomes interspersed with blocks of CENPA nucleosomes, the $\mathrm{H} 3$ variant that provides a structural and functional foundation for the kinetochore. We have used extended chromatin fibers and metaphase chromosomes from cultured human cells and Drosophila cells and larval neuroblasts to study histone modifications within centromere regions as markers for the chromatin state within these domains. H3 K9diMe and $\mathrm{H} 3 \mathrm{~K}$ 9-triMe were not present within CEN chromatin, and only H3 K9-diMe was observed in regions immediately flanking the CEN. Surprisingly, H3 K4-diMe staining was observed between CENP-A/CID subdomains in both humans and fly centromeres. We conclude that centromeric nucleosomes contain histone modifications previously associated with both euchromatin and heterochromatin, but in a combination that is distinct from each chromatin state individually. This modification pattern may contribute to the unique domain organization and 3D structure of centromeric regions, and/or to epigenetic information that determines centromere identity.

\section{L25}

\section{Specific DNA modifications are required for CENP-A assembly}

\section{S. Zeitlin' ${ }^{1}$, S. Patel ${ }^{1}$ and G. Slupphaug ${ }^{2}$}

${ }^{1}$ University of California, San Diego, USA;

${ }^{2}$ NTNU, Trondheim, Norway

DNA sequences in metazoan centromeres are not conserved. In spite of this, there is a conserved protein component of metazoan centromeres. The essential, centromere-specific histone H3 variant, CENP-A, is required for kinetochore formation. To determine how CENP-A is specifically assembled at centromeres, we developed a binding assay using sperm chromatin in cell-free extract derived from Xenopus eggs. We show that the catalytic activities of APOBEC $3 \mathrm{G}$, a deoxycytidine deaminase, and UNG2, a uracil DNA glycosylase, are required to create a stable binding site for CENP-A. In support of this model, inhibiting deoxycytidine deaminase with zebularine, or uracil DNA glycosylase with Ugi, uracil or UTP results in a lack of DNAbound CENP-A. Our data suggest that specific 
DNA modifications are required for CENP-A assembly.

\section{L26}

\section{New proteins at the metaphase mammalian centromere}

J. Craig' ${ }^{1}$ P. Canham ${ }^{1}$, E. Earle ${ }^{1}$ and A. K. Choo ${ }^{1}$

${ }^{1}$ Chromosome Research Group, Murdoch Childrens Research Institute \& Dept. of Paediatrics, University of Melbourne, Royal Children's Hospital, Parkville, Melbourne, Victoria 3052, Australia

The centromere, identifiable as the primary constriction on a metaphase chromosome, is an essential structure that coordinates the correct segregation of chromosomes during cell division. Centromeres contain kinetochores, the structures responsible for microtubule capture and spindle attachment. Centromeres also contain heterochromatin, which has been shown to be involved in gene silencing and chromatid cohesion. A large number of kinetochore and heterochromatin-associated proteins have so far been localised to mammalian centromeres and further characterised using dicentric and neocentric chromosomes. However, the role of some of these proteins at centromeres is not understood; many do not occur exclusively at centromeres and this suggests that the centromeres and certain regions within euchromatin share epigenetic marks and macromolecular complexes. To help define these relationships, we have performed a large scale immunofluorescence analysis using antibodies to proteins which, by association with known centromere proteins and with known macromolecular chromatin-related complexes, may be present at mitotic centromeres. We have performed this analysis on Colcemid-arrested mitotic chromosomes from mouse and human cell lines, including those containing neocentromeric and dicentric chromosomes. A number of existing proteins were shown to be exclusively present or enhanced at mammalian centromeres and fell into two broad groups of kinetochore- or heterochromatin-enhanced. The implications of these results will be discussed.
L27

An increase in scaffold/matrix association is an epigentic event that accompanies neocentromere formation

\author{
H. Sumer ${ }^{1}$, J. Craig' ${ }^{1}$ M. Sibson ${ }^{1}$ and \\ A. K. Choo ${ }^{1}$
}

${ }^{1}$ The Murdoch Childrens Research Institute \& Dept. of Paediatrics, University of Melbourne, Royal Children's Hospital, Flemington Road, Parkville, Melbourne, Victoria 3052, Australia

Human neocentromeres arise at previously noncentromeric loci in the human genome. They are functionally equivalent to alpha-satellite based centromeres both in terms of mitotic stability and centromere protein association. We have begun a systematic dissection of chromatin structure at human neocentromeres, which have proven to be valuable tools for dissecting centromere biology. Using a novel method which combines Scaffold/Matrix Attachment Region (S/MAR) DNA isolation and genomic array hybridization, we have identified a $\sim 2.5 \mathrm{Mb}$ region of densely packed chromatin surrounding the core neocentromere of a marker chromosome and confirmed this by fluorescence in situ hybridization on saltextracted metaphase chromosomes. These results suggest that the dense S/MARs seen at normal centromeres are not just a consequence of the tandemly repetitive nature of alpha-satellite DNA, but have an important role in the epigenetic maintenance of centromere structure and function.

\section{L28}

\section{Functional mapping of an American trypanosome centromere}

\author{
J. Kelly ${ }^{1}$, S. Obado ${ }^{1}$ and M. Taylor ${ }^{1}$ \\ ${ }^{1}$ London School of Hygiene and Tropical \\ Medicine, London, UK
}

The trypanosomatids are an ancient family that diverged from the main eukaryotic lineage early in evolution. They include several species of parasitic protozoa that are major public health problems in many parts of the developing world. 
Although genome sequencing is now complete, chromosomal elements that mediate segregation in these organisms have not been identified. In this report we will describe the functional mapping of a centromere in the American trypanosome, Trypanosoma cruzi, a parasite with an unusual mechanism of the genetic exchange that involves the generation of aneuploidy by nuclear hybridisation. Using telomere-associated chromosome fragmentation, we show that the region required for the mitotic stability of chromosome 3 encompasses a transcriptional strand-switch domain and a $16 \mathrm{~kb}$ GC-rich island. This region has been fully sequenced and contains several degenerate retrotransposon-like insertions but atypically, lacks the arrays of satellite repeats that characterise many eukaryotic centromeres. This may represent a paradigm for centromere organisation in trypanosomes and other primitive eukaryotes.

\section{PO:06:16}

\section{Two new cases of neocentric marker chromosomes (NMCs): molecular cytogenetic and clinical characterisation}

\author{
M. Constantinou ${ }^{1}$, E. Zajac ${ }^{1}$, I. Plowas ${ }^{1}$ \\ and B. Kaluzewski ${ }^{1}$ \\ ${ }^{1}$ Department of Medical Genetics, Medical \\ University of Lodz, Sterling str. 1/3, \\ 91-425 Lodz, Poland
}

Extra Structurally Abnormal Chromosomes (ESACs) without detectable alphoid DNA sequences represent a rare and interesting class of rearranged marker chromosomes. These ESACs are predicted to have a neocentromere and have been referred to as Neocentric Marker Chromosomes (NMCs). We report molecular cytogenetic and clinical characterization of two new cases of NMCs (DAPI-, NOR-), one originated from chromosome 1 and the other ö from chromosome 15. Both cases were examined, using FISH technique with alpha-satellite DNA probes specific for chromosomes 1 and 15 and a pancetromeric probe; no alphoid DNA was detected. Only the application of $\mathrm{CGH}$ and $\mathrm{M}-\mathrm{FISH}$ techniques made it possible to determine the chromosomal origin of those chromosomal markers. In case 1, diagnosed prenatally, the NMC originated from the short arm of chromosome 1, comprising two bands (1q21-q22). Autopsy, performed after intrauterine foetal atrophy on the 24 th week of gestation, demonstrated mild intrauterine growth retardation while neither congenital anomalies nor malformations were detected. Case 2 was diagnosed postnatally, demonstrating increased postnatal growth, obesities and a few features if facial dysmorphism. Molecular cytogenetic analysis by $\mathrm{CGH}$ technique revealed amplification of only one chromosome band (15q22) from the distal arm of chromosome 15. The MFISH technique confirmed the origin of NMC. The presented chromosome markers are among the smaller human NMCs described in literature. Additionally, case 2 is the first case of NMC, as described in literature, with such chromosomal breaking points.

\section{PO:06:17}

\section{Fusions of GFP to the separate SAF-A/hnRNP U domains confirm computer analysis of the SAF-A domain structure}

\author{
A. Kukalev ${ }^{1}$, O. Podgornaya ${ }^{1}$ and \\ P. Percipalle ${ }^{2}$ \\ ${ }^{1}$ Institute of Cytology RAS, Saint-Petersburg, \\ Russia; ${ }^{2}$ Karolinska Institutet, Stockholm, \\ Sweden
}

SAF-A/hnRNP $\mathrm{U}$ is an abundant nuclear protein, which has been originally described as nuclear matrix protein. SAF-A is known as protein binding satellite DNA in centromeric (CEN) region, S/MARs DNA sequences and RNA, as component of ribonuclear particles. Immunofluorescence experiments revealed three types of the localization on methaphase plates according to this activities: a) outside chromosomes in residual nuclear matrix b) dots on chromosome arms, c) main signal is in the CEN region. Immunoblot confirms the significant loss of SAF$A$ in the isolated chromosomes contrary to CENP-B, another satellite DNA binding protein. 
The computer analysis has been done in order to understand how the observed complex pattern of the SAF-A localization correlates with its properties and functional domains. In addition to the known ones, a potential NTP-binding region and coiled coil domain have been found.

Three protein's domains revealed by computer analysis were selected for GFP-fusion proteins construction and investigation of their behavior. This is: a) DNA-binding domain, 1-250 a/a; b) potential NTP-binding domain, 250-550 a/a; c) RNA-binding domain, 550-823 a/a. Patterns of their localizations correspond to theoretically predicted. Constructs without DNA-binding domain do not colocalized with heterochromatin defined according to DAPI staining. C-terminal domain with RNA-binding activity could be found in nucleolus. The functional role of the separate SAF-A domains is discussed.

\section{PO:06:18}

\section{Cloning and functional characterisation of plant kinetochore proteins (KP)}

I. Lermontova ${ }^{1}$, S. Hudakova ${ }^{1}$, V. Schubert ${ }^{1}$, R. Manteuffel ${ }^{1}$, B. Schlesier ${ }^{1}$, A. Tewes ${ }^{1}$ and I. Schubert ${ }^{1}$

${ }^{1}$ Institute of Plant Genetics and Crop Plant Research (IPK), D-06466 Gatersleben, Germany

We have cloned putative homologues of two constitutive (CBF5, one isoform of SKP1) and three transient KPs (Bub1 and two isoforms of Bub3) of Arabidopsis thaliana.

$\mathrm{CBF}$ / YFP and CBF5/CFP constructs, transiently expressed in Arabidopsis protoplasts, yielded fluorescent signals clustered within and around the nucleolus. A. thaliana was transformed with a CBF5-RNAi construct to knock out CBF5. To detect interacting proteins, a tobacco library was screened in the yeast twohybrid system with CBF5 cloned into the bait vector. For three candidate clones (presumably encoding CBF1, CENP-F and a kinesin-like protein) corresponding Arabidopsis cDNAs were amplified to confirm interaction with CBF5 by yeast two-hybrid screening and in vivo by FRET.
Arabidopsis plants simultaneously transformed with SKP1/CFP and H2B/YFP, showed YFP fluorescence only within the nuclei, and CFP fluorescence in nuclei, cell wall and cytoplasm. This expression pattern might be explained by involvement of SKP1, a part of the ubiquitin-ligase complex, in different cellular processes.

A number of T-DNA insertion mutants for Bub1, Bub3.1, Bub3.2 were selected from Arabidopsis databases. The absence of the corresponding transcripts in homozygous lines was demonstrated by RT-PCR. The mutants revealed no clear phenotype. Bub3.1 and Bub3.2 mutants were crossed to inactivate both isoforms.

Several monoclonal antibodies (expressed in stabile hybridoma lines) that specifically bind to centromeric regions of isolated faba bean and barley chromosomes have been generated. PAGE, Western blot analysis and mass spectroscopy are applied to identify the centromeric antigens recognized by these MAbs.

\section{PO:06:19}

\section{Conserved satellite DNA sequences in the pericentromeric heterochromatin of tenebrionid beetles (Coleoptera, Insecta)}

\author{
B. Mravinac ${ }^{1}$, N. Mestrovic ${ }^{1}$, Z. Pezer ${ }^{1}$, \\ M. Plohl ${ }^{1}$ and D. Ugarkovic ${ }^{1}$ \\ ${ }^{1}$ Ruder Boskovic Institute, Dept. Molecular \\ Biology, Bijenicka 54, 10000 Zagreb, Croatia
}

Pericentromeric heterochromatin, located on all chromosomes, makes a substantial portion of up to $50 \%$ of the genome in many tenebrionid beetles. Molecular and cytogenetical studies reveal tandemly repeated satellite DNAs as the major heterochromatic DNA components. Usually, a single highly abundant satellite DNA co-exists in the heterochromatin with a number of other, low copy number satellites. The same set of satellite sequences is present in related species and the satellites are differentially amplified among the species forming species-specific profiles. In five species of tenebrionid genus Palorus six unrelated satellite DNAs are found. Major, highly abundant satellite is organized in the form of long 
arrays regularly interspersed with the short stretches of low copy number satellites. Such type of organization encompasses the whole pericentromeric, as well as centromeric regions. The peculiar characteristic of Palorus satellite DNAs is their extremely slow sequence evolution resulting in the absence of divergence and species diagnostic mutations. Complete sequence conservation is detected among the species within the genus as well beyond the level of genus, despite separation for 50-60 Myr. This unexpectedly high conservation might be induced by a bias in turnover mechanisms favouring the ancestral sequence in the process of molecular drive. Selective pressure on Palorus satellite DNA sequences can be also suggested. This could be related to their possible role in the formation of chromatin structure characteristic for pericentromeric and centromeric regions.

\section{PO:06:20}

\section{Connective thread between chromosomes}

\section{O. Podgornaya ${ }^{1}$, I. Kuznetsova ${ }^{1}$, A. Shatrova ${ }^{2}$ and A. Dyban ${ }^{2}$ \\ ${ }^{1}$ Institute of Cytology RAS, St. Petersburg, Russia; ${ }^{2}$ Institute of Experimental Medicine RAMS, St. Petersburg, Russia}

Physical connections between the chromosomes have been previously identified and shown to be neither an artifact of colchicine nor of hypotonic treatment. It has been demonstrated that the interchromosome connection in mammalian cells can be completely destroyed with DNAse but when treated with RNAse or pepsin it becomes more rigid. Sill interchromosome connection is not counted as well established structure. We showed by Fluorescence-Activated Cell Sorting (FASC) that (1) FASC spectrums are never free of aggregates: if there is chromosomes - there is aggregates; (2) the durability of connection is comparable with durability of chromosome itself; (3) the aggregates distribution is in agreement with the curve built on supposition that every one of the chromosome is free to associate with each other. FISH and immunoFISH shows that (1) AT rich mouse major satellite (MaSat) and mouse minor satellite (MiSat) are involved in thread formation, former to the lesser extend, in contrast to GC rich mouse satellites MS3 and MS4 (Kuznetsova et al., 2004), which were never found in interchromosome connection on conventional spreads, in chromosome aggregates from FASC or between meiotic prematurely condensed chromosomes; (2) telomeric sequence is never member of the thread on the same preparations; (3) thread are synthesised in the very late $\mathrm{G} 2$ as shown with BrdU incorporation together with immunostaining; (4) main part of the connection went into midbody after mitosis; (5) MiSat binding protein $\mathrm{p} 68 /$ helicase is one of the main protein components of the interchromosome connection.

\section{PO:06:21}

\section{Histone $\mathrm{H} 3$ modifications in the centromeric regions of Arabidopsis thaliana}

\section{F. Shibata ${ }^{1}$ and M. Murata ${ }^{2}$}

${ }^{1}$ CREST, Japan Science Technology Corporation, Kawaguchi, Japan; ${ }^{2}$ Research Institute for Bioresources, Okayama University, Kurashiki 710-0046, Japan

The 180-bp family of tandem repetitive sequences is the major centromeric satellite in Arabidopsis thaliana. Our previous study showed that part of the 180 -bp repeats are able to assemble the centromere-specific proteins such as HTR12 (Arabidopsis centromeric histone $\mathrm{H} 3$ variant) and AtCENP-C (Arabidopsis CENP-C homolog). It was also suggested that ordinary histone $\mathrm{H} 3$ was involved together with HTR12 at the centromeric regions. To investigate those histone $\mathrm{H} 3$ modifications, we performed indirect fluorescence immunolabeling with antibodies against human phosphorylated histone $\mathrm{H} 3$ at Ser10, at Ser28, and dimethylated histone $\mathrm{H} 3$ at Lys9 (H3K9). The H3K9 methylation was observed in nuclei and chromosomes all through the mitotic cycle, but limited to occur on the heterochromatic regions. The immunosignals appeared on the centromeric heterochromatins were overlapped with those from HTR12 and AtCENP-C. The H3K9 methylation signals on extend chromatin fibers 
were linear and long stretched, whereas the HTR12 and AtCENP-C signals appeared preferentially on knobs of the $180-b p$ repeats. In mammals, phosphorylation of histone $\mathrm{H} 3$ at Ser10 is known to occur all through at the mitotic cell division, and estimated to promote chromosome condensation. In $A$. thaliana, however, both phosphorylations at Ser10 and 28 were thought to occur for ordinary histone $\mathrm{H} 3$, and were observed preferentially at the centromeric regions only during late prophase to metaphase. Compared with HTR12, phosphohistone H3 (Ser10) localized at the inner parts of the kinetochores, which suggests that ordinary phospho-histone $\mathrm{H} 3$ has a role in chromatid cohesion.

\section{PO:06:22}

\section{Topoisomerase II and the vertebrate centromere}

J. Spence ${ }^{1}$, L. Alonso-Gonzalez ${ }^{1}$, W. Mills ${ }^{1}$, A. Carpenter ${ }^{2}$, W. Earnshaw ${ }^{3}$, T. Fukagawa ${ }^{4}$, A. Porter $^{2}$ and C. Farr ${ }^{1}$

${ }^{1}$ Department of Genetics, University of Cambridge, Downing St, Cambridge CB2 3EH UK; ${ }^{2}$ MRC Clinical Sciences Centre, Hammersmith Campus, Du Cane Rd, London W12 ONN UK; ${ }^{3}$ Wellcome Trust Centre for Cell Biology, Institute of Cell and molecular Biology, University of Edinburgh, King's Buildings, Mayfield Road, Edinburgh EH9 3LR UK;

${ }^{4}$ National Institute of Genetics and Graduate University for Advanced Studies, Mishima Shizuoka 4118540, Japan

A number of studies have suggested that topo II, while being involved in a wide range of cellular functions, may also have a specific role at the centromere. Molecular support for this has come from the characterisation of topo II cleavage activity at the human $\mathrm{Y}$ and $\mathrm{X}$ centromeres using etoposide, which acts by stabilising the covalent enzyme DNA intermediate resulting in doublestranded DNA breaks. Where a centromere is molecularly well defined it is possible to use pulsed-field gel electrophoresis to map the immobilised DNA breaks. Within the human $\mathrm{X}$ a-satellite array we have localised a single major site of etoposide-sensitive topo II cleavage. Moreover, through systematic manipulation of this locus and the generation of a large series of deletion derivatives we have shown that centromere activity, the inner kinetochore proteins CENP-C and -H and topo II cleavage co-localise to the same sub-domain of the DXZ1 array. Molecular characterisation of this deletion series also revealed that only part of the starting 2.1 Mb DXZ1 array shared by all centric chromosome derivatives recovered is a region, of $<50$ $\mathrm{kb}$, that encompasses the site of topo II cleavage in the starting array. The ability to map topo II activity at the molecular level presents exciting opportunities for the dissection of this enzymeâs centromere-specific role. Our progress in this will be presented.

\section{Genome Organisation Symposium}

\section{L29}

\section{Biochemical dynamics in and around the centromere}

\author{
K. Sullivan ${ }^{1}$, K. Monier ${ }^{1}$ and A. Visser ${ }^{1}$ \\ ${ }^{1}$ The Scripps Research Institute, La Jolla, \\ CA, USA
}

Heterochromatic regions of the chromosomes are distinctive sites thought to play roles in gene silencing, chromosome spatial organization and mitosis During G2 histone H3 becomes highly phosphorylated at serine 7. Phosphorylation initiates in pericentromeric heterochromatin. Initiation does not occur at random, but preferentially at specific chromosomes in a number of cell types. Immunofluorescence demonstrates that this is mediated by preferential recruitment and activation of Aurora B kinase into specific pericentric heterochromatin domains exhibiting extensive methylation of DNA. Recruitment of Aurora B into pericentric regions is abrogated upon treatment with 5-azacytidine or antisense depletion of DNMT1. We hypothesize that one function of heterochromatin in the cell cycle is to serve as a compartment for the assembly of active Aurora B kinase. A second G2 event at centromeres is the initiation of assembly of the 
kinetochore. The centromere-specific histone H3 homologue CENP-A is specifically expressed in G2. To investigate CENP-A assembly, CENPA-GFP plasmid was microinjected into cells at different stages of the cell cycle. CENP-A was able to assemble promiscuously into chromatin at any stage of the cell cycle, indicating that general chromatin assembly factors can recognize CENP-A. However, preferential assembly of nucleosomal CENP-A at centromeres was also observed throughout the cell cycle. This demonstrates that there is a resident mechanism at centromeres that can continuously exchange available CENP-A into centromeres. Centromeres and pericentric heterochromatin are thus sites of regulated assembly of both resident and nonresident macromolecular complexes during the cell cycle.

\section{L30}

\section{The role of nuclear structure in genomic health}

J. Bridger ${ }^{1}$, K. Meaburn ${ }^{1}$, H. Foster ${ }^{1}$, M. Figgitt', G. Bonne ${ }^{2}$, N. Levy ${ }^{3}$, D. Griffin ${ }^{1}$ and I. Kill ${ }^{1}$

${ }^{1}$ Cell and Chromosome Biology Group, Department of Biological Sciences, Brunel University, Cleveland Road, Uxbridge, UB8 3PH, UK; ${ }^{2}$ INSERM UR 582, Institut de Myologie, Groupe Hospitalier Pitié-Salpetriere, 47,

boulevard de l'Hôpital, 75651 Paris Cedex 13, France; ${ }^{3}$ INSERM U491, Faculte de Medecine de la Timone, 13385 Marseille, Cedex 05, France

Chromosomes and specific types of chromatin are positioned into specific areas in an interphase nucleus. This complex organisation is fundamental for the correct functioning of the genome. Chromatin positioning and reorganisation in particular stages of a cell's lifespan depend upon underlying structures in the nucleus that influence the genome in a variety of ways, structurally to controlling transcription.

We have investigated genome organisation in a number of cell lines derived from patients carrying mutations in proteins associated with nuclear structures, i.e. emerin or lamins A/C.
These nuclear envelope related disorders include Emery-Dreifuss muscular dystrophy (AD-, ARand X-linked EDMD), Dunnigan type of partial familial lipodystrophy (FLPD), Charcot-MarieTooth 2(CMT2), Mandibulacral dysplasia (MAD) and Hutchinson-Gilford Progeria Syndrome (HGPS). We have found that genome organisation changes dramatically over time in culture in these cells. We have a number of assays that measure the degree of chromatin disruption in these cells. These include chromosome positioning by fluorescence in situ hybridisation, telomere binding to the nuclear matrix, DNA halo preparations, replication studies and 3-dimensional reconstruction, visualisation and analysis of chromatin domains.

We have also established a model system for differentiation of porcine ex vivo stem cells to cell types affected in laminopathies. Thus, we are able to determine aspects of genome organisation associated with differentiation and study where this process may go wrong in cells with disturbed nuclear structure.

\section{L31}

\section{The chromatin fibre architecture of heterochromatin and euchromatin in the human genome: relationship to genes and their expression}

\author{
W. Bickmore ${ }^{1}$, N. Gilbert ${ }^{1}$, S. Boyle ${ }^{1}$, \\ H. Fiegler ${ }^{2}$ and N. Carter
}

${ }^{1}$ MRC Human Genetics Unit, Edinburgh, EH4 2XU, UK; ${ }^{2}$ The Wellcome Trust Sanger Institute, Hinxton, Cambridge CB10 1SA, UK

Whilst much is now known about nucleosome structures, little is known about higher-order chromatin structures. We present the first biophysical analysis of chromatin fibre structure across the human genome. Compact and open chromatin fibre structures were separated by sucrose sedimentation and their genomic distributions analysed. We show that compact chromatin fibres are enriched at some sites of cytological heterochromatin (C-bands), and in G-bands (euchromatin). However. some regions 
that are thought of as heterochromatic also contain open chromatin structure suggesting that heterochromatin is a heterogeneous structure.

In contrast, the most open chromatin fibres are enriched at the regions of highest density in the human genome (T-bands), even when the genes in these regions are not expressed. We show that many of these regions are also cytologically decondensed, An open chromatin fibre structure could facilitate transcription and we suggest that this may provide a evolutionary constraint to maintain clusters of genes together along chromosomes.

\section{L33}

Chromosome territory arrangements in the cell nucleus: non-random, probabilistic order and its implications for nuclear functions

\section{T. Cremer ${ }^{1}$}

${ }^{1}$ Department Biology II, Ludwig Maximilians University, Munich, Richard-Wagner-Str. 10/I, D-80333 München, Germany

Chromosome territory (CT) arrangements were analyzed in nuclei of postmitotic human diploid fibroblasts (Bolzer et al., 2004). We demonstrate size dependent, non-random distribution of small CTs towards the nuclear center and of large CTs towards the nuclear rim (radial CT order), This pattern deviates strongly from the gene density correlated, radial CT arrangements reported for lymphocyte nuclei and several other cell types. Side-by-side arrangements (neighborhoods) of heterologous and homologous CTs were highly variable in all cell types. We propose the following hypothesis: Cell type specific gene expression and silencing patterns depend on the position of transcribed genes in euchromatic nuclear zones, and of silent genes in heterochromatic nuclear zones (Cremer et al., 2004). In case of a differentiation dependent switch of gene expression, e.g. from an active to a permanently silent state, affected genes may either maintain their nuclear position, while the embedding chromatin structure changes from an 'open' to a 'closed' configuration, or affected genes may move from an euchromatic to a heterochromatic nuclear zone (gene relocation hypothesis).

Bolzer, A., Kreth, G., Solovei, I., Saracoglu, K., Fauth, C., Müller, S., Eils, R., Cremer, C., Speicher, M. R., Cremer, T. (2004) Complete 3D-maps of chromosome positions in human male fibroblast nuclei and prometaphase rosettes demonstrate a chromosome size dependent, probabilistic arrangement (submitted).

Cremer, M., Zinner, R., Stein, S., Albiez, H., Wagler, B., Cremer, C., Cremer T. (2004) Three dimensional analysis of histone methylation patterns in normal and tumor cell nuclei. Eur. J. Histochem. 48: 15-28.

\section{L34}

\section{Large scale chromatin structure and dynamics}

\author{
A. Belmont ${ }^{1}$ \\ ${ }^{1}$ University of Illinois
}

No abstract was submitted for this talk.

\section{L35}

The analysis of long-range compaction and positioning of interphase chromatin in budding yeast by high resolution live imaging

S. Gasser', K. Bystricky', P. Heun ${ }^{2}$, J. Langowski ${ }^{3}$ and H. Schober ${ }^{1}$

${ }^{1}$ University of Geneva, Frontiers in Genetics-NCCR, Geneva, Switzerland; ${ }^{2}$ Lawrence Berkeley Laboratory, Berkeley, CA USA; ${ }^{3}$ German Cancer Research Center, Heidelberg, Germany

Using optimized in situ hypbridization and live imaging techniques we have determined pointto-point compaction ratios for interphase chromatin in budding yeast. Quantitative two color FISH data on sequences found at 14- to $100-\mathrm{kb}$ intervals along single chromatids are compared with 3D measurements of whole chromosome arms ranging from 122 to $623 \mathrm{~kb}$ in length, monitored through the binding of GFP-repressor fusions to targeted arrays of repressor sites. The resulting distance measurements are introduced into flexible polymer modeling algorithms. The results argue that interphase 
chromatin exists in a higher order conformation with a persistence length of $100-150 \mathrm{~nm}$, or $\sim 15$ $\mathrm{kb}$ based on a mass density of 6-7 nucleosomes per $11 \mathrm{~nm}$. This degree of compaction, equivalent to a folded $30 \mathrm{~nm}$ fiber, is remarkable given that all intervals monitored are open, transcriptionally competent chromatin. We propose that this state unfolds transiently as transcription occurs.

Using similar quantitative fluorescence analysis of of GFP-,YFP- and CFP-tagged chromosomal loci we also show a spatially distinct tethering of centromeres and telomeres in the yeast interphase nucleus. By integrating unrelated bacterial operator arrays into unique chromosomal sites we measure telomere-telomere and telomere-spindle pole body distances in living cells. We show that yeast Chr 3, 5 and 6 loop back upon themselves. Unlike the ends of these chromosomes, those of Chr 14 are not clustered together. Results from telomere swapping, analysis of subtelomeric sequences, and telomere interaction in mutant strains will be presented.

\section{L36}

\section{Identification and chromosomal distribution of a human chromosome 9 duplicon mediating meiotic and somatic rearrangements.}

\section{Paulis ${ }^{1}$, D. Moralli ${ }^{1}$, L. De Carli ${ }^{1}$ and} E. Raimondi ${ }^{1}$

${ }^{1}$ Dipartimento di Genetica e Microbiologia 'A. Buzzati Traverso' Universita di Pavia. Via Ferrata, 1. 27100 Pavia, Italy

The origin of an accessory marker chromosome derived from a chromosome 9 rearrangement has been elucidated. Isolation, sequencing and sequence analysis of one of the breakpoints mapping in 9p21 have been performed. We found a complex duplicon, containing the breakpoint, composed of sub-domains with a redundant structure consisting of blocks of repetitive sequences belonging to the Alu and L1 families, DNA transposons and three full-length genes of unknown function. Database search for paralogous segments on chromosome 9 revealed that the same duplicon is present in 9p21.1, $9 \mathrm{p} 11,9 \mathrm{q} 11,9 \mathrm{q} 21$ and in 9q22.3.

A growing body of evidence indicates that a relationship can be established between constitutive rearrangements and acquired somatic aberrations. Particular rearrangement prone chromosome regions show clustering of recurrent cancer breakpoints. These data support the idea that duplicons play a role in driving not only meiotic, but also somatic exchanges. We found a cancer breakpoint hot spot in 9p21 and more than 100 inter-chromosomal tumor rearrangements between 9p21 and 11q23. Sequence analysis demonstrated that band $11 \mathrm{q} 23$ contains a segment displaying a high degree of homology to the duplicon that we isolated from $9 \mathrm{p} 21.1$.

In silico analysis demonstrated that segmental duplications showing more than $90 \%$ similarity to chromosome 9 dupicons are present in 22 loci distributed on chromosomes 1, 2, 3, 4, 5, 6, 11, $12,13,15,16,18,21,22$, and Y. FISH localization confirmed the in silico data.

\section{L37}

\section{Large-scale chromatin organization and gene expression}

P. Verschure ${ }^{1}$, J. Mateos-Langerak ${ }^{1}$, I. Van der Kraan ${ }^{1}$, A. Belmont ${ }^{2}$ and R. Van Driel ${ }^{1}$

${ }^{1}$ SILS, Univ. of Amsterdam, Amsterdam,

The Netherlands; ${ }^{2}$ Dept. of Cell and Structural Biology, Univ. of Illinois, Illinois, USA

Research focuses on the functional organization of chromosomes and chromatin in the interphase cell nucleus. Actively transcribed loci and epigenetically silenced genes locate at condensed chromatin surfaces [1, 2, 3,4]. Condensed chromatin domains are accessible for large macromolecules, implicating that other mechanisms than inaccessibility of heterochromatin regulate gene silencing within heterochromatin [5].

Recently we investigate the inter-relationship between chromatin structure, gene expression and regulatory proteins involved (i.e. HP1) using two strategies: (i) interference with the functionality of HP1, expressing truncated HP1alpha or HP1beta lacking a functional chromodomain, and (ii) in vivo targeting of HP1alpha or HP1beta 
to an integrated amplified chromosome region, using a lac-operator/repressor based system.

Our data indicate that HP1alpha and HP1beta are not the only proteins involved to maintain and reassemble condensed heterochromatin domains, whereas in vivo HP1 targeting causes local chromatin condensation. We aim to unravel the molecular pathways for functional organization of transcriptionally active and epigenetically silenced genes.

1. Verschure PJ, Van der Kraan I, Manders EMM, Van Driel R (1999) J. Cell Biol. 4: 13.

2. CMarko D, Verschure PJ, Martin TE, Dahmus ME, Krause S, Fu X-D, Van Driel R, Fakan (1999) Mol. Biol. Cell. 10: 211.

3. Verschure PJ, Van der Kraan I, Enserink, JM, Mone MJ, Manders EMM, Van Driel R (2002) J. Histochem. Cytochem. 50: 1303.

4. CMarko D, Verschure PJ, Otte AP, Van Driel R, Fakan F (2003). J. Cell Science 116: 335.

5. Verschure PJ, Van der Kraan I, Manders EMM, Houtsmuller AB, Van Driel R (2003) EMBO Reports 4: 861-866.

\section{L38}

\section{Tissue-specificity of spatial genome organization}

L. Parada ${ }^{1}$, P. McQueen ${ }^{2}$ and T. Misteli ${ }^{1}$

${ }^{1}$ National Cancer Institute, NIH, Bethesda, MD 20892, USA; ${ }^{2}$ Mathematical and Statistical Laboratory, DCB, CIT, NIH, Bethesda, MD 20892, USA

Genomes are organized in vivo in the form of chromosomes. The spatial positioning of chromosomes within the cell nucleus is non-random. Patterns of chromosome positioning have been described in lymphocytes and fibroblasts. However, it is unclear how conserved the spatial organization of genomes is amongst tissues. Using 2D and 3D fluorescence in situ hybridization we have investigated whether spatial genome organization is conserved amongst tissues. We show that genomes are differentially organized in different tissues. Chromosomes exhibit tissue-specific radial positioning with respect to the center of the nucleus and relative to each other. Subsets of chromosomes form distinct types of spatial clusters in different tissues and the relative distance between chromosome pairs varies amongst tissues. Consistent with the notion that spatial proximity contributes to translocation frequency, we find a tissue-specific correlation between spatial proximity and tissue-specific translocation prevalence. Our results demonstrate that the spatial organization of genomes is tissue specific and point to a role of spatial genome organization in the prevalence of cancerous chromosome arrangements.

\section{L39}

\section{Chromosome intermingling in the nucleus of human lymphocytes}

\author{
M. Branco ${ }^{1}$ and A. Pombo' \\ ${ }^{1}$ MRC Clinical Sciences Centre, UK
}

The discreteness of chromosome territories has been studied by chromosome painting, known to miss repetitive sequences and less condensed DNA, or through more indirect methods, like CldU/IdU labelling followed by chromosome segregation. We optimised the chromosome painting procedure in ultrathin cryosections ( $\sim 150 \mathrm{~nm}$ thick), and found that they allow the use of harsher treatments while preserving the organisation of chromatin. Painting efficiency could therefore be improved and this led us to question whether DNA from different chromosomes might intermingle. We co-hybridized 9 pairs of chromosomes in activated human lymphocytes and found a measurable degree of intermingling. Interestingly, the degree of intermingling correlated with the frequency of gamma-ray-induced translocations in the same cell type (Cornforth et al., 2002, JCB, 159: 237). Intermingling was also studied in resting and alpha-amanitin-treated (activated) lymphocytes, aiming to assess a possible role for transcription. When compared with activated cells, resting cells showed differences in the degree of intermingling of different pairs of chromosomes, but there was not a general tendency for increase or decrease. Alpha-amanitin treated cells tended to have increased degrees of intermingling, which suggests that transcriptional activity may constrain chromosome territories. In activated lymphocytes, intermingling is not correlated to 
chromosomal volume, probably due to differential positioning of chromosomes within the nucleus. In contrast, intermingling in resting cells can be justified by differences in chromosomal volume alone, suggesting that chromosome position is random. Studies are currently being undertaken to analyse chromosome intermingling at the electron microscope level and to assess preferential intermingling of specific subchromosomal regions.

\section{L40}

\section{Chromosome subdividing to haploid sets in diploid metaphase plates of some mammalian species}

\section{T. Glazko ${ }^{1}$}

${ }^{1}$ Institute of Agriecology and Biotechnology, Ukraine

In investigation of metaphase plates of smallsized and large mammalian species (bone marrow cells of laboratory lines of mice, Microtus arvalis, Microtus oeconomus, Clethrionomys glareolus; cells of peripheral blood of cattle) the space chromosome subdividing on two haploid sets was revealed. The comparative analysis of Robertsonian translocations in bone marrow cells (in vivo) and in cell lines (in vitro) of laboratory lines of mice had shown, that the transition to passing of cells from in vivo to in vitro was accompanied by destruction of chromosome organization in haploid sets. So, the Robertsonian translocations between homologous chromosomes were revealed only in cells in vitro in difference from in vivo populations. It allows to suppose that chromosome haploid set is an autonomous element in organization of a genetic material, which destabilizing is connected with the appearance of some cytogenetic anomaly.

The analysis of polytene chromosome positions in salivary gland cells of Chironomus thummi demonstrated that the chromosome space order can be related with the dynamics of ratios between strength of their attachment to nucleus periphery and between chromosomes. The decreasing of chromosome connections with a nucleus periphery is accompanied by the increase of interchromosome associations; the increase strength of the chromosome attachment to nucleus periphery, accordingly, results in increase of chromosome spatial dissociation. At the same time, in all cases the nucleolus-forming chromosome 4 remained attached to the nucleus periphery. That is, such ratio can be specific to separate chromosome and can participate in maintenance of chromosome order in interphase nuclei.

\section{L41}

\section{When is a chromosome is too large to handle?}

\author{
W. Rens ${ }^{1}$, P. O'Brien ${ }^{1}$, F. Yang ${ }^{1}$ and \\ M. Ferguson-Smith ${ }^{1}$
}

${ }^{1}$ Molecular Cytogenetics Laboratory, Centre for Veterinary Science, Department of Clinical Veterinary Medicine, University of Cambridge, Madingley Road, Cambridge, UK

The extremely long X-chromosome of the field vole, Microtus agrestis, appears to hamper proper nuclear divison. This observation was obtained using FISH with chromosome specific paints, and immuno-fluorescence with an antibody against the nuclear pores and an antibody against INCENP (inner centromere protein). Cells, preserved in their 3D configuration, were investigated by Z-stack fluorescent microscopy at different stages of the cell cycle, from metaphase till furrow formation.

The DNA content of most diploid mammalian cells is around $7 \mathrm{pg}$. However, the DNA is distributed over a different number of chromosomes in different species, and consequently the sizes of these chromosomes vary considerably. The position of the centromere along the chromosome is not fixed either, leading to chromosomes of varying length. At late anaphase chromosomes are pulled apart by microtubules attached to kinetochores at the centromeres, but the space in which the chromosomes can move apart is limited. In plants, artificially elongated chromosomes result in impairment of viability. We have already shown in the potoroo that long chromosomes affect the shape of the nucleus and their relative position in the nucleus (Chromosoma 2003; 112:66-76). The case of the field vole is more extreme. The long $\mathrm{X}$-chromosome leads to DNA bridges between 
nuclei, i.e. incomplete nuclei divison, and the occurrence of micronuclei, i.e. loss of chromosome fragments. Apparently there is no mitotic check- point for lagging chromosome arms. The significance of these findings on the natural history of the field vole remains to be determined.

\section{L42}

\section{Effects of chromosome} rearrangements during primate evolution on the higher order chromatin architecture

\author{
M. Neusser ${ }^{1}$, V. Schwarz', A. Koch' ${ }^{1}$, \\ T. Cremer ${ }^{1}$ and S. Müller ${ }^{1}$ \\ ${ }^{1}$ Department Biology II, Human Genetics, \\ Ludwig-Maximilians University, Munich, \\ Germany
}

It is not known whether certain evolutionary chromosome rearrangements are favoured by distinctly non-random positions of chromosome territories (CT). Depending on specific structural properties of chromosome segments involved in evolutionary rearrangements they may or may not lead to the dislocation of chromosomal material to different nuclear compartments with different functional environments. Considering possible effects of higher order chromatin arrangements on gene activity, it is important to study whether different evolutionary rearrangements do affect the nuclear topology of the respective chromosome segments. This is particularly interesting in cases, where translocations brought together chromosome segments with high and low gene density. We performed 3D FISH studies of interphase nuclei from human and a variety of other primate species in order to trace the influence of chromosomal rearrangements that have occurred during evolution on nuclear organization. These studies address the following questions: Are gene density-correlated radial arrangements of chromatin evolutionary conserved in different species despite extensive evolutionary chromosome reshuffling? Does the CT size of evolutionary translocation products affect their nuclear localization? Is the side-by-side arrangement of rearranged chroma- tin essentially random or not? Does the amplification of heterochromatin and segmental duplications affect the position of neighbouring chromatin segments? We expect these studies to contribute to the detailed analysis of preservation/changes of higher order CT arrangements in various primate evolutionary lineages. On the other hand these experiments may help to clarify whether non-random higher order chromatin arrangements affect the nature and direction of karyotype evolution.

\section{L43}

\section{Spatial position of satellite DNA in respect to chromosome territories in interphase nuclei of human cells}

\author{
N. Enukashvily ${ }^{1}$, R. Donev ${ }^{2}$ and \\ O. Podgornaya ${ }^{1}$
}

${ }^{1}$ Cell Cultures Dept., Institute of Cytology, Tikhoretsky, 4., St. Petersburg, 194064, Russia; ${ }^{2}$ University of Wales College of Medicine, Medical Biochemistry \& Immunology Dept, Biology of the Complement Group, Heath Park, Cardiff CF14 4XN, UK

The existence of chromosome territories (CT) is a well-established fact. The role of satellite DNA of centromeric and pericentromeric regions in nuclear spatial organisation has been also proposed. The aim of our work was to investigate the spatial position of satellite DNA and some proteins bound to it in respect to CTs. In human lymphocytes, placenta parenchyma cells, fibroblasts, A431 and HeLa cells, centromeres revealed by FISH with labelled alphoid DNA were distributed throughout the nuclear interior. Centromeres of some chromosomes were positioned on the surface of CTs as shown by FISH. The spatial proximity of some centromeres to interchromosomal SC35 domains was demonstrated by immunoFISH. Thus, some centromeres can be located at the CT periphery. Human pericentromeric DNA (human satellite 3, HS3) of chromosome 1 but not 14 was always adjacent to nuclear envelope in primary cultures. In HeLa cells we used, 3-4 signals corresponding to chromosome $1 \mathrm{HS} 3$ were revealed by FISH. Only two of them were adjacent to nuclear envel- 
ope. The contacts between HS3 regions of chromosomes 1 and 14 were observed in all cell cultures analysed. HS3 regions of these chromosomes remained always compacted in these cells. In old but dividing MRC 5 fibroblasts culture (passage 30-36), HS3 was unfolded. It's threads were invading neighbour CTs. AB against RNA helicase p68 decorated a network framing CT. Thus CTs have well defined borders, but some chromosomal regions can penetrate into CT of neighbour chromosomes. Satellite DNA is involved in these processes.

\section{L44}

\section{3-D localisation of centromeres and chromocenters in preimplantation mouse embryos}

\section{Merico ${ }^{1}$, M. Monti ${ }^{1}$, M. Zuccotti ${ }^{2}$, C. Redi ${ }^{1}$ and S. Garagna ${ }^{1}$}

${ }^{1}$ Dipartimento di Biologia Animale and Centro di Eccellenza in Biologia Applicata, University of Pavia, Piazza Botta 10, 27100 Pavia, Italy; ${ }^{2}$ Dipartimento di Medicina Sperimentale, Sezione di Istologia ed Embriologia, Parma University, Via Volturno 39, 43100 Parma, Italy

Development requires a precise program of gene expression to be carried out. Epigenetic mechanisms such as DNA methylation, histone acetylation, chromatin organisation and nuclear architecture are involved in modelling and moulding genome functioning. Antral oocytes isolated from the mouse ovary display meiotic and developmental competence which is correlated with their nuclear architecture. Oocytes showing a homogeneous diffused chromatin are incapable of developing beyond the two-cell stage whereas oocytes showing a rim of chromocenters and centromeres surrounding the nucleolus are capable of further development to the blastocyst stage after in vitro fertilisation.

In preimplantation embryos, the highly methylated maternal and the paternal genome are topologically separated up to the four-cell stage (Mayer et al., Nature, 403:501, 2000).

In order to follow the changes of the nuclear architecture in mouse embryos throughout preimplantation development we have determined the 3-D localisation of centromeres, chromocenteres and nucleoli. In two-cell embryos, centromeres and chromocenters cluster in half of nucleus and are localised at its periphery. An average of seven nucleoli are dispersed within the nucleus. At four-cell stage, most of the centromeres and chromocenters are associated with the nucleoli which average number is four. During next stages, nucleoli tend to cluster, as well as their associated centromeres and chromocenters, whereas the others are dispersed throughout the nuclear volume.

Our results are indicative of a dynamic 3-D spatial organisation of the genome and provide the molecular morphology ground necessary for understanding the regulatory mechanisms that control gene expression at different hierarchical levels during preimplantation development.

\section{L45}

\section{Organization and dynamics of plant chromosome territories (Arabidopsis)}

\author{
A. Pecinka ${ }^{1}$, V. Schubert ${ }^{1}$, A. Meister ${ }^{1}$, \\ G. Kreth ${ }^{2}$, N. Kato ${ }^{3}$, M. Lysak ${ }^{1}$, J. Fuchs ${ }^{1}$, \\ M. Klatte ${ }^{1}$, E. Lam ${ }^{3}$ and I. Schubert ${ }^{1}$ \\ ${ }^{1}$ Institute of Plant Genetics and Crop Plant \\ Research (IPK), Corrensstrasse 3, D-06466 \\ Gatersleben, Germany; ${ }^{2}$ Kirchhoff Institute \\ for Physics, University of Heidelberg, \\ Im Neuenheimer Feld 227, D-69120 Heidelberg, \\ Germany; ${ }^{3}$ Biotech Center, Cook College, \\ Rutgers \& The State University of New Jersey, \\ 59 Dudley Rd., Foran Hall, New Brunswick, \\ NJ 08901, USA
}

Differential painting allowed to study organization and potential dynamics of chromosome territories (CTs) in A. thaliana nuclei. The sideby-side arrangement of heterologous chromosomes and the homologous association of the symmetric chromosomes 1,3 and 5 are in accordance with the random frequency predicted by computer model simulations for differently shaped nuclei. Homologues are spatially associated on average in $35-50 \%$ of nuclei and in up to $70 \%$ of spheric nuclei. Only the NOR-bearing chromosome 2 and 4 homologues associate more frequently than at random, apparently due to attachment of NORs to a single nucleolus. Punctual homologous pairing of $\sim 100 \mathrm{~kb}$ seg- 
ments (in $\sim 5 \%$ of nuclei) occurs 7-10 times less frequently than homologue association, not significantly more often than expected at random and not simultaneously along the homologues. Thus, CT arrangement in Arabidopsis differs from that in Drosophila (characterized by somatic pairing of homologues in $60-90 \%$ of nuclei) in spite of similar genome size, sequence organization and chromosome number. Nevertheless, in 9.3-31.5\% of investigated Arabidopsis nuclei allelic sequences may share positions close enough for homologous recombination. The average punctual pairing frequency is approximately doubled at the integration loci in lines transgenic for the lacO/lacI-GFP chromatin tagging system and becomes further increased by expression of the GFP-LacI fusion protein. With increasing endopolyploidy level of nuclei, the number of homologous CTs does not increase due to association of NORs and of homologous pericentromeric chromocenters. However the frequency of sister chromatid cohesion becomes dramatically reduced, particularly at interstitial chromosome arm positions.

\section{L46}

\section{Molecular characterisation of a $t(1 ; 4)$ translocation associated with primary microcephaly}

\section{B. Pichon', S. Vankerckhove', G. Bourrouillou', M. Abramowicz ${ }^{3}$ and L. Duprez ${ }^{1}$}

${ }^{1}$ Laboratoire de Cytogénétique, Hôpital Erasme, Université Libre de Bruxelles, Belgique; ${ }^{2} \mathrm{CHU}$ Purpan, Toulouse, France; ${ }^{3}$ Service de Génétique Médicale, Hôpital Erasme, Université Libre de Bruxelles, Belgique

Reciprocal translocations associated with abnormal phenotypes constitute powerful tools for the study of genotype-phenotype association and the identification of gene function. The molecular mechanisms responsible for these translocations are poorly understood today. Detailed study of these translocations at the molecular level revealed that they are generally associated with cryptic complex rearrangements in the vicinity of the translocation breakpoints. The identification of the rearrangements involved in the translocations should probably help us to better understand the molecular events responsible for these translocations. Here, we report the molecular study of a familial $\mathrm{t}(1 ; 4)(\mathrm{q} 31.3 ; \mathrm{p} 15.32)$ translocation associated with primary microcephaly. We showed that this translocation disrupts the ASPM gene in 1q31.3. Recently, mutations in the ASPM gene have been reported in some cases of familial microcephaly Bond et al. 2002. Nat. Genet. 32:316-320). Although G-Banding karyogramme and $\mathrm{CGH}$ did not reveal any imbalance within the breakpoints, FISH and Southernblotting have shown several rearrangements including duplication, deletion and insertion. We are currently being delineate and sequence these rearrangements in order to propose mechanism for translocation.

\section{PO:06:23}

\section{Characterization of two families of tandem repeated DNA sequences in Potamogeton pectinatus L.}

\author{
M. Ceccarelli ${ }^{1}$, S. Minelli ${ }^{1}$, V. Sarri ${ }^{1}$ and \\ M. Gelati ${ }^{2}$
}

${ }^{1}$ Dipartimento di Biologia Cellulare e Molecolare della Universit, Sezione Citologia e Genetica, Perugia, Italy; ${ }^{2}$ Dipartimento di Agrobiologia e Agrochimica, Universit della Tuscia, Viterbo, Italy

Two families of tandem repeated DNA sequences, PpeRsal and PpeRsa2, have been isolated from a partial genomic DNA library of the submerged macrophyte Potamogeton pectinatus L. (fennel or sago pondweed). The consensus sequences of PpeRsa1 and PpeRsa2 are 365 and 359 base pairs in length, respectively. Both are enriched in adenine + timine $(62,5 \%$ with PpeRsa1 and 59,3\% with PpeRsa2) and do not contain internal repeats. Repeat structure within the two sequence families is well conserved because the nucleotide sequence similarity among the sequenced clones is $92-96 \%$ with PpeRsa1, and 86-99\% with PpeRsa2. No obvious nucleotide sequence similarity occurs between the two repeats, but certain sequence traits may suggest their evolution from a common ancestor. 
No significant nucleotide sequence identity was found after comparing PpeRsa1 and PpeRsa2 with DNA sequences in the EMBL-GenBank. As shown by fluorescent in situ hybridization experiments, the two sequence families are present in 52 out of the 78 chromosomes of $\mathrm{P}$. pectinatus. PpeRsa1-related sequences have a marked preferential localization at the chromosome ends, while PpeRsa2-related sequences occur also at various intercalary chromosome regions.

The two sequence families proved to be useful molecular markers to assess intraspecific variation of the genome size and organization in fennel pondweed. Indeed, their copy number and structure vary significantly among five populations in Central Italy.

\section{PO:06:24}

\section{DNA/DNA interactions may be involved in chromosomal compaction}

\section{Glazkov ${ }^{1}$}

${ }^{1}$ Vavilov Institute of General Genetics, Russian Academy of Sciences, Russia

A possible mechanism of compactization of eukaryotic chromosomes with the involvement of triple-stranded DNAs was studied by using computer modelling carried out for analysis of nucleotide sequences of 17 eukaryotic genes, satellite DNAs as well as LINEs and SINEs.

The obtained results indicate that chromosomal regions containing genes as well as satellite DNAs, LINEs and SINEs are capable of compactization into small loops. Chromosomal domains of genes often form rosette-like structures. The fact that gene domains of animal and plant chromosomes are able to compact into 'isolated' series of loops can serve as a structural basis of their independent expression. Rosette-like structures have been previously found in somatic and meiotic chromosomes by cytological methods. It has also been established earlier that these structures are form of organization of transcription inactive genes. The results suggest that protein noncoding regions of chromosomal domains of genes (flanks and introns of genes) carry information about the mechanisms of their compactization.
Analysis of satellite DNAs has demonstrated a fundamental similarity of the mechanisms of compactization of chromosomal DNA regions encoding and not encoding proteins. In the latter case, however, a more comlicated and more considerable compactization of tandem repeats of satellite DNAs is observed. This is in agreement with cytological data on a greater degree of compactization of chromosomal parts which contain extended regions of satellite DNAs (constitutive heterochromatin) as compared to euchromatin.

The work was supported by an RFBR grant.

\section{PO:06:25}

\section{An Investigation on status of tylosis with oesophageal cancer (TOC) locus in Iranian patients afflicted with oesophageal squamous cell carcinoma}

M. Noori Daloii ${ }^{1}$, M. Shahabi ${ }^{2}$, E. Jahanzad ${ }^{3}$, E. Khoshbin ${ }^{4}$, M. Taghikhani ${ }^{2}$, J. Field ${ }^{5}$, J. Langan ${ }^{5}$ and J. Risk ${ }^{5}$

${ }^{1}$ Department of medical Genetics, Faculty of Medicine, Tehran University of Medical Sciences, Tehran, Islamic Republic of Iran; ${ }^{2}$ Pasteur Institute of Iran, Tehran, Islamic Republic of Iran; ${ }^{3}$ Cancer Institute, Imam Khomeini Hospital, Tehran, Islamic Republic of Iran; ${ }^{4}$ Department of Mathematics, Faculty of Science, University of Tehran, Tehran, Islamic Republic of Iran; ${ }^{5}$ Department of medical Genetics, Faculty of Medicine, Tehran University of Medical Sciences, Tehran, Islamic Republic of Iran; 2Molecular Genetics and Oncology Group, Department of Clinical Dental Sciences, University of Liverpool, Liverpool L69 3BX, UK

Tylosis (hereditary hyperkeratosis palmaris et plantaris) is an autosomal dominant disease characterized by thickening of the skin that is associated with a very high risk of esophageal squamous cell carcinoma (ESCC) in three pedigrees investigated. Haplotype and linkage analyses using microsatellite markers have previously mapped the tylosis with oesophageal cancer (TOC) locus to a $500 \mathrm{~kb}$ region on $17 \mathrm{q} 25$. $\mathrm{LOH}$ of markers within this region have been found in sporadic cases of ESCC. Further fine mapping of 
the TOC locus within tylotic families using additional microsatellite and SNP markers reduced the TOC minimal region to $42.5 \mathrm{~kb}$. In the present study we investigated loss of heterozygosity (LOH) of markers spanning 17q25, three of which were within the TOC minimal region, in 60 Iranian ESCCs. LOH patterns ranged from complete loss of $17 \mathrm{q} 25$ to small deletions within the minimal region and to no deletion at all. Seventy three percent $(44 / 60)$ of patients had $\mathrm{LOH}$ in at least one marker within the $17 \mathrm{q} 25$ region and all markers tested had more than 50\% LOH. One known and two putative genes lie within the minimum deletion region, including the cytoglobin gene. We performed a mutation analysis on exons of the cytoglobin gene using single strand conformational polymorphism (SSCP) and DNA sequencing. We found a few mutations which suggests that this gene may be involved in the ESCC phenotype.

\section{PO:06:26}

\section{Physical mapping with}

high-throughput fingerprinting for low-molecular-weight glutenin loci (Glu-3) in hexaploid wheat (Triticum aestivum)

\section{N. Ozdemir ${ }^{1}$ and S. Cloutier ${ }^{2}$}

${ }^{1}$ Yildiz Technical University, Department of Biology, Molecular Biology, Davutpasa str. 127 Esenler, Istanbul, Turkey 34210; ${ }^{2}$ Cereal Research Center, Agriculture and Agri-Food Canada, 195 Dafoe Road, Winnipeg, Manitoba, Canada R3T 2M9

Glutenins are important storage proteins of wheat and help determine bread-making quality. They contain different polypeptides, which are subdivided into high molecular weight (HMW) and low molecular weight (LMW) categories. Genes tightly linked to Glu-A3, Glu-B3, and GluD3 on the short arms of chromosomes 1A, 1B and 1D, respectively, control most of the LMW glutenin subunits. Three developing seed cDNA libraries were constructed and a total of 50006000 ESTs were created from these libraries. ESTs were assembled into contigs and searched by homology for LMW glutenin sequences. 90 full-length LMW glutenin sequences were defined that clustered into 8 distinct groups representing at least 20 different LMW glutenin subunits. A LMW glutenin composite probe was used to screen a hexaploid wheat BAC library by hybridizing a set of 24 high-density filters. A subset of 536 BAC clones was selected and fingerprinted as a result of two rounds of hybridization. A set of 25 pairs of PCR primers was designed from fulllength LMW glutenin sequences and used as markers on the BAC clones. The combined fingerprinting and marker data was used to build the physical maps using FPC. The total length of the 90 contigs was $14.5 \mathrm{Mb}$; the average contig comprised three BAC clones and was $161 \mathrm{~kb}$ long. The longest contigs comprised 14 and 11 clones and spanned $403 \mathrm{~Kb}$ and $343 \mathrm{~Kb}$ respectively.

\section{PO:06:27}

\section{Characterization and chromosome location of a repetitive DNA and a mariner-like element in the ant Tapinoma nigerrimum}

\author{
T. Palomeque ${ }^{1}$, J. Carrillo ${ }^{1}$, M. Muñoz ${ }^{1}$ \\ and P. Lorite ${ }^{1}$ \\ ${ }^{1}$ Departamento de Biologia Experimental, \\ Universidad de Jaén, Spain
}

Genomic DNA from $T$. nigerrimum digested with HindIII originates a single 200-bp band (TANI), which has been sequenced. Southern blotting using TANI as a probe did not reveal the ladder pattern typical of tandemly repetitive DNA. Using the TANI sequence, we have designed the primers and carried out PCR amplification. The PCR results show that it produced a single amplification band of $1 \mathrm{~kb}$ (TANIPCR). Southern blotting using this sequence as a probe also produced the ladder pattern typical of tandemly repetitive DNA. These sequences did not show significant similarity to sequences previously deposited in GenBank. The results of in situ hybridization techniques using TANIPCR sequences as a probe showed pericentromeric hybridization signals in all chromosomes. Nevertheless, some signals in other chromosome 
regions were also observed. We also carried out a partial genomic library using genomic DNA from $T$. nigerrimum. In all the clones in which we were able to amplify sequence TANIPCR, we have also been able to amplify a mariner-like element. This fact could indicate a relationship between the repetitive DNA and these elements, it has been as has been shown in other species of ants. The mariner elements cloned have open ORFs that could encode a protein with 345 amino acids. Genebank searches revealed that these proteins shared closed protein-sequence identity with the transposase of the Mos1 from D. mauritanica, the first mariner element described. The results of in situ hybridization techniques revealed the existence of several elements of this type in all the chromosomes.

\section{PO:06:28}

\section{Arrangement of chromosome} territories and homologous pairing in somatic nuclei of Arabidopsis thaliana

\author{
A. Pecinka ${ }^{1}$, V. Schubert ${ }^{1}$, A. Meister ${ }^{1}$, \\ G. Kreth ${ }^{2}$, M. Klatte ${ }^{1}$, M. Lysak ${ }^{1}$, J. Fuchs ${ }^{1}$ \\ and I. Schubert ${ }^{1}$ \\ ${ }^{1}$ Institute of Plant Genetics and Crop Plant \\ Research (IPK), Corrensstrasse 3, Gatersleben, \\ D-06466, Germany; ${ }^{2}$ Kirchhoff Institute for \\ Physics, University of Heidelberg, Im \\ Neuenheimer Feld 227, Heidelberg, D-69120, \\ Germany
}

Chromosome painting (CP) by fluorescence in situ hybridization (FISH) allows to study the arrangement of chromosomes in interphase nuclei. Interphase chromosomes form spatially separated chromosome territories (CTs) which may be arranged in a specific manner.

We have analysed the side-by-side arrangement of CTs and of homologous $\sim 100 \mathrm{~kb}$ chromosome segments, which might be of special interest for DNA repair via homologous recombination, in Arabidopsis thaliana root and leaf nuclei of various ploidy levels. The observations were compared to the computer model simulations for random CT arrangement. Sideby-side arrangement of all heterologous CT combinations and of homologous arm territories of chromosomes 1,3 and 5 is in accordance with the model prediction for the random arrangement. In contrast, a higher than random frequency of association was observed for the homologues of the NOR-bearing chromosomes 2 and 4, apparently due to the frequent attachment of NORs to a single nucleolus in a way mediating association of homologues. FISH with $\sim 100 \mathrm{~kb}$ segments from chromosomes 1,3 and 4 revealed homologous pairing as 7-10 times less frequent than association of homologues, not more often that expected at random and not simultaneous along the chromosomes.

Therefore, predominantly random arrangement of Arabidopsis CTs differs from that found in Drosophila (characterized by frequent somatic pairing of homologues), in spite of their similar genome size and chromosome number. Nevertheless, in up to $31.5 \%$ of Arabidopsis nuclei homologous sequences share positions which might be close enough for homologous recombination.

\section{PO:06:29}

\section{Tandem repeats inserted into two distinct loci are frequently associated with each other and with heterochromatic chromocenters in Arabidopsis thaliana interphase nuclei}

A. Pecinka ${ }^{1}$, N. Kato ${ }^{2}$, A. Meister ${ }^{1}$, A. Probst ${ }^{3}$, I. Schubert ${ }^{1}$ and E. Lam ${ }^{2}$

${ }^{1}$ Institute of Plant Genetics and Crop Plant Research (IPK), Corrensstrasse 3, Gatersleben, D-06466, Germany; ${ }^{2}$ Biotechnology Center for Agriculture and Environment, Rutgers, The State University of New Jersey, 59 Dudley Rd., New Brunswick, NJ 08901, USA; ${ }^{3}$ Laboratory of Plant Genetics, University of Geneva, Geneva 4, CH-1211, Switzerland

The green-fluorescent-protein (GFP)/lac-repressor $(\mathrm{LacI})+$ lac-operator array $(\mathrm{LacO})$ chromatin tagging system has been used in various organisms to study the dynamics and organization of the chromatin.

We have investigated the effects of the GFP/ $\mathrm{LacI}+\mathrm{LacO}$ system on the chromosome organization of transgenic Arabidopsis thaliana 2C nuclei, in which the $10 \mathrm{~kb} \mathrm{LacOs}$ were inserted 
into the top arm of chromosome 3, 4.2 $\mathrm{Mb}$ apart. We analysed GFP spots and FISH signals for LacO and transgene-flanking sequences. All GFP signals colocalized with the FISH signals, but on $\sim 17 \%$ of the tagged loci no GFP spots were detectable. The FISH analysis further revealed that $\sim 35 \%$ of the allelic tagged loci were paired in homozygous nuclei without, and $\sim 45 \%$ of loci in nuclei with GFP/LacI expression. The flanking regions paired in $\sim 5 \%$ of wild-type nuclei. About $40 \%$ of the tagged loci were found associated with heterochromatin. The regions adjacent to the transgene were 4 times less frequently associated with heterochromatic chromocenters, similar as in wild type nuclei. The results demonstrate that transgenic tandem repeats may preferentially associate with each other and with heterochromatin in Arabidopsis interphase nuclei. Expression of GFP/ LacI increases the LacO association, probably due to increased protein association.

\section{PO:06:30}

\section{Genomic variability detected using the 1 MB BAC array}

\section{E. Rajcan-Separovic ${ }^{1}$, C. Tyson ${ }^{2}$ and C. Harvard ${ }^{1}$}

${ }^{1}$ Children's and Women's Health Centre of BC and University of British Columbia, Department of Pathology, Cytogenetics, 4480 Oak Street, Vancouver, V6H 3V4, Canada; ${ }^{2}$ Cytogenetics, Southmead Hospital, Bristol, BS10 5NB, UK

The development of $1 \mathrm{Mb}$ whole genome BAC arrays offers an opportunity to screen the entire genome for subtle chromosomal changes at a resolution 10-20 higher than conventional cytogenetic analysis. The number of reports describing patients with cryptic chromosomal rearrangements detected using the array but missed by routine karyotyping is increasing and clearly shows the tremendous advantage of this method in comparison to conventional cytogenetics. However, due to the currently still limited knowledge of genetic variability at the level of a single DNA clone, usually $200 \mathrm{~kb}$ in size, the interpretation of array detected microdeletions and microduplications, particularly if they involve single clones, can be very challenging.

To achieve reliable array analysis in any population of patients a catalogue of clones showing recurrent gain or loss (polymorphic clones) is required. In the past year we have been screening the genome of patients with mental retardation for sub-microscopic chromosomal gains and losses using the commercial $1 \mathrm{Mb}$ array (Spectral Genomics). In this process we have detected clones that show recurrent change. Although some clones showed close proximity to the centromere and terminal ends of the chromosome, which are known to be rich in repeat sequences, the majority were found along the chromosomal arms. The chromosome distribution and genetic content of these clones will be discussed. Ideally, the creation of a database containing all the clones with recurrent gains and losses will facilitate the interpretation of single clone losses and gains and enhance application of array analysis in clinical diagnostic laboratories.

\section{PO:06:31}

\section{Chromosome arrangement in} interphase nuclei can be caused by the differences in scaffold-associated region (SAR) composition

\author{
A. Rodionov ${ }^{1}$, S. Galkina ${ }^{2}$ and N. Lukina ${ }^{2}$ \\ ${ }^{1}$ Botanical Institute of Russian Academy of \\ Sciences; ${ }^{2}$ Biological Institute of St. Petersburg \\ State University, Russia
}

Habermann and coauthors (2001) have shown that territories of chicken microchromosomes (MI) in cell nuclei were clustered within the center of the nucleus, while territories of macrochromosomes (MA) were located toward the nuclear periphery. In humans, small chromosomes \#\# 17, 19, 20 (but not \# 18 and Y) are located in the nuclear center, whereas the chromosomes \# 1-5 lie near nuclear envelope (Cremer et al., 2001).

Earlier, we have shown that:

1. Chicken MIs are similar to T-bands of human chromosomes, enriched in short GC-rich sequences and 'heavy' isochores (Andreozzi et al., 2002); 
2. The chicken MA gene orthologs are located in G-bands and also in T-, chromomycin-, H3-, and Alu-negative R-bands of human chromosomes, i.e., MA-gene orthologs lie on chromosomes of nuclear periphery. In contrast, the chicken MI gene orthologs are in GC-rich Giemsa-light, T-, chromomycin-, H3-, Alupositive regions of human chromosomes (Rodionov et al., 2001);

3. H3-positive R-regions of chicken MAs and MIs correspond to that of lampbrushes consisting of 'AT/GC-neutral' chromomeres. Moreover the chicken lampbrush MIs do not contain DAPI-positive chromomeres, which are the characteristic feature of MAs and correspond to theirs G-bands (Galkina et al., 2001).

As the bright chromomere fluorescence after DAPI/AMD-staining is mainly due to presence of AT-rich sequences in SARs, we suppose that 1) there are different types of SARs in T- and Gbands; 2) differentiation in SARs composition of avian MA/MI and in correspondent targets of nuclear matrix can determine the chromosome territory position in interphase nuclei.

\section{PO:06:32}

\section{D-image analysis of the topological organisation of chromatin in human lymphocytes, fibroblasts and in cancer cells of formalin-fixed, paraffin-embedded tissue sections}

S. Stein ${ }^{1}$, I. Solovei ${ }^{2}$, M. Cremer ${ }^{2}$, R. Zinner ${ }^{2}$, S. Timme ${ }^{3}$, A. Walch ${ }^{3}$ M. Werner ${ }^{3}$, M. Hausmann ${ }^{3}$ and C. Cremer ${ }^{1}$

${ }^{1}$ Kirchhoff-Institute of Physics, University of Heidelberg, Im Neuenheimer Feld 227, D-69120 Heidelberg, Germany; ${ }^{2}$ Institute of Antropology and Human Genetics, University of Munich, Richard-Wagner-Str. 10, D-80333 Munich, Germany; ${ }^{3}$ Institute of Pathology, University Hospital, Albertstr. 19, D-79104 Freiburg, Germany

The higher order chromatin architecture is not completely understood. FISH techniques and high resolution microscopy contribute to its further elucidation. Therefore, novel tools are required to analyse $3 \mathrm{D}$ digital images and to determine appropriate parameters to quantify the architecture and organisation. A software package was developed specifically for FISH labelled cell nuclei and 3D-fluorescence microscopy. For processing a huge package of $3 \mathrm{D}$-image data an user-friendly shell script has been developed, which makes it possible to process a lot of 3Dimages in a very short time. To ensure that measurements of the parameters are threshold independent, the whole intensity range is analysed. The segmentation of the FISH signals is based on a local 26 relationship. The criterion for a voxel to be inside a local 26 neighbourhood of another voxel is, that the voxels must be connected at their sides, edges or corners. Because of the intensity dependent parameters it is feasible to get information about the topology and the interior organisation of chromatin, e.g. the radial position in the cell nucleus, relative distances to other marker, or the shape of the labelled site. The software was successful tested and applied on territories of chromosome \#18, \#19 and \#X in human lymphocytes and fibroblasts, on territories of chromosome \#8 in nuclei of pancreatic tumour cells in formalin-fixed, paraffin-embedded tissue sections, and on H3K9 marked heterochromatin. The results indicate the possibility to characterise differences in the architecture and organisation of the FISH labelled objects in order to distinguish normal and aberrant cell nuclei.

\section{Reproduction Symposium}

\section{L47}

\section{Investigation of aneuploidy} mechanisms by FISH and CGH analysis of oocytes and polar bodies

\author{
J. Delhanty ${ }^{1}$, E. Fragouli ${ }^{1}$, D. Wells ${ }^{3}$, \\ C. Conn ${ }^{1}$, K. Whalley ${ }^{2}$, J. Mills ${ }^{2}$ and \\ M. Faed ${ }^{2}$
}

${ }^{1}$ Department of Obstetrics \& Gynaecology, UCL, 86-96 Chenies Mews, London, WC1E 6HX, UK; ${ }^{2}$ Ninewells Hospital Dundee, Dundee, DD1 9SY, Scotland, UK; ${ }^{3}$ St Barnabas Medical Center, West Orange, NJ 07052, USA 
Molecular cytogenetic investigations of cleavage stage embryos have revealed anomalies affecting all sizes of chromosomes. The aim was to investigate the variety of anomalies arising during maternal meiosis I by analysis of unfertilised oocytes and polar bodies to gain insight into aneuploidy mechanisms.

Sequential FISH analysis was carried out with specific probes derived from eight chromosomes, representing all sizes. Only imbalance due to a gain of a whole chromosome or chromatid, represented by extra signals, was counted, to avoid artefact. In a separate approach, DNA was extracted from oocytes and polar bodies for use in CGH analysis.

Data were obtained from FISH analysis on 236 eggs from 124 patients of average age 32.5 years (range 22 to 44 ). Ten patients (average 32.6 years) had abnormal eggs. The abnormality rate for oocytes and for polar bodies was close to $4 \%$ for each. Fourteen hyperploidies were found, seven involving additional single chromatids. The abnormalities affected chromosomes 13,16,18, 21 and $\mathrm{X}$ but not chromosomes 1,9 , or 12 . CGH results are available for a small number of samples to date. Anomalies have been found for a wider range of chromosomes but patient specific factors are involved.

The data provide evidence for several aneuploidy mechanisms including classical nondisjunction of whole univalents; pre-division of chromatids prior to anaphase I, leading to imbalance detected at metaphase II; germinal mosaicism for a trisomic cell line and preferential involvement of the smaller chromosomes.

\section{L48}

\section{OLIGO meiotic maturation arrest and recombination deficiency causative of male factor infertility}

\section{Hultén ${ }^{1}$}

${ }^{1}$ Department of Biological Sciences, University of
Warwick, Coventry CV47AL, UK

Infertility affects around 1 in 6 couples with more than $50 \%$ attributed to factors leading to disturbance of spermatogenesis. Attention has focussed on chromosome abnormalities (such as XXY, XYY, translocations, inversions and insertions) and DNA mutations (such as $\mathrm{Yq}$ deletions) diagnosed by standard karyotyping and molecular investigations using somatic tissue samples (usually blood samples). By this approach, however, the underlying cause remains unknown in an outstanding majority of infertile men with impaired spermatogenesis. On the other hand, direct meiotic investigations using testicular biopsy samples, revealed that around 1 in 5 such men with severe oligo- or azoospermia show a delay in meiosis1 maturation during the prophase stages leptotene and zygotene and a partial arrest at the subsequent pachytene stage in addition drastic reduction in chisma frequency at metaphase1 and increased aneuploidy at metaphase2. There are also indications that milder variants may affect men with less severe disturbance of spermatogenesis. This condition, which we have termed OLIGO, is characterised by increased parental consanguinity and familiar occurrence of reduced fertility, and is suggested to represent the first recognised (recessive) meiotic mutation in humans. It seems likely OLIGO contributes substantially to the increased rate of aneuploid offspring by ICSI; hopefully recent scientific advances (including identification of recombination foci by MLH1 immunofluorescence) and increased knowledge on meiotic mutants in mice will soon allow recognition of the genetic mutation per se, facilitating adequate diagnosis and genetic counselling in the first instance in male factor infertility.

\section{L49}

Segregation study of Robertsonian and reciprocal translocations in sperm and pre-implantation embryos

E. Van assche ${ }^{1}$, G. Ogur ${ }^{1}$, C. Staessen ${ }^{2}$, M. Bonduelle ${ }^{1}$, A. Michiels ${ }^{1}$, G. Verheyen ${ }^{2}$, K. Keymolen ${ }^{1}$, P. Devroey ${ }^{2}$,

A. Van Steirteghem ${ }^{2}$ and I. Liebaers ${ }^{1}$

${ }^{1}$ Center for Medical Genetics, University Hospital, Dutch-speaking Free University of Brussels, Laarbeeklaan 101, 1090 Brussels, Belgium; ${ }^{2}$ Center for Reproductive Medicine, University Hospital, Dutch-speaking Free University of Brussels, Laarbeeklaan 101, 1090 Brussels, Belgium. 
In the present study the meiotic segregation behaviour in spermatozoa, from 14 Robertsonian (Rob) and 20 reciprocal (Rcp) translocation carriers was determined by two- or three- colour FISH, with selected centromeric and/or regionspecific and/or sub-telomeric probes according to the translocation type. Pre-implantation genetic diagnosis (PGD) was performed in 6/14 Rob carriers (14 cycles) and in 7/20 Rcp carriers (16 cycles). The different types of meiotic products (alternate, adjacent 1 and 2 and 3:1 segregation) were identified and compared in sperm and preimplantation embryos. In the different Rob carriers, a high rate of balanced spermatozoa (range: $76 \%-89.4 \%$ ) and consequently a relatively low rate of unbalanced spermatozoa (range: 10.6\%$24 \%$ ), was observed. In contrast, the incidence of unbalanced sperm in the different Rcp carriers was highly variable from one translocation to another (range: $10.4 \%-79.6 \%$ ), but for $19 / 20$ carriers the rate was often higher than $50 \%$. During PGD, $53.4 \%$ of the analysed embryos seemed to be normal in the Rob carriers and only $21.5 \%$ in the Rcp carriers. Considering the meiotic behaviour of the different translocations, comparable segregation patterns were observed in sperm and embryos. In all cases, the frequency of imbalances in sperm and embryos was above the average rate of imbalance observed at term or in prenatal diagnosis demonstrating the presence of an efficient selection against unbalanced embryos/foetuses further in pregnancy.

\section{L50}

Evidence for a significant reduction in sperm aneuploidy levels coincident with treatment by traditional Chinese herbal medicine and for endocrine and anti-oxidant activity of the therapeutic herbs

H. Tempest ${ }^{1}$, D. Christopikou ${ }^{1}$, M. Dalakiouridou ${ }^{1}$, X. Zhai ${ }^{2}$, S. Homa ${ }^{2}$ and D. Griffin ${ }^{1}$

${ }^{1}$ Cell and Chromosome Biology Group, Department of Biological Sciences, Brunel University, Uxbridge, UB8 3PH. UK;

${ }^{2} 112$ Harley Street, London, UK
The incidence of aneuploidy in sperm of men with male factor infertility is one of the most contentious issues in reproductive medicine. In particular, the risk of ICSI treatment of these men unwittingly giving rise to aneuploid offspring has raised concerns in the scientific and popular press. To the best of our knowledge however there are no reports of treatment regimes purporting to successfully reduce the incidence of sperm aneuploidy. Through the use of three colour FISH, we have evidence of a significant reduction in sperm aneuploidy frequencies for chromosomes $21, \mathrm{X}$ and $\mathrm{Y}$ in a cohort of six patients undergoing TCM treatment. In each patient initial aneuploidy frequencies were significantly higher than controls for at least one of the investigated chromosomes, however coincident with TCM treatment, aneuploidy levels fell significantly. In addition, experiments were undertaken to determine whether individual herbs and/or the combinations used for treatment exhibited biological activity. All herbs were tested for anti-oxidant activity and endocrine properties (including oestrogenic, antioestrogenic, androgenic and anti-androgenic activity). Analysis of the biological activity of the herbs revealed that the majority of the individual herbs and all of herb prescriptions tested produced both anti-oxidant activity and antioestrogenic activity. These results provide evidence of a reduction in aneuploidy frequencies coincident with TCM treatment and suggestions of possible biological pathways through which they may be acting. We propose that placebo controlled clinical trials should be considered using this treatment with a view to ultimately providing an alternative or an adjunct to ICSI.

\section{L51}

\section{Cytogenetic analysis of human blastocysts}

H. Clouston ${ }^{2}$, A. Murdoch ${ }^{3}$ and J. Wolstenholme ${ }^{1}$

${ }^{1}$ Department of Cytogenetics, Institute of Human Genetics, International Centre for Life, Newcastle upon Tyne, UK; ${ }^{2}$ (current address of HJC) North Trent Cytogenetics Service, Sheffield Childrenâs 
Hospital, Sheffield, UK; ${ }^{3}$ Newcastle Fertility Centre, International Centre for Life, Newcastle upon Tyne, UK

The blastocyst is a relatively understudied stage of human development, but one that appears key in the aetiology of many abnormal karyotypes. In the largest study so far, 671 spare IVF/ICSI blastocysts, were subjected to thymidine-based synchronisation of cell division and acetic acid disaggregation[1,2]. Metaphases were obtained from 477 blastocysts. Of these, 248 blastocysts were successfully karyotyped, and partial analysis of a further 199 blastocysts generated ploidy information. Almost 30\% of blastocysts demonstrated diploid:tetraploid mosaicism.

Among fully karyotyped blastocysts, two $(0.8 \%)$ were triploid and one $(0.4 \%)$ was fully tetraploid. Nine blastocysts $(3.6 \%)$ had aneuploidies confirmed in multiple cells, and four $(1.6 \%)$ were classified as trisomic based on a single cell analysis. Two blastocysts $(0.8 \%)$ were confirmed mosaic trisomies and a further twelve $(4.8 \%)$ were possible mosaic trisomies (a single trisomic cell was found alongside normal cells). Sequential FISH techniques enabled further characterisation of abnormal findings, in particular the clarification of ambiguous results and the investigation of mosaicism in interphase nuclei.

Comparison with abnormality rates at earlier and later stages of gestation allows two main conclusions to be drawn. Firstly, it appears that significant selection against abnormal embryos occurs prior to blastocyst development. Secondly, the general range and incidence of most main groups of chromosome abnormality observed in first trimester pregnancies appear to be in place by the blastocyst stage.

1. Clouston et al, (1997) Hum Genet 101: 30-36

2. Clouston et al, (2002) Prenat Diagn 22: 1143-52

\section{L52}

Full karyotyping of human preimplantation embryos using comparative genomic hybridisation (CGH)

\section{Wilton ${ }^{1}$}

${ }^{1}$ Genetic and Molecular Research Laboratory, Melbourne IVF, Australia

FISH studies have demonstrated a high frequency of aneuploidy in early embryos from in vitro fertilisation (IVF) patients. Most of these aneuploidies are lethal and would prevent the embryos from implanting and/or developing to term. IVF clinics perform preimplantation genetic diagnosis for aneuploidy screening (PGDAS) using FISH for chromosomes X, Y, 13, 16, 18,21 and 22 to test single cells biopsied from embryos. This allows selection of embryos most likely to be viable for transfer to the patient. An obvious limitation is that FISH is restricted to identification of a few chromosomes and the full extent of aneuploidy in early embryos remains largely unknown. We have developed single cell $\mathrm{CGH}$ and successfully used it to fully karyotype cells from embryos for PGD-AS (Wilton et al., Fertil Steril 2003 80:860-8). Consequently, we have data describing chromosome abnormalities in more that 300 aneuploid cells from approximately 120 3-day old ( 8-cell) human embryos. This includes multiple cells from more than 60 embryos.

Every chromosome was involved in aneuploidy. Approximately $40 \%$ of cells had a single aneuploidy, $10 \%$ had double aneuploidy and about $50 \%$ showed extensive aneuploidy of $>3$ chromosomes. Analysis of multiple cells from the same embryo showed that approximately $25 \%$ of embryos had consistent aneuploidy in each cell, which was presumed to be meiotic in origin. About $75 \%$ of embryos had different aneuploidies in different cells, which probably occurred postzygotically. Many embryos had aneuploidies that would not have been identified using FISH highlighting the benefit of full karyotyping for PGD-AS.

\section{L53 \\ Deletions and duplications between palindromes $P 5$ and $P 1$ in subfertile men}

K. Writzl ${ }^{1}$, B. Zorn ${ }^{2}$ and B. Peterlin ${ }^{1}$ 
${ }^{1}$ Division of Medical Genetics, Department of Obstetrics and Gynecology, UMC Ljubljana; Ljubljana, Slovenia; ${ }^{2}$ Andrology Centre, Department of Obstetrics and Gynecology, UMC Ljubljana; Ljubljana, Slovenia

Microdeletions of the long arm of human Y chromosome represent an important cause of male infertility. Analysis of large deletions of the Y chromosome [including azoospermia factor $\mathrm{b}$ (AZFb) and AZFc loci] has shown that large palindromes on Yq (named P1-P5) serve as substrates for non-allelic homologous recombination. This form of male infertility can thus be classified as a genomic disorder. The reciprocal products of these deletion events, duplications, which are expected to occur at the same frequency as deletions, have not been reported to date.

Therefore, our objective in this study was to determine whether the reciprocal duplications between palindromes $\mathrm{P} 5$ and $\mathrm{P} 1$ occur in subfertile men.

Ninety patients with male idiopathic subfertility (44 azoospermics and 46 oligozoospermics) were analysed for deletion/duplication of the sY125 locus using real time PCR. Two azoospermic men had deletion of the sY125 locus (also detected by PCR screening of microdeletions of the Y chromosome). No duplication of the sY125 locus was detected.

Duplications of the region between palindromes P5 and P1 don't seem to be a significant risk factor for spermatogenic failure.

\section{L54}

\section{Age-related aneuploidy in unfertilised oocytes: Comparison of CGH and FISH analysis}

\section{Gutiérrez-Mateo ${ }^{1}$, D. Wells ${ }^{2}$, J. Benet ${ }^{1}$, P. Colls ${ }^{3}$, J. Sánchez-Garcia ${ }^{1}$, M. Bermúdez ${ }^{2}$, S. Munné ${ }^{2}$ and J. Navarro ${ }^{1}$}

${ }^{1}$ Departament de Biologia Cellular, Fisiologia i Immunologia, Unitat de Medicina, Universitat Autonoma de Barcelona, E-08193 Bellaterra, Spain; ${ }^{2}$ The Institute for Reproductive Medicine and Science, St. Barnabas Medical Center, 101 Old Short Hills Road, Suite 501, West Orange, NJ 07052, USA; ${ }^{3}$ Reprogenetics, Hoboken, New Jersey, USA
The increase in aneuploidy with maternal age leads to an increased risk of producing aneuploid offspring and spontaneous abortions as well as a decrease in implantation rates. To determine the mechanisms that produce aneuploidy in oocytes and establish which chromosomes are more prone to aneuploidy, 33 unfertilized oocytes from women 35 years old (mean, 38.2) were analysed. The whole chromosome complement of the first polar body (1PB) was analyzed by comparative genomic hybridisation $(\mathrm{CGH})$, while the corresponding MII oocyte was analyzed by fluorescent in situ hybridisation (FISH) to confirm the results. Matched CGHFISH results were obtained in twenty-nine doublets 1PB-MII, twenty-five $(86.2 \%)$ showing reciprocal results. The aneuploidy rate was $60.6 \%$. FISH analysis revealed that as much as two thirds of the aneuploid events were chromatid abnormalities, while only one third were due to non-disjunction of bivalents. Interestingly, the chromosomes more frequently involved in aneuploidy were, in order, chromosomes 16, 1, 4 and 15. Classifying the chromosomes as large (chromosomes 1-12 and X) or small (chromosomes 13-22), small chromosomes were more prone to aneuploidy (Chi2 test, $\mathrm{P}<0.025$ ). Four out of twenty aneuploid doublets $(20 \%)$ would have been missdiagnosed as normal using FISH with 9 chromosomes probes $(1,13,15,16,17,18,21$, 22 and $\mathrm{X}$ ). In this study, the combination of two different techniques, CGH and FISH, for the study of $1 \mathrm{~PB}$ and MII allowed the identification and confirmation of any numerical chromosome abnormality, as well as helping to determine the mechanisms involved in the genesis of maternal aneuploidy.

\section{PO:06:33}

\section{Chromosomal anomalies in referred patients with male infertility}

\author{
S. Bheemineni ${ }^{1}$, J. Solanki ${ }^{2}$, R. Uppala ${ }^{1}$ \\ and R. Uppala ${ }^{1}$ \\ ${ }^{1}$ Green Cross blood bank \& Genetic Research \\ Centre, Anil Kunj, Paldi, Ahmedabad-380014, \\ India; ${ }^{2}$ Department of Animal Genetics and \\ Breeding, Veterinary College, Gujarat Agriculture \\ University, Anand-388001, India
}


Infertility is a well-known clinical manifestation of various ethical factors and is defined as an inability to conceive after 12 months of unprotected intercourse. The prevalence of infertility couples ranges between 2 and $16 \%$, with $50 \%$ of cases due to male infertility. Many cases of male infertility are due to identifiable severe defects in sperm production, which are classified as azoospermia, oligospermia, abnormal motility or morphology and some are idiopathic. Several factors are associated with infertility few of them included infection, immunological factors, anatomic abnormalities, environmental etiologies, endocrinologic dysfunctions and some are influenced by genetic factors. We have analyzed 80 male infertility patients for possible chromosomal anomalies. After the detailed family history and pedigree analysis, routine PHA stimulated peripheral blood cultures were set up and karyotyping was done after GTG banding and other banding techniques were performed as required. A high incidence of $20 \%(n=16)$ of chromosomal abnormalities was observed among 80 infertility patients referred (i.e. both structural and numerical). Those included $5 \%$ with balanced reciprocal translocations $(\mathrm{N}=4), 3.75 \%$ with sex reversals $(\mathrm{n}=3)$ and $12.5 \%$ with gonosomal aneuploidies/mosaicisms $(n=9)$. These data suggests that patients with history of infertility should be investigated cytogenetically. DNA microsatellite and FISH analysis are in progress in few patients found with rare chromosomal translocations and also $\mathrm{Y}$ chromosome microdeletions analysis.

\section{PO:06:34}

\section{Comparative genomic hybridisation analysis of first polar bodies and metaphase II oocytes}

C. Gutiérrez-Mateo ${ }^{1}$, D. Wells ${ }^{2}$, J. Benet ${ }^{1}$, J. Sánchez-Garcia ${ }^{1}$, M. Bermúdez ${ }^{2}$, I. Belil ${ }^{3}$, J. Egozcue ${ }^{1}$, S. Munné ${ }^{2}$ and J. Navarro ${ }^{1}$

${ }^{1}$ Departament de Biologia Cellular, Fisiologia i Immunologia, Unitat de Medicina, Universitat Autonoma de Barcelona, E-08193 Bellaterra, Spain; ${ }^{2}$ The Institute for Reproductive Medicine and Science, St. Barnabas Medical Center, West
Orange, NJ 07052, USA; ${ }^{3}$ Servei de Medicina de la Reproduccion, Institut Universitari Dexeus, Barcelona, Spain

Preimplantation Genetic Diagnosis (PGD) of aneuploidies has achieved a reduction of spontaneous abortions and increased implantation and pregnancy rates in certain groups of patients. The technique most commonly used has been fluorescent in situ hybridisation (FISH), but the number of chromosomes that can be analysed is a clear limitation of this method. We evaluated the limitations and reliability of using comparative genomic hybridisation (CGH) to detect the whole set of chromosomes, as an alternative to PGD using FISH. Fifty single cells were analysed, corresponding to 25 metaphase II oocytes (MII) and their complementary first polar bodies (1PB). The aneuploidy rate found was $48 \%$. Dividing the data into two maternal age groups $(<37$ and $\geq 37$ years old), more chromosome abnormalities were found in the older group ( $23 \%$ and $75 \%$, respectively, $\mathrm{P}<0.02)$. Six out of twenty-five doublets 1PB-MII (24\%) did not display reciprocal results, as one cell showed one or two missing chromosomes while their sibling cell showed a normal profile for those chromosomes. About $33 \%$ of the doublets 1PB-MII diagnosed as aneuploid by CGH would have been incorrectly diagnosed as normal if FISH with 9 chromosome probes had been used. In this study we demonstrate the reliability of $1 \mathrm{~PB}$ analysis by CGH to detect almost any chromosome abnormality in oocytes in a time frame compatible with regular in vitro fertilization. The application of CGH in PGD of aneuploidy to select chromosomally normal oocytes could help to increase implantation and pregnancy rates of patients undergoing in vitro fertilization treatment.

\section{PO:06:35}

\section{The role of chromosomal aberrations to the no-fertilization in oocytes after FIVET or ICSI techniques}

\author{
G. Nannetti ${ }^{1}$, S. Bonifacio ${ }^{1}$, E. Lisi1, \\ S. Carniani ${ }^{1}$, N. Nadalin ${ }^{1}$ and F. Torricelli ${ }^{1}$ \\ ${ }^{1}$ UO Citogenetica e Genetica, AO Careggi, \\ Firenze Italy
}


Techniques such as FIVET or ICSI have allowed a practical treatment of infertility. The purpose of our study was to detect numerical chromosomal aberrations in oocytes subjected to ICSI or FIVET but not outcomed in embryos.

The cytogenetic results obtained by FISH allowed us to evaluate chromosomal anomalies that could explain the failure of fertilization.

The study has been structured on the study of 54 oocytes: 39 of them had not presented fertilization after FIVET and 15 after ICSI.

Oocytes were hybridized with locus specific probe for chromosome 21 and alpha satellite probes for chromosomes $\mathrm{X}$ and $\mathrm{Y}$ after a pretreatment with $\mathrm{HCl}$ and Tween 20 solution. Y chromosome probe was used to detect sperm DNA.

About $95 \%$ of the analyzed oocytes exhibited discernible FISH signal.

For metaphase II ICSI oocytes, with or without pronuclear formation, and for the FIVET oocytes, our objective was to determine their chromosomal status.

Our results confirms the utility of FISH technique for determining the relatively low fertilization rates.

PO:06:36

\section{Complex balanced chromosomal rearrangement explains reproductive difficulties (recurrent abortions) in a healthy female: a case report}

\section{A. Simons ${ }^{1}$, H. Mielooo ${ }^{1}$, F. Van $\operatorname{Erp}^{1}$ and D. Smeets ${ }^{1}$}

${ }^{1}$ Dept. of Human Genetics, Division of Cytogenetics, UMCN St. Radboud Nijmegen, PO box 9101, 6500 HB Nijmegen, The Netherlands

Routine cytogenetic analysis was performed on cultured peripheral lymphocytes of a woman and her partner, because of the occurrence of two subsequent spontaneous abortions. While her partner showed a normal male $(46, \mathrm{XY})$ karyotype, a complex balanced chromosomal rearrangement involving chromosomes 7 (q31.2), 12 (q22) and 13 (q21.2 and q22.3) was found to be present in the healthy woman. Regular GTGbanding and additional FISH analyses showed two cytogenetic rearrangements which occurred simultaneously: a reciprocal translocation between the chromosomes 12 and 13 and an insertion of a part of the derivative translocation chromosome 13 into chromosome 7. The complexity of this 'three-way'-rearrangement gives a high risk for unbalanced karyotypes in offspring (ranging from spontaneous abortions to unbalanced/affected life-born) and explains the experienced repetitive abortions in this 36 yearold woman.

Here, we present the results of the (molecular) cytogenetic analyses, propose a mechanism of occurrence of the rearrangements and discuss the clinical consequences of the various unbalanced karyotypes.

\section{Comparative Genomics / Chromosome Evolution Symposium}

\section{L55}

\section{Contribution of cross-species chromosome painting to comparative genomics}

\section{Ferguson-Smith ${ }^{1}$}

${ }^{1}$ Cambridge University Centre for Veterinary Science, UK

Comparison between human and representative species of all extant mammalian orders using chromosome painting reveals the remarkable conservation of genomes throughout evolution. Large regions of chromosome, including whole chromosomes and chromosome arms, are often shared between species. Differences in chromosome number and structure result from rare events of illegitimate meiotic recombination leading to chromosome fusion, fission and other inter- and intra-chromosomal rearrangements. Characteristically, these events occur at sites of DNA duplication. They have been used in constructing phylogenetic trees and in the prediction of the likely ancestral mammalian karyotype. Recent studies have used chromosomal painting to help explain the occasional fertility of mules, to interpret the complex meiotic multivalent 
produced by the ten unpaired chromosomes of the male platypus and to identify the homomorphic sex chromosomes of the crocodile that is homologous to the avian $\mathrm{Z}$ chromosome. Simple comparative maps have been made between mapped and unmapped species, and DNA microarray painting is being used to characterise evolutionary breakpoints. The current challenge is to overcome the limitations of chromosome painting between mammalian species and those of birds, reptiles, marsupials and monotremes.

\section{L56}

\section{The chicken genome: A resource for chicken and evolutionary biology}

\section{Burt ${ }^{1}$}

${ }^{1}$ Roslin Institute, Edinburgh, Scotland, UK

In this paper we review progress in chicken genomics and introduce ChickNET: an international organisation devoted to the analysis and application of genomics to avian biology. The chicken genome project has its roots in a decade of map building by genetic and physical mapping methods. Chicken genetic markers for map building have generally depended on labour intensive screening procedures. In recent years this has all changed with the availability of $\sim 500 \mathrm{~K}$ EST sequences, a draft sequence of the entire chicken genome and a map of over 1 million SNPs. Clearly, the future for the chicken genome and avian research is an exciting one. Through the integration of these resources, it will be possible to solve challenging scientific questions exploiting the power of a chicken model . the aim of ChickNET is to drive this vision. In this talk I wil review progress and some of the conclusions from the analysis of this genome sequence in terms of its evolution and comparison with other genomes.

\footnotetext{
Acknowledgements

I am grateful to my colleagues and many collaborators who continue to contribute to the development of chicken genome (see ChickNET www site) and acknowledge the financial
}

support of the Botechnology and Biological Sciences Research Council (BBSRC)

L57

\section{The moving landscape of comparative genomics in mammals}

\author{
S. O'Brien ${ }^{1}$ \\ ${ }^{1}$ NCI-Frederick, MD, USA
}

The full genome sequence of human, mouse, dog and rat genomes combined with dense physical genetic maps have been complemented by precise gene homologue alignment of dense physical genetic maps of livestock, companion animals and additional mammal species. Comparative genetic assessment expands the utility of these maps in gene discovery, in functional genomics, and in tracking the evolutionary forces that sculptured the genome organization of modern mammalian species.

\section{L58}

\section{Primate chromosomes: phylogenetic landmarks, evolutionary breakpoints and comparative nuclear architecture}

\author{
S. Müller ${ }^{1}$ \\ ${ }^{1}$ Department Biology II, Human Genetics, \\ Ludwig-Maximilians University, Munich, \\ Germany
}

To date, over 80 mammalian species were investigated by multi-directional chromosome painting, among them approximately 60 primates from every major phylogenetic lineage. A more detailed comparative analysis employing chromosome bar codes allowed the assignment of subregional homologies between various higher primate species. BAC and YAC contigs have been used for the establishment of high-resolution comparative maps and a detailed analysis of evolutionary breakpoints in several human homologous chromosomes. The resulting chromosomal homology maps between numerous primate species allowed the detailed reconstruction of evolutionary chromosome rearrangements. It has also become 
possible to draw increasingly accurate conclusions about the direction of these changes and to establish chromosomal phylogenies. Moreover, this knowledge has been prerequisite to address open questions concerning evolutionary conserved motives of higher order chromatin arrangements and their possible consequences on the nature and direction of chromosome evolution.

The presentation provides an overview of technological developments in primate comparative cytogenetics and gives a reconstruction of the major landmarks in primate karyotype evolution. Further, recent results obtained from the analysis of evolutionary chromosomal breakpoints and from comparative studies of primate nuclear architecture are summarized

\section{L59}

\section{Pathways of rapid karyotype evolution in Hypochaeris (Asteraceae), with special emphasis on polyploids}

\author{
H. Schneeweiss ${ }^{1}$, T. Stuessy ${ }^{1}$, K. \\ Tremetsberger $^{1}$, S. Siljak-Yakovlev ${ }^{2}$ and \\ J. Parker ${ }^{3}$
}

${ }^{1}$ Department of Higher Plant Systematics and Evolution, Institute of Botany, University of Vienna, Rennweg 14, A-1030 Vienna, Austria; ${ }^{2}$ Département Evolution \& Systématique, UPRESA CNRS 8079, Université de Paris-Sud, Orsay Cedex, France; ${ }^{3}$ Botanic Garden, Cambridge University, Cambridge CB2 IJF, UK

The genus Hypochaeris (Compositae) has a disjunct distribution with approximately 10 species in Eurasia and NW Africa and 50 species in South America. The genus arrived in South America from the Old World within the past 3-5 my, and evolved there rapidly within 1-2 my. The European species possess symmetrical karyotypes with $\mathrm{x}=3,4,5$, and 6 , whereas the South American species have asymmetric karyotypes with $\mathrm{x}=4$. Chromosome number and morphology are similar in all South American species, but molecular cytogenetic analyses of more than 20 species reveal differentiation of karyotypes based on $18 \mathrm{~S}-25 \mathrm{~S}$ and $5 \mathrm{~S}$ rDNA localization and allow discrimination of five karyotypic groups. A model of karyotype evolution within the South American groups is proposed, based primarily on number and position of $18 \mathrm{~S}$ 25S rDNA translocations. Cytogenetic analysis of the North African species, Hypochaeris angustifolia, reveals the same basic chromosome number as South American taxa $(\mathrm{n}=4)$, and thereby serves to connect Old World (e.g., H. maculata) and New World taxa. Polyploids (all tetraploids) are more common among South American Hypochaeris than previously reported and are often present within diploid populations (cytotype mixture), suggesting autopolyploid origins. Tetraploids often differ karyotypically from their diploid relatives, some representing unique karyotypes, and appear to undergo more genome rearrangements than diploids, as judged by rDNA sequence localization. Elimination and translocation of 18S-25S-rDNA loci are pronounced trends.

\section{L60}

\section{Precise characterization of pericentric inversions that distinguish human and chimpanzee genomes}

J. Szamalek ${ }^{1}$, V. Goidts ${ }^{1}$, C. Sandig ${ }^{1}$, N. Chuzhanova ${ }^{2}$, S. Tänzer ${ }^{3}$, S. Müller ${ }^{4}$, M. Platzer ${ }^{3}$, D. Cooper ${ }^{5}$, H. Hameister ${ }^{1}$ and H. Kehrer-Sawatzki ${ }^{1}$

${ }^{1}$ Department of Human Genetics, University of Ulm, Ulm, Germany; ${ }^{2}$ Department of Computer Science, Cardiff University, Cardiff, UK;

${ }^{3}$ Department of Genome Analysis, Institute of Molecular Biotechnology, Jena, Germany;

${ }^{4}$ Institute of Anthropology and Human Genetics, University of Munich

(Ludwig-Maximillians-University), Munich;

${ }^{5}$ Institute of Medical Genetics, University of Wales College of Medicine, Cardiff, UK

Although human and chimpanzee karyotypes are similar, some important differences are apparent. These are the fusion that gave rise to human chromosome 2, variations in the amount of constitutive heterochromatin and nine pericentric inversions. These inversions have attracted considerable interest, since they may have influenced the parapatric speciation of early hominids and chimpanzees. Breakpoint analysis of the 
pericentric inversions is a prerequisite to determine their biological consequences and to explore the mechanisms underlying primate chromosome evolution. We isolated breakpoint-spanning BACs from the human and the chimpanzee genomes and identified the breakpoints. Comparative sequence analysis revealed that the inversion breakpoints of chromosomes homologous to human chromosomes 4, 5, 9, 16, and 17 occurred in regions rich in high-copy repeats. The humanspecific inversion of chromosome 18 appears to have been mediated by segmental duplications of $19-\mathrm{kb}$. By contrast, the inversion of the chimpanzee chromosome homologous to HSA12 is associated with two duplications of $86-\mathrm{kb}$ and $23-\mathrm{kb}$ both of which occurred in the chimpanzee lineage. None of the pericentric inversions disrupts the protein-coding region of a gene.

Interestingly, seven of the nine pericentric inversions are chimpanzee-specific, whereas humans have retained the ancestral, unrearranged state of the respective chromosomes. Thus, the human karyotype is closer to an ancestral primate karyotype than that of the chimpanzee. FISH with breakpoint-spanning BACs indicated that bonobo (Pan paniscus) and Pan troglodytes share the same inversions. Therefore, their fixation predated the separation of these two lineages and would have occurred in the time interval from 5-6 to 1.8-Mya.

\section{L61}

\section{Unusual features of the platypus and echidna karyotypes revealed by cross-species chromosome painting}

W. Rens ${ }^{1}$, F. Grutzner' ${ }^{2}$, P. O'Brien ${ }^{1}$, H. Fairclough ${ }^{1}$, J. Graves ${ }^{2}$ and M. Ferguson-Smith ${ }^{1}$

\footnotetext{
${ }^{1}$ Molecular Cytogenetics Laboratory, Centre for Veterinary Science, Department of Clinical Veterinary Medicine, University of Cambridge, Madingley Road, Cambridge, UK; ${ }^{2}$ Research School of Biological Sciences, Australian National University, Canberra, Australia
}

We have determined the identity of the unpaired chromosomes that form a multivalent chain in male meiotic cells of the duck-billed platypus and the short-beaked echidna by chromosome painting of mitotic cells. The use of this technique for identification was necessary as many of the monotreme chromosomes are small metacentrics and the order and relationship of the unpaired chromosomes require the identification of small homologous pairing regions. The existence of a large set of unpaired chromosomes, which have to segregate in meiosis, raises questions about the evolution of the sexchromosome system in monotremes.

The platypus and echidna are the only extant monotreme species. The Australian platypus (Ornithorhynchus anatinus) belongs to the Ornithorhynchidae family, whilst the short-beaked echidna (Tachyglossus aculeatus) and the long beaked echidna (Zaglossus bruijnii) belongs to the Tachyglossidae family.

In the platypus unpaired chromosomes are present in the male only (data on female echidna is as yet not available). The females have two copies of the odd-numbers in the male multivalent chain, i.e. element numbers 1,3,5,7, and 9; the even numbers are absent. This requires an alternating pattern of chromosome segregation in male meiosis. Large sections of the odd numbered chromosomes do not have homologous regions with their even numbered neighbours. Thus these regions are present as one copy in male and two copies in female and behave like 'X-chromosome regions'. We have used cross species painting to show the degree of chromosome conservation between these two extant monotreme species.

\section{L62}

\section{Evolution of genes located on the Y-chromosome in the cat family Felidae}

\section{J. Pecon-Slattery' ${ }^{1}$ V. King ${ }^{2}$, W. Murphy', A. Pearks Wilkerson ${ }^{1}$ and S. O'Brien ${ }^{1}$}

${ }^{1}$ Laboratory of Genomic Diversity, National Cancer Institute-Frederick, Frederick MD 21702, USA; ${ }^{2}$ The Center for the Advancement of Genomics, Rockville MD, 20850 USA

The recent radiation of the cat family Felidae offers a unique opportunity to examine patterns of evolution within Y-chromosome genes. Genomic segments from single copy $\mathrm{X}-\mathrm{Y}$ homologues 
SMCY, UBE1Y and ZFY (3604 bp) were amplified in 36 species of cat. These genes are located within the X-degenerate region of the NRY and are thought to be 'fossils' that ceased conventional recombination with the X-chromosome early within the placental mammal evolution 65-104 MYA. The pattern and tempo of evolution at these three genes is significant in light of the recent, rapid evolution of the family over approximately $12 \mathrm{MY}$ and provides exceptional support for each of the eight recognized felid lineages as well as clear diagnostic substitutions identifying nearly all species. Bootstrap support and Bayesian posterior probabilities are uniformly high for each lineage. A similar analysis of the male-determining gene SRY and its adjacent genomic flanks (2957 bp) suggests this reproductively important gene to be tightly linked with speciation. Adaptive evolution of SRY is assessed by codon analyses. No specific sites are under selection, but an abundance of $\mathrm{w}>1$ ( $\mathrm{w}=\mathrm{Dn}: \mathrm{Ds})$ among all branches within the SRY tree suggests most speciation events are marked by nonsynonymous changes. The precise phylogenetic results obtained using the well-characterized Felidae as a species tree suggest genes within the NRY are powerful markers of evolution within species.

\section{L63}

Multiple chromosomal heteromorphisms in Tympanoctomys barrerae, $2 \mathrm{n}=102$ (Caviomorpha, Octodontidae): polyploidy or polymorphism?

\section{Bidau', M. Gallardo ${ }^{2}$, C. Lanzone ${ }^{3}$, R. Stanyon ${ }^{4}$ and J. Santos ${ }^{5}$}

${ }^{1}$ Universidade do Estado do Rio de Janeiro, Brazil; ${ }^{2}$ Universidad Austral de Chile, Chile; ${ }^{3}$ Universidad Nacional de Misiones, Argentina; ${ }^{4}$ National Cancer Institute, USA; ${ }^{5}$ Universidad Complutense de Madrid, Espana

Tympanoctomys barrerae, is a desert specialist restricted to salt flats of west central Argentina. Its 102 chromosome karyotype, DNA content and exceedingly large sperms have suggested a tetraploid origin. Male meiosis of $T$. barrerae was studied by electron-microscopy of surface- spread synaptonemal complexes (SC) and standard cytogenetics, in seven males. Synapsis with regular bivalent formation and no evidence of multivalents, was found. Nevertheless, gross inequalities in length of the lateral elements of SCs were observed, producing ca. 19-22 heteromorphic bivalents, also present at diakinesis. In G-banded mitotic chromosomes, the number of heteromorphic pairs was consistent with the meiotic results. Regular male meiotic behaviour (bivalent formation) of $T$. barrerae does not support a polyploid status of the species at the chromosomal level, although the high degree of chromosomal heteromorphism is unusual for mammals and suggestive of hybridsation (and perhaps, allopolyploidisation). Indeed, allotetraploids may undergo evolutionary diploidisation but, how is the high heteromorphic status mantained in the case of $T$. barrerae? One answer is that the observed chromosomal polymorphisms are not different from those observed in many animal species, and are neutral or mantained by balancing selection. The other, proposes $T$. barrerae as a polyploid of hybrid origin: heteromorphic autosomal pairs would be homeologous chromosomes from the parent species. But, how is this multiple heteromorphism mantained through meiotic segregation? A further hypothesis predicts multiple reproductive interactions of the putative parent species, along evolutionary time. Polyplody notwithstanding, $T$. barrerae presents several challenges to animal evolutionary cytogenetics.

\section{L64}

\section{Reconstruction of karyotype evolution in Arabidopsis thaliana and its close relatives by multicolour chromosome painting}

\author{
M. Lysak ${ }^{1}$, R. Schmidt ${ }^{2}$ A. Pecinka ${ }^{1}$, \\ J. Fuchs ${ }^{1}$ and I. Schubert ${ }^{1}$ \\ ${ }^{1}$ Institute of Plant Genetics and Crop Plant \\ Research (IPK), Gatersleben, Germany; ${ }^{2} \mathrm{Max}$ \\ Planck Institute of Molecular Plant Physiology, \\ Golm, Germany
}

The model plant Arabidopsis thaliana is one of the few species of the Brassicaceae family with 
chromosome number $2 \mathrm{n}=10$. Little is known about evolutionary history of the Arabidopsis karyotype and processes that contributed to the variation of basic chromosome numbers $(n=5-8)$ in the genus Arabidopsis and related taxa. The unique organization of the $A$. thaliana genome and the availability of chromosome-specific BAC libraries allowed multicolour painting of all Arabidopsis chromosomes and comparative painting studies in related species with a similar genome structure.

For the first time, comparative chromosome painting was successfully used to identify all chromosomes of an entire complement in genomes of related plant taxa. Multicolour FISH using Arabidopsis BAC contigs arranged according to the comparative genetic map between $A$. thaliana and Capsella rubella $(\mathrm{n}=8)$ revealed homeologous chromosomes/chromosome regions in pachytene complements of five cruciferous species with 6 to 8 chromosome pairs (Hornungia alpina $n=6$, Turritis glabra $\mathrm{n}=6$, Neslia paniculata $\mathrm{n}=7$, Pseudoarabidopsis toxophylla $\mathrm{n}=7$ and $A$. lyrata $\mathrm{n}=8$ ). The chromosome homeology pattern found for both, $C$. rubella and $A$. lyrata, was identified as the ancestral karyotype which became rearranged in other taxa in connection with chromosome number reduction from $n=8$ to 5 . Recurrent chromosome number reduction has been accompanied with gross genome reshuffling and genome size decrease. We have reconstructed diverse evolutionary scenarios including chromosome translocations, fusions and inversions that led to diversification of chromosome numbers from $\mathrm{n}=8$ to $\mathrm{n}=7,6$, and $\mathrm{n}=5$.

\section{L65}

\section{Karyological relationships between birds and reptiles inferred from comparative gene mapping}

Y. Matsuda ${ }^{1}$, C. Nishida-Umehara ${ }^{1}$, H. Tarui ${ }^{2}$, A. Kuroiwa ${ }^{1}$, K. Yamada ${ }^{1}$, T. Isobe ${ }^{1}$, J. Ando ${ }^{1}$, T. Saito ${ }^{3}$ and K. Agata $^{2}$

${ }^{1}$ Center for Advanced Science and Technology, Hokkaido University, Sapporo, Japan; ${ }^{2}$ RIKEN Center for Developmental Biology, Kobe, Japan; ${ }^{3}$ National Institute of Radiological Sciences, Chiba, Japan
The karyotypes of birds, turtles and snakes are characterized by two distinct chromosomal components, macrochromosomes and microchromosomes. This close karyological relationship between birds and reptiles has long been the topic of speculation among cytogenetists and evolutionary biologists, however there is no evidence for orthology at the molecular level. To compare chromosome organization between birds and reptiles, we constructed comparative cytogenetic maps of the Chinese soft-shelled turtle (Pelodiscus sinensis) and the Japanese four-striped rat snake (Elaphe quadrivirgata) with EST clones, which were isolated from cDNA libraries of the two species. Chromosome homology between the turtle and chicken chromosomes was highly conserved, the six largest chromosomes being almost equivalent each other. In contrast, the conserved linkage homology to chicken chromosomes was lower in the snake than that in the turtle. The turtle chromosome 6 and snake chromosome 2 revealed the linkage homology with the chicken sex $\mathrm{Z}$ chromosome. These results suggest that the avian and turtle genomes have been well conserved for over 250 million years in the phylogeny of Arcosauria, and that the avian and snake sex $\mathrm{Z}$ chromosomes were derived from the different chromosomes of the common ancestor. The present approach for comparative mapping using an extensive number of ESTs is a new strategy to investigate chromosome homologies between reptiles and birds. The disposition of conserved chromosome segments among reptiles, birds and mammals provides a clue for clarifying the phylogenetic hierarchy of vertebrate genome evolution.

\section{PO:06:37 \\ On the distribution of the interstitial telomeric sites and chromosome evolution in birds}

\section{S. Derjusheva ${ }^{1}$, A. Kurganova ${ }^{1}$, F. Habermann ${ }^{2}$ and E. Gaginskaya ${ }^{1}$ \\ ${ }^{1}$ Biological Institute of St. Petersburg University, St. Petersburg, Russia; ${ }^{2}$ Technical University of Munich, Germany}

Recently, chicken macrochromosome painting probes were successfully applied to chromosomes 
of sixteen avian species. The species belonged mainly to the order Galliformes, a few other avian orders were represented by a single species per order. In our study, we used chicken macroand microchromosomal paints to analyze homologies between chromosomes of the chicken ( $\mathrm{Gal}$ lus gallus domesticus: Galliformes), pigeon (Columba livia: Columbiformes), chaffinch (Fringilla coelebs: Passeriformes) and redwing (Turdus iliacus: Passeriformes). It was found that in investigated birds, individual chromosomes, with a few exceptions, remain chromosomal integrity of ancestral synteny. We revealed that chicken chromosome 4 paint hybridized with chromosome 4 and one pair of microchromosomes in pigeon, redwing and chaffinch. These data present an evidence of assumption that chicken $4 \mathrm{q}$ represents an ancestral type of avian chromosome 4. We showed also that fission of chicken chromosome 1 seems to be typical of passerine karyotypes. Using FISH-mapping, we analyzed the distribution of TTAGGG-repeat in pigeon, chaffinch and redwing karyotypes. Interstitial telomeric sites (ITSs) were found to localize in centromeric and/or interstitial CMA-positive bands on certain chromosomes of all investigated species. We did not find any correlation between the distribution of ITSs and chromosome rearrangements revealed by comparative chromosome painting. In chaffinch, ITSs co-localize with a tandem repeat GS (AF427996, AF427997), monomers of which contain internal TTAGGG motif. Our data clearly demonstrate that, at least in the chaffinch karyotype, interstitial telomeric sequences are generated by the expansion of TTAGGG-microsatellite into monomers of satellite DNA. This work was supported by RFBR (project 02-04-49980).

\section{PO:06:38}

\section{Towards an understanding of genome size evolution in Cypripedium (Orchidaceae)}

M. Fay ${ }^{1}$, P. Cook ${ }^{1}$, J. Greensmith ${ }^{1}$, R. Cowan ${ }^{1}$, L. Hanson ${ }^{1}$ and I. Leitch ${ }^{1}$

${ }^{1}$ Jodrell Laboratory, Royal Botanic Gardens, Kew, Richmond, Surrey, TW9 3AB, UK
The genus Cypripedium (Cypripedioideae; Orchidaceae) consists of approximately 45 species, and we have carried out phylogenetic analyses using matK and the trnL-F region of plastid DNA and the ITS region of nuclear ribosomal DNA. Most species of Cypripedium (including the native lady's slipper orchid, C. calceolus) have large genomes (in C. calceolus, $1 \mathrm{C}=32.4 \mathrm{pg}$ ). As a result of these large genomes, AFLP fingerprints are suboptimal, and are characterised by a few strongly amplifying bands and many unscorable, weakly amplifying bands. The strong bands are present in all individuals within species, and in some cases are shared between species. We have investigated the use of cloned copies of these bands as a means of identifying high-copy segments of DNA, some of which have now been shown to be fragments of retrotransposons. Combining these data with the phylogenetic hypotheses from sequence data and FISH, we will be able to gain a greater understanding of genome size evolution in Cypripedium.

\section{PO:06:39}

\section{Origin of $B$ and sex chromosomes in plants}

\section{H. Goswami ${ }^{1}$}

${ }^{1}$ Department of Genetics, Barkatullah University, Bhopal, India

Despite discovery of a sex chromosome in a plant in 1917 by Allen (Sphaerocarpus donelli) not many plants have been known to possess sex chromosomes. Among genera, sex chromosomes are known in Fucus ; Sphaerocarpus and Marchantia ; Isoetes pantii; Cycas and Ginkgo and several genera among Cucurbits and Melandrium. This paper presents a 'modus operandi' for the origin of a sex chromosome in natural populations of aquatic pteridophytic weed Isoetes pantii Goswami (1975) suggesting molecular mechanisms operative within the genome wherein a 'sexchromosome has evolved denovo'. Following events appear to be directly correlated with the 'appearance' of sex chromosome(s) recurrent heterochromatinisation, chromosome breaks, translocations and chromosome polymorphisms. Sex chromosomes are very rare but $\mathrm{B}$ chromosomes 
are common in almost all groups of plants (Jones $\&$ Rees, 1982). Two primary source for Bchromosomes have been considered. (A) Either an intragenomic fragement acquires the characteristics of a B or (B) Interspecific hybridization provides foreign DNA that evolves into a B chromosome. Isoetes pantii (natural hybrid) infact supports combination of both, hybridization theory as well as acquisition of Bchromsomes as foreign DNA. As per this hypothesis the B-chromosomes from both the parents viz: I. coromandelina and I. sampathkumarini have contributed to the genomic reshuffle thereby triggering appearance (origin) of X chromosome(s). Comparative studies on DNA methylation also indicate inherent role of genomic imprinting.

Goswami H.K. 1975. Cytologia 40: 543-552.

Goswami H.K. 2001 Annals de Genetique 44: 392

Jones R.N. \& Rees H.C. 1982 B-chromosomes Acad Press, London.

\section{PO:06:40}

\section{Reconstruction of karyotype changes during the evolution of neotropical rodents (Akodontini, Muridae, Rodentia) by cross-species chromosome painting}

\section{Hass' ${ }^{1}$, I. Sbalquiero ${ }^{2}$ and S. Müller ${ }^{1}$}

${ }^{1}$ Department Biology II, Human Genetics, Ludwig-Maximilians University, Munich, Germany; ${ }^{2}$ Departamento de Genética, Universidade Federal do Paraná, Curitiba, Brasil

Rodent genome and chromosome organization is a major focus of basic and applied research since certain members of this mammalian order are among the most important biomedical model species. Despite this fact, very little is known about the evolution of rodent chromosomes. Evidence from comparative sequence analysis, however, revealed that mouse and rat have acquired numerous chromosome rearrangements compared to other mammalian orders, whereas Zoo-FISH experiments indicated that the squirrel conserved most of the ancestral mammalian chromosomal syntenies. In order to obtain a deeper insight into the evolution of the karyotypes of neotropical members of the family Muridae we investigated Akodon cursor, $2 \mathrm{n}=14$, 15 and 16 and $A$. montensis, $2 \mathrm{n}=24$ and 25 (genus Akodon) by cross-species chromosome painting employing mouse and Chinese hamster chromosome-specific painting probes.

\section{PO:06:41}

\section{Human chromosomal Q-heterochromatin regions in individuals of various age}

\author{
A. Ibraimov ${ }^{1}$ \\ ${ }^{1}$ National Center of Cardiology and Internal \\ Medicine, Togolok Moldo str., 3, Bishkek, \\ 720040, Kyrgyzstan, C.I.S.
}

The quantitative content of chromosomal Q-heterochromatin regions (Q-HRs) was studied in individuals of different age groups taking into account their racial and ethnic affiliation. It was shown that chromosomal Q-HRs are most numerous in the genome of neonates, while they are the least numerous in the genome of elderly subjects (aged 60 years and older) regardless of the ethnic features of the individuals studied. Our data indicate that 1) in a population there is a clear-cut tendency towards a decrease in the number of chromosomal Q-HRs with age, regardless of racial and ethnic features on the individuals; 2) of all the age groups the genome of neonates contains the greatest number of Q-HRs; 3 ) decreases in the number of Q-HRs with age are not due to the 'loss' of Q-heterochromatin on individual loci or chromosomes, but occur simultaneously in all the seven Q-polymorphic autosomes, in keeping with all our previous observations. It should be noted that decreases in the number of chromosomal Q-HRs with age do not occur smoothly apparently. Thus, according to our data, in the Kyrghyz and Russian populations $39.6 \%$ and $47.6 \%$ of chromosomal Q-HRs 'disappeared' by the age of 60 years, respectively. Of them the portion of new-born Kyrghyz and Russian infants amounted to $70.6 \%$ and $58.4 \%$, respectively. It is supposed that the lesser amount 
of Q-HRs in the genome of elderly subjects is due to the selective advantage in their survival to old age. The possible selective value of chromosomal Q-HRs is discussed.

\section{PO:06:42}

\section{Chromosomal Q-heterochromatin variability in neonates deceased during first year of age}

\author{
A. Ibraimov ${ }^{1}$ \\ ${ }^{1}$ National Center of Cardiology and Internal \\ Medicine, Togolok Moldo str., 3, Bishkek, \\ 720040, Kyrgyzstan, C.I.S.
}

There is analyzed the amount of chromosomal Q-heterochromatin regions (Q-HRs) in a genome of neonates deceased during first years of life. Chromosome preparations were made from umbilical-cord blood with the subsequent Qstaining in 145 Kyrghyz and 37 Russian neonates. During the first year of life $17 \mathrm{Kyrghyz}$ and 5 Russian neonates have deceased from various diseases in stationary conditions, the diagnosis of which is confirmed by pathological anatomical dissection. Mean numbers of Q-HRs per individual in newborn population were $3.16 \pm 0.13$ in Kyrghyz and $3.59 \pm 0.23$ in Russian, whereas in neonates died $=4.58 \pm 0.23$ and $=4.80 \pm 0.37$ respectively. Neonates are characterized by a high range of variability in the distribution of Q-HRs (from 0 up to 7). But died neonates, besides high value, differ by extremely narrow range of variability of Q-HRs in population: number of Q-HRs in a karyotype changes from 4 up to 6. Q-HRs in all samples studied are encountered with an expected frequency on all the potentially Q-polymorphic autosomes, i.e. in no group there was preferential Q-HR localization, and this is again fsuggesting that Q-heterochromatin is not locusspecific material in the genome. It is supposed that individuals with the greatest amount of Q-HRs in the newborn population have greater probability to die in the first years of life other conditions being equal.

\section{PO:06:43}

\section{Condensed chromatin and cell thermoregulation}

\author{
A. Ibraimov ${ }^{1}$ \\ ${ }^{1}$ Laboratory of Human Genetics, National Center \\ of Cardiology and Internal Medicine, Togolok \\ Moldo str., 3, Bishkek, 720040, Kyrgyzstan, \\ C.I.S.
}

Based on own original investigations of chromosomal Q-heterochromatin regions variability in human populations, as well as on the analysis of existing literary data on the condensed chromatin (CC), structure of interphase nucleus and redundant DNA in the genome of higher eukaryotes, an attempt is made to justify a view about possible participation of $\mathrm{CC}$ in cell thermoregulation. CC (tissue-specific condensed chromatin, facultative and constitutive heterochromatin regions), being the densest domains in a cell, apparently conducts heat between the cytoplasm and nucleus when there is a difference in temperature between them. The assumed heat conductivity effect of $\mathrm{CC}$ is stipulated by its principal features: condensed state during the interphase, association with the lamina and the inner nuclear membrane, replication at the end of the $S$ period of a cell cycle, formation of the chromocenter, genetic inertness, wide variability in the quantitative contents both within and between species. Our idea proposed in this paper is reduced to the evolution of genome structure and physiology of the whole organism in higher eukaryotes going in parallel to counteract changes of temperature in the ambient environment for more effective preservation of constancy of temperature of the internal environment. The outcomes of such parallel evolution were: a) appearance of different kinds of $\mathrm{CC}$ (C- and Q-heterochromatin, $\mathrm{G}+$ and $\mathrm{Q}+$ bands, sex chromatin body), at a genome level the effect of which is generally subject to laws of physics; b) formation at an organism level of a complex organ-based physiological system of thermoregulation. 
PO:06:44

\section{Molecular cloning and characterization of novel highly repetitive DNA sequences of the greater long-tailed hamster (Tscherskia triton, Cricetridae)}

\author{
E. Kamimura ${ }^{1}$, K. $\operatorname{Yamada}^{2}$, K. Tsuchiya ${ }^{3}$, \\ C. Nishida-Umehara ${ }^{2}$ and Y. Matsuda ${ }^{2}$ \\ ${ }^{1}$ Laboratory Animal Center, Yamagata \\ University School of Medicine, Yamagata, Japan; \\ ${ }^{2}$ Laboratory of Animal Cytogenetics, Center for \\ Advanced Science and Technology, Hokkaido \\ University, Sapporo, Japan; ${ }^{3}$ Laboratory of Wild \\ Animals, Department of Zootechnical Sciences, \\ Faculty of Agriculture, Tokyo University of \\ Agriculture, Atsugi, Japan
}

The diploid chromosome number of the greater long-tailed hamster (Tscherskia triton, Cricetridae) is 28 , and its karyotype consists of eleven pairs of acrocentric chromosomes, two pairs of small metacentric chromosomes, and middlesized subtelocentric $\mathrm{X}$ and metacentric $\mathrm{Y}$ chromosomes. We isolated a new family of sitespecific highly repetitive DNA sequences from TaqI-digested genomic DNA of the greater longtailed hamster, and characterized them by chromosome in situ hybridization, sequence analysis and filter hybridization. The TaqI-family of repetitive sequences was classified into two types by their genome organization and chromosomal distribution; 1) centromere-specific repetitive sequence, and 2) sex chromosome-specific repetitive sequence. The size of the centromere-specific repetitive sequence was $112 \mathrm{bp}$, and the element was arranged into large tandem arrays and localized to centromeric C-positive regions of all acrocentric autosomes and subtelocentric $\mathrm{X}$ chromosome. The sex chromosome-specific repetitive sequence, which was composed of 30-32bp internal repeats, was distributed on the short arm of $\mathrm{X}$ chromosome and the long arm of $\mathrm{Y}$ chromosome. Both the centromeric satellite DNA and the sex chromosome-specific repetitive sequence did not cross-hybridize with genomic DNAs of other seven Cricetinae species, Mesocricetus auratus (diploid number: $2 \mathrm{n}=44$ ), $M$. brandti $(2 \mathrm{n}=44)$, Cricetulus griseus $(2 \mathrm{n}=22)$, C. migra- torius $(2 \mathrm{n}=22)$, Phodopus sungorus $(2 \mathrm{n}=28), P$. campbelli $(2 \mathrm{n}=28)$, and $P$. roborovskii $(2 \mathrm{n}=34)$, and one Calomyscinae species, Calomyscus mys$\operatorname{tax}(2 \mathrm{n}=44)$. These results suggest that both the repetitive DNA sequences were species-specific and rapidly diverged. We compare them with the site-specific repetitive sequences cloned from M. auratus, and discuss the molecular evolution of repetitive sequences in the Cricetinae.

\section{PO:06:45}

\section{Genome downsizing in polyploid plants}

\section{Leitch ${ }^{1}$ and M. Bennett ${ }^{1}$}

\author{
${ }^{1}$ Jodrell Laboratory, Royal Botanic Gardens,
} Kew, Richmond, Surrey TW9 3DS, UK

Polyploidy is a key factor in angiosperm evolution leading to the formation of new species. All else being equal, polyploids are expected to have larger C-values (DNA amount in the unreplicated gametic nucleus) than their diploid progenitors, increasing in direct proportion with ploidy. This expectation is observed in some polyploid series, especially those newly formed, but there are examples suggesting that $\mathrm{C}$-values in particular polyploids are lower than expected. The availability of the angiosperm DNA C-values database (http://www.rbgkew.org.uk/ cval/homepage.html) has allowed this question to be addressed across a broad sample of angiosperms and has revealed striking results deviating from expectation: (i) Mean 1C DNA amount did not increase in direct proportion with ploidy, and (ii) Mean DNA amount per 'basic genome' (= 2C divided by ploidy) tended to decrease with increasing ploidy. The same striking results were also observed for six monophyletic subgroups (monocots, all eudicots, Caryophyllales, core eudicots, asterids and rosids) and six families (Poaceae, Alliaceae, Ranunculaceae, Fabaceae, Solanaceae, Asteraceae). These results suggest that DNA loss following polyploid formation, or genome downsizing, maybe a widespread phenomenon of considerable biological significance. Recent advances in understanding the molecular events which take place following polyploid formation, together with new data on how DNA amounts can change, provide some insights into 
how genome downsizing may take place. In searching for the nature of the evolutionary forces which may drive DNA loss, the basic genome size itself appears to play some role in determining the extent of genome downsizing following polyploidization

\section{PO:06:46}

\section{Genome size evolution across land plants (Embryophyta)}

\section{Leitch ${ }^{1}$, D. Soltis ${ }^{2}$, P. Soltis ${ }^{3}$ and M. Bennett ${ }^{1}$}

${ }^{1}$ Jodrell Laboratory, Royal Botanic Gardens, Kew, Richmond, Surrey TW9 3DS, UK; ${ }^{2}$ Department of Botany and the Genetics Institute, University of Florida, Gainesville, Florida 32611-5826 USA; ${ }^{3}$ Florida Museum of Natural History and the Genetics Institute, University of Florida, Gainesville, Florida 32611-7800 USA

Genome sizes (DNA C-values) of land plants (Embryophyta; comprising bryophytes, lycophytes, monilophytes, gymnosperms and angiosperms) range over 1000 -fold from c. $0.1 \mathrm{pg}$ to $127.4 \mathrm{pg}$. To understand the evolutionary significance and to investigate the evolutionary forces responsible it is essential to evaluate the phylogenetic component of this huge variation. Such an analysis is timely given the recent improved consensus of relationships between and within different land plant groups, and increased availability of $\mathrm{C}$-values (data are currently available for over 4500 species, the majority of which are accessible in the Plant DNA C-values database (release 2.0, Jan. 2003; http://www.rbgkew.org.uk/cval/homepage.html). Consequently, $\mathrm{C}$-values were superimposed onto well-supported phylogenies and analysed by character state reconstruction using MacClade. Using the definitions of very small $(1.4 \mathrm{pg})$, small $(3.5 \mathrm{pg})$, intermediate (3.5 to $14.0 \mathrm{pg}$ ); large (14 pg) and very large $(35 \mathrm{pg})$ to characterise C-values (see Soltis et al. 2003 Am. J. Bot 90: 1596-1603), analysis revealed the ancestral $\mathrm{C}$-value of all land plants was probably very small, a state that also characterised ancestral angiosperms and bryophytes. In contrast gymnosperms and the majority of monilophytes were characterised by intermediatesized ancestral genomes. More in-depth analyses within each land plant group provided evidence for several independent increases and decreases in genome size. Thus in agreement with several focused studies within angiosperm families and genera showing that $\mathrm{C}$-values may both increase and decrease, it is apparent that this dynamic pattern of genome size evolution is repeated on a broad scale across land plants.

\section{PO:06:47}

\section{Dynamic mobility of the rDNA spacer around the genome in the evolution of Nicotiana}

\author{
K. Lim', K. Skalicka ${ }^{2}$, B. Koukalova ${ }^{2}$, \\ R. Volkov ${ }^{3}$, V. Hemleben ${ }^{3}$, A. Leitch ${ }^{1}$ and \\ A. Kovarik ${ }^{2}$
}

${ }^{1}$ Queen Mary University of London, UK;

${ }^{2}$ Institute of Biophysics, Brno, Czech Republic;

${ }^{3}$ Center of Plant Molecular Biology, Tübingen, Germany

The 26-18S rDNA intergenic spacer (IGS) of Nicotiana contains A1/A2 subrepeats. To our surprise we observed large amounts of subrepeats (termed A1/A2 satellite) outside of rDNA loci in species of Nicotiana section Tomentosae but not from species in other sections. The chromosomal location, patterns of genomic dispersion and copy numbers of A1/A2 satellite varied considerably between species in section Tomentosae. At the species level we found differences in the chromosomal distribution of the $\mathrm{A} 1 / \mathrm{A} 2$ satellite even between accessions of $N$. tomentosiformis suggesting a rapid rate and large-scale sequence redistribution within timeframes shorter than speciation events. The A1/A2 satellite and the A1/A2 sequences at the rDNA locus can be separated by gel electrophoresis and units from both genomic fractions sequenced. We were astonished to find that the sequences occurred in the same populations. The best model to fit the data is that there is recurrent integration of repeats into the genome from the rDNA loci and that this is associated with copy number expansions, contractions and deletions, all occurring 
over relatively short time frames (probably less than 60,000 years). There was also a trend towards the elimination of the A1/A2 satellite in $N$. tabacum (tobacco, 10,000 years old), an allotetraploid with parents closely related to the diploids $N$. sylvestris and $N$. tomentosiformis. This process may have already commenced in an S3 generation of synthetic tobacco.

\section{PO:06:48}

\section{Chromosome triplications indicate that the tribe Brassiceae is descended from a hexaploid ancestor: evidence from comparative chromosome painting}

M. Lysak ${ }^{1}$, M. Koch ${ }^{2}$, A. Pecinka ${ }^{1}$ and I. Schubert ${ }^{1}$

${ }^{1}$ Institute of Plant Genetics and Crop Plant Research (IPK), Gatersleben, Germany; ${ }^{2}$ Institute for Plant Sciences, University of Heidelberg, Heidelberg, Germany

Based on comparative genetic mapping between Arabidopsis thaliana and Brassica species, it was suggested that diploid Brassica genomes are descended from a hexaploid ancestor and this might be valid also for other members of the Brassiceae tribe. To prove this assumption we employed comparative chromosome painting using a BAC contig spanning $8.7 \mathrm{Mb}$ of the bottom arm of Arabidopsis chromosome 4 in 21 species traditionally placed in the tribe Brassiceae. With the exception of the genera Calepina and Conringia, the analyzed BAC contig labels segments of three (to four) chromosome pairs in species with $2 \mathrm{n}=14,16,18,20,22,28,30$ and six chromosome pairs in amphidiploid Brassica species with $2 \mathrm{n}=34,36$ and 38. The multiple copies correspond structurally to the BAC contig of $A$. thaliana or more often they are rearranged in a species-specific manner. Our data bring new evidence that most of the diploid Brassiceae species (including diploid brassicas) are descended from an Arabidopsis-like ancestor which underwent polyploidization (hexaploidy) followed by subsequent chromosome fusions/fissions and inversion/translocation events. We generated sequence data for ITS 1 and 2 regions of nuclear rDNA and the plastidic 5-trnL(UAA)-trnF(GAA) region to infer a robust phylogenetic hypothesis based on two independent markers. The preliminary results confirm the monophyletic origin of the Brassiceae, however, it is also suggested that Conringia and Calepina might be placed outside this clade. This is congruent with the absence of chromosome triplication.

\section{PO:06:49}

\section{Karyotypic evolution of hamster species (Cricetinae, Muridae, Rodentia) inferred from comparative FISH analyses}

\author{
K. Matsubara ${ }^{1}$, K. Tsuchiya ${ }^{2}$ and Y. Matsuda ${ }^{1}$ \\ ${ }^{1}$ Lab. Anim. Cytogenet., Center for Advanced Sci. \\ and Tech., Hokkaido Univ.; ${ }^{2}$ Lab. Wild Anim., \\ Fac. Agri., Tokyo Univ. Agri., Japan
}

Hamster species belonging to the Cricetinae are distributed from South Europe to East Asia and 18 species of these taxa are now recognized in this subfamily. The diploid chromosome number of Cricetinae species is $2 \mathrm{n}=22-44$, and their fundamental number ranges from 44 to 80 . The diversity of karyotypes has been achieved by the accumulation of chromosome rearrangements that occurred during evolution, and thus it has been possible to define the history of karyotypic changes by comparing the karyotypes among the extant species. In this study, to identify the chromosomal homologous regions among the six Cricetinae species, Chinese hamster (Cricetulus griseus), Armenian hamster (C. migratorius), Syrian hamster (Mesocricetus auratus), Djungarian hamster (Phodopus campbelli), Desert hamster (P.roborovskii) and Greater long-tailed hamster (Tscherskia triton), we conducted comparative chromosome painting with the chromosomespecific probes of Chinese hamster. Comparative analysis revealed that many chromosome rearrangements had occurred among the six species. The chromosomal locations of the $5 \mathrm{~S}$ and $18 \mathrm{~S}$ $28 \mathrm{~S}$ ribosomal RNA (rRNA) genes were also compared. The chromosomal location of the $5 \mathrm{~S}$ rRNA genes on the region homologous to Chinese hamster chromosome 3 was highly 
conserved in all the species. In contrast, there was variation in the location of the $18 \mathrm{~S}-28 \mathrm{~S}$ rRNA genes. The karyological data were compared with the molecular phylogenetic data, and karyotypic evolution in Cricetinae is discussed.

PO:06:50

\section{Evolution of the heterochromatin organization in the Drosophila melanogaster species group}

L. Monti-Dedieu' ${ }^{1}$, S. Aulard ${ }^{2}$, N. Chaminade ${ }^{2}$, C. Montchamp-Moreau ${ }^{2}$ and F. Lemeunier ${ }^{2}$

${ }^{1}$ IUFM, Paris, France; ${ }^{2} \mathrm{CNRS}$, Populations, Génétique, Evolution, Gif, France

In Drosophila, the heterochromatic sequences are located to restricted regions of the chromosomes and subjected to a very rapid evolution. Previous data on karyotype descriptions were mainly obtained by classical cytogenetic techniques. Using molecular approach, we are now able to describe more precisely the chromosomal morphology and the distribution of heterochromatin. Because the ribosomal DNA genes (rDNA) are embedded in the heterochromatic regions, they can be used as convenient markers of the evolution of genes in heterochromatin.

In order to understand the occurring of heterochromatic rearrangments within the melanogaster group, we used fluorescent in situ hybridisation (FISH) and DAPI staining. We studied species belonging to 8 of the 11 subgroups from the melanogaster group.

The results show that the heterochromatin distribution is species specific and that the $\mathrm{Y}$ chromosome is mainly concerned by rearrangements. They suggest also that the ancestral configuration consists of one nucleolus organizer (NOR) on each sex chromosome. This scheme is found in most subgroups but some of them present exceptions that will be discussed in the light of species evolution.

PO:06:51

Position studies in interphase nuclei of conserved chromosomes from different primate species

\section{Mora ${ }^{1}$, M. Garcia ${ }^{1}$ and M. Ponsa ${ }^{1}$}

${ }^{1}$ Institut de Biotecnologia i Biomedicina. Departament de Biologia Celular, Fisiologia i Immunologia. Universitat Autonoma de Barcelona, Spain

In this work, chromosomes conserved from the ancestor of Primates are studied to know its position in interphase nucleus in order to elucidate if the position is conserved in different species. For this purpose we have analysed human (HSA) chromosome 17 and 9 and its homologuos chromosomes in Lagothrix lagothricha (LLA) and Saimiri sciureus (SSC) cell lines. Although ancestral HSA 17 is conserved among the three species, ancestral HSA 9 is conserved in LLA and HSA but fusionated with another chromosome fragment in SSC.

Fibroblast cell lines from the three species were grown on slides, fixed and permeabilized maintaining the 3D structure. Nuclei were hybridised using human whole-chromosome probes and observed by laser confocal microscopy. Distances from the centre of each signal to the centre of the nucleus were measured and divided by its relative radius. Around 100 nuclei were analysed for each probe and species.

Results show that the average position of ancestral HSA 17 in human, LLA and SSC cell lines is $62.72 \%, 55.84 \%$ and $45.35 \%$ of the nuclear radius respectively, being the position of SSC significantly different. Average positions of ancestral HSA 9 in HAS, LLA and SSC are $49.08 \%$, $63.51 \%$ and $68.58 \%$ respectively, being HSA position significantly different.

Our data show that although both chromosomes are phylogeneticaly conserved its position in the nucleus is not always conserved. Results are discussed in relation to the size, morphology and phylogenetic parameters of each chromosome.

\section{PO:06:52}

\section{Comparative cytogenetic studies of regeneration organisms, newt and two planarian species}

T. Murakami ${ }^{1}$, Y. Umesono ${ }^{1}$, A. Sanchez ${ }^{2}$, C. Umehara ${ }^{3}$, Y. Matsuda ${ }^{3}$ and K. Agata ${ }^{1}$ 
${ }^{1}$ Center for Developmental Biology, Riken;

${ }^{2}$ Department of Neurobiology and Anatomy, University of Utah School of Medicine;

${ }^{3}$ Chromosome Research Unit, Hokkaido University

Freshwater planarian flatworms have high ability of regenerating complete organisms from tiny fragments of the bodies. This regenerative competence is based on stem cell population that is present in the adult planarian. Recently, to promote the molecular analysis of regeneration and stem cell regulation in planarians, over 9,000 ESTs has been generated from a clonal line of Dugesia japonica that is originated from Japan and also 3,000 unique ones from Schmidtea mediterranea that is originated from Spain and has been kept at U.S.A., respectively. Although distinctive results using this ESTs database have been developed independently, there is no data whether one clone generated from one species is able to fit others or not. Therefore, we need to confirm that these distinct family-level different flatworms can share the functional genes each other. Furthermore, chromosome research in planarian has been very few in this decade and there is no report FISH analysis for the organisms. Consequently, we established basic chromosome staining techniques followed by applied FISH analysis for both species. Then, we could make ideograms and it will be standard for identifying each gene localization. Telomere sequences were conserved and could observe larger signals on the end of all chromosome than other organisms. In $\mathrm{S}$. mediterranea there was translocation from the first chromosome to third ones in asexual strain. This is outstanding for revealing the mechanism of evolution of sexual reproduction. Finally we were able to hybridize 10 functional genes on each chromosome, and will establish the complete genome maps.

\section{PO:06:53}

\section{Comparative cytogenetic studies on karyotypic evolution in birds}

C. Nishida-Umehara ${ }^{1}$, M. Shibusawa ${ }^{1}$, Y. Shimada ${ }^{1}$,J. Ando ${ }^{1}$, J. Ishijima ${ }^{1}$, A. Fujiwara ${ }^{2}$, T. Murakami ${ }^{3}$, J. Masabanda ${ }^{4}$, D. Griffin ${ }^{4}$ and Y. Matsuda ${ }^{1}$
${ }^{1}$ Center for Advanced Science and Technology, Hokkaido University, Sapporo, Japan; ${ }^{2}$ Immunology Section, National Research Institute of Aquaculture, Fisheries Research Agency, Tamaki, Japan;

${ }^{3}$ RIKEN Center for Developmental Biology, Kobe, Japan; ${ }^{4}$ Department of Biological Sciences, Brunel University, Uxbridge, UK

Bird karyotypes are mainly classified into two types, the conservative karyotype and the variant karyotype. The conservative karyotype that is composed of two major components, macrochromosomes and microchromosomes, is common in most of avian orders and reptiles. The variant karyotypes are observed in the Accipitridae and Falconidae of Falconiformes, which have few microchromosomes and a large number of middle-sized chromosomes. To investigate the chromosome conservation and process of chromosome rearrangements in birds with the two different karyotypes on a molecular level, we compared chromosome homologies in birds of five orders (Struthioniformes, Tinamiformes, Galliformes, Strigiformes, Falconiformes) by comparative chromosome painting with chicken DNA probes of chromosomes 1-9 and Z. Each chicken probe painted one chromosome pair except for chromosome 4 paint that hybridized to one macrochromosome pair and a pair of microchromosomes in four ratite species of Struthioniformes (ostrich, common rhea, Darwin's rhea, double-wattled cassowary) and Tinamiformes (elegant crested tinamou). Also in Galliformes and Strigiformes, chromosome rearrangements have occurred in low frequency. In contrast to the chromosome conservation in the four orders, high frequencies of chromosome rearrangements were observed in the Accipitridae and Falconidae species. Chicken chromosome 1 probe painted six and two pairs of chromosomes in mountain hawk eagle of the Accipitridae and peregrine falcon of the Falconidae respectively, and chromosomes 2 and 3 probes painted three and four chromosome pairs in the eagle, and two and two pairs in the falcon, respectively. These results indicate that macrochromosomes have been divided by sequential chromosome rearrangements in these species. 
PO:06:54

\section{New results in Platyrrhini chromosome evolution based on comparative chromosome painting: The case of Aotus}

\author{
A. Ruiz-Herrera ${ }^{1}$, F. García ${ }^{2}$, M. Aguilera ${ }^{3}$, \\ M. Garcia ${ }^{1}$ and M. Ponsà ${ }^{1}$
}

${ }^{1}$ Departament de Biologia Cellular, Fisiologia i Immunologia, Universitat Autònoma de Barcelona. 08193, Cerdanyola del Vallès (Barcelona). Spain; ${ }^{2}$ Institut de Biotecnologia i de Biomedicina (IBB). Universitat Autonoma de Barcelona. 08193, Cerdanyola del Vallès (Barcelona). Spain; ${ }^{3}$ Grupo BIOEVO, Universidad Simón Bolívar (Caracas). Venezuela

The owl monkey, Aotus, is the unique genus from Subfamily Aotinae ( $F$. Cebidae, Platyrrhini). Taxonomy of the genus Aotus is especially intricate since cytogenetic studies based on G- and C-banding comparisons have characterized 18 different karyotypes, which chromosome diploid numbers range from 46 to 58 chromosomes. In an attempt to clarify the chromosomal relationships between Aotus species in relation to the rest of New World monkey species, we have used human chromosome specific-paints to delineate, for the first time, the homologous chromosomal segments present in Aotus.

Heparinized peripheral blood samples were taken from one male Aotus nancymai $(2 \mathrm{n}=54)$ and one male Aotus sp. $(2 \mathrm{n}=50)$ from Venezuela in order to obtain chromosome preparations. All two specimens were chromosomally characterized after sequential G- and C-banding. Whole chromosome paints for all human chromosome were used for FISH on Aotus metaphases.

With the exception of human chromosome Y, all human chromosome-specific painting probes are hybridized on all Aotus chromosomes. Based on G-banding comparisons and the fluorescent 'in situ' hybridizations results, the chromosomal homologies between two Aotus species have been established. From the putative ancestral Platyrrhini associations $(2 / 16,3 / 21,5 / 7,8 / 18,10 / 16$, $14 / 15)$, two of them, $2 / 16$ and $10 / 16$ were not found in the two Aotus species. In addition, some derived chromosomal associations were detected:
$1 / 3,1 / 16,2 / 12,2 / 20,4 / 15,5 / 15,7 / 11,9 / 15$, $10 / 11,10 / 22$; whereas $3 / 14$ and $9 / 17$ were found only in Aotus nancymai.

\section{PO:06:55}

\section{Effects of the genetic background, karyotype and sex of $\mathbf{R b}$ heterozygous mice (Mus musculus domesticus) on the nondisjunction rate in meiosis $I$}

M. Scascitelli ${ }^{1}$, F. Pacchierotti ${ }^{2}$, F. Spirito ${ }^{3}$, B. Gustavino ${ }^{1}$ and M. Rizzoni ${ }^{1}$

${ }^{1}$ Department of Biology, University of Rome, Tor Vergata, via della Ricerca Scientifica snc, 00133 Rome, Italy; ${ }^{2}$ Section of Toxicology and Biomedical Sciences, ENEA CR Casaccia, via Anguillarese 301, 00060 Rome, Italy; ${ }^{3}$ Department of Genetics and Molecular Biology, C. Darwin, University of Rome, la Sapienza, Piazzale Aldo Moro 5, 00185, Rome, Italy

The anaphase I nondisjunction rate of specific chromosome arms of Robertsonian metacentrics was estimated by dual-colour FISH (fluorescence in situ hybridisation). Spermatocyte II metaphases were obtained from laboratory mice (Mus musculus domesticus) heterozygous for two and three Robertsonian centric fusions.

Data from double heterozygotes were compared with previous ones from heterozygotes for the same number and type of $\mathrm{Rb}$ chromosomes, but obtained with a different mating scheme. It allowed to check the possible influence of the genetic background on the nondisjunction rate for specific chromosome arms and/or on the overall amount of aneuploid spermatocytes.

On the other hand the comparison of triple heterozygotes for $\mathrm{Rb}$ chromosomes involving different acrocentrics allowed to understand if alternative combinations of $\mathrm{Rb}$ centric fusions affect the NDJ rate of specific chromosomes and/or the incidence of aneuploidy during meiosis I.

We studied the overall frequency of aneuploid oocytes from individuals with multiple $\mathrm{Rb}$ heterozygous chromosomes: we found higher values compared to the male germ system with the same karyotype. Finally we analysed their role in an evolutionary context with mathematical models. 


\section{Telomeres Symposium}

L66

\section{Protection and maintenance of human telomeres}

\section{T. De-Lange}

The Rockefeller University, 1230 York Avenue, New York, NY 10021, USA

No abstract was submitted for this talk.

\section{L67}

\section{Dangerous entanglements: roles of telomeres in promoting S-phase progression}

\section{K.M. Miller, M. Ferreira and J. Cooper}

Telomere Biology Laboratory, Cancer Research UK, London WC2A 3PX, UK

Fission yeast Taz1 is the only known ortholog of the mammalian telomere proteins TRF1 and TRF2. Taz1 regulates telomeric DNA synthesis, position effects on nearby transcription, and meiotic chromosome movements. Furthermore, Tazl controls two types of detrimental associations between chromosome ends. First, Tazl acts with Rap1 to prevent DNA repair reactions from acting inappropriately on telomeres. Deletion of taz1+ or rap1+ leads to end-fusions during G1 arrest, when nonhomologous end-joining activities are elevated. Second, Tazl itself regulates replication fork progression through telomeres. Deletion of taz1+ leads to replication fork stalling at telomeres, while rap1 cells do not suffer this impediment. This replication defect is associated with telomeric entanglement at cold temperatures. These entanglements, which can be suppressed by a specific mutation in topoisomerase II, are distinct from covalent telomere fusions but nonetheless lead to chromosome breakage and missegregation. We will discuss the origin and resolution of this newly recognized threat to unprotected chromosome ends.
L68

\section{Regulation of telomerase and the mechanism of telomere length homeostasis}

\author{
J. Lingner ${ }^{1}$, M. Arneric ${ }^{1}$, K. Färstemann ${ }^{1}$, \\ C. Kelleher ${ }^{1}$, I. Kurth ${ }^{1}$, P. Sperisen ${ }^{1}$ and \\ T. Teixeira ${ }^{1}$
}

${ }^{1}$ Swiss Institute for Experimental Cancer Research (ISREC); CH-1066 Epalinges s/Lausanne, Switzerland

Telomerase activity is regulated at individual chromosome ends in cis, to achieve telomere length homeostasis. This regulation involves telomeric proteins and perhaps higher order DNA structures. Based on cellular studies, the human telomeric single-strand DNA binding protein protection of telomeres 1 (POT1) has been implicated in positive and negative regulation of telomerase. To determine its effects at the molecular level, we used recombinant POT1 and analyzed its effects on telomerase activity in vitro. We show that POT1 negatively effects telomerase activity by reducing the processivity of the enzyme, while it does not prevent telomerase from accessing the telomeric DNA primer. The negative regulation of telomerase by POT1 requires the DNA binding protein to be in molar excess over the primer suggesting that POT1 modulates telomerase processivity while bound to the DNA substrate. To analyze the mechanism of telomerase regulation in vivo we developed a method to measure telomere elongation at nucleotide resolution in Saccharomyces cerevisiae. We found that the number of nucleotides added to a telomere in a single cell cycle is independent of telomere length. Telomerase does not act on every telomere in each cell cycle, however. Instead it exhibits an increasing preference for telomeres as their lengths decline. Deletion of the telomeric proteins Rif1 or Rif2 gives rise to longer telomeres by increasing the frequency of elongation events. We conclude that telomere length homeostasis is achieved via a switch between telomerase extendible and non-extendible states. 
L69

\section{Telomere cytogenetics in senescence and ALT}

\author{
Y. Zou ${ }^{1}$, J. Shay ${ }^{1}$ and W. Wright ${ }^{1}$ \\ ${ }^{1}$ Univ. Texas Southwestern Medical Center, USA
}

Progressive telomere shortening is the counting mechanism that controls replicative aging. Using Q-FISH, we have identified the shortest telomeres in BJ normal human fibroblasts. We show that the shortest telomeres co-localize with DNA damage signals in senescent cells, and that the 10 shortest telomeres account for $>90 \%$ of all telomeric end-associations observed when senescent checkpoints are abrogated by expressing human papilloma virus $16 \mathrm{E} 6+\mathrm{E} 7$ proteins. We have also examined the timing of telomere replication using a newly developed CO-FISH technique. Yeast telomeres are late replicating and telomeres at each end of yeast chromosomes exhibit coordinated replication timing. In contrast, individual human and Indian Muntjac telomeres replicate at specific times throughout S-phase. Although replication timing of $\mathrm{p}$ or $\mathrm{q}$ arm telomeres of both chromosomal homologues is similar (e.g., maternal and paternal $2 \mathrm{p}$ telomeres replicate at approximately the same time), the timing of $p$ and $\mathrm{q}$ arms of individual telomeres are not coordinated (e.g., there is no coordination between $2 p$ and $2 \mathrm{q}$ replication). Finally, we will describe the presence of ultra-high frequency sister chromatid exchange within telomeres (T-SCE) in ALT cells. ALT cells use a recombination-based mechanism of telomere elongation in order to become immortal. In contrast to normal mortal or telomerase-expressing immortal cells, ALT cells show SCE at typically $>50 \%$ of all telomeric ends per metaphase.

\section{L70}

\section{Telomere maintenance in DNA} damage response deficient cells

\section{E. Cabuy ${ }^{1}$ and P. Slijepcevic ${ }^{1}$}

\footnotetext{
${ }^{1}$ Department of Biological Sciences, Brunel University, Uxbridge, Middlesex, UB8 3PH, UK
}

Telomeres are physical ends of chromosomes responsible for chromosome stability maintenance. The evidence is accumulating that telomere maintenance may be altered in cells with DNA damage response defects including cells from patients with ataxia telangiectasia (AT), Nijmegen breakage syndrome (NBS) and Fanconi anemia (FA). To investigate the effect of DNA damage response deficiencies on telomere maintenance further we used several primary fibroblast cell lines from radiosensitive patients with novel DNA damage response defects including defects in Artemis, ligase I, ligase IV, and two unknown factors that cause radiosensitivity. We monitored the rates of telomere shortening in the above cell lines using Southern blot and flowFISH techniques. Our results revealed accelerated telomere shortening in cells from the above patients in comparison with control cells. In addition we found evidence of telomere dysfunction in cells with accelerated telomere shortening. We also analyzed telomere maintenance in mouse embryonic stem cells deficient in BRCA1. Similarly we observed accelerated telomere shortening and end-to-end chromosome fusions in these cells. We are currently attempting to suppress BRCA1 in human mammary epithelial cells using siRNA and analyze effect of BRCA1 suppression on human telomeres.

\section{PO:06:56}

\section{Physical mapping of the terminal regions of individual rye chromosomes} O. Alkhimova ${ }^{1}$, J. Dolezel ${ }^{2}$ and A. Vershinin ${ }^{3}$

${ }^{1}$ Institute of Molecular Biology and Genetics, 03143 Kiev, Ukraine; ${ }^{2}$ Institute of Experimental Botany, 77200 Olomouc, Czech Republic; ${ }^{3}$ Institute of Cytology and Genetics, 630090 Novosibirsk, Russia

Telomeres are specialized chromosome structure, which play important role in chromosome stability and behaviour. Together with subtelomeres, they form terminal regions of chromosomes. These are dynamic regions and might help organisms to adapt to new environmental conditions and promote genomic rearrangements. Large heterochromatic blocks, which are present near the telomeres 
of rye chromosomes, are notoriously difficult for physical mapping because of enrichment by different types of repetitive DNA sequences. We used wheat-rye addition lines and performed fluorescence in situ hybridization (FISH) on metaphase flow-sorted chromosomes and extended DNA fibers for comparison of the terminal regions of the individual rye chromosomes. Each wheat-rye addition line produced a chromosome-specific largescale organization of the tandem repeat arrays. The FISH signals on DNA fibers showed the multiple patterns of co-linear monomers arrangement of repetitive families as well as of authentic telomeric probe from Arabidopsis. The primary structure of some spacer sequences revealed the scrambled regions of similarity to various known repetitive elements. Using PCR analysis, we determined the DNA sequences adjacent to the tandem repeats array (spacers). They are enriched of short direct, complementary, inverted and symmetrical repeats which appear to be associated with recombination events. These spacers may be a powerful source of tandem array rearrangements. We mapped some of them on metaphase stretched chromosomes, and the distribution of the signals was in good agreement with the localization of the breakpoints for deletions and for translocations of $1 \mathrm{R}$ long arm with different wheat chromosomes.

This work was supported by the INTAS grant (03-51-5908).

\section{PO:06:57}

\section{Distribution of intrachromosomal telomeric sequences (ITS) on Rattus norvegicus chromosomes}

\section{N. Camats' ${ }^{1}$ A. Ruiz-Herrera ${ }^{3}$, F. Garcia' ${ }^{1}$, J. Egozcue $^{3}$ and M. Garcia ${ }^{2}$}

${ }^{1}$ Institut de Biotecnologia i Biomedicina (IBB). Dept de Biologia Celular, Fisiologia i Immunologia. Universitat Autónoma de Barcelona (UAB). Spain; ${ }^{2}$ Unitat de Biologia, Facultat de Medicina, Departament de Biologia Cellular, Fisiologia i Immunologia, Universitat Autónoma de Barcelona (UAB), Spain.;

${ }^{3}$ Unitat de Biologia, Facultat de Ciències, Departament de Biologia Cellular, Fisiologia i Immunologia, Universitat Autónoma de Barcelona (UAB), Spain
Telomeric-like sequences have been found in non-terminal regions of chromosomes (so-called ITS) in a large amount of different vertebrate species. ITS have been considered the result of ancient reorganisations, alternative sites for telomere formation or markers of unstable regions. Because of the great importance of rat and other rodent species in biomedical research, the study of the distribution of ITS will contribute to the better understanding of genomic architecture and rodent evolutionary process.

Metaphasic chromosomes from fibroblasts of three foetuses from three Rattus norvegicus Sprague-Dawley females $(2 n=42)$ were obtained by standard procedures. Fluorescent in-situ hybridisation with a long synthetic (TTAGGG) ${ }^{\mathrm{n}}$ probe was performed. Only double spot signals were scored and the analysis was performed at a resolution of 238 bands/haploid genome.

A total of 305 metaphases was analysed and, as expected, all telomeres hybridised with the probe. In addition, 121 intrachromosomal loci with different hybridisation frequencies were detected along all chromosomes with the exception of chromosome RNOY. A non-random ITS distribution through the karyotype of RNO was observed. A preliminary comparative study has shown that 18 ITS were located in those chromosomal bands affected by X-radiation in $\mathrm{R}$. norvegicus; so the $75 \%$ of irradiation breakpoints coincide with ITS.

Considering ITS as origin or result of chromosomal reorganisations, the large amount of ITS found in the Rattus norvegicus karyotype would be explained by the high rate of chromosome reorganisations which characterises the evolutionary history of rodents.

\section{PO:06:58}

\section{Telomere length control in Arabidopsis plants}

\section{G. Maillet, C.I. White and M.E. Gallego}

CNRS UMR 6547, Universite Blaise Pascal, Aubière, France

Telomeres are nucleoprotein complexes at the end of eukaryotic chromosomes. They play essential roles in maintaining genomic stability by permitting full replication of chromosomes, preventing 
unwanted fusion events and protecting chromosomes ends from degradation. Telomeres are maintained at a species-specific equilibrium length. We have found that the telomere length equilibrium differs between different Arabidopsis thaliana ecotypes. To investigate the genetic regulation of telomere length, plants with differing telomere sizes were crossed and the telomere length in the hybrids analysed. For all of the hybrids, a new equilibrium in telomere length is established, intermediate between that of the two parental plants. A regulation mechanism thus shortens the longer telomeres and lengthens the shorter. To analyze the possible involvement of the Rad50, Ku80 and Lig4 proteins in the establishment of the new telomere length, Ws ecotype (long telomeres) heterozygotes for the RAD50, KU80 and LIG4 genes were crossed with wild type Col ecotype plants (short telomeres). RAD50+/ - inter-ecotype plants are unable to elongate the shorter telomeres while retaining the ability to shorter longer ones. No haplo-insufficiency was detected for the KU80 + / - inter-ecotype plants. Unexpectedly, LIG $4+/-$ interecotype plants maintain the original size of the parental telomeres. This result is the first evidence of a role for Lig4 in telomere homeostasis.

\section{PO:06:59}

\section{Telomere and TRF2/MTBP}

localization in respect to satellite DNA during cell cycle of the mouse cell line L929

\section{Kuznetsova ${ }^{1}$, A. Voronin ${ }^{1}$ and O. Podgornaya ${ }^{1}$ \\ ${ }^{1}$ Institute of Cytology RAS}

The mouse chromosomes are acrocentric, meaning that each centromere is adjacent to telomere (Tel). The location of satellite DNA, telomeres and TRF2/MTBP protein was studied during cell cycle in cell line L929. During the cell cycle, Tel underwent movement and rearrangement: most of them tend to aggregate into conglomerates in G0/G1. In prometaphase during metaphase plate formation half of Tel together with CENs arranged in circle. Clusters located between chromosome rosettes, indicating association of Tel of different chromosomes into one cluster. Decondensation of the centromeric heterochromatin consists of major satellite (maSat) and minor satellite (miSat) as well as telomeric heterochromatin went on in the middle and late $\mathrm{S}$ phase. During S/G2 transition the correspondence of Tel with the chromocenters is most apparent. In G0/G1 Tel aggregates colocalized with maSat and with miSat to lesser extend. Tel aggregates are embedded into the maSat granules at G0/G1. MTBP shift according to Tel is visible in prophase. In prometaphase, part of MTBP doesn't coincide with telomere as well. Most of Tel are colocalized with MTBP/TRF2 throughout all stages of the cell cycle though it is possible to find 1-2 telomere that are not covered with the protein. Prominent MTBP shift in respect to the Tel is visible in prophase. In prometaphase part of MTBP doesn't coincide with Tel due to the preparation of part of MTBP move to the nuclear envelope remnant in mitosis. Biochemical features of MTBP/TRF2 suggest it participation in Tel clusterization.

\section{PO:06:60}

\section{Subtelomeric duplication 7q36-qter and monosomy 13q32-qter detected by FISH in two brothers with severe mental retardation}

\author{
A. Nucaro $^{2}$, G. Crisponi ${ }^{3}$, L. Minafra ${ }^{1}$, \\ R. Rossino ${ }^{4}$ A. $\mathrm{Cao}^{2}$ and C. Cianchetti ${ }^{1}$ \\ ${ }^{1}$ Dipartimento di Neuroscienze, \\ sez. Neuropsichiatria Infantile, Universita' di \\ Cagliari, Italy; ${ }^{2}$ Istituto di Neurogenetica e \\ Neurofarmacologia, CNR, Selargius, Cagliari, \\ Italy; '3Servizio di Puericultura, Universita' di \\ Cagliari, Italy; ${ }^{4}$ Dipartimento di Scienze \\ Biomediche e Biotecnologie, Universita di \\ Cagliari, Italy
}

We describe two patients with submicroscopic duplication of 7q36.3qter and monosomy 13q32qter, detected by subtelomeric FISH probes. The patients, a 28 old man and his 21 year old sister, were referred to the Neuropsychiatric Department for severe mental retardation and dysmorphic features. 
Both had markedly delayed psychomotor development, severe mental retardation, bilateral pyramidal signs, slight ataxia. Subcortical brain atrophy and partial agenesia of corpus callosum was also present at CT scan. The dysmorphic features were: abnormal posture, microcephaly, narrow forehead, low hairline, facies significantly abnormal, long and flat face, prognathism, narrow nasal bridge, tented upper lip, dental caries, brachydactyly.

Standard cytogenetics analysis revealed an apparently normal karyotype, but a familiar history of mental retardation persuaded us of the opportunity to investigate all members of the family.

FISH with specific 7 and 13 painting probes and telomeric probes revealed a balanced translocation in the mother and in a brother of the two patients.

A son of this brother showed a severe mental retardation and dysmorphic features. Molecular and cytogenetics investigation revealed the presence of unbalance karyotype 46, XY, der(7)t $(7 ; 13)(\mathrm{q} 36 ; \mathrm{q} 32)$.(partial trisomy 13q32qter and partial monosomy 7q36qter).

\section{PO:06:61}

\section{Mental retardation: detection of subtelomeric rearrangements}

D. Parrini ${ }^{1}$, M. Betti ${ }^{1}$, G. Marseglia ${ }^{1}$, A. Nutini ${ }^{1}$, R. Ciampi ${ }^{1}$ and $F$. Torricelli ${ }^{1}$

${ }^{1}$ UO Citogenetica e Genetica, AO Careggi, Firenze, Italy

Chromosomal rearrangements involving the subtelomeric regions have been found in $7,4 \%$ of children with moderate to severe mental retardation. The detection of these rearrangements requires a FISH-based assay with a set of chromosome-specific probes, localized as close as possible to the telomere.

We have used a set of $42 \mathrm{BAC} / \mathrm{PAC}$ probes specific for the subtelomeric regions: these probes were alternative to those published by Knight et al. Every probe has been tested for sensibility and specificity. The following step was the development of a method that allows the simultaneous analysis of all the subtelomeric regions. Using data from literature, all p-arm probes were labelled with biotin-16-dUTP and detected with
avidin-CY3, all q-arm probes with digoxigenin11-dUTP and detected with antidigoxigeninFITC. Furthermore the q-arm of acrocentric and Y chromosomes were labelled simultaneasly with biotin-16-dUTP and digoxigenin-11-dUTP so that we could identify these regions as an yellow signal due to the fusion of the red signal (CY3) and the green signal (FITC).

We have used three slides per person: every slide was divided into six sections, every section was cohybridized with two or three probes differently labelled. This method was validated on negative and positive controls.

We report the results of the study of the subtelomeric regions, using the method described, in 60 children with idiopathic mental retardation.

\section{PO:06:62}

\section{How do Alliaceae maintain their telomeres? - revisited}

E. Sykorova ${ }^{1}$, K. Neplechova ${ }^{2}$, M. Sklenickova', Y. Lim $^{3}$, M. Chase ${ }^{4}$, A. Leitch ${ }^{3}$ and J. Fajkus ${ }^{1}$

${ }^{1}$ Institute of Biophysics, Czech Academy of Sciences, Kralovopolska 135, 61265 Brno, Czech Republic; ${ }^{2}$ Department of Functional Genomics and Proteomics, Masaryk University, Kotlarska 2, 61137 Brno Czech Republic; ${ }^{3}$ School of Biological Sciences, Queen Mary University of London, Mile End Road, E1 4NS London, UK; ${ }^{4}$ Jodrell Laboratory, Royal Botanic Gardens, Kew, TW9 3AB Richmond, UK

The search for missing typical plant telomeres in Asparagales revealed that this monocotyledonous plant order can be subdivided into three groups of plants differing substantially in the type of their telomeres-plant-type (e.g. orchids), humantype (e.g. asparagus), unknown type (e.g. onion). The presence of different types of telomere corresponds with phylogeny and it defines two evolutionary points where the change had happened (Sykorova et al., Proc. R. Soc. Lond. B, 2003, 270, 1893-1904). The first evolutionary point divides the families with the plant and the human type of telomeres, the second point divides the families with the human and the unknown type of telomeres (Alliaceae). To further define this 
switch point we started screening the Alliaceae family to find out where the loss of human type of telomeres had happened. Our current results of slot-blot and in situ hybridisations suggest the 'big change' happened within Allium. This finding move us closer to a solution for the mystery of onion telomeres and their maintenance. Although the previously suggested models of function of alternative onion telomeres based on satellite repeats or transposable elements could explain the mechanism of replenishment of chromosome end sequences, the other telomeric functions (e.g., the end capping) would require substantial adaptation of the protein machinery involved.

This work was supported by Leverhulme Trust, GACR (204/ 04/P105, 204/02/0027) and the institutional support (MSM143100008, Z5004920)

\section{PO:06:63}

\section{Sequence-specific control of stability of internal (TTAGGG)n repeats in mammalian cells}

\author{
N. Tomilin ${ }^{1}$, R. Krutilina ${ }^{1}$, A. Smirnova ${ }^{1}$, \\ S. Oei ${ }^{2}$ and M. Bradbury
}

${ }^{1}$ Institute of Cytology, Russian Academy of Sciences, 194064 St. Petersburg, Russia; ${ }^{2}$ Institute of Biochemistry, Free University of Berlin, 14195 Berlin, Germany; ${ }^{3}$ Department of Biological Chemistry, UC Davis School of Medicine, Davis, CA 95616, USA

A major fraction of the genome in mammals is represented by tandem and dispersed repeats which provide segments for unequal homologous recombination (UHR) leading to chromosome aberrations. UHR is known to be initiated by double-strand DNA breaks (DSBs) which formation within repeats can be suppressed by chromatin. Alternatively, DNA ends of already formed DSBs can be fixed in chromatin to prevent ectopic homologous interactions and misjoining. In some mammalian species tandem $(\mathrm{TTAGGG})^{\mathrm{n}}$ repeats are located not only in telomeres but also within internal chromosome bands and here we present evidence that internal $(\mathrm{TTAGGG})^{\mathrm{n}}$ repeats (ITRs) can be protected by telomeric protein Pin2/TRF1. This essential protein is known to be phosphorylated at its Ser-219 by ATM kinase and we have shown that inhibition of this phosphorylation by wortmannin leads to suppression of mobility of GFP-TRF1 bound to ITRs in Chinese hamster V79 cells and to an increased frequency of spontaneous chromosome aberrations (SCA) which are clustered at ITRs. Similar increase of SCA was also observed after transfection of V79 cells with the expression plasmid encoding tankyrase 1 which suppresses binding of GFP-TRF1 to ITRs. We suggest that dynamic binding of TRF1 induces formation of compact chromatin at ITRs limiting spontaneous DNA breakage by nucleases. This breakage may depend on endonuclease of L1 retrotransposons which is expressed at high level in somatic mammalian cells and stimulated by ectopic parallel pairing of $(\mathrm{TTAGGG})^{\mathrm{n}}$ repeats promoted by immobile TRF1.

\section{PO:06:64}

\section{Localization of telomere repeats in chromosomes of two species, Sorex araneus and Sorex granarius}

\section{N. Zhdanova ${ }^{1}$, T. Karamisheva ${ }^{1}$, N. Astakhova ${ }^{1}$, N. Rubtsov', J. Minina ${ }^{2}$, P. Lansdorp ${ }^{3}$ and M. Kammori}

${ }^{1}$ Institute of Cytology and Genetics of SB RAS; ${ }^{2}$ Novosibirsk State University, Russia; ${ }^{3}$ Terry Fox Laboratories, BC Cancer Research Centre, Vancouver, BC, V5Z 4E6, Canada

According to molecular clock, S.araneus and $S$ granarius diverged few tens of thousand years ago. Their karyotypes consist of practically identical chromosomal arms. In S.araneus (Novosibirsk race) these elements combines together as metacentrics, whereas karyotype of S.granarius contains a few metacentrics, de and tu, the remaining chromosomes represented by individual arms. All S.araneus telomeres exhibited hybridization signals with labeled telomere repeat probe generated by PCR. In addition, ITSs were found in the centomere of metacentics of middle size, go, hn, ik, $\mathrm{gl}, \mathrm{mp}$, and gr. In contrast, ITSs have not revealed in large, af, bc de, and small, tu, metacentrics, which are the results of more "ancient" Robertsonian 
fusion. It is appears, that in S.araneus Rb translocations were formed with the preservation of telomere repeats. Probably, they could be deleted in "ancient" metacentrics. Contrary, strong signals have been detected only in the pericentromeric regions of S.granarius acrocentrics. The use of PNA telomeric probe showed, that these regions contain up to $300 \mathrm{~kb}$ of telomeric DNA, while the telomeres of long arms $3.8 \mathrm{~kb}$ only. To study the structure of S.granarius long telomeres, we generated microlibrary from 6 copis of pericentromeric regions of a chromosomes. The microlibrary painted pericentromeric regions of the all S.granarius acrocentrics. Using two color F-FISH with telomeric PCR probe and microlibrary, we observed both cohybridization and sequential localization of two probes. We assume, that multiplication and settling of telomeric repeats simultaneously with the other repeated sequences from pericentromeric regions of a arm occurred during the formation of S.granarius karyotype.

\section{Epigenetics Symposium}

\section{L71}

\section{The biology of proteins that bind to methylated DNA}

\section{A. Bird}

University of Edinburgh, Scotland, UK

No abstract was submitted for this talk.

\section{L72}

\section{How transcriptional and epigenetic programmes are played out on an individual mammalian gene cluster during lineage commitment and differentiation}

\section{Higgs ${ }^{1}$}

${ }^{1}$ Weatherall Institute of Molecular Medicine, University of Oxford, UK

In the post-genome era a great deal of work has been focussed on understanding how DNA sequence is used to programme complex nuclear, cellular and tissue functions throughout differentiation and development. There are many approaches to these issues but we have concentrated on understanding how a single mammalian gene cluster is activated or silenced as stem cells undergo lineage commitment, differentiation and maturation. In particular we have analysed the alpha globin cluster which is expressed in a cell type- and developmental stagespecific manner in the haemopoietic system. Our studies include analysis of the transcriptional programme which accompanies globin gene activation focussing on the expression of relevant transcription factors and co-factors. Binding of these factors to the chromatin domain containing the alpha cluster has been characterised by chromatin immunoprecipitation. In addition, we have monitored the epigenetic modifications (e.g. nuclear position, timing of replication, chromatin modification, DNA methylation) that occur as the genes are activated (in erythroid cells) or silenced (e.g. in granulocytes) as haemopoiesis proceeds. Together these observations provide a uniquely well characterised model illustrating the mechanisms which regulate and memorise patterns of mammalian gene expression as stem cells undergo lineage specification, differentiation and terminal maturation.

\section{L73}

\section{Role of histone modifications in chromatin function and cancer}

\author{
T. Kouzarides ${ }^{1}$, S. Saunders ${ }^{1}$ and \\ L. Hughes-Davies ${ }^{1}$ \\ ${ }^{1}$ Wellcome/Cancer Research UK Gurdon \\ Institute. Tennis Court Road, Cambridge, UK
}

Chromatin is covalently modified at many sites within histones. Our efforts are concentrated on understanding the mechanism by which histone modifications control chromatin. In addition, since chromatin modifying enzymes are implicated in cancer we would like to know how disruption of the pathways that leads to histone modifications contribute to cancer.

Our analysis of lysine methylation has led us to examine SET domain containing proteins in the 
yeast S.pombe. We are analysing a protein SET9, which we find can methylate histone $\mathrm{H} 4$ at K20. We find that a SET9 deletion or a point mutation in the catalytic domain of SET9, shows loss of viability when exposed to DNA damaging agents such as UV and gamma irradiation. Our results indicate that SET9 (which has uncharacterised human homologues) represents a member of a new family of lysine methylases, which play a role in DNA damage response.

We are also extending our studies on the EMSY breast cancer gene. EMSY associates with the BRCA2 tumour suppressor and has a role to play in transcriptional repression and DNA repair. The EMSY protein forms a binding platform with chromatin regulators such as HP1. The domain of EMSY necessary for binding BRCA2 and HP1 is under structural analysis and the relative contribution of EMSY to transcription and DNA repair is being investigated.

\section{L74}

\section{Epigenetic reprogramming - taking a lesson from an embryo}

P. Hajkova ${ }^{1}$, P. Western ${ }^{2}$, S. Erhardt ${ }^{3}$, S. Sullivan ${ }^{1}$, F. Cesari-Weimar ${ }^{1}$, J. Brenton ${ }^{4}$ and A. Surani ${ }^{1}$

${ }^{1}$ Wellcome Trust/Cancer Research UK Gurdon Institute, Cambridge, UK; ${ }^{2}$ ARC Center for Development and Biotechnology, Murdoch Childrens Research Institute, Melbourne, Australia; ${ }^{3}$ UCSF, LBNL Berkeley, US; ${ }^{4}$ Cancer Genomics Program, Department of Oncology, University of Cambridge, Hutchison/MRC Research Centre, Cambridge, UK

Nuclear cloning experiments brought new insights into genome wide reprogramming connected with changing plasticity of genome and re-gaining of cellular pluripotency. Such reprogramming is characterised by change in global gene expression accompanied by genome wide changes of epigenetic modifications and chromatin structure. However, little is known about molecular basis of this process.

Genome reprogramming events occur naturally several times during mammalian embryonic devel- opment: In zygote, the reprogramming processes are targetted to male pronucleus thus creating an epigenetic asymmetry between parental genomes. In blastocyst, with the first wave of differentiation epigenetic reprogramming occurs in the pluripotent cells of inner cell mass (ICM) as opposed to the differentiating cells of trophectoderm (future extraembryonic tissues). The genome wide reprogramming commences later once again during the development of germline, when the developing germ cells undergo a complete erasure of all epigenetic information prior to the onset of sexual differentiation.

These naturally occuring reprogramming events are, at least to some extent, recapitulated in vitro in somatic nuclear transfer and cell fusion experiments. Search for common factors should thus shed light on molecular mechanisms underlying these complex processes.

L75

The role of SDE4 in chromosomal silencing in plants

\section{A. Herr' ${ }^{1}$ and D. Baulcombe ${ }^{1}$}

${ }^{1}$ Sainsbury Laboratory

Certain types of chromosomal silencing in plants are associated with features of RNA silencing. Small interfering RNAs (siRNAs) can be found from the silenced locus and components of the silencing machinery are required both for the production of siRNA and the silencing of the gene. Loci undergoing chromosomal silencing, such as AtSN1 (Arabidopsis Sine element 1), produce only the longer class of siRNAs (24-25nts), whereas both size classes $(21 / 22$ and $24 / 25)$ can be found in cytoplasmic-silencing systems. These size differences reflect distinct silencing pathways. Transgene silencing in Arabidopsis requires the RNA-dependent RNA-polymerase RDR6 (SDE1/SGS2) and argonaute 1 (ago1). Chromosomal silencing requires SDE4, the argonaute-like protein AGO4, and the RNAdependent RNA-polymerase RDR2. Intriguingly, mutants of the chromosomal silencing 
pathway delay the onset of GFP transgene silencing and suggest that there is cross-talk between these two pathways that enhances silencing efficiency. Map-based cloning of SDE4 suggests a model in which SDE4 helps to produce an aberrant RNA substrate that is recognized by $\mathrm{RDR} 2$.

\section{L76}

\section{Changes in the arrangement of chromosome territories in human cell clones}

\author{
D. Kähler ${ }^{1}$, J. Gao², J. Mattes², R. Eils², \\ T. Cremer ${ }^{1}$ and I. Solovei ${ }^{1}$
}

${ }^{1}$ Department of Biology II, Ludwig Maximilians University (LMU), Munich; ${ }^{2}$ iBioS, German Cancer Research Center (DKFZ), Heidelberg

The inheritance of chromosome territory arrangements (CT-A) from one cell generation to the next one has remained a controversial issue (Gerlich et al [2003] Cell 112: 751-764; Walter et al. [2003] J Cell Biol, 160: 685-697). We performed 3D-FISH experiments with sets of chromosome specific probes on human cell clones, including normal diploid fibroblasts (2-cell clones), binucleated fibroblasts, normal epithelial cells (2- and 4-cell clones) and the cancer cell lines DLD and HeLa (2-, 4-, 8-, 16-, and 32-cell clones). Nuclear image stacks were recorded with a confocal microscope and used for the 3Dreconstructions of CTs and the determination of the gravity centres of each CT. To estimate the similarity of CT-As between cells from the same clone we determined (i) the $3 \mathrm{D}$ distance differences between homologues CT gravity centres and (ii) the 'bending energy', a measure of topological alterations required to transform the CTA of one nucleus to the CT-A of another. Both approaches consistently confirmed very similar CT-As in sister nuclei of 2-cell clones or binucleated cells. For clones with larger number of cells, higher values of both parameters were found, steadily increasing from 2- to 16-cell clones. At this stage dissimilarities between CTAs reached the level observed for randomly chosen cells from the original cell population.
L77

\section{Association of glutathione S-transferase and chromosomal aberration as a means to determine occupational exposure}

\section{A. Movafagh ${ }^{1}$, F. Maleki, S. Mohamadzadehghobadloo and S. Fadaei}

${ }^{1}$ Shahid Beheshti Medical University Department Of Genetics, Evin, Tehran, Iran

It is well known that exposure of the body to ionizing radiation such as X-rays produces an extremly wide range of mutational events.The influence and status of glutathione S-transferase (GST) on the rate and frequency of chromosomal aberrations in peripheral blood lymphocytes of 50 medical radiotherapy workers over 5 years periods handling X-ray machines and 43 control individuals was subjected to our studied. GST is divided into four classes; the mu and theta showed genetic polymorphism, that involves a gene deletion. This deletion has been shown to be associated with the susceptibility to mutagen induced cytogenetic damages. GST activity in serum was estimated by improved Habig method, also the frequency of chromosomal aberrations was evaluated in blood lymphocytes by conventional Trypsin G-banding technic. Interpretation of this study yields of dicentrics acentrics followed by ring chromosomes and of total chromosomal aberrations and GST activity were significantly higher in the radiotherapy workers $\mathrm{P}=0.04$ and $\mathrm{P}=0.001$ respective compare to controls. Increase GST concentration and chromosomal damages are correlated in the present study. The mean frequencies of different chromosomal aberrations were higher in radiotherapy workers with over 10 years occupation exposure $(\mathrm{P}=0.05)$ compare to less than 10 years with similar condition. GST activity in male were more frequent $(\mathrm{P}=0.04)$ than female in radiotherapy workers. Age and sex were not found to be significant predictor for GST activity and chromosomal abnormalities in controls. Despite the limited number of blood sample the results seem to indicates an association between chromosomal aberration and GST activity, although for firm conclusion, it needs more data. 
L78

\section{Parental origin-dependent phenotype of mice transgenic on bovine satellite DNA}

\author{
I. Suchkova ${ }^{1}$, N. Slominska ${ }^{1}$ and E. Patkin ${ }^{1}$ \\ ${ }^{1}$ The Institute of Experimental Medicine \\ of RAMS, St. Petersburg, Russia
}

We produced two lines of transgenic mice harbored repeated unit $(3.8 \mathrm{~kb})$ of bovine satellite IV (Sat) in hemizygous state. Whereas Sat transmission from Sat hemizygous females did not cause the statistically significant deviation from theoretically expected $50 \%$, the transmission of Sat from male resulted in the decrease of the frequency of newborn offsprings up to $4,5 \%-13,3 \%$ in comparison with theoretically expected $50 \%$. This could be a result of our observation of abnormal and delayed embryos following implantation. The second striking effect was an appearance of mammary gland tumors in transgenic females following first lactation. In theory such effects could be ascribed to deleterious effect of Sat on one of the imprinted genes. Gbanding and FISH showed that Sat was mapped to chromosome 12 in nearest vicinity to gene Pax9, which upregulation was shown to be associated with both abnormal development and cancer. Thus, if this gene is imprinted, Sat integration resulted in loss of imprinting, and subsequent increasing of this gene product similarly to described earlier imprinted genes. Alternatively Sat could operate as transactivator of remote imprinted genes for example via attracting methylation groups from imprinted genes and/or a formation of unusual chromatin structure. Our results of heterochromatin and methylation analysis of Sat mice at least partly evidence in favor of last proposal. Sat was completely methylated in abnormal embryos and mice with tumors, and nuclei had another heterochromatin morphology in comparison with normal mice.

The work was supported by RFBR grant N 04-04-48169
PO:06:65

\section{Preferential inactivation of the parental hypermethylated $X$ chromosome in hybrid females of the genus Phyllotis (Rodentia: Muridae)}

\author{
M. Acevedo ${ }^{1}$ and L. Walker ${ }^{1}$ \\ ${ }^{1}$ Instituto de Ciencias Biomédicas, Facultad de \\ Medicina, Universidad de Chile. Santiago, Chile
}

Though is well documented that dosage compensation of X-linked genes in mammals, is achieved by random inactivation of one $\mathrm{X}$ chromosome, scarce information is available concerning this process in hybrid females. DNA methylation is one epigenetic mechanism that maintain the inactive $\mathrm{X}$ state; however, it is not clear whether inactive (iX) or active $\mathrm{X}(\mathrm{aX})$, is the hypermethylated one. To find out if the $\mathrm{X}$ chromosomes are randomly inactivated in Phyllotis hybrid females, we evaluated the methylation state of these chromosomes in males and females of the parental species, $P$. darwini and $P$. xanthopygus and in their hybrid females, assuming that $\mathrm{X}$ male methylation state corresponds to an aX chromosome.

Metaphases were treated with SPRINS technique: after HpaII digestion of non methylated Rbands, digoxygenin-labelled nucleotides were nick translated and detected with fluorescenceconjugated antibodies (Boehringer Mannheim). HpaII-SPRINS bands intensity of parental species and hybrid $\mathrm{X}$ chromosomes, were compared using an epifluorescence microscope.

The X chromosomes of both parental females showed evident differences in the intensity of their HpaII-SPRINS bands, being the strongest fluorescence of the hypomethylated one, similar to that showed by the respective male $\mathrm{X}$ chromosome. Since $\mathrm{X}$ male is genetically active, the $\mathrm{iX}$ in these females must be the hypermethylated one. The hybrid female X-chromosomes also had clear differences in their HpaII-SPRINS bands, being the darwini $\mathrm{X}$ chromosome more fluorescent than the xanthopygus $\mathrm{X}$ one. Thus, in these hybrid females the xanthopygus hypermethylated $\mathrm{X}$ chromosome is preferentially inactivated. 
PO:06:66

\section{Interchromatin compartment visualized by induced-chromatin- condensation (ICC) in living HeLa cells}

H. Albiez ${ }^{1}$, L. Schermelleh ${ }^{1}$, I. Solovei ${ }^{1}$, H. Leonhardt ${ }^{1}$ and T. Cremer ${ }^{1}$

${ }^{1}$ Department of Biology II, Ludwig Maximilians University, 80333 Munich, Germany

It was earlier demonstrated that incubation of cells in a hyperosmotic medium induces strong condensation of chromatin (Robbins et al. JCB, 40:400-416 (1970)). The effect was completely reversible after restoring physiological osmotic conditions. We have employed this approach, called Induced-Chromatin-Condensation (ICC), in living HeLa cells. ICC was obtained in less than $1 \mathrm{~min}$ following the incubation of cells in hyperosmotic medium $(525 \mathrm{mOsm} / \mathrm{l})$ and likely resulted from an increase of divalent ions in the cell interior. Transcription as well as replication was discontinued during ICC but restored as soon as the normal condensation state was reestablished. ICC was accompanied by an enlargement of the interchromatin compartment in the nucleus. In accordance with the chromosome territory-interchromatin compartment (CT-IC) model and experimental data from other groups (Cmarko et al. Histochem Cell Biol. 113:181-187 (2000)) nascent transcripts formed prior to ICC were observed at the surface of condensed chromatin lining the enlarged interchromatin compartment, while splicing speckles and nuclear bodies were retained in its interior. Cells kept in the ICC state for up to $30 \mathrm{~min}$ and then released into isotonic medium retained their viability. These features indicate that ICC provides a useful approach to study the structure-function relationships of chromatin domain surfaces and the interchromatin compartment.

PO:06:67

Spatial aspects of chromosomal inactivation during stem cell differentiation
C. Bacher ${ }^{1}$, E. Heard ${ }^{2}$ and R. Eils ${ }^{1}$

${ }^{1}$ Divisions of Intelligent Bioinformatics Systems1, German Cancer Research Center (DKFZ), 69120 Heidelberg, Germany; ${ }^{2}$ Mammalian Developmental Epigenetics Group2, UMR 218-Nuclear Dynamics and Genome Plasticity, Curie Institute-Research Section, Paris, France

$\mathrm{X}$-chromosome inactivation ensures dosage compensation between XX females and XY males. The inactivation of an entire $\mathrm{X}$-chromosome takes place while its homologue remains genetically active in the same nucleus. We are examining undifferentiated female and male mouse embryonic stem cells to investigate the spatial localisation and distribution of the $\mathrm{X}$-inactivation center (Xic) during the initiation of $\mathrm{X}$ inactivation using FISH. We have acquired 3D multicolour image sets of the fixed cell nucleus labelled with DAPI and primary transcripts at the Xic using confocal laser scanning microscopy during the first 4 days of ES cell differentiation. We used in-house developed software tools for image processing and for the quantitative analysis of the Xic signal distances to the nuclear periphery of female and male cells and the inter Xic locus distances in female cells, respectively. Positional changes of the Xic loci in female versus male cells will be presented, with a view to understanding the potential implication of nuclear localisation in the $\mathrm{X}$-inactivation process.

\section{PO:06:68}

\section{Spatio-temporal dynamics of $X$ inactivation in differentiating mouse ES cells.}

\author{
J. Chaumeil ${ }^{1}$, P. Le Baccon ${ }^{1}$ and E. Heard ${ }^{1}$ \\ ${ }^{1}$ Curie Institute, UMR 218, Mammalian \\ Developmental Epigenetics Group, Pavillon \\ Pasteur, 26 rue d'Ulm, 75248 Paris cedex 05, \\ France
}

$\mathrm{X}$-chromosome inactivation, the mechanism by which dosage compensation for the sex chromosomes between males and females is achieved in mammals, involves the stable silencing and the heterochromatinization of one of the two 
homologous $\mathrm{X}$ chromosomes during early female development. It represents a remarkable example of epigenetic gene regulation. Coating of the $\mathrm{X}$ chromosome by Xist RNA is essential for the initiation and propagation of X-inactivation. However, little is known about the mechanisms that transform this signal into transcriptional silencing. Using female embryonic stem cells as a system model, where in vitro differentiation is accompanied by $\mathrm{X}$-inactivation, we have carried out an integrated analysis of the order of appearance of early events underlying X-inactivation, showing that multiple histone $\mathrm{H} 3$ and $\mathrm{H} 4$ modifications appear immediately after Xist accumulation. This data support an early role for histones in the $\mathrm{X}$-inactivation process and suggest that they form at least part of the cellular memory of the inactive state on the $\mathrm{X}$ chromosome. We present new data showing that the transcription machinery is excluded from the Xist RNA coated $X$ chromosome, prior to the onset of histone modifications and gene silencing. We have analysed the position and dynamics during differentiation of several $\mathrm{X}$-linked genes on the $\mathrm{X}$ chromosome. We find that there may be a tight correlation between the location of genes on the $\mathrm{X}$ chromosome and their activity status during $\mathrm{X}$ inactivation.

\section{PO:06:69}

\section{Three dimensional analysis of histone methylation patterns in normal and tumor cell nuclei}

M. Cremer ${ }^{1}$, R. Zinner ${ }^{1}$, H. Albiez ${ }^{1}$, A. Peters ${ }^{5}$, T. Jenuwein ${ }^{2}$, R. Brack-Werner ${ }^{3}$, M. Speicher ${ }^{4}$, K. Pfleghaar ${ }^{4}$ and T. Cremer ${ }^{1}$

${ }^{1}$ LMU Dept. Biol. II, Munich, Germany; ${ }^{2}$ Institute of Molecular Pathology, Vienna, Austria; ${ }^{3} \mathrm{GSF}$, Institute of Molecular Virology, GSF, Munich, Germany; ${ }^{4}$ Institute of Human Genetics, Technical University and GSF, Munich, Germany; ${ }^{5}$ Friedrich Miescher Institute for Biomedical Research, Basel, Switzerland

Histone modifications represent an important epigenetic mechanism for the organization of higher order chromatin structure and gene regulation. Methylation of position-specific lysine residues in the histone $\mathrm{H} 3$ and $\mathrm{H} 4$ amino termini such as H3-K9, H3-K27 and H4-K20 has been linked with the formation of constitutive and facultative heterochromatin as well as with specifically repressed single gene loci, whereas methylation of $\mathrm{H} 3-\mathrm{K} 4, \mathrm{H} 3-\mathrm{K} 36$ and $\mathrm{H} 3-\mathrm{K} 79$ is associated with transcriptionally competent euchromatin. Using antibodies directed against different lysine methylation sites, we visualized the nuclear distribution pattern of chromatin flagged by these methylated lysines in 3D preserved nuclei of normal and malignant cell types. Optical confocal serial sections were used for a quantitative evaluation. We demonstrate distinct differences of these histone methylation patterns among nuclei of different cell types. Changes in the pattern formation were also observed during the cell cycle and after exit of the cell cycle. Our data suggest an important role of methylated histones in the reestablishment of higher order chromatin arrangements during telophase/early G1. Cell type specific histone methylation patterns are possibly causally involved in the formation of cell type specific heterochromatin compartments, composed of (peri)centromeric regions and chromosomal subregions from neighboring chromosome territories, which contain silent genes.

\section{PO:06:70}

\section{Cytological study of DNA methylation and of histone methylation state in human retinoblastoma and in blood cells}

\section{Skalníková ${ }^{1}$, E. Bártová ${ }^{2}$, S. Kozubek ${ }^{2}$ and M. Kozubek ${ }^{1}$}

${ }^{1}$ Faculty of Informatics, Masaryk University, Botanická 68a, Brno, Czech Republic; ${ }^{2}$ Institute of Biophysics, Academy of Sciences,

Královopolská 135, Brno, Czech Republic

Epigenetic silencing in eukaryotes is associated with the formation of a transcriptional repressive higher-order chromatin structure that is also characterized by methylation of DNA at cytosine nucleotides. Differential methylation of $\mathrm{CpG}$ islands has been inversely correlated with gene activity: transcriptionally active genes were found 
to be hypomethylated, whereas transcriptionally silent genes were hypermethylated (Singal R., 1999).

In our experiments, the detection of sub-nuclear localization of DNA methylated regions was performed using indirect immunofluorescence technique combined with FISH. The selected metaphase chromosomes HSA 6, 10, 11, 18, 19, 20 and 22 were determined using FISH. Sequential 5-methylcytosin rich regions were visualised using immunofluorescent labelling in different cell types. We have found, for example, that the HSA 19, 20 and 22 involved heavy methylated regions. On the other hand, HSA 18, 10, 11 and 6 were found to be unmethylated in $\mathrm{CpG}$ islands in our experiments, which is in accordance with Barbin et al., (1994). N-myc amplification detected by PCR/FISH in the retinoblastoma cell line Y79 was found to be heavy methylated in CpGislands.

Another modification linked to silencing is methylation of histone $\mathrm{H} 3$ on lysine 9 (H3-K9). Domains of euchromatin is characterised by methylation of H3-K4 (Mattei M.G., 2003). Therefore, in situ immunofluorescence, was peroformed using antibody against H3-K4. We observed orientation of methyl groups of H3-K4 into the interchromatin space in the interphase nuclei of human K-562 cells.

This work was supported by the GA CR (Grant No. 202/03/ D033) and partly also by the Min.Edu CR (Grant No. MSM143300002)

\section{PO:06:71}

Topology of the imprinted Prader-Willi/Angelman and Beckwith-Wiedemann Syndrome loci in cycling cells

\section{K. Teller ${ }^{1}$, I. Solovei ${ }^{1}$, K. Buiting ${ }^{2}$, B. Horsthemke ${ }^{2}$ and T. Cremer ${ }^{1}$}

${ }^{1}$ Department of Biology II, Ludwig Maximilians University, Munich; ${ }^{2}$ Institute of Human Genetics, University of Essen, Essen, Germany

Spatial association of oppositely imprinted regions in late S-phase, but not at other stages of the cell cycle, was reported for the
Prader-Willi/Angelman Syndrome (PWS/AS) in human cells (LaSalle and Lalande [1996] Science 272: 725-728). We re-investigated this 'kissing phenomenon' using 3D-FISH with locus-specific probes and replication labeling for a precise Sphase staging. Average relative distances (ARDs) between the two PWS/AS loci were determined in 3D confocal image stacks. In PHA-stimulated lymphocytes ARD was significantly smaller in late S-phase compared to early S-phase and quiescent lymphocytes. A corresponding decrease was found for chromosome 15 centromeres, which is located in close vicinity to both ribosomal genes on $15 \mathrm{p}$ and to the PWS/AS region on 15q11-13. In lymphoblastoid cells of a PWS patient with a complete deletion of the PWS/AS region in paternal \#15, we noted the same decrease of ARD between \#15 centromeres in late S-phase. In gorilla lymphoblastoid cells, however, where the human \#15 homolog bears the PWS/AS locus but no ribosomal genes, the ARD between the two loci remained unchanged during S-phase. Significant ARD changes during S-phase of lymphoblasts were also not detected for another imprinted locus, the Beckwith-Wiedemann region on 11q15.5. In summary, our data provide strong evidence against the postulated ãkissing phenomenonä.

\section{Plant Chromosomes Symposium}

\section{L79}

\section{Dissecting large genomes of cereals by chromosome sorting}

\author{
J. Dolezel ${ }^{1}$, M. Kubalakova ${ }^{1}$, J. Bartos ${ }^{1}$, \\ J. Safar ${ }^{1}$, J. Janda ${ }^{1}$, J. Cihalikova ${ }^{1}$, \\ P. Kovarova ${ }^{1}$ and P. Suchankova ${ }^{1}$ \\ ${ }^{1}$ Laboratory of Molecular Cytogenetics and \\ Cytometry, Institute of Experimental Botany, \\ Sokolovska 6, CZ-77200 Olomouc, Czech \\ Republic
}

Cereals provide a major portion of our diet. While some of them like rice and sorghum possess small genomes, the genomes of barley, rye and wheat are complex, consisting mainly of various classes of repetitive DNA sequences. In 
addition, the recent evolution of common wheat involved two episodes of polyploidization. These features hamper physical mapping and gene isolation. Purification of individual chromosomes by flow cytometry can greatly simplify these tasks by providing small and defined genome fractions. This lecture will review a development of the methodology and its potential for genome mapping in barley, rye and wheat. Chromosome analysis by flow cytometry permits quantitative detection of structural and numerical chromosome changes. Due to small differences in DNA content, only one chromosome can be discriminated and sorted in each of the species. However, cytogenetic stocks facilitate separation of other parts of the genomes as individual chromosomes and chromosome arms. Sorted chromosomes have been used for discovery of rare structural changes and high-resolution cytogenetic mapping using FISH. The use of sorted chromosomes for HAPPY mapping, targeted isolation of low-copy sequences, and high-throughput physical mapping of ESTs are attractive options. As millions of chromosomes with intact DNA may be sorted, construction of BAC libraries is possible. Subgenomic, chromosome-specific and chromosome arm-specific BAC libraries have already been constructed and represent unique resources for wheat genomics.

This work has been supported by the Grant Agency of the Czech Republic (grants 522/03/0354 and 521/04/0607), and the Ministry of Agriculture of the Czech Republic (grant QC1336).

\section{L80}

\section{Speciation-related chromosomal} repatterning in a small peripheral plant population: assumption from rDNA clusters variability.

\section{O. Raskina ${ }^{1}$, E. Nevo ${ }^{1}$ and A. Belyayev ${ }^{1}$ \\ ${ }^{1}$ Institute of Evolution, University of Haifa, Israel}

We present a particular case of the chromosomal repatterning in connection with sympatric speciation in the Sitopsis section of genus Aegilops (Poaceae, Monocotyledones). Two small, peripheral, isolated, wild populations of annual cross-pollinated Ae. speltoides and annual selfpollinated Ae. sharonensis are located 30 meters apart on different soil types. Despite the close proximity of the two species and their close relatedness, no mixed colonies are known due to seasonal, ecological, and biological isolation. Comparative molecular cytogenetic analysis based on intra-population variability of rDNA chromosomal patterns of individual Ae. speltoides genotypes revealed directional and rapid process of chromosomal repatterning. The mechanisms for repatterning are heterologous recombination and/or transposable elements mediated rDNA transfer. FISH experiments revealed the inter- and intra-chromosomal transfer of 5S rDNA in complex with En/Spm elements. In its turn, arising of a new rDNA site may induce a number of intra-genomic events consequent from heterologous synapses and recombination between chromosomes that carry extended rDNA arrays. The process is in an equilibrium state ö chromosomal mutations can arise de novo and can be eliminated. Analysis of the progeny of the investigated genotypes testifies that inheritance of de novo rDNA sites happens frequently. Stabilization of new rDNA clusters is achieved by homozygotization. Consequently, several modified genomic forms intermediate between Ae. speltoides and Ae. sharonensis permanently arise in studied wild populations, that make it possible to recognize Ae. sharonensis as a derivative species of Ae. speltoides, as well as to propose rapidness and canalization of the speciation process in Sitopsis species.

\section{L81}

\section{Variable organization of the terminal regions in rye chromosomes}

\section{O. Alkhimova ${ }^{1}$ and A. Vershinin ${ }^{2}$}

${ }^{1}$ Institute of Molecular Biology and Genetics, Kiev 03143, Ukraine; ${ }^{2}$ Institute of Cytology and Genetics, Novosibirsk 630090, Russia

Terminal regions, telomeres and subtelomeres, of rye chromosomes consist mostly of highly repetitive tandemly organized DNA sequences. Little is known about internal structure of tandem arrays, 
including total array size and the pattern of monomer distribution within arrays. We addressed these issues for telomere associated heterochromatin of individual rye chromosomes, using wheat-rye addition lines and rye specific DNA probes. FISH on meiotic rye chromosomes revealed a specific mosaic arrangement of domains for each chromosome arm where different tandem repetitive families localized predominately without obvious tendency in domains order, size and spacer distribution. Each wheatrye additional line studied by pulse field gel electrophoresis produced a chromosome-specific overall blot hybridization display indicating a unique large-scale organization of the tandem repeat arrays on each rye chromosome. The FISH signals on DNA fibers showed the multiple patterns of co-linear monomers arrangement of repetitive families as well as of authentic telomeric probe from Arabidopsis. The majority of the arrays consisted of the monomers of different families in various alterations separated by spacers. The primary structure of some spacer sequences revealed the scrambled regions of similarity to various known repetitive elements indicating the concerted action of several DNA break-repair mechanisms. This level of complexity in the long-range organization of tandem arrays has not been previously reported for any plant species. The various patterns of the tandem arrays internal structure is likely to have resulted from the evolutionary interplay, array homogenization and the generation of heterogeneity mediated by double-strand breaks (DSBs) and associated repair mechanisms.

\section{L82}

\section{Dynamic changes of histone $\mathrm{H4}$ acetylation in mitotic cells of barley}

\section{T. Wako ${ }^{1}$ and K. Fukui ${ }^{2}$}

\author{
${ }^{1}$ National Institute of Agrobiological Sciences \\ and ${ }^{2}$ Graduate School of Engineering, \\ Osaka University, Japan
}

Covalent modifications in nucleosome histones play various roles in cellular functions. One of the most important modifications is histone acetylation at lysine residues which occurs, in case of histone $\mathrm{H} 4$, at four lysine residues, $\mathrm{K} 5, \mathrm{~K} 8, \mathrm{~K} 12$ and K16. Dynamic changes of histone acetylation throughout mitosis and its relationships to mitotic chromosome were analyzed using a threedimensional immunofluorescent method to facilitate a comprehensive understanding of histone H4 acetylation. Basically two patterns of dynamics were revealed according to the region specific acetylations and their stages in mitotic barley cells. One of the pairwise acetylation at K5/K12 during mitosis is shown in the nucleolar organizing regions (NORs). The NORs escape from full condensation when the whole chromosomes are fully condensed, pairwise acetylation of $\mathrm{K} 5 / \mathrm{K} 12$ in $\mathrm{H} 4$ at the NORs should serve to avoid tight condensation as found in the other chromosomal regions. The second pairwise acetylation involves $\mathrm{K} 8 / \mathrm{K} 16$, which were observed in the distal regions of chromosomes, although their dynamics was not identical. The distal regions are corresponding to gene-rich regions, thus this pairwise acetylation should involve in transcriptional regulation. In addition, the pairwise acetylation of $\mathrm{K} 5 / \mathrm{K} 12$ is involved in acetylation of centromeric regions together with $\mathrm{K} 8$ acetylation. These findings indicate that combinations of acetylation correlating chromosome structure seem to have functional roles in centromere function, and transcription in the NORs and the gene-rich regions. Therefore, it is concluded that the combinations of histone $\mathrm{H} 4$ acetylation could serve as novel histone codes for chromosome structure and related functions during mitosis.

\section{L83}

\section{Dynamic analyses of Aurora kinases in plants}

S. Matsunaga ${ }^{1}$, A. Kawabe ${ }^{1}$, K. Nakagawa ${ }^{1}$, A. Yoneda ${ }^{2}$, D. Kurihara ${ }^{1}$, S. Uchiyama ${ }^{1}$, S. Hasezawa ${ }^{2}$ and K. Fukui ${ }^{1}$

${ }^{1}$ Department of Biotechnology, Graduate School of Engineering, Osaka University, 2-1 Yamadaoka, Suita, Osaka 565-0871, Japan; ${ }^{2}$ Department of Integrated Biosciences, Graduate School of Frontier Sciences, University of Tokyo, 5-1-5 Kashiwanoha, Kashiwa, Chiba 277-8562, Japan 
Aurora kinases are serine/threonine protein kinases with essential roles in cell division through eukaryotes. They regulate the interaction between cytoskelton and chromosomes at the kinetochore, the spindle assembly and the cytokinesis. We identified three Aurora kinases in Arabidopsis thaliana, AtARK1, AtARK2 and AtARK3. The kinase domain of AtARK1 shares 95\% and 63\% amino acid identity with AtARK2 and AtARK3, respectively. There are two transcriptional forms of AtARK2. A deletion type of transcript (AtARK2S) lacks the fourth exon that encodes a part of kinase domain compared to complete type (AtARK2L). These two transcripts were detected in almost all tissues but the AtARK2S expressed very low amount. The AtARK2S expressed relatively higher in roots, leaves and stems. To reveal the localization of AtARKs during plant mitosis, dynamic analyses was performed using GFPfused proteins in tobacco BY-2 cells. The localization of AtARK proteins was classified into two classes. One class showed subcellular localization of mitotic spindles during cell division. The other class showed the localization on chromosomes. The localization of AtARK proteins was different from that of animal Aurora kinases, reflecting the difference in mitotic mechanisms between plant and animal.

\section{L84}

\section{Sister chromatid non-cohesion is common in Arabidopsis thaliana interphase nuclei}

\section{Schubert, M. Klatte ${ }^{1}$, A. Pecinka ${ }^{1}$ and I. Schubert ${ }^{1}$}

${ }^{1}$ Institute of Plant Genetics and Crop Plant Research (IPK), Corrensstrasse 3, D-06466 Gatersleben, Germany

It is widely assumed that sister chromatids are cohesed from replication in $\mathrm{S}$ phase until the beginning of anaphase. However, FISH with individual BACs $(\sim 100 \mathrm{~kb})$ from different positions along chromosomes 1 and 4 to $4 \mathrm{C}$ root and leaf nuclei yielded frequently more than two hybridization signals indicating non-cohesion of sister chromatids in $32.5 \%$ to $59.6 \%$ of nuclei. In
$8 \mathrm{C}, 16 \mathrm{C}$ and $32 \mathrm{C}$ leaf nuclei up to $100 \%$ of nuclei revealed sister chromatid separation. Simultaneous FISH with BACs from different positions along chromosome 1 has shown that sister chromatid separation can be punctual and is more frequent in mid arm compared to terminal or pericentromeric positions. The high frequency of spatially separated sister chromatid arms in nuclei with $4 \mathrm{C}$ or higher DNA content suggests that sister chromatid cohesion along the entire chromosome arms is not obligatory at least in differentiated cells.

Differential labelling of chromosome 1 top arm territory and BAC T2P11 therein revealed in $5.3 \%$ of $4 \mathrm{C}$ leaf nuclei separated sister chromatid arm territories and in $38.1 \%$ punctual non-c ohesion at the segment corresponding to the T2P11 sequence. In $12.8 \%$ of nuclei $(\mathrm{n}=359)$ BAC signals were found to be looped out from the arm territory.

\section{L85}

\section{Quantum speciation in the wild Aegilops species on the base of chromosomal rearrangements directional selectivity}

\author{
A. Belyayev ${ }^{1}$, O. Raskina ${ }^{1}$ and E. Nevo ${ }^{1}$ \\ ${ }^{1}$ Institute of Evolution, University of Haifa, Israel
}

The evolution of diploid and polyploid wheats is connected with significant remodeling of rDNA sites. In light of this, we explored deviations in the distribution of $5 \mathrm{~S}$ and $45 \mathrm{~S}$ rDNA sites in natural populations of diploid Sitopsis species of the genus Aegilops (Ae. speltoides, Ae. longissima, Ae. searsii, Ae. sharonensis, and Ae. bicornis). Chromosomal rearrangements involving $5 \mathrm{~S}$ and 45S rDNA sites as well as long arms and the satellites of chromosomes 1,5 , and 6 were determined in homo- and heterozygotes in Ae. speltoides populations. These rearrangements are permanent for different populations, and genotypes with rearranged rDNA patterns are similar to other sitopsis species. The progeny of plants carrying modified rDNA patterns mainly followed parental genotypes after selfing. However, we regularly observed both the emergence of new 
mobile rDNA sites as well as genome "normalization", i.e., elimination of the additional rDNA sites. Modified rDNA clusters have been stabilized in populations in two alternative ways: (i) by selfing or crossing with similar genotypes, and (ii) by non-Mendelian epigenetic mechanisms of En/Spm mediated intra-genomic transfer of rDNA. Directional selectivity of chromosomal rearrangements in wild populations and finding intermediates between Ae. speltoides and other Sitopsis species genotypes testify existence of certain evolutionary channels leading from the plastic Ae. speltoides genome to other Sitopsis species.

\section{L86}

\section{Molecular cytogenetics and plant genome organization}

\section{P. Heslop-Harrison ${ }^{1}$}

${ }^{1}$ Department of Biology, University of Leicester, LE1 7RH, UK

Plant genomes consist of genes (which have many similarities over a wide range of species), low copy and regulatory sequences, and repetitive DNA sequences. The repetitive sequences include highly conserved units - such as the very abundant retroelements which are found in virtually all organisms. I will discuss the evidence for the major classes of retroelements including pararetroviruses in plants, and their evolutionary mechanisms in the context of chromosomal distribution. I will also discuss the nature and evolution of tandemly repetitive DNA, which is rapidly evolution and often specific to one genome or group of genomes.

References to our work are available on www.molecularcytogenetics.com, and I thank CREST, British Council, IAEATropical Fruits and EU FP VI for support of aspects of the work.

\section{L87}

Histone $\mathrm{H3}$ phosphorylation and chromosome dynamics in plants
A. Houben ${ }^{1}$ and D. Demidov ${ }^{1}$

${ }^{1}$ Institute of Plant Genetics and Crop Plant Research (IPK), 06466 Gatersleben, Germany

Post-translational modifications of histone $\mathrm{H} 3$ have been linked to chromatin dynamics and the chromosome segregation for a number of eukaryotes. Using specific antibodies we have analysed the spatio-temporal distributions of phosphorylated $\mathrm{H} 3$ at positions serine 10, 28 and threonine 11 during mitosis and meiosis. Interestingly, although histone $\mathrm{H} 3$ and most of its posttranslational modifications are highly conserved, a number of significant differences exist between animals and plants. The temporal and spatial pattern of histone $\mathrm{H} 3$ phosphorylation at serine 28 and serine 10 is similar in plants and the cell cycle-dependent phosphorylation at both serine positions are likely to be involved in the same process, such as sister chromatid cohesion but differs between mono- and polycentric chromosomes. In contrast, phosphorylation at threonine 11 temporally correlates with condensation of mitotic and meiotic chromosomes in plant cells.

In order to gain more insights into the function of histone $\mathrm{H} 3$ phosphorylation we have identified Aurora-like kinases in Arabidopsis thaliana. Aurora/Ipl1 kinases are characterized for yeast, Drosophila, Caenorhabditis elegans and a number of vertebrates. In this species the function of these kinases is closely linked to microtubule dynamics, chromosome segregation and histone $\mathrm{H} 3$ phosphorylation. To analyse whether the modulation of kinase activity influences the phosphorylation status of histone $\mathrm{H} 3$ and segregation behaviour of chromosomes, overexpression/inactivation studies of AtAurora-kinases were performed. Expression patterns, in vitro kinase assay and protein localization analyses suggest that AtAurora kinases are involved in the cell-cycle regulated phosphorylation of histone $\mathrm{H} 3$.

\section{L88}

Genetic and epigenetic consequences of a paracentric inversion in Arabidopsis thaliana

P. Fransz ${ }^{1}$, G. Linc ${ }^{1}$, H. Ali ${ }^{2}$, I. Schubert ${ }^{2}$, J. Wennekes ${ }^{3}$, H. De Jong ${ }^{3}$, M. Koornneef ${ }^{3}$, J. Peters ${ }^{4}$ and T. Gerats ${ }^{4}$ 
${ }^{1}$ University of Amsterdam, The Netherlands;

${ }^{2}$ IPK Gatersleben, Germany; ${ }^{3}$ Wageningen University, The Netherlands; ${ }^{4}$ University of Nijmegen, The Netherlands

Heterochromatic knobs are microscopically visible as islands of compact chromatin on chromosome arms. The model plant Arabidopsis has a unique $700 \mathrm{Mb}$ heterochromatic knob, hk4S, which is completely sequenced. So far, only two accessions, Columbia $(\mathrm{Col})$ and Wassilevskija (Ws), contain this heterochromatic island. We demonstrated by FISH that hk4S originated from the pericentric heterochromatin after a paracentric inversion event. Here we investigated the extent of the inversion and the genetic and epigenetic consequences. We applied FISH with BACs that span the region from the knob to the pericentric heterochromatin and compared the knob accessions, Col and Ws, with knobless accessions, Ler and C24. The FISH data confirm a paracentric inversion event in chromosome arm $4 \mathrm{~S}$. The inversion is flanked by the BAC regions F9H3 and C6L9 at the distal border of the knob and the pericentric heterochromatin, respectively. The inversion covers more than $1200 \mathrm{~kb}$ and includes euchromatic and heterochromatic segments. By fiber-FISH we could finemap the distal breakpoint of the inversion at the proximal side of $\mathrm{F} 9 \mathrm{H} 3$. The results indicate that the paracentric inversion is followed by a $20 \mathrm{~kb}$ deletion in the $\mathrm{F} 9 \mathrm{H} 3$ region of the knob accessions, and led to a decrease in genetic recombination events. Moreover, AFLP analysis of the inversed region showed reduced polymorphism between $\mathrm{Col}$ and Ws compared to flanking regions, suggesting that the inversed region has been genetically stable since the inversion event. Finally, the inversion has altered the heterochromatin status of some segments and created new borders between heterochromatin and euchromatin.

\section{L89}

\section{Differential localization of the centromere-specific proteins in Arabidopsis thaliana}

${ }^{1}$ Research Institute for Bioresources, Okayama University, Kurashiki 710-0046, Japan.; ${ }^{2}$ Core Research for Evolutionary Science and Technology, Japan Science and Technology, Kawaguchi 332-0012, Japan

The 180-bp family of tandem repetitive sequences, which comprises the major centromeric satellite in Arabidopsis thaliana, is thought to play important roles in kinetochore assembly. However, no direct evidence supporting this assertion has been obtained, to date. In the cultured cells of $A$. thaliana, drastic changes in chromosome number and structure have occurred; nevertheless no chromosomes without 180-bp repeats were found. To assess the centromere activities of the 180-bp repeats, we performed indirect fluorescence immunolabeling with antibodies against AtCENP-A or HTR12 (Arabidopsis centromeric histone H3 variant) and AtCENP-C (Arabidopsis CENP-C homologue). The immunosignals from those antibodies appeared on all sites of the 180-bp repeats detected by fluorescence in situ hybridization, except a number of minor sites between the two major sites on putative dicentric chromosomes. In addition to these colocalizations of the antibodies with the 180bp repeats, anaphase-bridge formation by the putative dicentric chromosomes carrying two 180-bp sites indicated the close relationship between the 180-bp repeats and centromere activity. However, some of the 180-bp repeat clusters, particularly those that were long or stretched at interphase, were not fully covered with the signals from antiHTR12 or AtCENP-C. Chromatin fiber immunolabeling clearly revealed that the centromeric proteins localize only at the knobs on the extended chromatin fibers, which form a limited part of the continuous 180-bp clusters. The chromatin immunoprecipitation assay using anti-AtCENP-A suggested that this sort of differential localization of the centromeric proteins is caused by variation in DNA sequences of the 180 -bp repeats.

\section{L90}

The centromere of rice chromosome 8 spans $\sim 750 \mathrm{~kb}$ of DNA and contains active genes

J. Jiang ${ }^{1}$ 
${ }^{1}$ Department of Horticulture, University of Wisconsin-Madison, Madison, WI 53706, USA

The centromeres in most multicellular eukaryotes are embedded within cytologically distinctive heterochromatin and are associated with long tracts of repetitive DNA. Thus centromeric regions typically do not contain active genes and are associated with suppression of genetic recombination. Centromeres often contain megabase-sized array of satellite DNA, which resist mapping, cloning and sequencing. Rice centromeres contain a 155-bp satellite repeat CentO that varies from $\sim 60 \mathrm{~kb}$ to $2 \mathrm{Mb}$ among different centromeres. We sequenced a $\sim 1.5 \mathrm{Mb}$ DNA contig that encompasses the cytologically defined centromere of rice chromosome 8 (CEN8). The CEN8 sequence was successfully determined largely because of its unusually low abundance of the CentO satellite. A $\sim 740 \mathrm{~kb}$ region within CEN8 binds rice CenH3, the centromere-specific H3 histone. Surprisingly, thirteen predicted and at least three active genes are interspersed in the CenH3-binding domain. The retrotransposons located within and outside of the CenH3-binding domain show similar ages and structural dynamics. Thus CEN8 shows a different DNA structure from the centromeres of most other model complex eukaryotes. We propose that CEN8 may represent an intermediate stage as centromeres evolve from genic regions, as in human neocentromeres, to fully mature centromeres that accumulate megabases of homogeneous satellite arrays.

\section{PO:06:72}

\section{En/Spm-like transposons in wild Triticeae species: preference to a certain chromosomal regions}

\author{
A. Altinkut ${ }^{1}$, O. Raskina ${ }^{2}$, E. Nevo $^{2}$ and \\ A. Belyayev ${ }^{2}$ \\ ${ }^{1}$ Tubitak, Research Institute for Genetic \\ Engineering and Biotechnology, 41470, Gebze, \\ Kocaeli, Turkey; ${ }^{2}$ Institute of Evolution, \\ University of Haifa, Mount Carmel, Haifa, 31905, \\ Israel
}

Enhancer/Suppressor-mutator (En/Spm) transposons belong to class II of transposable elements (TE). They can be activated several times in one generation and there is evidence that duplication and insertion of transposons can occur at low levels in somatic and pre-germinal cells, but increases during meiosis. En/Spm transposons are known to be linked with genes and their expression, and transposition is controlled by interacting auto regulatory and/or epigenetic mechanisms and is, therefore, relatively independent of their chromosomal localization. Indeed FISH analysis of $\mathrm{En} / \mathrm{Spm}$ transposons distribution on mitotic and meiotic chromosomes of four wild Triticeae species namely Triticum monococcum, Triticum urartu, Aegilops speltoides and Hordeum spontaneum reveal mobile clustering of En/Spm through both somatic and meiotic chromosomes. Nevertheless, our data indicate that there are certain preferences in En/Spm transposons insertions. The majority of the huge En/Spm clusters are associated with 5S rDNA sites while no association with $45 \mathrm{~S}$ rDNA sites has been detected. Second peculiarity is that this type of TE predominantly associated in mitosis and meiosis with mobile 5S rDNA sites on A and $\mathrm{B}$ chromosomes, and part of de novo arisen sites can be inherited. These data testify distinct role of En/Spm transposons in the ongoing process of intraspecific chromosomal repatterning, which, in its turn, could lead to new character combinations and therefore to novel genetic lineages-the main source for the speciation process.

\section{PO:06:73}

\section{Chromosomal mapping of $\mathrm{N}$-genome specific DNA sequences in the Triticeae}

\section{K. Anamthawat-Jonsson ${ }^{1}$ \\ ${ }^{1}$ University of Iceland, Department of Biology, Askja, Sturlugata 7, Reykjavik 101, Iceland}

$\mathrm{N}$-genome specific sequences (pLmIs1,pLmIs44 and pLmIs54) were isolated from two species of Leymus Hochst. (Poaceae: Triticeae): tetraploid northern American/Pacific L. mollis (Trin.) 
Pilger, and octoploid northern European $L$. arenarius (L.) Hochst. They are retroelement-like sequences and are found essentially in Leymus and Psathyrostachys Nevski. Fluorescence in situ hybridization (FISH) experiment show dispersed pattern of sequence distribution on all Leymus chromosomes, except at centromeres, telomeres and NORs as expected. Southern genomic in situ hybridization experiments show that both genera contain only the N-genome of Psathyrostachys, no other Triticeae genomes are involved, and therefore the polyploid genus Leymus can only be autopolyploid or segmental allopolyploid consisting of some variation of the basic N-genome. This molecular and cytogenetic evidence clearly support the taxonomic integration of the diploid genus Psathyrostachys into Leymus. The polymorphism within these sequences is found to be informative and reflecting phylogenetic relationships among the $\mathrm{N}$-genome species investigated. We are now investigating genomic and genetic relationships among species of Leymus from both Eurasia and N-America, in comparison to species of Psathyrostachys and a number of related taxa, using mainly the $\mathrm{N}$-genome specific sequences isolated here. The $\mathrm{N}$-genome species examined so far form distinct cluster-group separated from other Triticeae genomes. The tetraploid wood barley Hordelymus europaeus (Jessen) Harz is now shown to be one of the N-genome species, presumably Leymus. The paper will also describe molecular distribution, and mobility, of the gypsy-like retroelement pLmIs44 in synthesized hybrids between wheat (Triticum L.) and Leymus.

\section{PO:06:74}

\section{Chromosome numbers of some Polish liverworts from genus Aneura, Pellia and Conocephalum (Hepaticeae)}

\section{E. Chudzinska ${ }^{1}$}

${ }^{1}$ Adam Mickiewicz University, Poznan, Poland

Material on studies in bryophyte cytology had been relatively limited in Central Europe. Furthermore only about 70 percent of species have a designated chromosome number despite the fact that karyological analysis are very important in phylogenetic and systematic studies. The results in the present study are based on twelve hepatics species collected in Poland. Chromosome numbers for five species had not been previously counted. A comparison between chromosomes of three sibling species was made. Different techniques were used to prepare gametophytic mitosis for examination in all these species. For the small chromosomes (Conocephalum, Nardia, Cephalozia), the best results were obtained with the DAPI staining method. Chromosomes with big heterochromatin fragments (e.g. Pellia, Aneura) were analyzed and compared using the Giemsa $\mathrm{C}$ banding method. This method allows us to distinguish chromosomes between sibling species from the Pellia epiphylla complex and to confirm that they are parental species of Pellia borealis-tetraploid liverwort originated through allopolyploidysation. In conclusion, different cytological techniques are capable of providing answers to many important questions, such as the origin of polyploid species or taxonomic status of cryptic species.

\section{PO:06:75}

\section{The large-scale organization of Beta centromeres}

\author{
D. Dechyeva ${ }^{1}$, B. Weber ${ }^{1}$, C. Dombrowski ${ }^{1}$, \\ T. Wenke ${ }^{1}$, G. Menzel $^{1}$ and T. Schmidt ${ }^{1}$ \\ ${ }^{1}$ Institute of Botany, Technical University of \\ Dresden, D-01062 Dresden, Germany
}

The DNA composition of plant centromeres displays a high degree of variation, however, their long-range sequence organization apparently follows similar structural rules. Most plant centromeres consist predominantly of highly repetitive DNA sequences. Characteristic sequence landmarks in the centromeric heterochromatin are rare, making the molecular analysis of these regions very difficult.

To analyze a single centromere, we used monosomic chromosome fragments (minichromosomes) found in interspecific Beta hybrids such as PRO1 and PAT2. These minichromosomes originating from the wild beet Beta procumbens and Beta patellaris, respectively, are stably maintained 
in sugar beet (Beta vulgaris) and provide an experimental system towards the molecular isolation of individual plant centromeres.

Beta centromeres have a complex structure and consist of variable repetitive sequences, in particular satellite DNA and retrotransposons. By BAC sequencing and high-resolution FISH on pachytene chromosomes and extended DNA fibres we analyzed the centromeric DNA of the PRO1 and PAT2 minichromosomes.

Centromeric BACs contain highly amplified Ty3-gypsy retrotransposons as demonstrated by the existence of conserved functional domains and the presence of LTRs (Long Terminal Repeats). Most copies of the Ty3-gypsy retrotransposons Beetle1 (6738 bp) are nested, rearranged or inserted into Beetle2 (6684 bp).

The arrays pTS5 and pTS4.1 satellite of PRO1 have a length of approximately $350 \mathrm{~kb}$ and $300 \mathrm{~kb}$, respectively. Assuming that pTS5 is the functional centromeric satellite, the PAT2 centromere is contained in $47-50 \mathrm{~kb}$ as shown by restriction analysis and might be represented by a single BAC clone.

This work is funded by grants from the BMBF (BioFuture grant 0311860).

\section{PO:06:76}

\section{Developing a standard karyotype for loblolly pine using FISH and AT-rich banding}

\author{
N. Faridi ${ }^{1}$, C. Nelson ${ }^{2}$ and T. Kubisiak ${ }^{2}$
}

${ }^{1}$ USDA Forest Service, Southern Institute of Forest Genetics, Forest Tree Molecular Cytogenetics Lab, 1042 Agronomy Rd., College Station, TX77843, USA; ${ }^{2}$ USDA Forest Service, Southern Institute of Forest Genetics, Harrison Experimental Forest, Saucier, MS39574, USA

A standard karyotype has been developed for loblolly pine based on fluorescent in situ hybridization (FISH) using various labeled probes such as 18S-28S rDNA, 5S rDNA, Arabidopsis-like telomere repeat, and DAPI positive bands. Somatic chromosomes were prepared from colchicine treated root meristems using an enzymatic digestion technique. Loblolly pine has 12 pairs of chromosomes, 11 of which were previously indistinguishable based solely on size. Combinations of different probes and DAPI stain have made it possible to uniquely discriminate among all 12 pairs of loblolly pine chromosomes. A similar karyotype was developed for slash pine for direct comparison with loblolly pine as well as for comparison with a previously published slash pine karyotype. This has allowed us to identify homologous chromosomes between loblolly and slash pines. Seven intercalary and ten proximal $18 \mathrm{~S}$ rDNA sites were identified. All intercalary $18 \mathrm{~S}$ rDNA sites appeared to be major sites. Chromosome 1, contained an intercalary major 5S rDNA site. Two other independent metacentric pairs of chromosomes showed distal minor 5S rDNA sites. All but one chromosome showed DAPI positive bands. Telomere repeat signals were observed, as expected, towards the end of each chromosomal arm. With the exception of one or possibly two pairs, minor to major telomere signals were observed near or around the centromeres. Statistical analysis on chromosome arm lengths (short and long), and intercalary telomeric and rDNA positions has been carried out. Efforts are currently underway to combine our linkage maps with the karyotypes to provide a fully integrated cyto-molecular map.

\section{PO:06:77}

\section{Molecular cytotaxonomic study of a new model grass, Brachypodium distachyon}

\section{R. Hasterok ${ }^{1}$, J. Draper ${ }^{2}$ and G. Jenkins ${ }^{2}$}

${ }^{1}$ Department of Plant Anatomy and Cytology, University of Silesia, Jagiellonska 28, 40-032

Katowice, Poland; ${ }^{2}$ Institute of Biological Sciences, Edward Llwyd Building, University of Wales, Aberystwyth, Penglais, Aberystwyth, Ceredigion, SY23 3DA, UK

Brachypodium distachyon has been proposed as an alternative model organism to rice, with potential for functional genomic analysis of temperate forage grasses and cereals. Its major advantages are favourable phylogenetic position as a 'bridge species' between tropical 
and temperate cereals, a genome as small and as economical as that of $A$. thaliana, short life cycle, undemanding growth requirements, inbreeding habit and many other useful features. On taxonomic grounds Brachypodium is a genus of widely distributed temperate grasses with relatively few species, two features indicating a very ancient origin. B. distachyon has a somatic chromosome number of 10 , but ecotypes have been identified with multiples of this number. These prompted taxonomists to suggest that this species had evolved a polyploid series based upon $2 \mathrm{n}=2 \mathrm{x}=10$, and that ecotypes which deviate from multiples of 10 are aneuploid. In order to confirm the genomic constitution of five putative polyploid ecotypes, we undertook molecular cytogenetic analyses using fluorescence in situ hybridization (FISH) with total genomic (GISH) and ribosomal DNA probes. The results obtained challenge the assumption that the series evolved simply by chromosome doubling and suggest that the ecotypes with 30 chromosomes are in fact allotetraploids arising from interspecific hybridization between $B$. distachyon $(2 \mathrm{n}=2 \mathrm{x}=10)$ and another Brachypodium species $(2 \mathrm{n}=2 \mathrm{x}=20)$ with a genome resembling that of a related diploid species, B. sylvaticum.

The authors acknowledge financial support from the Royal Society (Joint Project 2001-2002 to R.H. and G.J.) and the Biotechnology and Biological Sciences Research Council (ISIS award to R.H. and J.D. 2003-2004).

\section{PO:06:78}

\section{A bacterial artificial chromosome (BAC) library for a new model grass, Brachypodium distachyon}

R. Hasterok ${ }^{1}$, A. Marasek ${ }^{2}$, G. Jenkins ${ }^{3}$, I. Donnison ${ }^{4}$, A. Thomas ${ }^{4}$, I. King ${ }^{4}$ and J. Draper ${ }^{3}$

${ }^{1}$ Department of Plant Anatomy and Cytology, University of Silesia, Jagiellonska 28, 40-032 Katowice, Poland; ${ }^{2}$ Department of Biotechnology of Ornamental Plants, Research Institute of Pomology and Floriculture, 96-100 Skierniewice, Poland; ${ }^{3}$ Institute of Biological Sciences, Edward Llwyd Building, University of Wales,

Aberystwyth, Penglais, Aberystwyth, Ceredigion,
SY23 3DA, UK; ${ }^{4}$ Institute of Grassland \& Environmental Research, Plas Gogerddan, Aberystwyth, Ceredigion SY23 3EB, UK

Brachypodium distachyon is a ubiquitous grass species of ancient origin that occupies a distinctive phylogenetic position before the radiation of the temperate cereals and grasses. Its highly desirable biological attributes such as an exceptionally compact genome, inbreeding habit and short generation time, are being exploited in a new functional genomic initiative to use $B$. distachyon as a 'bridge' species to gain access to important syntenic regions of the genomes of less tractable relatives such as wheat. As part of the development of a functional genomics toolkit for this species, a BAC library has been constructed. The library contains about 8,700 clones, with an average size of about $80 \mathrm{kbp}$, giving about 4.5 times genome coverage. Regions in the rice genome database have been identified that are syntenic to loci controlling agronomically important traits in wheat, such as disease resistance and heading date. Primers of conserved regions have been designed and used to identify by PCR BACs containing orthologous regions in $B$. distachyon, with a view to demonstrating the utility of this species for gene isolation. The level of microsynteny will be assayed by sequencing. BACs containing low copy or unique sequences are also being anchored by FISH to the 10 chromosome arms of this species, and 'landed' on somatic chromosomes of wheat, rye and rice, in order to reconstruct the archetypal grass genome.

The authors acknowledge financial support from the Royal Society (Joint Project 2001-2002 to R.H. and G.J.) and the BBSRC (ISIS award to R.H. and J.D. 2003-2004).

\section{PO:06:79}

Genotoxic potential of soil of Amritsar using chromosomal aberration assay in Allium cepa

\section{J. Katnoria ${ }^{1}$, A. Nagpal ${ }^{1}$ and R. Bhardwaj ${ }^{1}$}

${ }^{1}$ Dept. of Botanical and Environmental Sciences, Guru Nanak Dev University, Amritsar, Punjab, India 
The environmental contamination with heavy metals has increased drastically in recent years. Soils polluted with heavy metals can cause phytotoxicity and exhibit impaired microbial activities. Apart from being highly toxic to biological systems, the subtle danger of presence of heavy metals in soil lies in their being genotoxic. As plant bioassays are most sensitive in detecting genotoxicity of environmental agents and serve as the first alert for the presence of environmental hazards, the present study was planned to evaluate the genotoxic potential of extracts of soil samples collected from different agricultural fields of Amritsar $\left(31^{\circ} 37^{\prime} \mathrm{N}\right.$ and $\left.74^{\circ} 52^{\prime} \mathrm{E}\right)$, employing Chromosome Aberration Assay in Allium cepa. This assay was validated in 1991, by the International Programme on Chemical Safety (IPCS) under auspices of World Health Organisation and the United Nations Environment Programme. Extracts of soil samples were prepared by suspending soil in distilled water in ratio of 1:2 (w/v) for about 12 hours at room temperature. Different concentrations of extract $(10 \%$, $25 \%, 50 \%, 75 \%$ and $100 \%$ ) were used for treatment of roots. The extracts were found to be significantly genotoxic at higher concentrations. The genotoxic potential of the soil samples was correlated with content of heavy metals like: chromium, cobalt, copper, manganese, mercury, nickel and zinc. A significant increase in anaphase aberration frequency was observed in roots of Allium cepa with an increase in concentration of heavy metals of soil extracts. Other physicochemical parameters like: $\mathrm{pH}$, alkalinity, water holding capacity, bulk density and moisture content were also studied.

\section{PO:06:80}

\section{Karyotype analysis and physical mapping of 17S and 5S rDNA sites in Anemarrhena asphodeloides Bunge}

S. Kim ${ }^{1}$, W. Lee ${ }^{1}$ and J. Bang ${ }^{1}$

${ }^{1}$ Department of Biology, Chungnam National

University, Daejeon 305-764, Korea

Cytogenetic analysis of a medicinal plant Anemarrhena asphodeloides Bunge belonging to
Haemodoraceae was carried out using Feulgen staining and fluorescence in situ hybridization (FISH). The somatic metaphase chromosome number was $2 \mathrm{n}=2 \mathrm{x}=22$ and the size of chromosomes ranged from 1.27 to 3.80 . Three pairs of chromosomes were relatively long in total length and the others were short. Chromosome complements comprise eight pairs of metacentic (chromosome 2, 3, 6, 7, 8, 9, 10, and 11), two pairs of submetacentric (chromosome 4 and 5), and one pair of subtelocentric (chromosome 1). Using FISH, the $17 \mathrm{~S}$ and $5 \mathrm{~S}$ genes were physically mapped on the metaphase chromosomes. Two pairs of $17 \mathrm{~S}$ signal were detected on the terminal regions of the short arms of homology 1 and 3 . One pair of $5 \mathrm{~S}$ signal was detected on the short arm of chromosome 3 . This $5 \mathrm{~S}$ site seemed to be the same locus as one of the $17 \mathrm{~S}$ rDNA.

Acknowledgement: This research was supported by a grant (Code \#: PF001201-03: Pl J. W. Bang) from Plant Diversity Research Center of 21st Century Frontier Research Program funded by Ministry of Science and Technology of Korean government.

\section{PO:06:81}

\section{The distribiution of repetitive DNA sequences in Chenopodium species}

\section{B. Kolano ${ }^{1}$, B. Gardunia ${ }^{2}$, E. Jellen ${ }^{2}$, M.} Stevens $^{2}$ and J. Maluszynska ${ }^{1}$

${ }^{1}$ Department of Plant Anatomy and Cytology, University of Silesia, Katowice, Poland;

${ }^{2}$ Department of Plant and Animal Sciences,

Brigham Young University, Provo, Utah, USA

Domesticated Chenopodium species have recently attracted interest as seed and fodder crops due to their nutrition value and wide adaptability to different ecological niches. Despite this increased interest the knowledge of their nuclear genome organisation is still limited and need more detailed studies.

Genomic organization of three kinds of repetitive sequences: satellite, retroelement-like and transposone-like were characterised in a $C$. quinoa. Fluorescent in situ hybridization revealed that most of the mobile element-like sequences were localized mainly in pericentromeric region. 
Interstitial or terminal localization occurred more rarely. Satellite sequences were observed as a week but discrete signals dispersed throughout whole chromosome arms.

To compare $C$. quinoa genome organization with related species, hybridization experiments were conducted with the same probes on nuclear genome of $C$. berlandieri (domesticated and wild subspiecies) and C. album. The distribution of the sequences analysed by Southern hybridization revealed that most of the DNA clones were present in $C$. berlandieri genome but not in $C$. album what may suggest that $C$. quinoa is closer related with $C$. berlandieri than with $C$. album. Some differences in genome organization between cultivated and wild $C$. berlandieri subspecies were also shown. FISH to $C$. berlandieri chromosomes exhibited very similar hybridization pattern to this observed in $C$. quinua but number and strength of signals were reduced.

The research was supported by the Polish State Committee for Scientific Research, grant No. 3 P04C 006 24, the Ezra T. Benson Institute, and the McKnight Foundation.

\section{PO:06:82}

\section{Cytogenetic analysis of eight species in Umbelliferae}

\section{Koo ${ }^{1}$, Y. Lee ${ }^{1}$ and J. Bang ${ }^{1}$}

${ }^{1}$ Department of Biology, Chungnam National University, Daejeon 305-764, Korea

Chromosome morphology can be used to clarify the relationships between species. Karyotypes and physical mapping of rDNAs as chromosome marker were investigated from eight medicinal plant species in Umbelliferae. Karyotype formulas are as follows: Angelica acutiloba: $\mathrm{K}(2 \mathrm{n})=22=7 \mathrm{~m}+1 \mathrm{sm}+3 \mathrm{st} ;$ A. sinensis: $\mathrm{K}(2 \mathrm{n})=$ $22=6 \mathrm{~m}+3 \mathrm{sm}+2 \mathrm{st} ; \quad$ A. gigas: $\quad \mathrm{K}(2 \mathrm{n})=22=$ $5 \mathrm{~m}+6 \mathrm{sm} ; \quad$ Bupleurum falcatum: $\mathrm{K}(2 \mathrm{n})=26=$ $12 \mathrm{~m}+1 \mathrm{sm} ;$ B. longeradiatum: $\mathrm{K}(2 \mathrm{n})=12=3 \mathrm{~m}+$ $3 \mathrm{sm}$; Cnidium officinale: $\mathrm{K}(2 \mathrm{n})=3 \mathrm{x}=33=$ $5 \mathrm{~m}+4 \mathrm{sm}+2 \mathrm{st} ;$ Ostericum koranum: $\mathrm{K}(2 \mathrm{n})=22=$ $4 \mathrm{~m}+4 \mathrm{sm}+3 \mathrm{st}$ : O. karanum cv. Suwon: $\mathrm{K}(2 \mathrm{n})=$ $3 \mathrm{x}=33=6 \mathrm{~m}+3 \mathrm{sm}+2 \mathrm{st}$. Chromosomal localization of $5 \mathrm{~S}$ and $45 \mathrm{~S}$ rDNAs using multi-color fluorescence in situ hybridization (McFISH) technique were carried out. In In FISH experiment, one pair of 5S rDNA signals was detected on the pericentromeric region of chromosome 4 and one pair of $45 \mathrm{~S}$ rDNA signals was detected on the telomeric region of chromosome 2 in B. longeradiatum. In Angelica species, the numbers and size of $45 \mathrm{~S}$ rDNA signals were varied between two species, however each signal of the 5S rDNA was observed in two species, A. gigas and A. acutiloba.

Acknowledgments: This work was supported by a grant from BioGreen 21 Program, Rural Development Administration, Republic of Korea.

\section{PO:06:83}

\section{Chromosome sorting in durum wheat as a tool for wheat genome analysis}

\section{Kubalakova ${ }^{1}$, P. Kovarova ${ }^{1}$, P. Suchankova', J. Bartos', J. Cihalikova', S. Lucretti ${ }^{2}$, N. Watanabe ${ }^{3}$, S. Kianian ${ }^{4}$ and J. Dolezel ${ }^{1}$}

${ }^{1}$ Laboratory of Molecular Cytogenetics and Cytometry, Institute of Experimental Botany, CZ-77200 Olomouc, Czech Republic;

${ }^{2}$ ENEA, U.T.S. Biotecnologie per la Protezione della Salute e degli Ecosistemi, 00060 S.M. di Galeria (Roma), Italy; ${ }^{3}$ Faculty of Agriculture, Gifu University, Gifu 501-1193, Japan;

${ }^{4}$ Department of Plant Sciences,

North Dakota State University, Fargo, ND 58105, USA

Molecular analysis of the wheat genome has been hampered by its large size and polyploid nature. Previously, we demonstrated that flow cytometry facilitated this task in hexaploid wheat by purifying chromosomes and chromosome arms. This study evaluates the potential of flow cytometry for chromosome sorting in durum wheat (Triticum durum, $2 \mathrm{n}=4 \mathrm{x}=28$ ). Histograms of fluorescence intensity (flow karyotypes) obtained after the analysis of DAPI-stained chromosomes consisted of three peaks. Of these, one represented chromosome $3 \mathrm{~B}$, a small peak corresponded to chromosomes $1 \mathrm{~A}$ and $6 \mathrm{~A}$, and a large peak represented the remaining eleven chromosomes. Chromosomes sorted onto microscope slides were 
identified after fluorescence in situ hybridisation (FISH) with probes for GAA microsatellite, pSc119.2 and Afa repeats. Genomic distribution of these sequences was determined for the first time in durum wheat and it was found similar to that of A- and B-genome chromosomes of hexaploid wheat. FISH with a probe for GAA microsatellite permitted unambiguous identification of all chromosomes within the karyotype. Flow karyotyping in double ditelosomic lines of durum wheat revealed that the lines could be used to sort any arm of the hexaploid wheat Aand B-genome chromosomes. Compared to hexaploid wheat, flow karyotype of durum wheat is less complex. This results in better discrimination of telosomes and high purities in sorted fractions, approaching 100\%. This work has been supported by the Ministry of Education, Youth and Sports of the Czech Republic (grants ME527 and ME528) and the Ministry of Agriculture of the Czech Republic (grant QC1336).

\section{PO:06:84}

\section{Molecular cytological diversity was narrow among japonica varieties in cultivated rice Oryza sativa}

\section{S. Nakayama ${ }^{1}$}

\author{
${ }^{1}$ National Institute of Agrobiological Sciences, \\ Tsukuba, Japan
}

In cultivated rice Oryza sativa, broad intraspecies diversity has been demonstrated by physiological and molecular biological studies, but a few papers have reported on molecular cytological diversity. To reveal the molecular cytological diversity in $O$. sativa, a tandem repetitive sequence $\mathrm{Os} 48$ ( $\mathrm{Wu}$ and $\mathrm{Wu}$ 1987) was visualized by fluorescence in situ hybridization (FISH) with tyramide signal amplification in various rice varieties, including temperate and tropical varieties of japonica and boro and aman varieties of indica. Diversity was reflected by differences in the number of FISH signals. The number of loci detected was almost the same among japonica varieties, but differed significantly among indica varieties. The difference in Os48 loci reflected a difference in chromosomal structure among the species. On the other hand, repetitive sequences of $45 \mathrm{~S}$ rDNA were also mapped to distal region(s) of chromosome(s) in O. sativa. Two-colored FISH of Os48 and rDNA was also carried out to determine the correlation between their loci and the difference in chromosomal structure among japonica and indica varieties. These results indicated first, that intra-species cytological diversity has accumulated in japonica and in indica rice and second, that the molecular cytological diversity was narrow among japonica varieties, but broad among indica varieties.

\section{PO:06:85}

\section{Meiotic studies of Brassica napus cultivars}

\author{
Z. Nourmohammadi ${ }^{1}$ and M. Sheidai ${ }^{1}$ \\ ${ }^{1}$ Biology Department, Shahid Beheshti University, \\ Tehran, Iran
}

Meiotic study was performed in 22 Brassica napus cultivars considering chiasma frequency and distribution as well as chromosome pairing. All cultivars possessed $n=19$ chromosome number $(4 \mathrm{x})$ and showed a deviant course of prophase-I meiosis with synezetic knot and post pachytene diffuse stage. Chromosome stickiness occurred in most of the cultivars from early prophase to late telophase-II leading to the formation of laggard chromosomes and micronuclei. The cultivars studied differed significantly in chiasma frequency and distribution as well as bivalent formation indicating their genomic differences. Cluster analysis and ordination based on principal components analysis grouped those cultivars showing meiotic similarities. Some of the cultivars showed the occurrence of unreduced meiocytes and pollen grains.

\section{PO:06:86}

Cytological analyses in a fertile hybrid between Vitis vinifera cv. Italia and $V$. rotundifolia cv. Regale 


\section{N. Pierozzi ${ }^{1}$, C. Pinto Maglio ${ }^{1}$ and C. Pommer ${ }^{2}$}

${ }^{1}$ CPD Recursos Genéticos Vegetais. Instituto Agronimico (IAC). Campinas SP Brasil. CEP:13001-970. PO Box 28.; ${ }^{2}$ CAPTA Frutas (IAC)

Vitis vinifera and $V$. rotundifolia were crossed in an attempt to obtain hybrids with disease resistance present in the latter species. Several F1 seedlings were obtained, but only one survived (IAC 3-10) which was cytologically analyzed. Chromosome counts were performed using acetic orcein; chromosome characterizations were done by C-band, -NOR and CMA3 techniques. Pollen counts and measure were done by Alexander method. Leaf stomata number and measure were obtained by cellulose acetate technique. It was confirmed $2 \mathrm{n}=38$ for $V$. vinifera and $2 \mathrm{n}=40$ for $V$. rotundifolia. The hybrid, however, showed $2 \mathrm{n}=38$ chromosomes, an unexpected number. The ideograms obtained showed little difference among them. The C-band was concentrated around centromere. There are two pairs of chromosome with NOR in each species, after AgNOR and CMA3. Pollen grain viability mean value was $75.72 \%$ and grain size mean value was $19.87 \pm 1.51 \mathrm{~mm}$, resembling those of $V$. vinifera . Leaf stomata number mean values per squared area were $122.93 \pm 1.14$ for $V$. vinifera, $386.77 \pm 2.16$ for $\mathrm{V}$. rotundifolia and $255.73 \pm 2.56$ for the hybrid. Leaf stomata length mean values were $31.63 \pm 2.03 \mathrm{~mm}$ for $V$. vinifera and $23.12 \pm 1.80 \mathrm{~mm}$ for $V$. rotundifolia and a mix of both stomata length in the hybrid. IAC 3-10 presents a breakthrough in table grape culture in Brazil due to its potential use directly in super humid regions, if proved resistant, or as an elite parent for the breeding program continuity, or as rootstock for elite grape cultivars.

Financial support: FAPESP.

PO:06:87

\section{Plant autonomous chromosome transfer}

G. Rudgers ${ }^{1}$, J. Mach ${ }^{1}$, J. Jin ${ }^{1}$, H. Zieler ${ }^{1}$ and D. Preuss ${ }^{2}$
${ }^{1}$ Chromatin, Inc, USA $;{ }^{2}$ The University of Chicago, USA

Integrative plant transformation has accelerated the introduction of novel traits into plants and enabled the elucidation of gene function. But, traditional transformation methods, including Agrobacterium mediated transformation and mechanical bombardment, result in imprecise gene integration into the plant host genome with uncertainty concerning transgene integrity, location, or copy number. The result is often unpredictable transgene expression, disruption of the host plant genome with uncertainty concerning transgene integrity, location, or copy number that may only be eliminated after multiple generations of event selection and breeding. To overcome these obstacles we have developed an autonomously replicating plant mini-chromosome that allows for more predictable, reliable, and efficient introduction of genes into crop species without gene integration into the host genome. Species-specific genomic DNA conferring centromere function were combined with a multigene cassette to form autonomous plant minichromosomes which were delivered into cultured plant cells. Whole plants were regenerated after expansion of cell cultures and subsequently demonstrated to contain autonomous minichromosomes with consistent gene expression in differentiated tissues. These results demonstrate that autonomous plant mini-chromosomes can be constructed from isolated plant genomic DNA to produce well defined, heritable and stable linkage groups supporting predictable gene expression in plants without integration into the host genome.

\section{PO:06:88}

\section{Chromosome and genome size} evolution in holoparasitic Orobanche (Orobanchaceae) and related genera

\section{H. Schneeweiss ${ }^{1}$, J. Greilhuber ${ }^{2}$ and G. Schneeweiss ${ }^{3}$}

${ }^{1}$ Department of Higher Plant Systematics and Evolution, Institute of Botany, University of Vienna, Rennweg 14, A-1030 Vienna, Austria; ${ }^{2}$ Department of Systematic Karyology 
and Embryology of Higher Plants, Institute of Botany, University of Vienna, Rennweg 14, A-1030 Vienna, Austria; ${ }^{3}$ Department of Plant Biogeography, Institute of Botany, University of Vienna, Rennweg 14, A-1030 Vienna, Austria

Distribution of basic chromosome numbers and karyotypes in Orobanche and related genera supports three lineages inferred from molecular phylogenetic analyses. Orobanche sect. Orobanche plus Diphelypaea (Orobanche-group) have $\mathrm{x}=19$ and their chromosomes are small to mediumsized mostly meta- to submetacentrics. Orobanche sects. Gymnocaulis, Myzorrhiza and Trionychon (Phelipanche-group) have $\mathrm{x}=12$ and small or medium-sized submeta- to acrocentric chromosomes. Cistanche has $\mathrm{x}=20$ with large meta- to submetacentric chromosomes. Genome size estimates, newly obtained for 44 taxa (more than 70 accessions) using Feulgen densitometry, agree with molecular phylogenetic hypotheses, e.g., the phylogenetically distinct $O$. anatolica has much larger chromosomes and thus higher DNA content than other species of sect. Orobanche.

Polyploidy is found in several lineages. In most cases, polyploids have significantly lower DNA content than related diploid species. For instance, genome size of tetraploid species of sect. Myzorrhiza is $2.16-2.53 \mathrm{pg}$ ( $2 \mathrm{C}$; corrected for ploidy level) compared to $6.76-10.84 \mathrm{pg}$ in the diploid species of sect. Trionychon. The mostly tetraploid Orobanche gracilis and relatives have the smallest genomes of all species investigated (1.66-2.45 pg). Genome size in the tetraploid cytotype of $O$. transcaucasica is nearly twice as high as in the diploid cytotype, suggesting rather recent polyploidisation. Polyploid Orobanche exhibit a clear trend towards sequence elimination. Another evolutionarily important trend is that species attacking short-lived hosts, among them economically important weeds, tend to have lower DNA contents.

\section{PO:06:89}

\section{Cytogenetic features of Iranian pomegranate (Punica granatum L.) cultivars}

\section{Sheidai ${ }^{1}$}

${ }^{1}$ Biology Department, Shahid Beheshti University, Evin, Tehran, Iran

Cytogenetic studies were performed on about 40 Iranian pomegranate (Punica granatum L.) cultivars considering the polyploidy level, chiasma frequency and distribution, chromosome association and segregation, occurrence and effects of B-chromosomes and cytogenetical mechanisms of unreduced $(2 \mathrm{n})$ gametes. All cultivars possessed $\mathrm{n}=8=2 \mathrm{x}$ chromosome number mainly formed bivalents in metaphase but in a few of them quadrivalents were formed due to the occurrence of translocation mainly between the largest chromosome pair of the genome and one of the small chromosome pairs. The cultivars studied differed significantly in their cytogenetical characters indicating their genomic difference. B-chromosome (0-6) occurred in some of the cultivars which were much smaller than the A-chromosome and did not pair with them. The effects of Bs on chiasma frequency and distribution varied in different cultivars and was related to the genotypic background of the cultivar. In some of the cultivars unreduced (potential 2n) meiocytes and pollen grains were formed due to cytogenetical abnormalities such as multipolar cell formation and cytomixis. Details of such cytogenetic phenomena will be discussed.

\section{PO:06:90}

\section{Effect and exploitation of rye genome in wheat improvement}

\section{Sidhu ${ }^{1}$ and C. Satija ${ }^{2}$}

${ }^{1}$ Department of Botany, Panjab University, Chandigarh, India; ${ }^{2}$ Department of Genetics and Biotechnology, PAU, Ludhiana, India

Rye genome is known to carry genes for resistance to diseases and adaptability to abiotic stresses. Wheat-rye disomic/ditelosomic addition lines have been used to incorporate specific rye chromosomes/part of chromosome to wheat. The hybrid progenies of elite wheat genotypes with wheat-rye disomic/ditelosomic addition lines in Chinese Spring background revealed differential effect of rye chromosome on morphology, disease resistance and productive potential. 
Crossability was fairly high among addition lines with all wheat genotypes but the variation was genotypic dependent. Truthfulness of hybrid progenies was established through the presence of awns as well as chromosome number $(2 n=43)$. Hybrid and their self-progenies with rye chromosome 4 and 5 particularly short arm impart resistance to Karnal bunt caused by Tilletia indica. The rye chromosome 4 and 7 as well as long arm of chromosome 2 and 4 has genes for increased spike length. Chromosome 5 and short arm of chromosome 3 have alleles for increasing number of spikelets. Grain weight was influenced by chromosome 6, 7 and short arm of 4 and addition of these chromosomes results in high grain weight. To exploit desirable parts of rye genome, the progenies having rye chromosomes need to be back crossed with wheat genotypes and selecting for 42-chromosome constitution. The progenies with better performance for quality and resistance can be selected which will increase productivity with diversity.

\section{PO:06:91}

\section{Mre11-deficient Arabidopsis exhibit aberrant meiosis and chromosomal instability in somatic cells}

\section{J. Siroky', J. Puizina ${ }^{2}$, P. Mokros ${ }^{1}$, D. Schweizer ${ }^{2}$ and K. Riha ${ }^{2}$}

${ }^{1}$ Institute of Biophysics, Czech Academy of Sciences, Brno, Czech Republic; ${ }^{2}$ Gregor Mendel Institute of Molecular Plant Biology, Austrian Academy of Sciences, Vienna, Austria

The Mre11 complex, initially described in budding yeast, is composed of Mre11, Rad50 and Nbs1 proteins, and acts in manifold cellular functions such as DNA checkpoint mechanisms, double-strand break repair, regular meiotic recombination, and telomere maintenance. We report the outcome of Mre11 deficiency for the stability of mitotic and meiotic chromosomes in Arabidopsis. Plants homozygous for the mre11 mutation exhibit vegetative growth defects and are infertile. Analysis of mitotic chromosomes in mutant plants revealed a number of anaphase bridges during divisions and abundant chromo- some acentric fragments. Multicolour FISH with $\mathrm{BAC}$ probes specific for individual chromosome arms showed that the anaphase bridges are often formed by fusion of homologous chromosome arms. The frequency of chromosome fusions was not reduced in mre11 ku70 double mutants, sug gesting that plants possess robust DNA endjoining activities independent of the $\mathrm{Ku} 70 / 80$ and Mre11 complexes. We also detected erroneous synapsis in the pachytene stage and severe chromosome fragmentation in the initial stages of male meiosis. This fragmentation was substantially suppressed in mre11 spo11-1 double mutants, indicating that Mre11 is required for repair, but not for the induction of Spol1dependent meiotic DNA breaks in Arabidopsis. The presented data play up differences in the function of Mre11 in the initial stages of meiotic recombination between yeast and Arabidopsis.

\section{PO:06:92}

\section{Progress in flow cytogenetics of barley}

P. Suchankova ${ }^{1}$, M. Kubalakova ${ }^{1}$, P. Kovarova ${ }^{1}$, J. Bartos' ${ }^{1}$, J. Cihalikova ${ }^{1}$, T. Endo ${ }^{2}$ and J. Dolezel ${ }^{1}$

${ }^{1}$ Laboratory of Molecular Cytogenetics and Cytometry, Institute of Experimental Botany, Sokolovska 6, CZ-77200 Olomouc, Czech Republic; ${ }^{2}$ Laboratory of Plant Genetics, Graduate School of Agriculture, Kyoto University, Kyoto 606-8502, Japan

Flow cytogenetics is an attractive tool that simplifies the analysis of complex plant genomes. Flow-sorted chromosomes have been used for the isolation of molecular markers from targeted genome regions, for physical mapping using PCR and FISH, and for the construction of chromosome-specific DNA libraries. Until now, the application of flow cytogenetics in barley has been hampered by the inability to sort individual chromosomes, except the smallest chromosome $1 \mathrm{H}$ and some translocation chromosomes with DNA content different from the remaining chromosomes. However, although the sorted fractions of translocation chromosomes have been useful for mapping DNA sequences to subchromosomal regions, their use remains limited. In this work, 
we demonstrate that wheat-barley telosome addition lines may be used to sort any of the fourteen barley chromosome arms. Identity of sorted arms can be verified using chromosome-specific markers; the purity in sorted fractions can be assessed by FISH. Furthermore, we will be able to sort subarm chromosomal segments that are generated in the wheat-barley chromosome addition lines by the gametocidal system. These advances make barley flow cytogenetics an attractive tool that may greatly facilitate its genome mapping. A possibility to purify large quantities of individual chromosome arms opens avenues for targeted isolation of low-copy sequences, preparation of specific probes for screening EST arrays, HAPPY mapping, and construction of chromosome arm-specific BAC libraries.

This work has been supported by the Ministry of Education, Youth and Sports of the Czech Republic (grants ME527 and ME528) and the Ministry of Agriculture of the Czech Republic (grant QC1336).

\section{PO:06:93}

\section{Characterization of a telomere end binding protein in Arabidopsis thaliana}

\section{A. Tani ${ }^{1}$ and M. Murata ${ }^{2}$ \\ ${ }^{1}$ Graduate School of Natural Science and Technology, Okayama University, Okayama 700-8530, Japan; ${ }^{2}$ Research Institute for Bioresources, Okayama University, Kurashiki 710-0046, Japan}

Pot1 (protection of telomere) is a G-rich singlestranded telomeric DNA binding protein, identified first in Schizosaccharomyces pombe, and shown to play an important role in stabilizing chromosomes. Pot1-like proteins or their encoding genes have been identified from yeasts to mammals. Based on the $\mathrm{N}$-terminal amino acid sequences of fission yeast and human Pot1, two Pot1-like proteins (AtPot1 and AtPot2) were identified in Arabidopsis thaliana, but neither of them was characterized. In this study, we attempted to amplify full-length cDNAs corresponding to AtPot1 by RT-PCR, which has higher similarity to human Pot1 (hPot1). As a result, the RTPCR products contained three different DNA sequences. This suggests that AtPot1 is transcribed in three splicing variants, and has additional unpredicted exons. Alternative splicing was also known to occur in hPot1, and only one splicing variant had tissue specificity among five splicing variants. Therefore, tissue specificity was investigated also in AtPot1, but all three splicing variants were detected in all tissues examined, including buds, flowers, leaves, roots, steams, siliques and cultured cells. Northern blot hybridization indicated that AtPot1 expresses more in meristematic tissues than in vegetative tissues. Nuclear localization of AtPot1 was confirmed by GFP-fusion experiments, but the telomere localization was not decided yet.

\section{PO:06:94}

\section{Cytogenetic effect of nitrosomethylurea and its modification by hyperbaric oxigenation}

\section{A. Usatov ${ }^{1}$, E. Mashkina ${ }^{1}$ and E. Guskov ${ }^{1}$}

${ }^{1}$ Research Institute of Biology, Rostov State University, Rostov-on-Don, 344090 Russia

Cytogenetic effect of nitrosomethylurea (NMU) depends on the concentration of mutagen. NMU-treatment of sunflower seedlings $(0.015 \%$ NMU) reduces proliferation activity of root meristeme \&\#1089; ells, leaving the level of chromosome aberrations unchanged, while $0.03 \%$ concentration of NMU increases the level of chromosome aberration as early as 12 hours after the treatment was over. Mutagenesis is a complicated process depending on many internal and external factors, and our research was aimed on investigation of the possibilities of modification of NMU-induced cytogenetic effect by HBO.

Used in non-inducing chromosome aberrations mode, HBO enhances cytogenetic effect of NMU. The joint action of NMU and HBO significantly inhibits the proliferation activity of cells. When HBO was used before NMU-treatment $(0,015 \%)$, the level of chromosome aberrations increased reliable as compared not only to control, but to NMU-treated group as well. Furthermore, HBO pretreatment prolongates the cytogenetic effect of NMU $(0.03 \%)$, while HBO post-treatment does 
not increase the level of NMU-induced chromosome aberrations.

We suggests that there is specific mechanism for interaction between HBO and NMU. Apparently, modification of NMU-effect is associated to formation of mutagenic products of NMU metabolism and their interaction with active forms of oxygen. It is possible that during cell metabolism of nitrogen-contained compounds nitrogen oxide is formed, being capable to interact with superoxide to produce high-toxic peroxynitrite.

We suppose that enhancing of NMU-mutagenesis by HBO may be associated with the formation of peroxynitrite; the latter can to induce DNA damage directly, to activate free-radical processes and lipid-peroxidation reactions.

\section{Prokaryote Chromosomes Symposium}

\section{L91}

\section{Pole to pole oscillation of an ATPase is required for $\mathbf{F}$ plasmid segregation in E.coli}

\section{A. Wright ${ }^{1}$ and G. Leung ${ }^{1}$}

${ }^{1}$ Tufts Medical School, Boston, MA 02111, USA

Segregation of the low copy plasmid, F, in E.coli, is dependent on plasmid specified partition functions which include a centromere like site (SopC ) and two trans-acting genes, one encoding an ATPase (SopA) the other encoding a protein (SopB) which binds to the centromere forming a large nucleoprotein complex. Interactions between the ATP-bound form of SopA and the SopB-centromere complex result in stimulation of its ATPase activity, resulting in conversion of SopA-ATP to SopA-ADP and under some conditions, release of SopB from the centromere. We have examined the intracellular distribution of the two partition proteins, SopA and SopB and their relationship with plasmid DNA by fluorescence microscopy, using GFP and its derivatives to ãtagä each component. Prior to segregation $F$ plasmid DNA is localized at or near the cell center in complex with a large number of
SopB molecules, seen as fluorescent foci. SopA, in contrast, behaves in a dynamic manner, oscillating from pole to pole in a spiral track, creating a concentration gradient, highest at the cell poles and lowest at the cell center. SopA oscillation is absolutely dependent on the ATPase activity of SopA. Segregation of plasmid DNA, is preceded by dissociation of plasmid DNA from SopB followed by a redistribution of SopB protein complexes to the cell quarter positions where they act as receptors for sister plasmid molecules. We will discuss the role of the SopA gradient in plasmid localization and partition.

\section{L92}

\section{Visualising chromosome segregation in vivo and in vitro}

\section{Sherratt ${ }^{1}, X$. Wang ${ }^{1}$, C. Possoz ${ }^{1}$ and S. Filipe ${ }^{1}$}

${ }^{1}$ Biochemistry Dept, University of Oxford, UK

We analyse the spatial and temporal organisation of chromosome origins, termini, replication proteins, recombination proteins, segregation proteins and cell division apparatus in growing E.coli cells. These data are then related to a model for how DNA processing and cell division are coordinated. Finally we demonstrate chromosome segregation in an in vitro single molecule experiment.

\section{L93}

\section{Dual architectural roles of the nucleoid-associated protein $\mathrm{HU}$ from $E$. coli}

\section{J. Van Noort ${ }^{1}$, S. Verbrugge ${ }^{1}$, N. Goosen ${ }^{2}$, C. Dekker ${ }^{1}$ and R. Dame ${ }^{3}$}

${ }^{1}$ Molecular Biophysics, Department of Nanoscience, TU Delft, The Netherlands; ${ }^{2} \mathrm{Lab}$ of Molecular Genetics, Universiteit Leiden, The Netherlands; ${ }^{3}$ Physics of Complex Systems, Vrije Universiteit, Amsterdam, The Netherlands

Bacterial chromatin is thought to be organized and compacted at least in part by interaction 
with nucleoid-associated proteins. One of these proteins, HU, has long been thought to act in a fashion similar to eukaryotic histones i.e. by wrapping of DNA around a protein core (1). Using a combination of single molecule techniques we demonstrate that HU does not employ this type of mechanism to compact DNA. Scanning force microscopy studies show individual HU molecules that induce sharp bends. Under similar conditions magnetic tweezers studies reveal compaction of DNA up to about $50 \%$ of its original length. At high concentrations of $\mathrm{HU}$ both techniques indicate an increased rigidity of the DNA helix. SFM height images of such rigid complexes show that this is due to the formation of an HU filament around DNA with a superhelical repeat of $\sim 16 \mathrm{~nm}$. Taken together these data provide firm evidence that DNA compaction by HU stems from the (dynamic) induction of local bends into DNA rather than from DNA wrapping (2).

\section{References}

(1) Dame, R.T. and Goosen, N. (2002). FEBS Lett 529, $151-156$

(2) van Noort. J., Verbrugge, S., Goosen, N., Dekker, C. and Dame, R.T. Proc Natl Acad Sci USA. In press.

\section{L94}

\section{Coordination of chromosome} segregation and cell division by a nucleoid occlusion protein in Bacillus subtilis

\section{J. Errington ${ }^{1}$ and L. $\mathrm{Wu}^{1}$ \\ ${ }^{1}$ Sir William Dunn School of Pathology, University of Oxford, UK}

Efficient chromosome segregation in bacteria is probably achieved by several interacting and partially redundant processes. Segregation is coordinated with cell division such that, normally, division occurs in the space between the separating sister chromosomes. Selection of the correct mid cell site for division in rod-shaped bacteria is thought to be governed by the combined action of two negative regulators comprising the well characterized Min system, which blocks division in the vicinity of the cell poles, and a hypothetical nucleoid occlusion system that blocks division in the vicinity of the nucleoid. The nucleoid occlusion system would operate to help direct the division machinery to the space between the segregating nucleoids. We have identified the key player in nucleoid occlusion in B. subtilis, as a novel inhibitor of division that is also a nonspecific DNA-binding protein. The nucleoid occlusion protein, Noc, is closely related to a key chromosome segregation protein, Spo0J. Under various conditions in which the cell cycle is perturbed Noc prevents the division machinery (FtsZ ring) from assembling in the vicinity of the nucleoid. Unexpectedly, cells lacking both of the topological inhibitors of division (i.e. Min and Noc) are blocked for division because they establish multiple non-productive FtsZ accumulations. The results help to explain how B. subtilis identifies the mid cell site for division and avoids catastrophic breakage of the chromosome by division through the nucleoid.

\section{L95}

\section{Rings and moving helices: the SMC complex and actin-like MreB protein are part of the prokaryotic chromosome segregation machinery}

\author{
J. Mascarenhas' ${ }^{1}$, A. Volkov ${ }^{1}$, H. Defeu Soufo ${ }^{1}$ \\ and P. Graumann ${ }^{1}$ \\ ${ }^{1}$ Biochemie, Fachbereich Chemie, \\ Hans-Meerwein-Straße, Philipps-Universität \\ Marburg, 35043 Marburg, Germany
}

Bacteria contain an active intracellular segregation machinery that separates sister chromosomes towards opposite cell poles during ongoing replication. An important component of this machinery is the SMC complex, which is required for complete separation of chromosomes (but not for initial separation of origin regions) and for proper chromosome organization in Bacillus subtilis. SMC forms a ternary complex with two proteins, ScpA and ScpB, that are conserved in prokaryotes, and localizes in two distinct subcellular centers within both cell halves. From there, the 
complex condenses and organizes DNA that is duplicated through the centrally located DNA replication machinery. SMC binds to DNA as an unusual ring like structure, condensing DNA by embracing DNA loops. DNA binding is mediated through ATP binding at the head domains, which leads to dimerization of the heads and thus to ring closure.

We have recently found that the origin regions fail to separate in the absence of $B$. subtilis actinlike proteins MreB and Mbl. Both proteins form dynamic helical filaments that move with a speed of $0.1 \mu \mathrm{m} / \mathrm{s}$ underneath the cell membrane, suggesting that they could be the motor that pushes DNA towards opposite poles. MreB and $\mathrm{Mbl}$ also affect cell shape, same as the $\mathrm{MreC}$ and $\mathrm{MreD}$ proteins whose genes are downstream of mreB. Both $\mathrm{MreC}$ and $\mathrm{MreD}$ are membrane proteins, which might provide the link between chromosome segregation and formation of rod shaped cells.

\section{PO:08:01}

\section{The mechanism of DNA compaction by} the nucleoid-associated protein $\mathrm{H}-\mathrm{NS}$ from $E$. coli

\author{
R. Dame ${ }^{1}$, M. Noom ${ }^{1}$, N. Goosen ${ }^{2}$ and \\ G. Wuite ${ }^{1}$
}

${ }^{1}$ Physics of Complex Systems, Vrije Universiteit, Amsterdam, The Netherlands; ${ }^{2}$ Lab of Molecular Genetics, Universiteit Leiden, Leiden,

The Netherlands

Bacterial chromatin is thought to be organized and compacted at least in part by interaction with nucleoid-associated proteins. Recently we have shown that one of these proteins, H-NS, compacts DNA by the formation of bridges between adjacent DNA tracts [1]. This property is exploited not only for providing a means of compaction, but is also essential to the regulatory role of H-NS in transcription. Bridging of DNA provides an explanation for the recognition of curved DNA and for the trapping of RNAP in the open initiation complex at the $\operatorname{rrnB} \mathrm{P} 1$ [2].

In order to further characterize the mechanism underlying DNA compaction by $\mathrm{H}-\mathrm{NS}$, we have performed DNA stretching experiments with optical tweezers. These experiments suggest that the
DNA is organized in independent $\mathrm{H}-\mathrm{NS}$ bridged DNA loops, which can be disrupted by applying an increasing force.

\section{References}

[1] Dame, R.T.; Wyman, C.; Goosen, N. H-NS mediated compaction of DNA visualised by atomic force microscopy. (2000) Nucleic Acids Res 28:3504-10.

[2] Dame, R.T.; Wyman, C.; Wurm, R.; Wagner, R.; Goosen, N. (2002) Structural basis for H-NS mediated trapping of RNA polymerase in the open initiation complex at $\operatorname{rrnB} \mathrm{P} 1$.

\section{PO:08:02}

\section{Spatial and temporal dynamics of chromosome segregation in Vibrio cholerae}

\section{Fogel ${ }^{1}$ and M. Waldor ${ }^{1}$}

${ }^{1}$ Departments of Genetics and Molecular Microbiology, Sackler School of Biomedical Sciences, Tufts University School of Medicine and Howard Hughes Medical Institute, Boston MA 02111, USA

Bacterial chromosome segregation has been studied in detail only in species with single chromosomes. Little is known about chromosome segregation in the growing group of bacteria with multipartite genomes. The human diarrheal pathogen Vibrio cholerae is a gramnegative, gamma-proteobacteria with two circular chromosomes. Using still and time-lapse fluorescence video-microscopy we have visualized the spatial and temporal dynamics of the $V$. cholerae chromosomes. In all stages of the cell cycle, the two origins are localized to distinct sub-cellular locations. In newborn cells, the origin of chromosome 1 (Ori1) is located near the new pole and the origin of chromosome 2 (Ori2) at the cell center. Oril segregates to the poles early in the cell cycle whereas Ori2 segregates close to the time of division and repositions rapidly to the new cell centers. Staining of the nucleoid with 4',6-diamidino-2-phenylindole (DAPI) demonstrates that both origins are associated with the edge of the nucleoid in new cells and that later in the cell cycle, Oril can be positioned far from 
the nucleoid mass, a finding consistent with models of dynamic positioning of the origin regions. The low frequency of overlap of the origin foci with the DAPI fluorescence suggests the origins are sequestered away from the rest of the nucleoid. The coordinated dynamics observed would require segregation machinery that can discriminate between chromosomes and specify their localization. We are currently investigating the roles of the orthologs of plasmid partitioning proteins present on each chromosome.

\section{Sex Chromosomes Symposium}

\section{L96}

\section{The origin, evolution and future of human sex chromosomes}

\section{J. Marshall-Graves ${ }^{1}$}

${ }^{1}$ Research School of Biological Sciences, The Australian National University, Canberra, ACT, Australia

In mammals including humans, sex is determined by an XY male: XX female system in which a male-dominant gene on the Y (SRY) determines testis, and embryonic testis determines maleness. The $\mathrm{X}$ and $\mathrm{Y}$ chromosomes evolved from an ordinary autosome pair as the $\mathrm{Y}$ chromosome was progressively degraded. This must have happened within the last 300 million years, since birds and reptiles have completely non-homologous sex chromosome systems.

To explore the origin of the human Y chromosome and SRY, we compared $\mathrm{X}$ and $\mathrm{Y}$ chromosomes of the three major mammal groups (eutherians, marsupials and monotremes). This allowed us to subdivide both the human $\mathrm{X}$ and $\mathrm{Y}$ into a ancient region shared by all other mammals, and a recently added region that is on the $\mathrm{X}$ and $\mathrm{Y}$ only in placental mammals. The ancient and added regions are also separate, and are further subdivided, in the chicken.

The human $\mathrm{Y}$ chromosome is much smaller than the $\mathrm{X}$, and contains only 45 unique and active genes, survivors of the ongoing process of Y degradation. Many or most of these genes - even those with important functions in male determination and differentiation such as SRY and RBMY - have partners on the $\mathrm{X}$ from which they obviously evolved. Our comparative studies show that almost all of the original human $\mathrm{Y}$ has been lost, and the $\mathrm{Y}$ was saved from extinction only by its autosomal addition.

How much longer can the human Y hold out against the evolutionary forces that degrade it?

\section{L97}

\section{Features of facultative heterochromatin regulated by XIST expression}

\author{
C. Brown ${ }^{1}$, J. Chow ${ }^{1}$, N. Thorogood ${ }^{1}$, L. Hall ${ }^{2}$ \\ and J. Lawrence ${ }^{2}$ \\ ${ }^{1}$ Department of Medical Genetics, \\ University of British Columbia, Vancouver, BC \\ V6T 1Z3, Canada; ${ }^{2}$ University of Massachusetts \\ Medical Centre, Worcester, Massachusetts 01655, \\ USA
}

Expression of XIST, which encodes a large, heterogeneous, nuclear, non-coding RNA, is essential for $\mathrm{X}$ chromosome inactivation. To determine which features of the heterochromatic inactive $\mathrm{X}$ chromosome are directly related to XIST expression we are studying features generally associated with the inactive $\mathrm{X}$ after induction of XIST expression. Features examined include gene silencing, replication timing, histone modifications, incorporation of macrohistone $\mathrm{H} 2 \mathrm{~A}$, and DNA methylation. We are utilizing two different somatic cell-based model systems for the induction of XIST expression. In the first, human and mouse XIST/Xist expression has been induced by 5azacytidine demethylation of active $\mathrm{X}$ chromosomes in a human/mouse somatic cell hybrid. In the second, human XIST expression is induced from ectopic sites in human somatic cells. Variable localization of XIST in these cells will allow us to address how cell type and integration site influence XIST localization. Consistent with previous reports, we detect no silencing in the mouse/human somatic cell hybrid, even in the presence of Xist localization. However, in human cells features of inac- 
tivation can be observed upon induction of XIST. These systems thus provide a means of evaluating the role of XIST in establishing the features of facultative heterochromatin in somatic cells in the presence and absence of transcriptional silencing.

\section{L98}

\section{Evolution in the light of genome} conservation: Sex chromosomes direct morphological and functional diversification

\author{
H. Hameister ${ }^{1}$, M. Kohn ${ }^{1}$ and \\ H. Kehrer-Sawatzki ${ }^{1}$ \\ ${ }^{1}$ Dept. Human Genetics, University Ulm, \\ D-89070 Germany
}

The Genome Project has confronted us with the surprising fact that the human genome contains less than 25000 genes and further, that the number of genes has remained nearly the same since the appearance of the first vertebrates some 400 million years ago. Nevertheless very different species evolved which proves the functional plasticity inherent to this genome. Genome wide gene function tests reveal the special role of sex chromosomes for the development of new traits. This contrasts with the fact that comparative gene mapping data have shown the $\mathrm{X}$ chromosome to be the most conserved gene arrangement. To study the dominant role or balancing act of sex chromosomes a comparison of birds and mammals seems highly informative. Originating from the same ancestral vertebrate genome the birds have developed $\mathrm{Z}$ and $\mathrm{W}$ sex chromosomes, which are genetically different from the $\mathrm{X}$ and $\mathrm{Y}$ sex chromosomes in mammals. We compare the expression pattern in mouse and chicken of $\mathrm{X}$ chromosomal genes. We have chosen $\mathrm{X}$ chromosomal mental retardation, MRX, genes which are human speciation genes, recruited to telencephalic function only recently. It can be shown that in the chicken these genes do not serve the same telencephalic function. Especially informative are those MRX genes which in mammals are also expressed in the testis. Again in chicken no testis specific function for these genes is evident. In conclusion: Recruitment of the same genes to different functions or evolution of the transcription regulatory systems is an effective mechanism during evolution to higher complexity.

\section{L99}

The chicken $\mathrm{Z}$ chromosome: A turn on or a turn off?

H. McQueen
L. Battle-Morera

${ }^{1}$ ICMB, University of Edinburgh, Scotland;

${ }^{2}$ Oxford Radcliffe Hospitals Genetics

Laboratories, Oxford, UK

Birds undergo genetic sex determination using a ZW sex chromosome system. Although the avian mechanisms of neither sex determination nor dosage compensation are understood, a female specific non-coding RNA (MHM) is expressed soon after fertilisation from the single $\mathrm{Z}$ chromosome and is likely to have a role in one or both processes. We have now discovered a prominent female specific modification to the $\mathrm{Z}$ chromatin which co-incides with the MHM locus. The same histone modification is highly enriched on the Drosophila melanogaster male X chromosome and forms an integral part of the mechanism for dosage compensation in this species. We suggest that these female sex chromosome specific modifications could be associated with the mechanism of sex determination or, alternatively, dosage compensation in birds which might operate in a manner analogous to that seen for the fruitfly.

\section{L100}

\section{Ectopic homologous recombination} events in the human $Y$ chromosome

J. Lange ${ }^{1}$, H. Skaletsky ${ }^{1}$, S. Repping ${ }^{2}$, S. Van daalen ${ }^{2}$, C. Korver ${ }^{2}$, L. Brown ${ }^{1}$, S. Rozen ${ }^{1}$ and D. Page ${ }^{1}$

${ }^{1}$ Howard Hughes Medical Institute, Whitehead Institute and Department of Biology, Massachusetts Institute of Technology, 9 
Cambridge Center, Cambridge, Massachusetts 02142, USA; ${ }^{2}$ Center for Reproductive Medicine, Department of Obstetrics and Gynecology, Academic Medical Center, Amsterdam, The Netherlands

A prominent feature of the human Y chromosome is the abundance of amplicons strewn throughout the long and short arms. These large, nearly identical repeat segments comprise approximately $30 \%$ of the male-specific euchromatic sequence, and are present as both direct and indirect repeats. Nine testis-specific gene families, each with multiple copies in the $\mathrm{Y}$ chromosome, are found entirely within the ampliconic region. It has been proposed that gene conversion has maintained the $>99.9 \%$ similarity of sequence pairs, evidence that productive recombination on the $\mathrm{Y}$ chromosome is not limited to its pseudoautosomal regions. However, these amplicons also provide substrates for nonproductive recombination. Several recurrent deletions in the $\mathrm{Y}$ chromosome associated with spermatogenic failure have been molecularly characterized, and are due to non-allelic homologous recombination between amplicons. Our data reveal a novel class of ectopic recombination events predicted by the ampliconic structure.

\section{L101}

\section{Multi-copy $Y$ genes and their role in sperm development}

P. Burgoyne ${ }^{1}$, A. Toure ${ }^{1}$, S. Mahadevaiah ${ }^{1}$, J. Turner ${ }^{1}$, P. Ellis ${ }^{2}$, R. Furlong ${ }^{2}$, E. Clemente ${ }^{2}$ and N. Affara ${ }^{2}$

${ }^{1}$ MRC National Institute for Medical Research, London, UK; ${ }^{2}$ Cambridge University Department of Pathology, Cambridge, UK

The mammalian Y chromosome has an unusually high proportion of genes present in multiple copies; in man it has been shown that these multi-copy genes are organised in large palindromic repeats. In the mouse, lack of the male-specific region of the $\mathrm{Y}$ long arm (MSYq) leads to severe sperm abnormalities and consequent infertility; less extensive deletions result in mild sperm defects compatible with fertility, although sperm function is impaired in vitro.

We are using microarray analysis of the testis transcriptome of mice with three different MSYq deficiencies to provide an overview of the transcriptional changes that underlie the sperm abnormalities. In addition to the expected down regulation of members of the previously chacterised multi-copy Ssty gene family, two other testis-expressed multi-copy MSYq genes were identified. 'Transgene rescue' strategies using BAC genomic clones are being used in attempts to identify the relationship between the sperm abnormalities and specific Y gene deficiencies. The other major transcriptional change identified was the marked upregulation of a number of $\mathrm{X}$ linked genes, some of which are also present in multiple copies, suggesting that there are regulatory interactions between $\mathrm{X}$ and $\mathrm{Y}$ genes during spermiogenesis. The possible basis of these regulatory interactions will be discussed.

\section{L102}

\section{Partial deletions of the AZFc interval of the human $Y$ chromosome and male infertility}

N. Machev' ${ }^{1}$, N. Saut ${ }^{1}$, P. Terriou ${ }^{2}$, M. Guichaoua ${ }^{3}$, P. Collignon ${ }^{4}$, J. Chiaroni ${ }^{5}$, A. Navarro ${ }^{1}$, N. Levy ${ }^{1}$, M. Mattei ${ }^{1}$ and M. Mitchell ${ }^{1}$

${ }^{1}$ Inserm U.491, Faculté de Médecine, 13385 Marseille, France; ${ }^{2}$ Institut de Médecine de la Reproduction, 13008 Marseille, France; ${ }^{3}$ Laboratoire de Spermiologie, Hopital de la Conception, 13385 Marseille, France; ${ }^{4}$ Service de Génétique, Centre Hospitalier de Toulon, 83056 Toulon, France; ${ }^{5}$ Etablissement Français du Sang Alpes-Mediterranee, 13005 Marseille, France

Microdeletions of the AZFc interval of the $\mathrm{Y}$ chromosome are currently the most common known genetic cause of human male infertility. However most AZFc microdeletion are homogeneous in extent and smaller deletions must now be identified and characterised if we are to learn how the AZFc genes contribute to human fertility. Two partial AZFc deletions (gr/gr and b1/ 
b3) have recently been identified in infertile and fertile populations that remove some copies of all $\mathrm{AZFc}$ genes, and an association study indicates that the resulting reduction in gene dose represents a risk factor for spermatogenic failure. To determine the incidence of different partial AZFc deletions and their effect on fertility, we combined quantitative and qualitative analyses of the AZFc interval at the DAZ and CDY1 loci in 300 infertile men and 399 control men. We detected 34 partial AZFc deletions (32 gr/gr deletions), arising from at least 19 independent deletion events, and find $\mathrm{gr} / \mathrm{gr}$ deletion in $6 \%$ of infertile and $3.5 \%$ of control men $(P>0.05)$. Our data provide evidence for two large AZFc inversion polymorphisms, and for relative hot and cold spots of unequal crossing-over within the blocks of homology that mediate gr/gr deletion. Using SFVs (sequence family variants) we discriminate DAZ1/2, DAZ3/4, CDY1a (proximal) and CDY1b (distal) and define four types of DAZ/ CDY1 gr/gr deletion. Only deletions of CDY1a and DAZ3/4 were not found in our fertile population, and show a weak association with infertility $(\mathrm{P}=0.042)$, suggesting that most, but not all, $\mathrm{gr} / \mathrm{gr}$ deletions are neutral variants.

\section{L103}

\section{The fate of the mammalian $Y$ chromosomes}

\section{Filatov ${ }^{1}$}

${ }^{1}$ School of Biosciences, University of Birmingham, Edgbaston, Birmingham B15 2TT, UK

Although X- and Y-chromosomes are thought to evolve from a homologous pair of autosomes, they are extremely different from each other in many organisms, including mammals. Actively recombining $\mathrm{X}$ chromosomes are gene-rich, while the non-recombining Y-chromosomes have undergone the process of genetic degeneration due to reduced efficacy of adaptive and purifying selection in the non-recombining regions. As a result, Y-linked genes accumulate deleterious mutations, and have a reduced ability to adaptive evolution. What is the further fate of the genetically degenerate mammalian Y-chromo- somes? Will they degenerate further and gradually disappear, leading to $\mathrm{X} 0$ males, or even to extinction of males, resulting in apocalyptic extinction of many species? This scenario is unlikely because the efficacy of population genetic processes causing genetic degeneration depends on the number of functional genes linked together on the non-recombining Y-chromosomes. With only few functional genes left on the modern mammalian Y-chromosomes, these forces may be too weak to cause further genetic degeneration. Here I report the evidence that, adaptive evolution is fairly common in the mammalian Ylinked genes, suggesting that genetic degeneration of the mammalian Y-chromosomes does not actively proceed further.

\section{L104}

\section{Chromosomal radiation of the African pygmy mice, subgenus Nannomys (Rodentia; Muridae): a karyotypic and phylogenetic analysis}

\author{
V. Frederic ${ }^{1}$, C. Josette ${ }^{1}$, C. Pascale ${ }^{2}$ and \\ B. Janice ${ }^{1}$
}

${ }^{1}$ Institut des Sciences de l'Evolution (UMR5554), Génétique \& Environnement, Université

Montpellier II, Montpellier, France; ${ }^{2}$ Institut des Sciences de l'Evolution (UMR5554),

Paléontologie, Paléobiologie et Phylogénie, Université Montpellier II, Montpellier, France

The African pygmy mice are small-sized rodents widespread throughout sub-Saharan Africa belonging to one of the four subgenera of the genus Mus (subgenus Nannomys). Owing to their highly conserved morphology, diagnostic characters are scarce leading to ambiguous discrimination of the currently recognized 19 species. On the contrary, chromosomes have been shown to be useful taxonomic markers, as several studies have uncovered extensive karyotypic evolution within this group involving rearrangements such as centric fusions between autosomes, between sex chromosomes and autosomes, and even deletion of sex chromosomes. An analysis of molecular (cytochrome b) and chromosomal markers was performed on speci- 
mens from 12 countries, to assess the role of chromosomal rearrangements in the evolutionary history of this species complex.

The molecular phylogeny supported the monophyly of this group, and resolved seven major clades, most of them characterized by diagnostic chromosomal rearrangements or karyotypes. One of the clades clustered taxa carrying different sex-autosome fusions, in some cases associated with partial or total deletions of sex chromosomes. As these fusions are expected to be highly deleterious, they are rarely observed in mammals and when present, are considered as efficient reproductive isolating mechanisms. The clustering of these taxa into one clade, if confirmed, would support the occurrence of shared genomic traits allowing the formation and/or fixation of such rearrangements in taxa within this clade. The diversity of this type of rearrangement within Nannomys offers the unique opportunity to investigate the molecular basis of sexautosome fusions and the evolutionary consequences on speciation rates and sex determination mechanisms.

\section{L105}

\section{Molecular differentiation of $\mathrm{W}$ chromosomes in Lepidoptera}

\section{F. Marec ${ }^{1}$, M. Vitkova ${ }^{2}$, I. Fukova ${ }^{2}$,} A. Yoshido ${ }^{3}$, K. Sahara ${ }^{3}$ and W. Traut ${ }^{4}$

${ }^{1}$ Institute of Entomology ASCR, CZ-370 05 Ceske Budejovice, Czech Republic; ${ }^{2}$ Faculty of Biological Sciences, University of South Bohemia, CZ-370 05 Ceske Budejovice, Czech Republic; ${ }^{3}$ Graduate School of Agriculture, Hokkaido University, Sapporo 060-8589, Japan; ${ }^{4}$ Institute of Biology, Medical University, D-23538 Lubeck, Germany

In Lepidoptera (moths and butterflies), the majority of species with identified sex chromosomes possess a WZ/ZZ (female/male) system or its numerical variations. The $\mathrm{W}$ and $\mathrm{Z}$ chromosomes show various degrees of structural differentiation from undetectable to obviously non-homologous. In most species, $\mathrm{W}$ chromosomes are largely composed of heterochromatin. Data on the evolution of sex chromosomes in Lepidoptera and their sister order Trichoptera suggest that a common ancestor of both orders had a Z/ZZ sex chromosome system. The $\mathrm{W}$ chromosome had evolved later, at a common branch of Tischeriina plus Ditrysia. During evolution of Ditrysia, derived sex chromosome systems had evolved such as neo-sex chromosomes, multiple sex chromosomes, and secondary $\mathrm{W}$ chromosome losses. Here we summarize our recent results on molecular differentiation of $\mathrm{W}$ chromosomes in selected species. Our main research tools were genomic in situ hybridization (GISH) with female-derived genomic probes, comparative genomic hybridization (CGH) with a probe mixture of differently labelled genomic DNA from females and males, FISH with probes prepared from $\mathrm{W}$ chromosome-derived bacterial artificial chromosome (W-BAC) clones of Bombyx mori, and FISH with W-painting probes prepared by laser microdissection of $\mathrm{W}$ chromatin followed by DOP-PCR amplification. Results suggest an accumulation of two types of repetitive sequences in $\mathrm{W}$ chromosomes: (i) repetitive DNA common to females and males, and (ii) W-specific repetitive DNA. Next the lack of cross-hybridization between Bombyx W-BACs and other lepidopterans reflects independent evolution of nonrecombining $\mathrm{W}$ chromosomes. Finally, a high specificity of W-painting probes denotes the advanced stage of molecular differentiation of $\mathrm{W}$ chromosomes.

\section{L106}

\section{In platypus a meiotic chain with five $X$ and five $\mathrm{Y}$ chromosomes links bird and mammal sex determination}

\author{
F. Grützner ${ }^{1}$, W. Rens ${ }^{2}$, E. Tsend-Ayush ${ }^{1}$, \\ N. El-Mogharbel ${ }^{1}$, P. O'Brien ${ }^{2}$, \\ M. Ferguson-Smith ${ }^{2}$, R. Jones ${ }^{3}$ and \\ J. Marshall Graves ${ }^{1}$
}

${ }^{1}$ Research School of Biological Sciences, Australian National University, ACT 2601, Australia; ${ }^{2}$ Centre for Veterinary Science, Department of Clinical Veterinary Medicine, University of Cambridge, UK; ${ }^{3}$ Department of Biological Sciences, The University of Newcastle, New South Wales 2308, Australia 
Two centuries after the discovery of the platypus, monotreme chromosome systems remain deeply puzzling. Karyotypes of male or both sexes were claimed to contain unpaired chromosomes (including the $\mathrm{X}$ ) that form a multivalent chain at meiosis. Other systems of translocation heterozygosity are known in plants and insects but not in vertebrates.

How the platypus chromosome system works to determine sex, and how balanced gametes and viable embryos are produced, has been controversial for 30 years. In order to decipher the complex chromosome system in platypus we generated chromosome paints for platypus chromosomes and hybridised them onto male and female metaphase chromosomes, meiotic cells and sperm. This showed that platypus has five malespecific chromosomes (Y), as well as five chromosomes present in one copy in males and two copies in females (X). These ten chromosomes form a multivalent chain at male meiosis, in which $\mathrm{X}$ and $\mathrm{Y}$ chromosomes alternate. the $\mathrm{X}$ and $\mathrm{Y}$ chromosomes segregate into two different types of sperm that determine female and male embryos respectively. An X chromosome with homology to the human $\mathrm{X}$ lies at one end of the chain, and a chromosome bearing the platypus homologue of DMRT1, the putative male determining gene in chickens, at the other.

This is the first evidence of a link between the mammalian and avian sex chromosome systems, which were previously thought to have evolved independently.

\section{L107}

\section{Exploring the kangaroo and platypus genomes}

\section{J. Marshall-Graves ${ }^{1}$}

${ }^{1}$ Research School of Biological Sciences, The Australian National University, Canberra, ACT, Australia

It makes excellent sense to include marsupial and monotreme species among mammal and other vertebrate species lined up for exhaustive genomic study. Comparisons between the more closely related eutherian species (eg human and mouse $\sim 70$ MY) often suffer because it is hard to distinguish signal from background noise, whereas in comparisons between vertebrate classes (eg human and chicken $\sim 310 \mathrm{MY}$ ) signal may be lost. Kangaroos (180 MY) and platypus (210 MY) occupy useful middle ground. Sequence has diverged sufficiently for stringent detection of homologies that can reveal coding regions and regulatory signals. Even more importantly, because marsupials and monotremes are mammals, they share with humans many mammal-specific developmental pathways and regulatory systems such as sex determination and $\mathrm{X}$ chromosome inactivation.

The major Australian research groups working on marsupial genetics have established a Centre for Kangaroo Genomics to gather resources, develop expertise and foster Australian and international collaborations for a serious onslaught on the genome of the model Australian kangaroo Macropus eugenii (the tammar wallaby). We are developing detailed physical and linkage maps of the genome to complement sequencing, and will prepare and array cDNAs for functional studies, especially of reproduction and development. At the same time, the Brazilian short-tailed opossum Monodelphis domestica is being fully sequenced by the NIH, so that it is possible to compare two very distantly related marsupials. We have also submitted a proposal to sequence the genome of the platypus, Ornithorhynchus anatinus, and this has received high priority for full sequencing by the NIH.

\section{PO:08:03}

\section{A post sex mismatched bone marrow FISH analysis showing an XXY, XXYY clone}

\author{
S. Engelbrecht ${ }^{1}$, M. Loubser ${ }^{2}$, J. Wakefield ${ }^{1}$, \\ S. Kolia ${ }^{1}$, L. Mogatusi ${ }^{1}$ and P. Willem ${ }^{3}$ \\ ${ }^{1}$ National Health Laboratory Service; ${ }^{2}$ Private \\ Practice at Olivedale Clinic Johannesburg; \\ ${ }^{3}$ National health Laboratory Service and \\ University of the Witwatersrand, South Africa
}

An eight-year old boy was diagnosed with the rare autosomal recessive disorder, mucpolysaccharidosis type VI. Bone marrow transplant (BMT) is a commonly accepted treatment for 
this syndrome. The patient received his first BMT in 1997, which was subsequently rejected. In August 2002 he received his second BMT from a blood type matched unknown female donor. A month post BMT the patient had Epstein-Barr virus (EBV) infection, which was treated with Retuximab. After an initial allergic reaction the patient recovered and was PCR negative for EBV infection. Fluorescent in situ hybridisation (FISH) was performed to determine chimerism following the sex mismatched BMT. The first FISH analysis using the commercially available probes from Vysis (CEP X, Xp11.1-q11.1 and CEP Y, Yq12) was performed by ourselves in March 2003. The analysis revealed $5 \%$ of cells displaying an XXY pattern. The same clone was still present in July 2003, along with an XXYY pattern. A full peripheral blood cytogenetic analysis was required and revealed a normal female karyotype. FISH analysis on the same specimen's metaphases showed the presence of Y-chromosome sequences on the telomeric end of the p-arm of the two Xchromosomes. In the latest FISH study on the patient's BM the clone was not present, but had $89.25 \%$ donor cells. We hope to conduct studies on the BM donor to establish if the XXY, XXYY cells are present. If this is not the case we postulate that the EBV infection might have played a role in the appearance of the aberrant clones.

\section{PO:08:04}

\section{Molecular analysis of microdissected W chromosomes of the codling moth, Cydia pomonella}

\section{Fukova ${ }^{1}$, M. Vitkova ${ }^{1}$, W. Traut ${ }^{2}$, S. Kubickova ${ }^{3}$ and F. Marec ${ }^{4}$}

${ }^{1}$ Faculty of Biological Sciences, University of South Bohemia, CZ-370 05 Ceske Budejovice, Czech Republic; ${ }^{2}$ Institute of Biology, Medical University, D-23538 Lubeck, Germany;

${ }^{3}$ Veterinary Research Institute, CZ-621 32 Brno, Czech Republic; ${ }^{4}$ Institute of Entomology ASCR, CZ-370 05 Ceske Budejovice, Czech Republic

The sex chromosome system in Lepidoptera is of the WZ type, and females are the heterogametic sex with a WZ sex-chromosome pair. Unlike Y chromosomes in $\mathrm{XY}$ systems very little is known about W chromosomes, and data about their molecular composition and actual role in sex determination are scarce. Here we present a detailed analysis of the $\mathrm{W}$ chromosome in the codling moth, Cydia pomonella (Tortricidae), the key pest of pome fruit in temperate regions of the world. The karyotype of codling moth consists of $2 \mathrm{n}=56$ chromosomes. In female meiosis, the $\mathrm{W}$ and $\mathrm{Z}$ chromosomes pair regularly in spite of the lack of apparent homology. The W consists largely of heterochromatin and is deeply stained with DAPI, indicating a high content of AT-base pairs. We studied molecular differentiation of the sex chromosomes by genomic in situ hybridization (GISH) and comparative genomic hybridization (CGH). GISH detected the W chromosome by strong binding of the Cy3-labelled, female-derived DNA probe. With $\mathrm{CGH}$, both the Cy3-labelled female-derived probe and Fluor-X labelled male-derived probe evenly bound to the $\mathrm{W}$. This suggested that the $\mathrm{W}$ is composed predominantly of repetitive DNA sequences occurring scattered in other chromosomes but accumulated in the W. Finally, we prepared W-specific probes by laser microdissection of the W chromatin followed by DOP-PCR and PCR labelling. The probes stained the $\mathrm{W}$ with a high specificity in a chromosome-painting manner. DNA fragments of the microdissected $\mathrm{W}$ chromatin were cloned and sequenced. The W-sequence analysis revealed no homology to any DNA sequenced so far.

\section{PO:08:05}

\section{The structure and evolution of sex chromosomes in Silene latifolia}

R. Hobza ${ }^{1}$, M. Lengerova ${ }^{2}$, E. Kejnovsky ${ }^{1}$, J. Siroky ${ }^{1}$, J. Macas ${ }^{3}$ and B. Vyskot ${ }^{1}$

${ }^{1}$ Institute of Biophysics, Czech Academy of Sciences, Brno, Czech Republic; ${ }^{2}$ Children's Medical Center, Brno, Czech Republic; ${ }^{3}$ Institute of Plant Molecular Biology, Czech Academy of Science, Ceske Budejovice, Czech Republic

Silene latifolia is the basic plant model to study sex determination and sex chromosome evolution. Here we present an improved FISH strategy for differentiating the sex chromosomes of 
Silene latifolia by chromosome painting. The $\mathrm{X}$ or $\mathrm{Y}$ chromosomes were isolated using nitrogen laser beam microdissection, catapulted by laser pressure, and amplified by DOP-PCR. We used a modified FAST-FISH protocol based on a short hybridization time combined with a low concentration of probe. The success of these experiments is demonstrated by the differential labeling of the $\mathrm{X}$ and $\mathrm{Y}$ chromosomes. This new approach represents a quick tool to compare organization of plant genomes. We have also generated new markers by constructing and screening a sample bacterial artificial chromosome (BAC) library for appropriate FISH probes. Five newly isolated BAC clones yielded discrete signals on the chromosomes: two were specific for one autosome pair and three hybridized preferentially to the sex chromosomes. We present the FISH hybridization patterns of these five BAC inserts together with previously described repetitive sequences (X-43.1, 25S rDNA and 5S rDNA) and use them to analyze the $S$. latifolia karyotype. Using one BAC insert and the three repetitive sequences, we have constructed a standard FISH karyotype that can be used to distinguish all autosome pairs. We have analyzed in detail the hybridization patterns of these sequences on the sex chromosomes. The results show that divergence of the sex chromosomes of S. latifolia is already in process and degeneration of the $\mathrm{Y}$ chromosome by accumulation of specific sequences has begun.

\section{PO:08:06}

\section{Yp terminal deletion in a 3-year old female patient with some stigmata of Turner's syndrome}

\author{
B. Kaluzewski ${ }^{1}$, M. Constantinou ${ }^{1}$, \\ Z. Helszer ${ }^{1}$, I. Plowas $^{1}$ and L. Korniszewski ${ }^{2}$ \\ ${ }^{1}$ Department of Medical Genetics, Medical \\ University, Lodz, Poland; ${ }^{2}$ Department of \\ Pediatrics, Section of Diabetology and Birth \\ Defects, Warsaw Medical Academy, Warsaw, \\ Poland
}

A child, delivered spontaneously from the first pregnancy on the 40th week from young unrelated parents. On the 12th week of pregnancy, a physician, while performing US examination, found extended nuchal translucency. Having consulted that observation with an obstetrician, the parents discontinued further prenatal diagnostics of the foetus. In delivery, oedema of feet and palms was noted. The psychomotor development of the child was normal. Periodical examination after the 2nd year of life did not reveal any major abnormalities, except a slight skrzydlowatosc of the neck and clearly visible podpaliczkowe areas in form of ãpouchesä. Preliminary cytogenetic examination, performed at a regional centre, revealed 46,X + mar karyotype. Another examination of the karyotype, performed at our Laboratory, verified the karyotype type into 46,XY. FISH technique with an SRY gene-specific probe enabled us to find deletion in the short arm of chromosome Y, involving the SRY gene. In molecular examination, employing PCR technique, aiming at determination of the extent of the described Yp deletion, it was found that in the patientâs karyotype, except the SRY gene, neither PABY nor ZFY loci occur, what cytogenetically confirms the loss of Yp11.3 band.

\section{PO:08:07}

\section{A novel structure associated with the chicken ZW lampbrush bivalent}

\section{A. Krasikova ${ }^{1}$, A. Saifitdinova ${ }^{1}$, S. Derjusheva ${ }^{1}$, S. Mizuno ${ }^{2}$ and E. Gaginskaya ${ }^{1}$}

${ }^{1}$ Biological Research Institute of Saint-Petersburg University, 198504, Russia; ${ }^{2}$ Department of Agricultural and Biological Chemistry, College of Bioresource Sciences, Nihon University, Fujisawa, Japan

In avian oocytes, sex chromosomes $\mathrm{Z}$ and $\mathrm{W}$ form an asymmetrical lampbrush bivalent with one chiasma, The $\mathrm{W}$ chromosome is strongly heterochromatic. Here we describe a spherical entity that forms in chicken germinal vesicles (GVs) in association with one of heterochromatic regions of $\mathrm{W}$ chromosome. By morphology, it resembles centromeric protein bodies (PBs) attached to lampbrush chromosome (LBCs) axis in Passerine birds and pigeons. In GVs, chicken $\mathrm{W}$ chromosome is known to consist of 7 chromomeres and 
to comprise XhoI, EcoRI and SspI highly repeated DNA sequences. Using FISH, we found that the spherical body associates with SspI repeat sequence, which localizes in non-centromeric chromomere 6 of $\mathrm{W}$ chromosome. In chaffinch GVs, PBs are known to form in association with centromeric FCP repeat. Using RTPCR we have verified that both SspI and FCP repeats transcribe during oogenesis, while EcoRI and XhoI repeats of chicken $\mathrm{W}$ chromosome do not. The sphere on chicken ZW bivalent does not stain by antibodies against phosphorilated form of RNA-polymerase II, while simple loops on Z lampbrush chromosome and small loops on the W brightly stain. Splicing snRNPs and SR-protein SC35 were not revealed in the sphere either. Since similar data are known for centromeric PBs on chaffinch and pigeon LBCs, the sphere on chicken $\mathrm{W}$ was identified with a kind of PBs. Unlike PBs in chaffinch and pigeon oocytes, the PB on chicken $\mathrm{W}$ does not stain by monoclonal antibodies 4A6 against DNA-topoisomerase II.

Supported by RFBR (02-04-49116), Russian Ministry of Education (PD02-1,4-291) and Science (MK-2655.2003.04).

\section{PO:08:08}

\section{Analysis of two different satellite DNA subfamilies in the complex sex-chromosome system of Rumex acetosa (Polygonaceae)}

\section{R. Navajas-Pérez ${ }^{1}$, T. Schwarzacher ${ }^{2}$, R. De La Herrán ${ }^{1}$, C. Ruiz Rejón ${ }^{1}$,} M. Ruiz Rejón ${ }^{1}$ and M. Garrido-Ramos ${ }^{1}$

${ }^{1}$ Departamento de Genética, Facultad de Ciencias, Universidad de Granada, Campus de Fuentenueva s/n, 18071, Granada, Spain; ${ }^{2}$ Department of Biology, University of Leicester. LE1 7RH, UK

Although satellite DNAs normally evolve concertedly, resulting in intra-specific sequence homogenization and inter-specific divergence, the existence of several mechanisms leading to satellite DNA subfamilies formation has been largely reported. Among the proposed causes, the existence of higher-order repeats, the population size and recombination disruptions are supposed to be the major factors.

Sex chromosomes represent good examples not only of repetitive sequence accumulation sites, but also of systems with an almost complete lack of recombination, and as such, they are ideal candidates for subfamily formation.

Specifically, in this study we analysed the sexchromosome system of the dioecious species Rumex acetosa (Polygonaceae) paying special attention to a Y-specific satellite DNA family, RAYSI. Rumex acetosa is a classical model in sex-determining mechanisms research due to the presence of a complex sex system comprising of females $2 \mathrm{n}=14(\mathrm{XX}+12$ autosomes) and males $2 \mathrm{n}=15$ (XY1Y2 + 12 autosomes). The two $\mathrm{Y}$ chromosomes appear not to recombine during male meiosis and both of them will only pair with the ends of each $\mathrm{X}$ chromosome arm. In this study, by means of molecular as well as fluorescent in situ hibridization techniques, we demonstrate the existence of two subfamilies of the RAYSI family (called RAYSI-S and RAYSI-J) looking at both fixed positions (homogenizated positions) and also their differential chromosome location. Causes and implications are discussed as well.

\section{PO:08:09}

\section{Reduced rates of sequence evolution of Y-linked satellite DNA in Rumex (Polygonaceae)}

\section{R. Navajas-Pérez ${ }^{1}$, R. De la Herrán ${ }^{1}$, M. Jamilena' ${ }^{2}$, R. Lozano ${ }^{2}$, C. Ruiz Rejón' ${ }^{1}$, M. Ruiz Rejón ${ }^{1}$ and M. Garrido-Ramos ${ }^{1}$}

${ }^{1}$ Departamento de Genética, Facultad de Ciencias, Universidad de Granada, 18071. Granada, SPAIN; ${ }^{2}$ Departamento de Biologia Aplicada, Escuela Técnica Superior de Ingenieros Agrónomos, Universidad de Almeria, Almeria, Spain

One characteristic feature of the sex chromosomes is the accumulation of a sort of repetitive DNA sequences in the $\mathrm{Y}$ chromosomes. However, little is known about how this occurs and about how the absence of recombination affects 
the subsequent evolutionary fate of the repetitive sequences in the $\mathrm{Y}$ chromosome. Here, we compare the evolutionary pathways leading to the accumulation of three families of satellite DNA sequences within the genomes of Rumex acetosa and Rumex papillaris, two dioecious plant species with a complex XX/XY1Y2 sexchromosome system. We have found that two of these families, one autosomic and one Y-linked, shared a common origin. Conversely, they are not related in origin with the third one, also located in the $\mathrm{Y}$ chromosomes. However, we have demonstrated that the two satellite DNA families in the $\mathrm{Y}$ chromosomes of these species have reduced rates of evolution and sequence homogenization in relation to those found for the satellite DNA in autosomes.

\section{PO:08:10}

\section{Cytogenetic and moleculargenetic screening of fragile $X$ syndrome}

\author{
S. Padma ${ }^{1}$, K. R. Manjunatha ${ }^{1}$, \\ G. K. Chetan ${ }^{1}$, S. R. Girimaji*1, S. Roy ${ }^{1}$, \\ E. Venkataswamy ${ }^{1}$, S. Balu1 and \\ H. N. Venkatesh ${ }^{1}$
}

${ }^{1}$ Departments of Human Genetics \& Psychiatry*, National Institute of Mental Health \&

Neurosciences (NIMHANS), Bangalore 560029, India

Fragile $\mathrm{X}$ syndrome represents the most common inherited cause of mental retardation. The key clinical features of the fragile $\mathrm{X}$-syndrome in males are mental retardation, elongated triangular face, lop ears and macro-orchidism. The fragile $\mathrm{X}$ syndrome was the first disease shown to be associated with 'dynamic mutations', caused by an amplification of an unstable CGG repeat sequence located at the $5^{\prime}$ untranslated region of FMR1 gene. A fragile site at the distal long arm of the X chromosome (FraXq 27.3) is the hallmark cytogenetic feature of the syndrome. AIM:This study was aimed to facilitate both cytogenetic and molecular screening of fragile $\mathrm{X}$ syndrome in Indian population for the analysis of the mutation.

Fifty subjects with fragile $\mathrm{X}$ syndrome clinical features, were studied. Cytogenetic and molecular analysis using lymphocyte culture, PCR and Southern technique, were done. The PCR method provided rapid and reliable results for the identification of fragile $\mathrm{X}$ negative and positive patients. The PCR-positive case was confirmed by the CGG repeat expansion on Southern blot analysis with a positive cytogenetic result. Our conclusions are that cytogenetic and molecular genetic techniques improve diagnostic accuracy and allow verification of the normal condition of FRAXA locus, identification of carriers and detection of complete mutations in fragile $\mathrm{X}$ syndrome and, ultimately, proper genetic counselling in fragile $\mathrm{X}$ families. With fragile $\mathrm{X}$ syndrome being such a complex condition, national screening would need to be considered for families before a child is conceived so that an informed choice can be made.

\section{PO:08:11}

\section{Nuclear spacing in the syncytial blastoderm of Drosophila melanogaster embryos}

\author{
S. Pichler ${ }^{1}$, T. Gregor ${ }^{2}$ and D. Glover ${ }^{1}$ \\ ${ }^{1}$ Department of Genetics, Cambridge University, \\ Cambridge CB2 3EH, UK; ${ }^{2}$ Department of \\ Molecular Biology, Princeton University, \\ Princeton, New Jersey, 08544, USA
}

In D. melanogaster, inhomogeneities in the spacing of nuclei along the A-P axis are first visible in cycle 11 of the syncytial blastoderm embryo (Blankenship and Wieschaus, 2001). In the anterior domain, spaces between nuclei are expanded, in the pre-cephalic furrow domain, nuclear distances are the smallest, and in the posterior domain, nuclear distances are intermediate. Nuclear spacing represents the earliest manifestation of zygotic transcription mediated by bicoid. Alpha-amanitin injections inhibit zygotic transcription and nuclear spacing is established in uniform manner along the A-P axis. We have developed Mathlab programmes which measure nuclear distances and densities to identify the genes and mechanisms that regulate nuclear spacing before cellularization. Embryos lacking the $\mathrm{X}$-chromosome do not form the anterior domain 
with expanded nuclear spacing. Genetic mapping places the gene required for nuclear spacing in region $7 \mathrm{~A}-7 \mathrm{~B}$. I am genetically fine-mapping the region and setting up RNAi experiments to identify the gene on the X-chromosome involved in nuclear spacing.

I am investigating nuclear spacing in bcd;nos; tl, nos;bcd and osk embryos to investigate a potential function of maternal protein gradients in nuclear spacing and in mutants of the gap genes giant, knirps and even-skipped.

\section{PO:08:12}

\section{Cytogenetic and molecular characterization of an isodicentric $\mathrm{Y}$-chromosome in an infertile man}

\author{
A. Veble ${ }^{1}$, K. Writzl ${ }^{1}$, B. Zorn ${ }^{2}$ and \\ B. Peterlin'
}

${ }^{1}$ Division of Medical Genetics, Department of Obstetrics and Gynecology, UMC Ljubljana; Ljubljana, Slovenia; ${ }^{2}$ Andrology Centre, Department of Obstetrics and Gynecology, UMC Ljubljana; Ljubljana, Slovenia

One of the most common structural changes of the $\mathrm{Y}$ chromosome is dicentrism. The phenotype of patients with a karyotype $45, \mathrm{X} / 46, \mathrm{X}, \operatorname{idic}(\mathrm{Y})$ range from almost normal males through mixed gonadal dysgenesis to females with Turner syndrome phenotype.

We present a 29-year-old man who was referred to cytogenetic assessment because of azoospermia. He had testicular atrophy (left and right testicular volumes were $7 \mathrm{ml}$ ); histopathologic analysis of testis biopsy demonstrated maturation arrest. The GTG- and CBG-banded karyotype showed two cell lines, one of them contained isodicentric Y [idic(Yp)] chromosome, which was seen in $76 \%$ of metaphases analysed, and a $45, \mathrm{X}$ cell line $(24 \%)$. This was confirmed by FISH analysis with CEP Y, ptel Y and qtel Y probes. For precise characterisation of the Yq breakpoint, PCR analysis using primers for six markers loci (sY84, sY127, RBMY1, sY152, sY147 and sY254) was performed. The deletion of $\mathrm{AZFb}$ and $\mathrm{AZFc}$ region was confirmed.
This clinical report could be helpful in defining the phenotypic range associated with mosaic $\mathrm{Y}$ isodicentrics.

\section{PO:08:13}

\section{Markers for uncovering the evolution of sex chromosomes in Lepidoptera}

\author{
M. Vitkova ${ }^{1}$, I. Fukova ${ }^{2}$ and F. Marec ${ }^{2}$ \\ ${ }^{1}$ Faculty of Biological Sciences, Univerzity of \\ South Bohemia, CZ-370 05 Ceske Budejovice, \\ Czech Republic; ${ }^{2}$ Institute of Entomology ASCR, \\ CZ-370 05 Ceske Budejovice, Czech Republic
}

Most insects possess Drosophila/mammalian-type sex chromosome systems, XX/XY or derived variants, with males being the heterogametic sex. Female heterogamety occurs only in two insect orders, the Trichoptera (caddis flies) and Lepidoptera (moths and butterflies), both orders forming a common clade, the superorder Amphiesmenoptera. Trichoptera and primitive Lepidoptera possess a $\mathrm{Z} / \mathrm{ZZ}$ system. Whereas a $\mathrm{WZ} / \mathrm{ZZ}$ system is common in advanced Lepidoptera, and numerical variations of the WZ type are known as well. Here we examined several lepidopteran species from primitive ones, which lack the $\mathrm{W}$ chromosome, to advanced ones with well-differentiated $\mathrm{W}$, in order to uncover the evolutionary history of their sex chromosomes. The use of genomic in situ hybridization (GISH) and comparative genomic hybridization (CGH) enabled us to identify the female-determining $\mathrm{W}$ chromosomes and define the level of their molecular differentiation from the $\mathrm{Z}$ chromosomes. Potential homology between sex chromosomes of different species was tested with the help of several sex-chromosome markers such as, for example, the MBSAT1 satellite recently discovered in Mamestra brassicae, perZ gene from the $\mathrm{Z}$ chromosome in Antheraea pernyi, and Bmkettin gene found on the $\mathrm{Z}$ chromosome of Bombyx mori. The presence of selected markers in the genome of a particular species was examined by Southern hybridization. Then the markers were localized on chromosomes by FISH. Results achieved enabled us to restate the hypothesis on the evolution of lepidopteran sex chromosomes. 
PO:08:14

\section{Detection of sex chromosome constitution by GISH and telomere-FISH in some Lepidoptera}

\author{
A. Yoshido ${ }^{1}$, K. Sahara ${ }^{1}$, Y. Yasukochi ${ }^{2}$ \\ and F. Marec ${ }^{3}$ \\ ${ }^{1}$ Graduate School of Agriculture, Hokkaido \\ University, Japan; ${ }^{2}$ Genome Research \\ Department, National Institute of \\ Agrobiological Sciences, Japan; ${ }^{3}$ Institute of \\ Entomology, Czech Academy of Sciences, \\ Czech Republic
}

Sex chromosomes are designated $\mathrm{X}$ and $\mathrm{Y}$ in systems with male heterogamety and $\mathrm{W}$ and $\mathrm{Z}$ in those with female heterogamety. In species with multiple sex chromosomes, it is sometimes difficult to disclose the actual sex chromosome constitution. Examination of several specimens with various chromosome stages is inevitable to get the definitive conclusion in such cases. One must conventionally compare the number of chromosomes between females and males, and analyze chromosome pairing, the synaptonemal complex formation and/or chiasma formation, if adequate molecular markers of sex chromosomes are not available.

Moths and butterflies (Lepidoptera) exemplify the female heterogamety with a WZ/ZZ sex chromosome system or its numerical variations (e.g., Z/ZZ, W1W2Z/ZZ, WZ1Z2/Z1Z1Z2Z2). Вy using combination of GISH and telomere-FISH, we have developed a simple method to identify the sex chromosome constitution in Lepidoptera. GISH highlighted the heterochromatin of $\mathrm{W}$ chromosomes and PCR-generated telomeric repeat probes, (TTAGG)n, detected chromosome ends. This approach enabled us to acquire gross but reliable information about sex chromosomes from a single-specimen preparation of pachytene oocytes. Another merit of this method consists in its universal use for any species with molecularly differentiated sex chromosomes. With this method we showed that Samia cynthia pryeri has a WZ1Z2 sex chromosome constitution, whereas the subspecies $S$. c. ricini has a $\mathrm{Z} / \mathrm{ZZ}$ sex chromosome system. The $\mathrm{W}$ chromosome in $S$. c. pryeri presumably consists of 3 parts, an original
W-heterochromatin segment and two segments translocated onto the $\mathrm{W}$ chromosome from autosomes. The sex chromosome evolution in Samia cynthia is discussed.

\section{Chromosome Transfer Symposium}

\section{L108}

\section{Tumorigenicity suppressor genes - their history as a prime example of bias in science}

\section{H. Klinger ${ }^{1}$}

${ }^{1}$ Albert Einstein College of Medicine, NY, USA

No abstract was submitted for this talk.

\section{L109}

\section{Chromosome engineering using chromosome transfer for functional analyses}

\author{
M. Oshimura ${ }^{1}$ \\ ${ }^{1}$ Department of Biomedical Science, Institute of \\ Regenerative Medicine and Biofunction, \\ Graduate School of Medical Science, Tottori \\ University, Yonago, Tottori 683-8503, Japan
}

The method of microcell-mediated chromosome transfer is important for mapping genes to specific chromosome when the gene has a specific function. For example, we have used this method to map genes involved in tumor suppression, cellular aging, metastasis, DNA repair and genomic imprinting. More recently, we have established a novel procedure to introduce foreign genetic material into mice by using the chromosome itself as a vector and provided the first examples of transchromosomic mice containing a heritable foreign chromosome. Application of this procedure was demonstrated on creations of the mice expressing fully human antibodies and Down syndrome model mice with an extra human chromosome 21. For more precise mapping, functional studies, making TC mice and human artificial chromosome, we are currently engineering human 
chromosomes in the chicken DT40 cells with a high homologous recombination rate. For this pourpose, the human chromosome containing a locus of interest is modified by chromosome truncation at the integration site of the telomeric repeat or by the integration of a target construct by homologous recombination. In this talk, lessons learned from our studies using the chromosome engineering will be introduced.

\section{References}

1) Tomizuka et al. Functional expression and germline transmission of a human chromosome in chimeric mice. Nature Genet., 16:133, 1997

2) Kuroiwa et al., Manipulation of human minichromosomes to carry greater than megabase-sized chromosome inserts. Nat. Biotechnol., 18:1086, 2000.

3) Horike et al., Targeted disruption of the human LIT1 locus defines a putative imprinting control element playing an essential role in Beckwith-Wiedemann syndrome. Hum. Mol. Genet., 9:2075, 2000.

\section{L110}

\section{Tumour suppressor genes in malignant melanoma}

\section{Parris}

Brunel University, UK

Deletions involving regions of chromosomes 1,6 , 9 and 10 are frequent events in the development and progression of cutaneous malignant melanoma (CMM). To investigate the possibility that these chromosomes encode important tumor suppressor genes, we introduced normal copies of chromosomes 1,6 and 10 to the tumorigenic human metastatic melanoma cell line UACC-903 using microcell mediated chromosome transfer. In addition, two chromosome 9 variants microdeleted for the p16 and p15 loci were also transferred to determine whether an additional melanoma tumor suppressor gene (TSG) resides on chromosome 9 independent of these loci. Chromosome 1 hybrids failed to show consistent suppression of growth in soft agar or reduced tumour development in nude mice. The resulting microcell hybrids of the chromosomes $6,9 \Delta \mathrm{p} 16 / \mathrm{p} 15$ variants and 10 were significantly less tumori- genic than the parental UACC-903 cell and control hybrids of chromosomes 9(q-arm), 5 and 15, as judged by their ability to form colonies in soft agar and tumors in athymic female $n u / n u$ mice. The data presented here defines additional TSG(s) on chromosome 9 independent of the p16/p15 tumor suppressor genes and provides strong evidence for the presence of a TSG(s) central to CMM development on chromosome 10.

\section{L111}

\section{The role of the nuclear envelope in chromosome positioning: using monochromosome hybrids cells as a model system}

\author{
K. Meaburn ${ }^{1}$, H. Cox ${ }^{1}$, J. Ellis ${ }^{2}$, R. Newbold ${ }^{3}$ \\ and J. Bridger ${ }^{1}$
}

${ }^{1}$ Lab. of Nuclear and Genomic Health, Cell and Chromosome Biology Group, Dept Biological Sciences, Brunel University, Uxbridge, Middlesex, UB8 3PH, UK; ${ }^{2}$ Randall Centre for the Molecular Mechanism of Cell Function, Kings College, London University, Guys Campus, London, SE1 1UL, UK; ${ }^{3}$ Institute of Cancer Genetics and Pharmacogenomics, Dept Biological Sciences, Brunel University, Uxbridge, Middlesex, UB8 3PH, UK

Specific human chromosomes are found reproducibly at the nuclear periphery in proliferating cells. There are a number of inner nuclear envelope (INE) proteins that may have roles in genome organisation and location in interphase nuclei; examples of such proteins are emerin and A-type lamins. Mutations in the genes of either can result in X-linked EDMD or a wide range of diseases collectively termed laminopathies, respectively. We find that specific chromosomes normally located at the nuclear periphery, in normal proliferating human dermal fibroblasts (HDF) are located more internally in proliferating HDF derived from patients with mutations in the A-type lamin or emerin gene. The emerin mutant cell line we have studied lacks both emerin and lamin C.

In order to further study the role of INE proteins in targeting chromosomes to the nuclear periphery, we have employed a model cell system, in 
which stable hybrid cell lines carry a single, intact human chromosome in immortalised mouse fibroblasts. We have found that the position of most human chromosomes normally found at the nuclear periphery has altered. We are testing the role of various human INE proteins in the positioning of selected human chromosomes by stable transfection of INE containing constructs into these cells. Our data so far suggests that, in this hybrid system, Lamin A alone is not critical for positioning chromosomes at the nuclear periphery. Taken together, these data imply a role for lamin $\mathrm{C}$ in the positioning of human chromosomes to the nuclear periphery.

\section{PO:08:15}

\section{Reciprocal translocations detected at prenatal diagnosis: results from 5 years experience}

\author{
R. Pinto Leite ${ }^{1}$, B. Carvalho ${ }^{1}$, M. Souto ${ }^{1}$ \\ and E. Ribeiro ${ }^{1}$
}

${ }^{1}$ Centro Hospitalar de Vila Real-Peso

da Régua, SA

Reciprocal translocations are not uncommon, arise 1 in 625 new born of the general population and once in every 1000 amniocentesis. It is urgent to do parental chromosomal studies, to distinguish between a familiar or a de novo rearrangement in the fetus, to predict the risk for physical malformation and mental deficiency; that is approximately 10 percent when it is a de novo situation.

A total of five foetuses with reciprocal translocations, 1 de novo and 4 familiar, were detected at prenatal diagnosis, during July 1998 through December 2003 at the Centro de Diagnostico Prenatal of the Centro Hospitalar de Vila Real-Peso da Régua, S.A. Using conventional cytogenetics and fluorescence in situ hybridization (FISH) we characterized the rearrangements. Amniocenteses were performed because of maternal age (2 cases); positive biochemical screening for trisomy 21 ( 2 cases) and one familiar case of reciprocal translocation. The karyotypes were interpreted as $46, \mathrm{XX}, \mathrm{t} \quad(3 ; 5) \quad(\mathrm{q} 25 ; \mathrm{q} 13) \quad ; \quad 46, \mathrm{XY}, \mathrm{t} \quad(4 ; 7)$ (q21;p15)pat ; 46,XX,t $(2 ; 14)(\mathrm{p} 25.1 ; \mathrm{q} 13.1) \mathrm{mat}$;
46,XY,t $\quad(3 ; 15) \quad(\mathrm{p} 21 ; \mathrm{q} 24)$ pat $; 46, \mathrm{XX}, \mathrm{t} \quad(3 ; 13)$ (q23;q22)mat. The parents decided to continue the pregnancies in all the cases. The clinical significance of de novo reciprocal translocation is a major problem in prenatal counselling. Molecular cytogenetics tools such as FISH are indispensable for characterizing the breakpoints involved in the reciprocal translocation.

\section{PO:08:16}

\section{De novo partial duplication of chromosome $7 p$ at a prenatal diagnosis}

\author{
M. Souto ${ }^{1}$, R. Pinto Leite ${ }^{1}$, B. Carvalho ${ }^{1}$ \\ and E. Ribeiro ${ }^{1}$ \\ ${ }^{1}$ Centro Hospitalar de Vila Real-Peso \\ da Régua, SA
}

Partial duplications of the short arm of chromosome 7 are a rare occurrence. The most common mechanism leading to $7 \mathrm{p}$ duplication is unbalanced segregation of a parental reciprocal translocation at meiosis.

We report a case, detected in a prenatal diagnosis that has an extra segment on the proximal short arm of chromosome 7.

A 34 year-old pregnant woman was referred for amniocentesis in the 16th week of pregnancy because of ultrasound anomalies. The couple had an obstetric history of 5 miscarriages and 3 healthy children.

The GTG analysis revealed an extra segment on the proximal short arm of chromosome 7 in two different cultures. Parent's karyotypes were normal. In order to clarify the chromosome origin of this extra segment, chromosome painting was done. Fluoresecence in situ hybridization (FISH) using a whole chromosome 7 DNA probe (Cytocell), showed that the extra chromosome material is derived from chromosome 7, confirming that the foetus is partially trisomic for chromosome 7 . The exclusion of a balanced reciprocal translocation at one of the parents suggests the presence of a gonadal mosaicism.

When the couple came to prenatal counselling, to explain the implications of this result, the ultrasound detected in utero death.

A bibliographic review is presented. 


\section{Gene Therapy Symposium}

\section{L112}

\section{Mammalian artificial chromosomes (MACs) as gene therapy vectors}

C. Huxley ${ }^{1}$, G. Kotzamanis ${ }^{1}$, W. Cheung ${ }^{1}$, H. Abdulrazzak ${ }^{1}$ and S. Perez-Luz ${ }^{1}$

${ }^{1}$ Imperial College, London, UK

A Mammalian Artificial Chromosome (MAC) vector should carry all the functional elements of a chromosome (centromere, telomeres and replication origins) to allow the DNA to be maintained as an independent replicating and segregating molecule in mammalian cells. MACs carrying large genomic regions are potentially useful gene therapy vectors as they should combine long-term stable maintenance with full levels of tissue specific and controlled expression from the large DNA inserts. As MACs carry no viral DNA there should be few immunological problems and as they are maintained as episomes there is little danger of mutagenesis due to integration.

We have utilized homologous recombination in E. coli using the Red genes to recombine BACs carrying large genomic regions and alphoid DNA into a single BAC vector which can form MACs when transfected into HT1080 cells. We have also developed a system using Red recombination which allows one to link overlapping BACs and have used this system to recombine three overlapping BACs covering the entire CFTR gene with all its regulatory elements into a single BAC clone, and also to recombine two overlapping BACs covering the entire Factor VIII gene into a single $\mathrm{BAC}$. We are using these $\mathrm{BAC} / \mathrm{MAC}$ vectors to study methods for delivery of large DNA into cells in vitro and in vivo, MAC formation in a variety of cell lines and gene expression from MAC vectors.

\section{L113}

\section{Approaches to genetic therapy for neuromuscular and cardiovascular diseases: gene addition and gene correction strategies}

\author{
G. Dickson ${ }^{1}$
}

${ }^{1}$ School of Biological Science, Royal Holloway - University of London, Egham, Surrey, TW20 0EX, UK

A number of approaches are under development to provide genetic therapies based upon the concepts of (i) gene addition to complement inherited genetic deficiencies, and (ii) gene correction to repair endogenous mutations. In the case of gene addition therapy, a range recombinant viral and non-viral vector systems have been adapted to transfer genes to human and animal cells. Conversely, for gene correction strategies, the most successful approaches have involved the use of synthetic and chemically-modified oligonuceotides to either target base changes in specific genes, or to modulate gene expression at transcriptional and post-transcriptional levels. Recent advances in both areas will be described in the context of two disease scenarios: firstly the rare inherited neuromuscular disease, Duchenne muscular dystrophy, and secondly in the context of atherosclerosis, a common disorder which represents a healthcare issue of virtual epidemic proportions, and for which no effective non-invasive therapy exists. In particular, specific case studies will be presented in relation to preclinical investigations with experimental animal models.

\section{L114}

\section{DNA and chromatin structures required for de novo formation of a human artificial chromosome}

\author{
H. Masumoto ${ }^{1}$, H. Nakashima ${ }^{2}$, M. Nakano ${ }^{1}$ \\ and J. Ohzeki ${ }^{1}$ \\ ${ }^{1}$ LBC, NCI/NIH, Bldg37, Rm5040, 9000 \\ Rockville Pike, Bethesda, MD 20892, USA; \\ ${ }^{2}$ Graduate School of Science, Nagoya Univ., \\ Nagoya 464-8602, Japan
}

HAC formation assays with mutation analyses indicated an obvious requirement of alphasatellite (alphoid) DNA and functional CENP$B$ boxes reflecting human normal centromere DNA configurations for de novo centromere assembly. However, this de novo-created HAC has some unsolved problems. All the alphoid YAC/BAC DNA introduced into cultured 
human cells were multimerized. A strong transcriptional activity from a gene on the YAC arms was conflicted with the HAC formation. Before applying the HAC as a therapeutic gene delivery vector, it is necessary to overcome these unsolved problems. The first-generation HACs still have a crucial advantage for identifying the important as yet undefined property or structure required for a stable human chromosome because they consist entirely of introduced DNA molecules. Our data obtained from analyses of the chromatin structures on the stable HAC and on ectopic alphoid YAC integration sites indicate that the active state of the centromere is generated and/or maintained in association with an open chromatin structure on the alphoid YAC multimer, and the inactive state of centromere is rather linked with a silent and suppressed structure like heterochromatin. And, either an active or inactive state of the centromere can be converted by a structural change in the chromatin on type-I alphoid DNA. I also discuss the effect of the strong transcription. For the efficient de novo centromere assembly and HAC formation it might be necessary to control such a delicate balance between open chromatin formation on the introduced naked type-I alphoid DNA and heterochromatinization pressure on the naked DNA.

\section{L115}

\section{Establishing artificial chromosomes in human and murine cells}

\section{Z. Larin Monaco ${ }^{1}$}

${ }^{1}$ Weatherall Institute of Molecular Medicine, University of Oxford, John Radcliffe Hospital, Oxford OX3 9DS, UK

Human artificial chromosomes (HACs) are autonomous molecules that can function and segregate as normal chromosomes in human cells. They are generated as newly formed (de novo) artificial chromosomes in human cells following the introduction of vectors containing human alpha satellite (alphoid) DNA, which is the primary sequence present at all human centromeres. We are developing artificial chromosomes for gene expression studies in human and murine cells.
Artificial chromosomes containing the HPRT gene, were used successfully in our group to complement the defect in human HT1080 HPRT- deficiency cells. We are investigating a system for efficient delivery of HAC vectors to other cell types based on the Herpes Simplex Virus-1 amplicon technology. We are also establishing artificial chromosomes in murine cells to develop novel gene expression vectors which will be important for investigation of human transgene expression in murine models of human genetic diseases.

\section{L116}

\section{Assaying centromere assembly using engineered mini-chromosomes and novel site-specific recombinases}

\author{
W. Brown ${ }^{1}$, F. Dafhnis-Calas ${ }^{1}$, S. Malla ${ }^{1}$, \\ Z. $\mathrm{Xu}^{1}$ and $\mathrm{M}$. Smith ${ }^{2}$ \\ ${ }^{1}$ Institute of Genetics, Queens Medical Centre, \\ Nottingham University, NG7 2UH, UK; \\ ${ }^{2}$ Institute of Medical Sciences, University of \\ Aberdeen, Foresterhill, Aberdeen, AB25 2ZD, \\ Scotland, UK
}

We discovered that engineered human mini-chromosomes with limiting amounts of centromeric DNA assemble centromeres in chicken and human cells but fail to do so in mouse cells where consequently they segregate inaccurately. This observation is important because it provides the basis of an assay for the ability of a piece of DNA to be assembled into a centromere. In order to exploit this potential we have developed a new set of techniques and reagents that allow us to build and swap centromeres on mini-chromosomes. Our techniques enable the assembly and replacement of long tracts $(>150 \mathrm{~kb}$ ) of DNA of defined sequence organization on mini-chromosomes.

\section{L117}

\section{Characterization of euchromatin and heterochromatin on human artificial chromosomes}
B. Grimes', J. Babcock' ${ }^{2}$, M. Rudd ${ }^{3}$, B. Chadwick ${ }^{3}$ and H. Willard ${ }^{3}$ 
${ }^{1}$ Department of Medical and Molecular Genetics, Indiana University School of Medicine, $975 \mathrm{~W}$. Walnut St, IB130, Indianapolis, IN 46202, USA.; ${ }^{2}$ Department of Genetics, Center for Human Genetics, Case Western Reserve University School of Medicine and University Hospitals of Cleveland, Cleveland, OH 44106, USA.; ${ }^{3}$ Institute for Genome Sciences \& Policy and Department of Molecular Genetics and Microbiology, Duke University, Durham, NC 27710, USA

Formation of euchromatin on artificial chromosomes is considered important for creating a suitable chromosome environment for gene expression in gene transfer applications. In addition, deposition of heterochromatin on artificial chromosomes may be important for centromere function. To evaluate the chromatin composition of human artificial chromosomes, we have used indirect immunofluorescence on metaphase spreads to characterize markers of either euchromatin (H3DimK4 modified nucleosomes) or heterochromatin (H3MeK9 modified nucleosomes and HP1-alpha) on a panel of human artificial chromosomes derived from higher-order repeat alpha satellite from chromosomes X or 17. Whilst all artificial chromosomes assembled euchromatin markers, deposition of heterochromatin was variable. Larger artificial chromosomes $(3-10 \mathrm{Mb})$ were enriched for heterochromatin and replicated later in $\mathrm{S}$ phase while smaller artificial chromosomes $(<3 \mathrm{Mb})$ were depleted for heterochromatin, largely euchromatic and replicated earlier in $\mathrm{S}$ phase.

Since these data suggest that the chromatin environment on artificial chromosomes is likely to be broadly permissive for gene expression, they lend further support for the proposed development of artificial chromosomes as gene delivery vectors. Further, it appears that heterochromatin can be entirely dispensible or required only in small amounts for human centromere function.

\section{L118}

\section{Development of episomal vectors carrying large genomic regions for gene therapy}

\author{
W. Cheung ${ }^{1}$, H. Abdulrazzak' ${ }^{1}$, G. Kotzamanis ${ }^{1}$ \\ and C. Huxley ${ }^{1}$
}

${ }^{1}$ Division of Biomedical Sciences and MRC Clinical Sciences Centre, Imperial College, London, UK

For gene therapy to be effective in the treatment of human diseases, vectors with sustained levels of gene expression over long periods of time are required. The aim here is to develop vectors carrying large genomic loci that are maintained as episomes in cells. Entire genomic loci possessing all the long-range controlling elements of a gene should confer tissue-specific and regulated expression. Episomal vectors should allow longterm maintenance of the DNA, even in dividing cells, without the hazard of integration.

The oriP and EBNA-1 components from Epstein-Barr virus allow efficient maintenance of DNA as multicopy episomes in human and mouse cells. A 170-kb BAC vector carrying the intact human HPRT genomic locus and oriP/EBNA-1 was transfected into mouse $\mathrm{B} 16 \mathrm{~F} 10$ cells and stable cell lines established. The BAC was maintained as an intact, unrearranged episome in about half of the cell lines and expression of human HPRT was largely proportional to copy number.

Alternatively, vectors with centromeric alphoid DNA from mammalian chromosomes can be maintained as artificial chromosomes following de novo centromere formation. A BAC carrying the entire HPRT locus and $70 \mathrm{~kb}$ of alphoid DNA was transfected into HPRT-negative HT1080 cells and formed a de novo mammalian artificial chromosome in 2 of the 14 cell lines analysed. Both cell lines expressed HPRT and grew in the presence of HAT selection.

We are continuing to investigate the relative merits of oriP/EBNA-1 and MAC vectors carrying large genomic loci as gene therapy vectors.

\section{L119}

\section{The ACE system: a chromosome engineering and delivery technology for cell-based gene therapy}

C. Perez ${ }^{1}$, S. Vanderbyl, S. Stewart, N. Macdonald, T. Stodola, K. Mills, E. Lee, G. Lee, A. Telenius and H. Ledebur, Jr.

${ }^{1}$ Chromos Molecular Systems, Inc., Burnaby, BC, Canada V5A 1W9 
The Artificial Chromosome Expression [ACE] System is a versatile, reliable technology for the rapid generation of mammalian cells/cell lines expressing heterologous genes. These cells are candidates for cell-based gene therapy and/or recombinant protein production at industryrelevant levels. The ACE System consists of a mammalian artificial chromosome engineered to contain multiple integration sequences for sitespecific recombination, a targeting vector that recombines onto the chromosome at the integration sites to load the gene of interest, and a proprietary plasmid-encoded integrase that is cotransfected to catalyze integration. ACE chromosomes [ACEs] are stably maintained, non-integrating, autonomously replicating, and easily isolated by flow cytometry. They have been transfected with standard reagents into a variety of cell types, including adult human mesenchymal stem cells (hMSCs), and have been microinjected into fertilized oocytes to generate transgenic animals.

Using the ACE system we generated $\mathrm{CHO}$ and LMtk- cells secreting human erythropoietin [EPO], each of which were subcutaneously implanted into SCID mice. Hematocrits were measured for 2-3 weeks post-implantation, and demonstrated therapeutically significant increases in 8 mice relative to mice with control cells carrying unloaded ACEs. Recently we have engineered an ACE encoding both green fluorescent protein [GFP] and EPO. It was purified and transfected into hMSCs. The resulting population was enriched to $20 \%$ GFP-positive cells, which secreted EPO at a rate of 50-100 IU/million cells/day. EPO expression at this level is comparable to what can be attained in hMSCs with retroviral vectors, suggesting that the ACE System is a novel versatile technology for implementing cellöbased gene therapy.

\section{PO:08:17}

\section{The formation of de novo artificial chromosomes in mammalian cells}

\author{
H. Abdulrazzak ${ }^{1}$, W. Chung ${ }^{1}$, G. Kotzamanis ${ }^{1}$ \\ and C. Huxley \\ ${ }^{1}$ Division of Biomedical Sciences and MRC \\ Clinical Sciences Centre, Imperial College \\ School of Science Technology and Medicine, \\ London, UK
}

Mammalian artificial chromosomes (MACs) are useful not only for allowing basic research into the requirements for mammalian chromosome function and stability during cell division but may serve to provide a vector for the introduction of individual genes or a cluster of genes into recipient cells. It has been shown that the introduction of cloned human alphoid DNA into the human fibrosarcoma cell line HT1080 is sufficient for formation of de novo MACs.

HPRT-alphoidNELBAC is a construct that we generated which contains the HPRT gene, $70 \mathrm{~kb}$ of alphoid DNA as well as a neomycin resistance and enhanced fluorescent green protein genes. The construct was transfected into HPRTnegative HT1080 cells and formed a de novo MAC in 2 of the 14 cell lines analysed. The construct was also introduced into the mouse cell line, B16F10. Nine drug resistant clones were analysed and found to contain genomic integration of the DNA. In one of these clones, the construct integrated in a small percentage of cells. The remainder of the cells contained multiple episomally maintained structures. These were rapidly lost upon propagation of the cells in the absence of selection.

A second construct (70alphoidNELBAC) has been generated through partial digestion which lacks the HPRT gene but retains the other elements. This construct will be introduced into HPRT-negative HT1080 in parallel with HPRTalphoidNELBAC to assess the influence of the HPRT gene on MAC formation. The construct will also be introduced into a variety of mammalian cells to assess their ability to form de novo MACs.

\section{PO:08:18}

\section{Construction of a novel human artificial chromosome (HAC) and its use for gene delivery}

\author{
M. Katoh ${ }^{1}$, F. Ayabe ${ }^{2}$, S. Norikane ${ }^{3}$, \\ H. Hoshiya ${ }^{2}$, X. Ren ${ }^{2}$, T. Suda ${ }^{1}$, C. Okita ${ }^{2}$ \\ and M. Oshimura ${ }^{2}$ \\ ${ }^{1}$ Department of Human Genome Science, \\ Graduate School of Medical Science, Tottori \\ University, Japan; ${ }^{2}$ Department of Biomedical \\ Science, Institute of Regenerative Medicine and \\ Biofunction, Graduate School of Medical Science,
}


Tottori University, Japan; ${ }^{3}$ Department of Molecular and Cell Genetics, Graduate School of Medical Science, Tottori University, Japan

Ex vivo gene delivery and auto transplantation is a way for gene therapy. Prerequisites for vectors are 1) long term stable maintenance in host cells, 2) no risk for disrupting host genome by integration and 3) being under physiological regulation of host cells. Human chromosome fragment (hCF) is transferable from donor to recipient cells by microcell fusion in vitro. Transfer of hCFs to ES cells successfully achieved delivery and functional expression of human immunoglobulin genes in mice, suggesting the potential of chromosome vector. Thus we are prompted to address the feasibility of constructing chromosome-based ex vivo gene delivery system.

We aimed at developing a structurally defined HAC into which circular DNA can be inserted by Cre/loxP system. The sequence defined human chromosome 21 was manipulated in chicken DT40 cells, i.e. truncation of distal q-and p-arm by telomere seeding and targeted introduction of a loxP site. These $21 \mathrm{HAC}$ vectors were successfully transferred and stably maintained in human HT1080 and mouse ES cells. The GFP gene inserted in the HAC expressed persistently in the HT1080 hybrids. These results indicated that the $21 \mathrm{HAC}$ vector might be useful for functional study of transgene in cells.

Progress in testing the performance of the HAC vector will be also reported; 1) hepatocyte specific expression of reporter gene under regulation of albumin promoter, 2) induction of tissue specific expression of reporter gene accompanying in vitro differentiation of human Mesenchymal Stem Cells and 3) construction of non-beta cells in which insulin expression is inducible. (Correspondence to oshimura@grape.med.tottori-u.ac.jp)

\section{PO:08:19}

\section{Construction of mammalian artificial} chromosome vectors for the expression of therapeutic genes by recombineering

G. Kotzamanis ${ }^{1}$, W. Cheung ${ }^{1}$, H. Abdulrazzak ${ }^{1}$, J. Gifford-garner ${ }^{1}$ and C. Huxley ${ }^{1}$

${ }^{1}$ Imperial College, London, UK
Mammalian Artificial Chromosomes (MACs) can be made by transfecting large $(>50 \mathrm{~kb})$ arrays of human centromeric (alphoid) DNA into human HT1080 cells in tissue culture. The transfected DNA forms stable, single-copy mini chromosomes de novo. These MACs constitute a potentially useful tool for gene therapy as intact genes with all their regulatory elements can be cloned into them and they should provide long term expression without the dangers of mutagenesis due to integration.

A method for adding large sequences onto BACs using homologous recombination based on the Red system has been developed. This method was used to add a 70-kb array of alphoid DNA (which has already been shown to be able to form de novo centromeres) to a $150-\mathrm{kb}$ BAC carrying the intact human HPRT gene. The potential usefulness of this BAC as a MAC vector for gene therapy of the Lesch-Nyhan syndrome was demonstrated by de novo formation of MACs expressing the HPRT gene in HT1080 cells.

MACs could also be useful vectors for gene therapy of cystic fibrosis. However, the intact CFTR gene (which spans 200-kb of genomic DNA) was not available in a single useful vector, though it was contained in three contiguous overlapping BACs which have been sequenced. A method for combining inserts of overlapping BACs using Red recombination has been developed. This method was used to clone the complete CFTR gene in one $\mathrm{BAC}$. This BAC is the basis for the construction of a MAC vector for CFTR expression.

\section{PO:08:20}

\section{Delivery of artificial chromosomes to human cells using HSV-1 amplicons}

D. Moralli ${ }^{1}$, K. Simpson ${ }^{1}$, R. Wade-Martins ${ }^{2}$, M. Assenberg ${ }^{1}$ and Z. Larin-Monaco ${ }^{1}$

${ }^{1}$ Weatherall Institute of Molecular Medicine, University of Oxford, John Radcliffe Hospital, Oxford, OX3 9DS, UK; ${ }^{2}$ The Wellcome Trust Centre for Human Genetics, University of Oxford, Roosevelt Drive, Oxford, OX3 7BN, UK

We are investigating an efficient method for delivery of HACs into human cells using a 
disabled Herpes Simplex Virus 1 system. The HSV-1 technology includes generating infectious HSV-1 amplicons in a helper virus free system where the HSV-1 genome lacks essential components for viral propagation. In this way BAC vectors are packaged into an HSV-1 capsid in a specific cell line (African Green Monkey cells), while HSV-1 viral particles themselves cannot be maintained or replicated. The amplicons which are generated can then transduce mammalian cells. We have prepared HSV-1 amplicons with HAC vectors containing alpha satellite DNA and infected several different human cell types including HT1080 (fibrosarcoma), G16-9 (glioma), MRC5V2 (lung fibroblast), 293 (kidney) and HaCAT (keratinocyte) cells. GFP expression was monitored after 24 hours, and neomycin resistant clones in HT1080, MRC5V2, 293 and G16-9 cells were isolated after 14 days following selection with G418. We determined that the efficiency of transient transfection of HAC vectors following HSV transduction is several orders of magnitude better than using lipofection as the delivery method. Positive clones have been selected and are being screened by FISH with specific alpha satellite probes for the presence of HACs and by immunofluorescence with CREST antisera for HACs with active centromeres.

\section{PO:08:21}

\section{Construction of episomal vectors carrying the intact human factor VIII gene for gene therapy}

\section{S. Perez-Luz ${ }^{1}$ and C. Huxley ${ }^{1}$}

${ }^{1}$ Division of Biomedical Sciences and MRC Clinical Sciences Centre, Imperial College School of Science Technology and Medicine, London, UK

Haemophilia A is a disorder caused by deficiency in the activity of factor VIII. Two aspects that make this disease attractive for gene therapy are that it is caused by mutations in just one gene and that low levels of expression are expected to have a therapeutic effect. So far, all gene therapy approaches have been based on expression of
FVIII from cDNA minigenes due to the large size of the gene $(180 \mathrm{~kb})$.

According to the Ensemble database, the BAC RP11-524G17, belonging to the RPCI human BAC library, contains the whole gene. However, after checking this BAC by restriction analysis and PCR we found that a $70-\mathrm{kb}$ NotI fragment is not present.

In order to make a BAC carrying the intact factor VIII gene, we have used homologous recombination (the Red system) in E. coli to recombine together two overlapping BACs, each containing part of the factor VIII gene. We now have a $230-\mathrm{kb}$ BAC containing not only all the exons of the gene, but also about $50 \mathrm{~kb}$ upstream of the first exon and $30 \mathrm{~kb}$ downstream of the last exon. Hopefully this BAC includes all the regulatory elements needed for full levels of tissue specific and controlled expression of the gene.

In a second step we have introduced onto this BAC either oriP/EBNA-1 which confers multicopy episomal maintenance, or alphoid DNA which is able to form mammalian artificial chromosomes (MACs). These constructs will be introduced into different human and mouse cell lines and expression of factor VIII will be analysed.

\section{PO:08:22}

\section{The behaviour of human $X$ centromere-based minichromosomes in human, mouse and chicken cell lines}

\section{J. Spence ${ }^{1}$, W. Mills ${ }^{1}$ and C. Farr ${ }^{1}$}

${ }^{1}$ Department of Genetics, University of Cambridge, Downing St, Cambridge, CB2 3EH, UK

A series of linear minichromosomes (ranging in size from several Mbs down to $450 \mathrm{~kb}$ ) has been generated through the targeted truncation of a human $\mathrm{X}$ chromosome maintained in chicken DT40 cells. In DT40 human minichromosomes as small as $1 \mathrm{Mb}$ are maintained very stably (loss rates/gen. $<0.5 \%$ ). The $450 \mathrm{~kb}$ chromosome has a loss rate of $\sim 4 \%$, but has immunofluorescence(IF)-detectable CENP-A and -C, indicating the presence of at least a partially functional centromere. In human HT1080 cells linear 
minichromosomes of $2-3 \mathrm{Mb}$ exhibit loss rates of $<0.1 \%$ and good copy number control. Structures below $1.8 \mathrm{Mb}$ show increasingly higher loss rates, with a $850 \mathrm{~kb}$ chromosome being lost at $\sim 3 \%$ per gen. (again CENP-C is detectable). In mouse LA-9 cells a $2.7 \mathrm{Mb}$ human minichromosome (with $>2 \mathrm{Mb}$ of DXZ1 alpha-satellite DNA) behaves acentrically: loss rate of 5-10\% per gen., no IF-detectable CENP-C and a hypervariable copy number on selection $(0->50 /$ cell $)$. Larger human chromosomes based on the same $\sim 3 \mathrm{Mb}$ DXZ1 array have been transferred into LA-9. A $\sim 140 \mathrm{Mb}$ human $\mathrm{X}$ chromosome and a $50 \mathrm{Mb}$ derivative are both very stable, bind CENP-C and show good copy number control. A $\sim 10 \mathrm{Mb}$ derivative, however, has low mitotic stability although it does show better copy number control than the $2.7 \mathrm{Mb}$ minichromosome, with CENP-C detectable on some chromosomes. The sharply differing mitotic behaviour of human $\mathrm{X}$ centromere-based chromosomes in chicken, human and mouse cells suggests that species differences in centromere function exist.

\section{Meiosis Symposium}

\section{L120}

\section{Factors influencing recombination levels in mammals}

\section{P. Hunt ${ }^{1}$}

${ }^{1}$ Department of Genetics and Center for Human Genetics, Case Western Reserve University and University Hospitals of Cleveland, Cleveland, Ohio 44106-4955

The production of haploid gametes requires unique modifications to the cell division process. The most complex is the establishment of physical associations between homologous chromosomes during prophase. Studies in yeast, flies and mammals have demonstrated that this physical tethering serves an essential function in facilitating homolog segregation at the first meiotic division. Moreover, recent studies in humans have demonstrated that not only the number of recombination events but their placement along the length of the chromosome influences the fidelity of chromosome segregation.
Recombination in female mammals occurs during prenatal development, thus, direct studies of recombination in humans have been largely limited to the male. To study the factors that influence the number and placement of exchange events in mammals, we have utilized a variety of mouse models. Naturally occurring and targeted gene mutations in mice are not only providing insight to the role of individual genes in the meiotic process but also contributing to our understanding of sex-specific differences. In addition, rodent studies also suggest that environmental exposures have the ability to influence meiotic recombination in both males and females. The combined results of these studies demonstrate the complexity of the meiotic process in mammals and raise a host of new and urgent questions. Importantly, they suggest that understanding and preserving human fertility will require the combined efforts of reproductive biologists, geneticists, and toxicologists.

\section{L121}

\section{How do cells hold sister chromatids together during mitosis and meiosis?}

\author{
K. Nasmyth ${ }^{1}$ \\ ${ }^{1}$ IMP (Research Institute of Molecular \\ Pathology), Dr. Bohr-Gasse 7, A-1030 Vienna, \\ Austria
}

There are two fundamental problems facing any cell trying to segregate sister DNA molecules. The first is how to disentangle them and package them into a confined space and the second is how to ensure that sister DNA molecules are placed in opposite sides of the cell. The former depends on the condensin complex whereas the latter relies on amphitelic attachment of sister kinetochores to microtubules with opposite orientations. Because sister DNA molecules produced by replication are held together by the cohesin complex, amphiletic attachment results in tension. Syntelic attachments, which do not result in tension, are selectively destabilized through the action of the Ipl1/Aurora B kinase. When and only when all chromatid pairs have come under tension is a cysteine protease called separase activated through the removal of its inhibitory chaperone, securin, by a ubiquitin protein ligase called the Anaphase-promoting complex. Separase triggers 
disjunction of sister molecules at anaphase onset by cleaving cohesin's Sccl subunit. Both cohesin and condensin contain SMC proteins, dimers of which form $\mathrm{V}$ shaped molecules with $50 \mathrm{~nm}$ long arms with ABC-like ATPases at each head. Cohesin's Scc1 subunit links its two SMC heads and thereby forms a gigantic ring structure with the potential for trapping DNA strands inside. Sccl belongs to a superfamily of related proteins (kleisins), some of which bind to SMC proteins in bacteria while others bind to SMC proteins in condensin.

\section{L122}

\section{SMC1b is required for mammalian chromosome dynamics, sister chromatid cohesion, and DNA recombination in meiosis}

\section{E. Revenkova ${ }^{1}$, M. Eijpe $^{2}$, C. Heyting ${ }^{2}$,} C. Hodges ${ }^{3}$, P. Hunt ${ }^{3}$, B. Liebe ${ }^{4}$, H. Scherthan ${ }^{4}$ and R. Jessberger ${ }^{1}$

${ }^{1}$ Center for Gene Therapy and Molecular Medicine, Mount Sinai School of Medicine, New York, NY 10029, USA;

${ }^{2}$ Molecular Genetics Group, Wageningen

University, 6703 BD Wageningen, The Netherlands; ${ }^{3}$ Department of Genetics, Case Western Reserve University, Cleveland, $\mathrm{OH}$ 44106; ${ }^{4}$ Max Planck Institute for Molecular Genetics, Berlin, Germany

Sister chromatid cohesion ensures faithful segregation of chromosomes in mitosis and meiosis. For meiosis it has been specifically adapted to facilitate both meiotic divisions. Meiosisspecific components of the cohesin complex, including the recently described vertebrate SMC1 isoform SMC1b, were suggested to be required for meiotic sister chromatid cohesion and DNA recombination. We generated SMC1bdeficient mice to assess the role off SMC1b. Mice of both sexes are sterile. Male meiosis is blocked in pachytene, female meiosis is highly error-prone but continues until metaphase II. Prophase axial elements (AEs) are dramatically shortened, chromatin extends further from the AEs, chromosome synapsis is incomplete, and sister chromatid cohesion in chromosome arms and at centromeres is prematurely lost.
In addition, crossover-associated recombination foci are absent or reduced, and meiosisspecific perinuclear telomere arrangements are impaired. Thus, SMC1b plays a key role in meiotic cohesion, assembly of AEs, synapsis, recombination, and chromosome movements.

\section{L123}

\section{Establishing a molecular time scale for meiosis in human fetal oocytes}

\section{P. Cohen ${ }^{1}$ and M. Lenzi ${ }^{1}$}

\author{
${ }^{1}$ Albert Einstein College of Medicine, \\ Dept. of Molecular Genetics, \\ 1300 Morris Park Avenue, Bronx, New York \\ 10461, U.S.A.
}

Successful meiosis is dependent on three related processes during prophase $\mathrm{I}$ : the pairing and synapsis of homologous chromosomes, formation of the synaptonemal complex, and the initiation and resolution of recombination between homologs. The molecular mechanisms underlying these events have been well studied in many organisms, aided by the recent advent of meiotic mutant phenotypes in mice. However, our knowledge of human meiosis, particularly females, remains limited because of the difficulties associated with collection of meiotic cells from human embryos. The importance of such studies is underscored by the fact that human female meiosis is particularly susceptible to non-disjunction, with $\sim 50 \%$ of conceptuses being chromosomally aneuploid as a result of errors in maternal meiosis I. Thus, the studies presented herein were aimed at establishing a molecular timeframe for recombination in human fetal oocytes obtained from patients undergoing pregnancy terminations between 14-24 weeks gestation.

Chromosome spreads were stained for key recombination proteins, including RAD51, RPA, and the mismatch repair proteins, MSH4, MSH5, MLH1 and MLH3. These latter two proteins are now accepted to be cytological markers for crossover events and, in mice, the number of MLH1/3 foci on meiotic chromosomes at prophase $\mathrm{I}$ is remarkably consistent, corresponding to the exact number of crossovers observed at 
metaphase I. However, we show here that there is much variability in MLH1/3 focus frequency in human fetal oocytes. These results suggest that the mechanisms of recombination are poorly regulated in human oocytes and might in part explain the high rate of aneuploidy observed in human oocytes.

\section{L124}

\section{Plasticity of histone methylation states during mammalian meiosis}

\section{A. Peters ${ }^{1}$}

${ }^{1}$ Novartis Research Foundation, Institute of Molecular Pathology, Vienna, Austria

No abstract was submitted for this talk.

\section{L125}

\section{Towards production of a new mouse model for Down syndrome}

Y. Kazuki ${ }^{1}$, M. Kimura ${ }^{2}$, Y. Kai ${ }^{1}$, S. Abe ${ }^{2}$, C. Okita ${ }^{2}$, Y. Shirayoshi ${ }^{1}$, T. Wakayama ${ }^{3}$, K. Hanaoka ${ }^{4}$, K. Tomizuka ${ }^{5}$ and

M. Oshimura ${ }^{2}$

${ }^{1}$ Department of Molecular and Cell Genetics, Graduate School of Medical Science, Tottori University, Japan; ${ }^{2}$ Department of Biomedical Science, Institute of Regenerative Medicine and Biofunction, Graduate School of Medical Science, Tottori University, Japan; ${ }^{3}$ Laboratory for Genome Reprogramming, RIKEN Center for Developmental Biology, Japan; ${ }^{4}$ Laboratory of Molecular Embryology, Department of Bioscience, Kitasato University School of Science, Japan; 5Pharmaceutical Research Laboratory, KIRIN Brewery Co., Ltd., Japan

Trisomy 21 is the most common live-born human aneuploidy. It results in a constellation of features known as Down syndrome (DS). To investigate the gene dosage effects of an extra copy of human chromosome 21 (Chr 21) on various DS phenotypes, we generated chimeric mice carrying an intact Chr 21 via microcell-mediated chromosome transfer (MMCT) into mouse embryonic stem (ES) cells. These chimeric mice showed a wide variety of phenotypic traits of DS in humans. Furthermore, the ES cells led to the disturbance of cardiogenesis and the elevated apoptosis in pre-mature neurons in vitro.

Two-dimensional electrophoresis revealed that the endogenous mouse mlc2a and PEBP proteins were remarkably downregulated in hearts of the chimeric mice. Further, we truncated the Chr 21 at ETS2 loci (Chr 21E) in homologous recombination proficient chicken DT40 cells and made chimeric mice containing the Chr 21E. Expression of PEBP and mlc2a was not repressed in Chr 21E chimeras, suggesting that gene(s) from ETS2 to the 21qter control expression of these proteins in DS.

However, each chimeric mouse has a different pattern of trisomic and euploid cells, complicating analysis. We are developing mice that have Chr 21 and defined fragments of it translocated to a mouse chromosome, using a combination of telomere-directed chromosome truncation in DT40 cells and Cre-loxP mediated chromosome translocation in ES cells. These mice are expected to allow stable germ-line transmission of the defined Chr 21, circumventing the inherent difficulties in analysis of chimeras. The current progress and lessons learned from the project will be introduced.

\section{L126}

\section{Recurrent trisomy 21: four cases in three generations}

\author{
J. Gair ${ }^{1}$, R. Rupps', L. Arbour', R. Jiang ${ }^{1}$, \\ H. Bruyere ${ }^{2}$ and W. Robinson ${ }^{1}$ \\ ${ }^{1}$ Department of Medical Genetics, University \\ of British Columbia, Canada; ${ }^{2}$ Department \\ of Pathology, University of British \\ Columbia,Vancouver BC, Canada
}

While gonadal mosaicism can lead to recurrence of trisomy 21 for a single couple, recurrence of free trisomy 21 in multiple members of a single pedigree has rarely been reported. We hereby present an unusual pedigree with four cases of Down syndrome (DS) born to four separate women related through three generations of one family. The mothers were aged 18,21, 29 and $\sim 30$ at the time of the births. G-banded karyotypes in two of the mothers of DS individuals were normal and karyotypes of all of the DS individuals showed 
free trisomy 21. Microsatellites spanning chromosome 21 were typed in this family to determine if any regions were shared in common among the mothers of the DS children. The centromeric and telomeric regions of chromosome 21 could be excluded from being shared, and of those tested, only the markers D21S215 and D21S258 (21q11.2) and D21S1440 (21q22.3) were potentially shared. Two members of the pedigree including one DS mother carried supernumerary alleles at markers 2503J9TG, D21S369 and D21S215, which span the region from $21 \mathrm{p}-$ 21q11.1. FISH studies with probes for the chromosome 13/21 centromere and 21q22 showed an enlarged signal at the centromere and a diminished signal at 21q22, compared to a control slide, on one of the chromosomes. The level of meiotic recombination on chromosome 21 was unusually high in this family as well. We present a model (currently being tested) whereby a cryptic inversion with breakpoints in 21q11.2 and 21q22.1 explains the unusual findings in this family.

\section{PO:08:23}

\section{Spermatocytes, spermatozoa and} embryos: FISH study of a Robertsonian translocation t(13;14)(q10;q10)

\author{
J. Blanco', Z. Sarrate ${ }^{1}$, E. Anton ${ }^{1}$, \\ M. Parriego ${ }^{1}$, J. Santalo', J. Egozcue ${ }^{1}$ \\ and $F$. Vidal ${ }^{1}$ \\ ${ }^{1}$ Unitat de Biologia Celular. Facultat de Ciencies. \\ Universitat Autonoma de Barcelona, Spain
}

As a consequence of the meiotic behavior of translocated chromosomes, Robertsonian heterozygous carriers produce high numbers of unbalanced sperm. An integral meiotic study, from spermatocytes to spermatozoa, could be useful to understand the segregation pattern of the reorganized chromosomes and to determine the risk of transmission to offspring. Moreover, in carrier couples, embryos resulting from IVF cycles can be analyzed through the application of PGD techniques.

We report the meiotic behavior of a Robertsonian translocation carrier $\mathrm{t}(13 ; 14)(\mathrm{q} 10 ; \mathrm{q} 10)$.
A semen sample was obtained and processed for meiotic studies. Prior to the application of M-FISH protocol (determination of metaphase I configuration and segregation), MI and MII spermatocytes images were captured and coordinates were noted. M-FISH was subsequently applied and analysis was performed using a Cytovision 2.7 system (Applied Imaging Corporation). FISH techniques were used to determine the incidence of normal/balanced sperm. Embryos obtained from two IVF-PGD cycles were also screened. Chromosome content and segregation patterns were inferred from FISH signal evaluation. Interchromosomal effects (ICE) over chromosomes 18, 21, 22, $\mathrm{X}$ and $\mathrm{Y}$ was evaluated (Multi-FISH; aneuploidy screening in sperm and embryos).

Chain configuration was the most frequent figure observed in MI spermatocytes (77.27\%).

Normal/balanced sperm and embryos were present in $86.48 \%$ and $61.54 \%$ of the analyzed cells respectively. Screening of aneuploidies in sperm revealed negative ICE.

Overall, data obtained resulted in an exhaustive cytogenetic evaluation of the patient addressed to an accurate reproductive counseling.

Acknowledgments: Project-SAF2003-04312 (Spain)

\section{PO:08:24}

\section{Differing roles of the Rad51 paralogues in mitotic and meiotic homologous recombination.}

\section{J. Bleuyard ${ }^{1}$, M. Gallego ${ }^{1}$ and C. White ${ }^{1}$}

${ }^{1}$ CNRS UMR6547, Université Blaise Pascal, 24, avenue des Landais, 63177 Aubiere, France

Since its identification as the eukaryotic homologue of the bacterial recombinase RecA, the Rad51 protein has been the object of considerable interest. Rad51 catalyses the strand exchange reaction, which is the motor of homologous recombination (HR) events. Rad51 is thus involved in a number of vital cellular processes including recombination, chromosome maintenance and meiosis.

In addition to Rad51, five paralogues of Rad51 have been shown to play roles in mitotic 
recombination, DNA repair and chromosome stability. These proteins are essential for embryonic viability in vertebrates and this fact has meant that understanding of their roles in meiosis is relatively limited. We have recently reported that the Arabidopsis homologue of one of these Rad51 paralogues (ATXRCC3) is involved in DNA repair and that its absence leads to a sterility phenotype due to extensive chromosome fragmentation during meiosis, first visible in diplotene of meiotic prophase (1). We have now identified Arabidopsis mutants of three other Rad51 paralogues (ATRAD51B, ATRAD51C and ATXRCC2). While disruption of any of the paralogues confers hypersensitivity to DNA damaging treatments, the atrad51c mutant is the only one to show dramatic meiosis defects. Our results point to differing roles for Rad51 paralogues in mitotic and meiotic HR. While the Rad51C/Xrcc3 complex is involved for both mitotic and meiotic $\mathrm{HR}$, the $\mathrm{Rad} 51 \mathrm{~B} / \mathrm{C} / \mathrm{D} / \mathrm{Xrcc} 2$ complex is only required for mitotic HR.

(1) Bleuyard et al (2004) EMBO J 23: 439-449

\section{PO:08:25}

\section{In situ analysis of radiation-induced DNA double-strand breaks and their repair by homologous recombination during murine male meiosis}

\author{
A. Chicheportiche ${ }^{1}$, J. Bernardino-Sgherri ${ }^{1}$ \\ and B. Dutrillaux ${ }^{1}$ \\ ${ }^{1}$ Unité Mixte de Gamétogenise et Génotoxicité, \\ INSERM U566-CEA-Paris VII, BP N N F-92260 \\ Fontenay-aux-roses, France
}

DNA double strand breaks (DSBs) are the major cytotoxic lesions induced by ionising radiation leading to chromosomal aberrations. During prophase I of meiosis, programmed DSBs are generated by the transesterase SPO11 to initiate homologous recombination involved in crossingovers. These events are required for normal haploid gamete production. Phosphorylated H2AX histone (gamma-H2AX) has been shown to point out DSBs and participate in their repair. During meiosis, it is associated with Spol1-dependent DSBs at leptonema and zygonema and is localised on the XY body at pachynema. In order to analyse the potential effect of radiationinduced DSBs during meiosis on meiotic recombination, we performed immunodetection of DSBs and crossovers on mouse spermatocyte chromosome spreads with gamma-H2AX and MLH1 antibodies, respectively. In non-irradiated pachynema, numerous small gamma-H2AX foci were observed along the pairing region of homologous chromosomes (synaptonemal complex SC). Gamma-radiation exposure at pachynema induced large gamma-H2AX foci assumed to decorate DNA loops attached to the SC. The number of MLH1 foci was unchanged, which suggests that these DSBs were repaired by gene conversion or alternative recombination pathways. By contrast, after a low dose of gamma-radiation given at preleptotene/leptotene stage, a significant elevated rate of gamma-H2AX foci was observed along the SC at early pachynema. An increased rate of MLH1 foci was also found at late pachynema and we propose that some radiationinduced DSBs were repaired by the homologous recombination pathway. All together, these results will permit to better understand the repair process of radiation-induced DSBs in meiosis.

\section{PO:08:26}

\section{Chromosome synapsis under the effect of colchicine in wheat}

\author{
E. Corredor ${ }^{1}$ and T. Naranjo ${ }^{1}$ \\ ${ }^{1}$ Departamento de Genética, Facultad de \\ Biologia, Universidad Complutense, 28040 \\ Madrid, Spain
}

Colchicine has been described as an agent able to affect chromosome pairing at metaphase $\mathrm{I}$ in bread wheat when applied at premeiotic interphase. With the aim of investigating the effect of colchicine on chromosome synapsis, immature spikes of wheat carrying two rye telosomes were injected with colchicine. The behaviour of the rye homologous chromosomes was studied using multiple FISH with telomere, a rye-specific centromere and rye genomic DNA probes performed on spread cells of anthers collected between two and four days after colchicine injection. In meiocytes of the control plants, chromosome synapsis 
starts at the bouquet stage, by both the telomeric and the centromeric chromosome ends, provided the centromeric end is incorporated to the telomeres cluster. Synapsis progress quickly and can be completed before the bouquet is disorganized. Meiocytes at early prophase I from anthers collected two days after colchicine application showed a similar behaviour to that of untreated material. However, meiocytes at prophase I of anthers collected three or four days after colchicine application showed a delay in the initiation and development of chromosome synapsis. In these cells, with rye chromosomes completely separated in some cases, the level of synapsis between rye chromosomes was lower than in the control material. Meiocytes affected by colchicine treatment showed a preference for the initiation of synapsis at the telomeric end of the rye telosomes. At diplotene-diakinesis, rye univalents were observed in all treated plants, prompting a possible effect of colchicine on chiasma formation in addition to that on the initiation of synapsis.

\section{PO:08:27}

\section{Association and migration of telomeres are two steps of the bouquet organization in hexaploid wheat}

\author{
E. Corredor ${ }^{1}$ and T. Naranjo ${ }^{1}$ \\ ${ }^{1}$ Departamento de Genética, Facultad de \\ Biologia, Universidad Complutense, 28040 \\ Madrid, Spain
}

Chromosomes adopt the bouquet configuration at the onset of meiosis in polyploid wheats. Concomitant to the congregation of telomeres, centromeres form complex multimeric structures in the opposite pole. Earlier works demonstrated the existence of a premeiotic interphase stage sensible to the colchicine action. Application of colchicine at this stage causes irregular chromosome pairing at metaphase I. Whether colchicine action is related to centromere association and chromosome movements produced during the bouquet formation has been studied in meiotic cells isolated from immature spikes injected with colchicine. Anthers at early meiotic stages were collected two or three days after colchicine treatment and the centromere and telomere position revealed by two colours fluorescence in situ hybridisation with centromere and telomere DNA probes. At premeiotic interphase the number of centromere signals is close to the haploid number. At the early bouquet stage a number of centromeres associate in multimeric structures but other remain associated in pairs or separated as deduced from the observation of both big and small fluorescence signals. Complex centromere clusters get resolved in centromere pairs before the bouquet is disorganized. Colchicine does not affect centromere association. By contrast, telomere grouping is severely affected by the colchicine action. Nuclei that started meiosis in the presence of colchicine show multiple telomere clusters scattered throughout the nuclear membrane in the hemisphere opposite the centromeres. Migration of dispersed telomere clusters to the telomere site is impeded by the colchicine action. This arrangement may represent an intermediate stage of the bouquet formation.

\section{PO:08:28}

\section{Cytogenetic processes of Secalotriticum genome formation}

\section{Gordei ${ }^{1}$ and $O$. Lyusikov ${ }^{1}$}

${ }^{1}$ Institute of Genetics and Cytology at National Academy of Sciences of Belarus

Investigations on development of secalotriticumrye-wheat amphidiploids with rye cytoplasm were carried out with a view to intensify rye genome expression for increasing ecological resistance of remote rye $\mathrm{x}$ wheat hybrids (Inventor's certificate 1734602).

Genome and chromosome composition of ryetriticale pentaploids of F1 (RRABR, 5/35) defines meiosis distinctions and is a key stage in secalotriticum genome formation. A high level of homeologous chromosome pairing of A- and Bgenomes was observed in hybrid diakinesis. It was related with inhibition of wheat $\mathrm{Ph}$-system with a triple dose of rye genes under conditions of rye cytoplasm (with 21 univalents expected, on the average $11,4 \mathrm{I}+6,3 \mathrm{II}$ ring $+4,4 \mathrm{II} \operatorname{rod}+0,6 \mathrm{III}+0,1$ were observed). Equational division of univalents 
turned out a basic source of meiosis anomalies. As a result, gametes varied from 14 to 28 in the chromosome number in the F1 hybrids. Classes $17-18(33,2 \%), 14(27,2 \%)$ and $21(24,8 \%)$ prevailed, unreduced gametes with 35 chromosomes did not occur. Gametes with 21 chromosomes (7R7A7B) balanced for genome composition were formed at partial meiotic unreduction with the frequency of $0,3-2,2 \%$. This process includes a normal division of rye bivalents, predominantly reduction division and uneven segregation of A-, B- and R-univalents, or equational division and meiotic elimination of R-univalents. Partially, unreduced gametes were the basis for producing secalotriticum according to the applied scheme already in the second generation (F1BC1 was identified by C-banding).

The developed secalotriticum is a genomebalanced stable haxaploid form with high values of agronomic traits.

\section{PO:08:29}

\section{Synatonemal complex length is the principal factor determining the difference in rate of recombination in male and female germ cells}

\author{
M. Hultén ${ }^{1}$ and C. Tease ${ }^{1}$ \\ ${ }^{1}$ Department of Biological Sciences, University \\ of Warwick, Coventry CV47AL, UK
}

Crossing-over at meiosis can be analysed using either genetic or cytogenetic methods (reviews in Hultén and Tease 2003, Nature EHG 2, pp 882887 and 876-881). The cytogenetic approach provides an overview of patterns of meiotic recombination that cannot be obtained in any other way. Spermatocytes have approximately 50 crossovers per cell in comparison to more than 70 in oocytes (corresponding to average genetic map lengths of 2500 and $3500 \mathrm{cM}$, respectively). Meiotic prophase I bivalents (Synatonemal Complexes, SCs) are considerably longer in human oocytes than spermatocytes. We have examined the influence of this factor on meiotic recombination in male and female human germ cells. Analyses of inter-crossover distances (and presumptively crossover interference) along SCs by the DNA mismatch repair protein MLH1 suggested that while there might be inter-individual variation there was no consistent difference between sexes. Thus the higher rate of recombination in human oocytes is not a consequence of more closely spaced crossovers along the chromosomes. We propose that SC length, and therefore the size of the physical platform for crossing-over, is the principal factor determining the difference in rate of recombination in male and female germ cells. One fundamental outstanding question is the background for the sex-specific architecture of meiotic pachytene chromosomes. This could be manifested, for example, by variations in the number and sizes of the chromatin loops associated with the axes of pachytene chromosomes. Our preliminary FISH observations indicate that SC loops may be considerably smaller in oocytes than in spermatocytes.

\section{PO:08:30}

\section{Chromosome linear elements and meiotic recombination hot spot activation in $S$. pombe}

R. McFarlane ${ }^{1}$, J. Loidl ${ }^{2}$, P. David ${ }^{1}$, W. Jenny ${ }^{1}$, S. Julia ${ }^{1}$, D. Emma ${ }^{1}$ and A. Lorenz ${ }^{2}$

${ }^{1}$ NWCRF Institute, School of Biological Sciences, University of Wales Bangor, Deiniol Road, Bangor, Gwynedd, LL57 2UW, UK;

${ }^{2}$ Department of Cell Biology and Genetics, Institute of Botany, University of Vienna, Rennweg 14, A-1030, Vienna, Austria.

The mechanisms regulating the function of recombination during meiosis are heavily influenced by the processes which control recombination initiation, including homologue pairing and activation of specific hot spot chromatin.

We have identified Rec10 as a central regulator/component of the proteinacious structures known as linear elements (LEs), which form in meiotic prophase and are implicated in interhomologue pairing. During meiotic prophase immunostaining using antibodies against Rec10 reveals that Rec10 is an integral part of LEs. Analysis of the amino acid sequence of Rec10 reveals 
a region of homology with the Red 1 protein of $S$. cerevisiae which is essential for the formation of structures analogous to LEs in this organism, indicating a highly conserved mechanism for chromatin regulation in meiosis $\mathrm{I}$.

We have identified a unique mutant allele of rec10, rec20-144. Analysis of recombination in this mutant demonstrates that there are differential context-dependent requirements for activation of the meiotic recombination hot spot M26. These data implicate Rec10 in the regulation of the localised chromatin dynamics during hot spot activation. Previous work has also shown a similar differential requirement for RED1 in hot spot activation in S. cerevisiae, providing further evidence of a highly conserved function for LE components. Given that the majority of meiotic recombination is likely to be initiated at hot spots, the study of the role of Rec10 and LEs in hot spot activation gives us an important insight into the mechanisms regulating proper chromosome segregation during meiosis.

\section{PO:08:31}

\section{Cytological analyses on a male sterile ramie (Boehmeria nivea Gaud.) and in some of its $F 1$ offspring}

\section{N. Pierozzi ${ }^{1}$, C. Pinto Maglio ${ }^{1}$, R. Baroni ${ }^{1}$} and R. Benatti $\mathrm{Jr}^{2}$

${ }^{1} \mathrm{CPD}$ Recursos Genéticos Vegetais, Instituto Agron Mico (IAC), Av. Baro de Itapura 1481, Campinas, SP, Brasil, CEP:13001-970; ${ }^{2}$ NPD Jardim Botanico, IAC, Brasil

Boehmeria nivea (ramie) is a perennial, wind pollinated, monoecious plant, native from Asia, with small diclinous flowers. It is known for its textile fibers. Cytological analyses were carried out on a male sterile diploid ramie plant, obtained after a previous treatment with colchicine solution for 72 hours in seed phase, and on a normal male fertile control plant and in some F1 descendents of this crossing. Young buds in different developing phases were collected, fixed in Carnoy and stored. Cytological preparations were done by squashing with acetic carmine. Some buds were selected for histological section cuts to know if there were some disturbances in the tapetal tissue. The analyses showed that microsporogenesis was normal in male fertile, but in male sterile, it was disturbed in the tetrad stage. At this time, a degenerative process was observed. The tetrad cell cytoplasm became wrinkled and with vacuoles. The tetrad callose was thicker in male sterile than in male-fertile plants. Section cuts showed that tapetal cells were slightly larger in male sterile than in male fertile. According to the observations, these disturbances did not involve structural chromosome changes, and chiasma mean value differences in metaphase I, observed among all the plants were not significant. The degenerative process also took place in young male buds; they became shrunken, full of aborted mature tetrads and fell down. It seems that this process is under nuclear control since half of the offspring inherited it. Male sterile may be a useful tool in breeding programs.

\section{PO:08:32}

\section{Rec8 distribution throughout meiotic prophase $I$ in euploid and aneuploid human oocytes}

\author{
P. Robles ${ }^{1}$, R. Garcia ${ }^{1}$, I. Roig ${ }^{1}$, J. Egozcue ${ }^{1}$ \\ and M. Garcia ${ }^{1}$
}

${ }^{1}$ Unitat de Biologia, Departament de Biologia Celular, Fisiologia i Immunologia, Facultat de Medicina, Universitat Autonoma de Barcelona, 08193 Bellaterra, Spain

Recent publications have shown the role of cohesin Rec8 in sister chromatid cohesion during mammalian meiosis. Most of the studies are based on male meiosis since the meiotic prophase I occurs during the fetal life period in female, so few samples are available.

We have studied the distribution of Rec 8 throughout meiotic prophase I in human oocytes applying inmunofluorescence against Rec8 and SCP1, which was used as control to elucidate the pairing process since it appears at synapsed regions.

Rec8 appeared at preleptotene, the signal was dispersed whereas at leptotene and zygotene was organized in a fibrillar pattern forming the axial 
elements of the synaptonemal complex. In addition in this stage, zygotene, colocalization of SCP1 and Rec8 was observed in the synapsed regions. At pachytene homologues pairing was completed and colocalization was observed along the whole length of the synapsed chromosomes. At diplotene, Rec8 staining was retained along the fibers.

For the first time, we also studied Rec8 pattern in aneuploid fetuses: $(47, \mathrm{XX}+21),(47, \mathrm{XX}+18)$ and $(45, \mathrm{XO}-47, \mathrm{XXX})$. Rec8 was always observed along the whole length of all the homologues. Alignment of the three homologues at pachytene in trisomic oocytes 18, 21 and $\mathrm{X}$ was observed originating a bivalent plus an univalent partially synapsed, a bivalent plus a completely unsynapsed univalent or a complete synaptic adjustment originating a trivalent.

In conclusion, Rec8 staining dynamics observed in euploid and aneuploid oocytes in this study are in agreement with previous studies published. Furthermore, existence of synaptic adjustment may delay meiotic prophase progression in aneuploid oocytes.

\section{PO:08:33}

\section{Homologue chromosomes pairing process analysis in $47, \mathrm{XX}+18$ human oocytes}

\section{Roig ${ }^{1}$, P. Robles ${ }^{1}$, R. Garcia ${ }^{1}$, J. Egozcue ${ }^{1}$, H. Schertan ${ }^{2}$ and M. Garcia ${ }^{1}$}

${ }^{1}$ Departament de Biologia Celular, Fisiologia i Immunologia.Universitat Autonoma de Barcelona, 08193-Bellaterra, Spain;

${ }^{2}$ Inst.Radiation Biology, BW, Neuherbergstr.11, 80937-München, Germany

Aneuploidy is one of the major causes of pregnancy loss in humans and it is mainly caused by chromosome non-disjunction during oogenesis. Little is known about mammalian female meiotic first stages because these ocurr during fetal life period. We performed an analysis of the homologues chromosomes pairing process occurring at meiotic prophase in a $47, \mathrm{XX}+18$ case and a euploid case by FISH.

At leptotene, most oocytes $(71.43 \%)$ presented three independent chromosome 18 signals.
During zygotene, pairing of the homologues chromosomes occurs, then oocytes having three signals $(21.67 \%)$, two $(36.67 \%)$ and one signal $(41.67 \%)$ were found. At pachytene, the vast majority of the oocytes $(91.92 \%)$ analysed presented one single signal, suggesting that pairing of the three homologues 18 was completed. Nevertheless some oocytes presented two separate signals, most likely corresponding to a bivalent plus a univalent. Diplotene oocytes displayed generally one signal $(83.33 \%)$. This results suggest that chromosome 18 pairing is an efficient process which ensures formation of a bivalent before pachytene in trisomic cases. In this sense, we commonly observed at zygotene that trivalent formation, or alignment of the three chromosomes 18 , was preceded by the existence of a bivalent plus an univalent.

Surprisingly, presence of an extra chromosome 18 had not detectable effects on other chromosomes pairing. Chromosome 13 pairing study in aneuploid and euploid cases gave non distinguishable results.

These findings supports the existence of a pairing checkpoint at human female pachytene and contradict the hypothesis of an interchromosomal effect seen in other studies.

\section{PO:08:34}

\section{Meiotic studies in OAT patients using M-FISH}

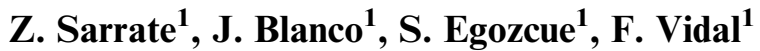 \\ and J. Egozcue ${ }^{1}$ \\ ${ }^{1}$ Unitat Biologia Celular, Universitat Autonoma \\ Barcelona, Bellaterra 08193, Barcelona, Spain
}

A method combining the analysis of classical meiotic preparations followed by M-FISH has been developed to assess the frequency of chiasmata and to determine if anomalies affect specific bivalents in testicular material.

Samples were obtained by unilateral biopsy from 8 OAT patients and processed for meiotic studies. Prior to the application of M-FISH protocol, slides were stained with Leishman. MI and II spermatocyte images were captured, coordinates were noted and chiasma frequency was 
determined for every single bivalent. M-FISH procedures were subsequently applied (Spectra Vysion Assay, Vysis Inc) and analysis was performed using a Cytovision 2.7 system (Applied Imaging Corporation).

Specific results for every single bivalent (rang, mean and standard deviation) in all cells studied were obtained through the analysis of 113 MI spermatocytes. Overall, a slight reduction in the number of autosomal chiasma frequency was detected (47.8) compared to controls (51.3; Laurie and Hultén, 1985). Sex bivalent was observed in $83.3 \%$ of the MI analyzed. Synaptic anomalies affecting the sex chromosomes (structural reorganization), 9 (break) and 22 (asynapsis) were observed in three different cells.

43 MII cells were analyzed. Chromosome anomalies were detected for chromosome 2 (2.6\%; abnormal morphology), 3 (5.1\%; break), 6 (2.4\%; break), 9 (7.3\%; break) and gonosomes ( $2.6 \%$; numerical anomaly).

Results confirm the high presence of synaptic errors resulting in a reduction in the number of chiasmata in OAT patients. Non-disjunctional errors or small reorganizations, overlooked in classic meiotic preparations, were identified. Preliminary results seem to indicate that gonosomes and chromosome 9 are more affected by synaptic anomalies than others.

Acknowledgments: Project-SAF2003-04312 (Spain)

\section{PO:08:35}

\section{Silencing of unpaired meiotic DNA in the mouse}

\section{J. Turner ${ }^{1}$, S. Mahadevaiah ${ }^{1}$, C. Deng ${ }^{2}$ and $P$. Burgoyne ${ }^{1}$}

${ }^{1}$ MRC National Institute for Medical Research, London, UK; ${ }^{2}$ National Institutes of Health, Bethesda, Maryland, USA

In Neurospora, DNA unpaired in meiosis is both silenced and induces silencing of all DNA homologous to it, including genes that are themselves paired. This mechanism has been called Meiotic Silencing by Unpaired DNA (MSUD) and is thought to protect the host genome from invasion by transposable elements and viruses. Silencing of unpaired segments during meiosis has also been documented in Caenorhabditis elegans; in this case silencing of the single $\mathrm{X}$ chromosome during XO male meiosis is critical for imprinted $\mathrm{X}$ inactivation in $\mathrm{XX}$ offspring, and is abolished when the single $\mathrm{X}$ chromosome is provided with a pairing partner (XX, sex reversed males). We now demonstrate that silencing of unpaired meiotic DNA also takes place in the mouse. In mouse mutants harbouring errors in meiotic pairing (synapsis) such as XO females, males carrying the $\mathrm{X}$-autosome translocation $\mathrm{T}(\mathrm{X} ; 16) 16 \mathrm{H}$ and Msh5 null males, unpaired chromosome segments are transcriptionally silenced, as revealed by a recently developed Cot-1 RNA FISH technique that identifies nascent nuclear transcripts. This silencing mechanism is dependent upon the tumour suppressor gene Brcal, which has previously been implicated in two related process; Somatic X Chromosome Inactivation and Meiotic Sex Chromosome Inactivation (MSCI). Silencing of unpaired DNA during mouse meiosis provides novel insights into the relationship between asynapsis and sterility in mammals and a broader understanding of the biology of Brca1.

\section{PO:08:36}

\section{Human artificial chromosomes as tools for transgenesis and for analysing mammalian meiosis.}

T. Voet ${ }^{1}$, J. Vermeesch ${ }^{1}$, E. Schoenmakers ${ }^{2}$, B. Liebe ${ }^{3}$, S. Carpentier ${ }^{4}$, C. Labaere ${ }^{1}$, J. Verbeeck ${ }^{1}$, H. Scherthan ${ }^{3}$ and P. Marynen ${ }^{1}$

${ }^{1}$ Department of Human Genetics, University of Leuven, Belgium; ${ }^{2}$ Department of Medicine, University of Cambridge, UK;

${ }^{3}$ Max-Planck-Institute for Molecular Genetics, Berlin, Germany; ${ }^{4}$ Laboratory of tropical plant sciences, University of Leuven, Belgium

We have generated a circular chromosomal vector (CV) consisting of human alphoid 20 and 1p21-22 sequences, while detectable telomeres are lacking. In addition to transgenic applications, such artificial chromosomes represent a powerful 
technology for studying meiotic chromosome behaviour and checkpoints.

Proof-of-principle was provided for both: (1) the $\mathrm{CV}$ is mitotically stable in different mammalian cell lines, (2) the $\mathrm{CV}$ is transferrable between cell lines by microcell-mediated chromosome transfer, (3) plasmids and PACs can be inserted by the Cre/loxP system without disturbing its relevant properties, (4) mouse ES-cells carrying the $\mathrm{CV}$ with or without insert can be used to generate trans-chromosomal mice, (5) genes present on the $\mathrm{CV}$ are expressed in a tissue-specific manner at levels compatible with the hemizygous presence of the transgene and (6) male and female mice, either monosomic or disomic for the $\mathrm{CV}$, show germline transmission of the CV. Detailed spermatogenic analysis revealed a telomere-independent mechanism for homologous $\mathrm{CV}$ pairing and illuminate escape routes to meiotic checkpoints.

Recently, linearisation of the circular CV with the CRE/loxP system was performed. Potential linear CVs were detected by telomeric PNA-FISH and are currently being characterised in cell lines. Comparing the mouse meiotic data of the circular $\mathrm{CV}$ with this of a telomere-bearing version will further clarify the relevance of functional telomeres on chromosomes in mammalian bouquet formation, in bouquet-facilitated homologue pairing and in meiotic checkpoint bypass. Additionally, a linear CV will benefit from improved mitotic stability as it will eliminate CRE/loxPand sister chromatid exchange (SCE)-mediated duplications.

\section{Population Cytogenetics Symposium}

\section{L127}

\section{Population cytogenetics: the study of chromosome variation in natural populations}

\section{J. Searle ${ }^{1}$}

${ }^{1}$ Department of Biology, University of York, PO Box 373, York YO10 5YW, UK

Chromosomes have drifted in and out of favour as subject material for population geneticists.
A balanced view is that the study of chromosomes should form a small but important part of population genetics. Chromosomal variants are easily scored characters that can, for instance, be valuable as makers in phylogeography and for inferring population structure. They can also reveal population genetic principles such as the selection pressures involved in meiotic drive and in the maintenance of hybrid zones. However, chromosomal variants also have a role as evolutionary agents in their own right. They may, for example, be involved in maintaining high genic variation and in speciation. This talk will run through these various aspects of population cytogenetics as a prelude for the detailed case studies that follow.

\section{L128}

\section{On the role of chromosomal rearrangements in speciation}

\author{
K. Livingstone ${ }^{1}$ and L. Rieseberg ${ }^{2}$ \\ ${ }^{1}$ Department of Biology, Trinity University, San \\ Antonio, TX, USA; ${ }^{2}$ Department of Biology, \\ Indiana University, Bloomington, IN, USA
}

Recent data from a wide range of organisms and new theoretical developments have again suggested that chromosomal rearrangements may have a causative role in the speciation process. These developments will be reviewed along with new data from the Solanaceae in support of this hypothesis.

\section{L129}

How much is temperature responsible for latitudinal chromosomal clines in Drosophila? An experimental approach

\author{
A. Fontdevila ${ }^{1}$, M. Santos ${ }^{1}$, L. Serra ${ }^{2}$, \\ J. Balanya ${ }^{2}$ and $W$. Céspedes ${ }^{1}$ \\ ${ }^{1}$ Departament de Genetica i Microbiologia, \\ Universitat Autonoma de Barcelona; \\ ${ }^{2}$ Departament de Genetica, Universitat de \\ Barcelona, Spain
}

Chromosomal polymorphism in Drosophila subobscura shows concordant latitudinal clines 
between old Palaearctic original populations and recent colonizing populations of the Americas. This parallel observation in two hemispheres has been taken as the most convincing evidence that natural selection is at work. Yet, the environmental causes of these chromosomal gradients are largely unknown, being temperature the most favoured selective agent. In order to disentangle temperature effects, a controlled experiment using laboratory populations under different thermal regimes, in which other putative selective agents (namely, larval density) have been kept constant across temperatures, has been carried out. Results show a quick and consistent shift in gene arrangement frequencies in response to thermal selection regime, qualifying temperature as an effective selective agent. However, the analysis of arrangement frequency changes unveils a complex pattern of response to temperature often not consistent with the observed trends in natural clines. For example, Est inversion is the most responsive in both natural and experimental populations, yet the trend observed in the latter is the opposite of that predicted from latitudinal clines. Interestingly, a decrease of chromosomal diversity is observed at the warmest (sub-optimal) temperature, which is consistent with a similar diversity change in long-term studies related to climate warming in natural $D$. subobscura populations. In sum, our results indicate that temperature is an important selective agent but does not explain by itself the observed trends in chromosomal latitudinal clines.

\section{L130}

Locating long-term glacial refugia in the Mediterranean Basin.

\section{Autopolyploid complexes in Asplenium} as a model system

J. Vogel', S. Russell', M. Grundmann', F. Rumsey ${ }^{1}$, H. Hunt ${ }^{1}$, H. Schneider ${ }^{2}$, I. Pinter ${ }^{3}$, M. Gibby ${ }^{4}$ and J. Barrett ${ }^{5}$

${ }^{1}$ The Natural History Museum, London, UK; ${ }^{2}$ Universitaet Goettingen, Germany; ${ }^{3}$ University of Budapest, Hungary; ${ }^{4}$ Royal Botanic Garden, Edinburgh, UK; ${ }^{5}$ University of Cambridge, UK
In order to support conservation efforts and to identify long-term glacial refugia in Europe we are working with the fern genus Asplenium as a model system to investigate patterns and processes in polyploid plant evolution in the Mediterranean Basin. Long-term glacial refugia are areas where plants (and animals) have survived successive glaciation cycles, i.e. warm and cold stages during the Pleistocene. In order to find such areas we have explored the discontinuities in ploidy levels, distribution patterns, breeding systems and genetic diversity in several auto- and (segmental-) allopolyploid complexes in the fern genus Asplenium, combining field work and extensive laboratory studies (allozyme electrophoresis and cpDNA sequencing). Patterns observed in three autopolyploid complexes, A. ceterach, A. petrarchae and $A$. trichomanes, give us interesting insights into the dynamic polyploid evolution in the Pleistocene and allow us to infer the locations of long-term Pleistocene refugia in the Mediterranean Basin.

\section{L131}

\section{Episodic patterns of Robertsonian fusion; causes and consequences}

\section{Rowell ${ }^{1}$}

\author{
${ }^{1}$ School of Botany and Zoology, Australian \\ National University, Canberra, Australia
}

Some groups of animals experience repeated Robertsonian rearrangements while their karyotypically similar relatives show marked stability. Even within these, the pattern of rearrangement varies dramatically, with some groups showing rapid and repeated fusion across the whole karyotype ('catastrophic pan-fusion'), while others are characterized by the sporadic occurrence of individual fusions followed by fixation or extinction. Fissions are considerably rarer and do not appear to follow the same patterns. The mechanism(s) by which fusion occurs has not been elucidated, although a hereditary predisposition to fusion in one species points toward the involvement of a p-like transposable element. This presentation explores characteristics of 
fusion events and their potential adaptive significance.

\section{L132}

\section{Identification, variation and evolution of common fragile sites}

\section{Greenbaum ${ }^{1}$ and P. Dahm ${ }^{2}$}

${ }^{1}$ Department of Biology, Texas A\&M University, College Station, TX, 77843 USA; ${ }^{2}$ Department of Statistics, Texas A\&M University, College Station, TX 77843 USA

Although well studied in relation to human cancer, the genetics, variation and evolution of the so-called common fragile sites (CFS) remain undetermined. As molecular analyses have failed to identify any associated signature sequence, studies of CFS continue to depend on analyses of the distribution of chromosomal breaks/gaps. To investigate the basic genetics and evolution of CFS, we have adopted the null hypothesis that the per-individual presence or absence of any particular CFS reflects genetic variability among individuals and approximates a codominant allelic model. As this approach is dependent on the accurate per-individual identification of CFS, we (Bähm et al. Hum Genet 95:249, 1995) developed a multinomial statistical model (FSM) for the identification of CFS as chromosomal bands that express significantly nonrandom breakage relative to the distribution of total breakage in data from single individuals. FSM analyses were used to obtain initial estimates of presence/absence variation of aphidicolin-induced CFS among deer mice (Peromyscus maniculatus) and humans. In both cases, the analyses indicated that most CFS are polymorphic; the number of CFS per individual ranged from 720 with approximately $75 \%$ of the CFS occurring at frequencies less than 0.5 and only about $5 \%$ being fixed. Consistent with the hypothesis that CFS variation is allelic and polymorphic, cladistic analysis of preliminary CFS characterizations for four species of Peromyscus yielded evolutionary relationships entirely concordant with the corroborated phylogeny of these species.

\section{L133}

\section{ECARUCA: an internet-based European database for collection of rare chromosomal abnormalities}

\author{
I. Feenstra ${ }^{1}$, D. Koolen ${ }^{1}$, A. Siezen ${ }^{1}$, C. Evans ${ }^{2}$, \\ J. Fang ${ }^{2}$, R. Winter ${ }^{3}$, A. Schinzel ${ }^{4}$, M. Lees ${ }^{3}$, \\ B. De Vries ${ }^{1}$ and C. Van Ravenswaaij ${ }^{1}$ \\ ${ }^{1}$ Dept Human Genetics, Nijmegen, The \\ Netherlands; ${ }^{2}$ School of Computing Science, \\ Middlesex University, London, UK; ${ }^{3}$ Institute of \\ Child Health, London, UK; ${ }^{4}$ Institute of Medical \\ Genetics, Zurich, Switzerland
}

During recent years a considerable improvement in diagnostic techniques has enabled cytogeneticists to find more and smaller chromosomal aberrations. However, accurate clinical knowledge about rare chromosome disorders is frequently lacking, mostly due to a significant decline in published cases. On the other hand, there is an increasing demand from parents and physicians for reliable information about the disorder of their child or patient.

Therefore, we established the European Cytogeneticists Association Register of Unbalanced Chromosome Aberrations; http://www.ecaruca. net/.

This internet-based database, funded by the European Union, collects cytogenetic and clinical data of (non-) published cases with a rare chromosome abnormality. This can be microscopic visual aberrations, or micro-deletions and-duplications.

Genetic centres can submit data to the register by using the downloadable forms from the website, although online-submission will be possible as from June 2004. At present, ECARUCA contains around 4000 cases.

The availability of collected data is subdivided in a two-level system: each internet user can view general information about specific chromosome aberrations and the accompanying clinical features. Whereas, account holders can receive specific data per case, including additional research and clinical pictures.

The collection and exchange of clinical and technical knowledge will allow for accurate information on clinical aspects of rare chromosome disorders that can be used by professionals 
involved. Moreover, correlation study of chromosome aberrations and their phenotypes will be invaluable for localizing genes involved with mental retardation and congenital anomalies.

\section{L134}

\section{Determination of risk factors in carriers of peri- and paracentric inversions}

\author{
C. Pagenstecher ${ }^{1}$, B. Mub ${ }^{1}$ and G. Schwanitz ${ }^{1}$ \\ ${ }^{1}$ Institute of Human Genetics, University Hospital \\ Bonn, Germany
}

In 41,000 patients chromosome investigations were performed because of suspicion of chromosomal aberrations. We found a frequency of inversions of 2.2 per mill (91 patients). 90 patients had one inversion, one patient two. In 55 cases the inversion was pericentric, in 37 paracentric. Familial cases were predominant: there were $92 \%$ familial versus $8 \%$ de novo inversions. The three most frequent reasons for chromosome analysis were prenatal diagnosis because of advanced maternal age $(38.5 \%$, accidental finding), a child with malformations (17.5\%) and increased abortions (12.0\%). Analyses of inversions were done by conventional cytogenetics in 87 and by FISH in 4 cases. There was aneusomy by unequal recombination which will be illustrated in detail. The three most frequent inversions were those in chromosome 2, 10 and Y. Our results will be compared to findings in the literature.

\section{L135}

\section{Chromosome mutations in acrocentric chromosomes: frequencies and genetic relevance}

\section{G. Schwanitz ${ }^{1}$, L. Kalz ${ }^{1}$, A. Jelska ${ }^{2}$ and} A. Midro ${ }^{2}$

${ }^{1}$ Institute of Human Genetics, University Hospital Bonn, Germany; ${ }^{2}$ Department of Clinical Genetics, Medical University Bialystok, Poland

First, the different DNA structure of the short arm regions of the acrocentrics is given. Changes in these segments are then summarized. Polymorphisms in the constitutive heterochromatin are delineated for 600 persons of Central European origin and then compared to 2 groups of different ethnic origin. Then, from the own investigation group, structural rearrangements with breakpoints in eu- and heterochromatic bands are analyzed as to their frequency and aberration type. One subgroup of precisely defined chromosome aberration after QFQ-staining, namely Robertsonian chromosomal translocation (RobCT), were studied with the aim to estimate the frequency of the unbalanced progeny in case of inherited form of these RobCT. Genetic risk estimation has been performed according to the method of Stengel-Rutkowski and Stene. Risk values for maternal and paternal carriers have been obtained.

\section{L136}

\section{Population-genetic consequences of the Chernobyl accident in different mammalian species}

\author{
V. Glazko ${ }^{1}$ and T. Glazko ${ }^{1}$ \\ ${ }^{1}$ Research Institute of Biology, Rostov State \\ University, Rostov-on-Don, 344090 Russia
}

The increases of cytogenetic anomalies in the bone marrow cells of mice lines $\mathrm{BALB} / \mathrm{c}$, $\mathrm{C} 57 \mathrm{BL} / 6 \mathrm{j}, \mathrm{CC} 57 \mathrm{~W} / \mathrm{Mv}$, subjected to ionizing radiation (near 0.6 Sv) were revealed. However, only those types of cytogenetic anomalies were increased which were spontaneously highly variable in an age- or season-dependent manner in the same mice lines not subjected to radiation. In other experiments, three species of voles (Microtus arvalis, Microtus oeconomus, Clethrionomys glareolus), trapping in zone of alienation of Chernobyl NPP with different levels of ionizing irradiation $\left(<5 ; \sim 200 ; \sim 1000 \mathrm{Ci} / \mathrm{km}^{2}\right)$ were investigated. Among them, the evolutionary youngest species of common vole (Microtus arvalis) characterized by comparative high karyotype instability in area was the most sensitive to ionizing radiation. This interspecies comparison thus confirmed that an increase of ionizing radiation does not induce new genetic damages, but 
destabilizes the preexisting genomic ãhot spotsä that are either species-specific (and more characteristic to evolutionary young species) or genotype-specific (different laboratory lines of mice). In generations of cattle, born in experimental farm $\left(\sim 200 \mathrm{Ci} / \mathrm{km}^{2}\right)$ near Chernobyl NPP, disturbance of equiprobable transmission of alleles in a number of molecular genetic markers, increase of heterozygosity and radio resistance (on decrease of cytogenetic anomaly frequencies in blood cells in generations) were observed. The result of selection for irradiation resistance in voles emerged through approximately 26 generations after the beginning of the ionizing radiation exposure and it was dose-dependent. No increase in the quantity of constitutive mutations in investigated genes, ISSR-PCR markers or chromosomes in cattle and Rodentia species was observed.

\section{L137}

\section{Co-segregation of two sex linked translocation chains in the Australian social huntsman spider Delena cancerides.}

\author{
H. Sharp ${ }^{1}$ and D. Rowell ${ }^{1}$ \\ ${ }^{1}$ Department of Botany and Zoology, The \\ Australian National University Canberra, \\ 0200, Australia
}

Delena cancerides incorporates five well characterised chromosomal races. The ancestral form has a completely telocentric karyotype, while the other races are saturated for different combinations of Robertsonian fusions. Three of these races form chains of chromosomes at male meiosis due to fixed fusion heterozygosity. One race has a chain of three chromosomes, another a chain of five, and the most spectacular, a chain of nine chromosomes. These chains include a sex chromosome, and segregate as a complex XY system. This ensures that karyotypic integrity is maintained over successive generations, and the races remain stable.

We have recently found a new, distinct race of D. cancerides. At male meiosis this race forms two chains of five chromosomes. The presence of $\mathrm{X}$ chromosomes on both chains ensures a stable inheritance pattern, although coordination of the two chains is essential. This is the first stable, double chain system found in any organism, and the coordination of segregation patterns between two distinct translocation chains is unprecedented. Analysis of this chromosomal race will permit the investigation of the origins of fusion heterozygosity in $D$. cancerides, and may shed light on basic principles of meiosis such as the mechanisms behind chromosomal segregation, and sex chromosome evolution.

\section{PO:08:37}

\section{Translocations of chromosome 13 in the course of family investigation}

\section{G. Bujdosó ${ }^{1}$, P. Sótonyi ${ }^{1}$, O. Bellovits ${ }^{1}$, E. Csonka ${ }^{2}$ and G. Hadlaczky ${ }^{2}$}

${ }^{1}$ Hungarian Academy of Sciences, Semmelweis University, Institute of Forensic Medicine, Budapest, Hungary; ${ }^{2}$ Institute of Genetics, Biological Research Center, Hungarian Academy of Sciences, Szeged, Hungary

During determination of origin studies certain deviations and diseases surface in individuals coming from the normal population.

The first case is one from 30 years ago. The putative father did not accept his paternity of the eight years old boy, despite the blood group and anthropology results showing the possibility of his fatherhood. During the office visit of father and child for evaluation the intelligence of both was above average and they showed peculiar psychological behaviour. Based on these facts, this was the first time - even internationally - that chromosome studies were performed on all three parties (mother, child, defendant).

Karyotyping results included a normal in the mother and 46XY t $(2 q-; 13 q+)$ both in the child and the putative father. The paternity was proven.

Meanwhile the boy grew up and after thirty years he returned to our laboratory with his two children, owing to some serious behavioural problems with them. According to our chromosome investigation the daughter showed the same balanced translocation that her father and grand- 
father had. No translocation was found in the boy, but further genetic test results are pending.

In our other case a girl born at term (with $1600 \mathrm{~g}, 45 \mathrm{~cm}$ ) was searching for her genetic father. The chromosome studies resulted in the child and probable father having translocation of chromosomes $13 ; 14$.

At the time of the studies the girl was a dainty 21 months old, much smaller than her companions, but well proportioned and healthy, both physically and mentally.

\section{PO:08:38}

\section{Genetic diversity of Fagaceae from Chiangmai province, northern Thailand, based on 18S.26S ribosomal genes}

P. Chokchaichamnankit ${ }^{1}$, W. Chulalaksanakul ${ }^{1}$ and K. Anamthawat-Jonsson ${ }^{2}$

${ }^{1}$ Botany Department, Faculty of Sciences, Chulalongkorn University, Phayathai Road, Bangkok 10330, Thailand; ${ }^{2}$ Biology Department, University of Iceland, Askja-The Natural Science Building, Sturlugata 7, Reykjavik, IS-101, Iceland

The oak family (Fagaceae) in Thailand consists mainly of three genera: Castanopsis, Lithocarpus and Quercus. They are most common in northern Thailand, and the species diversity is impressive, i.e. 40 ö 60 species have been estimated from this region. Their habitats are relatively diverse, from hill evergreen forests down to dry dipterocarp forests and mixed deciduous forests with open grassland. We have selected areas of study that include all three forest types in Chiangmai province. At each site, trees were identified at regular intervals between them, regardless of species identity (based on local names, Fagaceae flora of Thailand is not yet complete), marked for further visits. Various sample types were collected from each plant, for herbarium and morphological assessment, for chromosome and DNA isolation. About 100 trees are included in this study and they are likely to represent more than 20 species. Total genomic DNA has been isolated from young leaves, where as mitotic chromosomes have been isolated from leaf buds and meiotic chromosomes from flower buds. Chromosome counting is performed on fluorochrome staining of leaf chromosomes. The $18 \mathrm{~S} .26 \mathrm{~S}$ ribosomal genes are mapped by fluorescence in situ hybridization (FISH) on both mitotic and meiotic chromosomes using the wheat pTa71 ribosomal DNA as probe. Genomic DNA from all samples are analysed for restriction fragment length polymorphisim (RFLP) using the same rDNA clone. The results will be presented. No such work on genetic diversity of this family in Thailand or Southeast Asia has been reported before.

\section{PO:08:39}

\section{'Ages' of chromosome translocations $t(15 ; 17), t(8 ; 21)$, and inv(16) in human acute myeloid leukaemia (AML).}

M. Colovic ${ }^{1}$, D. Vukomanovic ${ }^{2}$, M. Andolina ${ }^{3}$ and G. Jankovic ${ }^{1}$

${ }^{1}$ Institute of Haematology, Belgrade, Serbia and Montenegro; ${ }^{2}$ Mathematical Institute, Belgrade, Serbia and Montenegro; ${ }^{3}$ Istituto per L'Infancia, Trieste, Italy

Patients with AML may(+) or may not(-) carry chromosome translocations (CT) associated with underlying oncogene fusions. Leukaemogenic mutations do not accumulate with time but are purged in each generation. Nevertheless, proportions of their respective CT polymorphisms $(+/-)$ may have changed, but not remained frozen, during evolution. Initial proportion [Ro] of the CT (-) variant of leukaemogenic allele fusions ought to had been virtually 1.0 at some point back in time. Do present proportions of the $(-)$ polymorphism in leukaemia mark time carrying implications of a historical chronometer in dating evolutionarilly neutral CT? If the propensity for recurrent CT-generation in leukaemia patients follows the evolutionary dynamics of repetitive elements underlying this propensity, then we could age-date specific CT. Contemporary proportion $[\mathrm{R}]$ of the $\mathrm{CT}(-)$ variant of oncogene fusions characterizing three distinct subtypes of AML, and their incidence rates [substituted in place of a specific decay constant, (lambda)] were entered in a mathematical expression $[t=1 \quad 1 n$ 
Ro/R] that relates exponential decay and the time[t] elapsed since the CT first appeared. The age estimates obtained are 230,170 t(15;17), $182,958 \mathrm{t}(8 ; 21)$, and 163,248 (inv16) years. Present proportions of the $(-)$ polymorphisms [R] may represent enduring palaeocytogenetic trace of archaic tumour karyotypes. The age estimates of morbid CT concur with the emergence of first modern humans 250,000 to 150,000 years ago.

\section{PO:08:40}

\section{A CGH and FISH detection of a de novo 22q13-qter trisomy and 2pter monosomy in a patient: clinical presentation and review of the literature}

C. Hans' ${ }^{1}$, Y. Sznajer ${ }^{2}$, E. Vamos ${ }^{2}$, B. Pichon ${ }^{1}$ and L. Duprez ${ }^{1}$

${ }^{1}$ Laboratoire de Ctogénétique, Hopital Erasme, Université Libre de Bruxelles, Belgique; ${ }^{2}$ Hopital Universitaire des Enfants Reine Fabiola,

Université Libre de Bruxelles, Belgique

The phenotype of distal $22 \mathrm{q}$ trisomy is characterised by intra-uterine growth retardation, hypotonia, mental retardation, microcephaly and feeding difficulties. Hypertelorism, narrow palpebral fissures, small nose with anteverted nares, a thin upper lip, cleft lip/palate, low set and dysplastic ears delineate the dysmorphic features. Hypogenitalism is an additional finding in males (cryptorchidism, small penis, hypospadias).

Most of the affected patients reported to date die shortly after birth or have a short lifespan. The phenotypic picture of distal $2 p$ monosomy associates post natal growth retardation, microcephaly, bulging metopic suture, low set ears, flat nasal bridge, micrognathia, high arched palate, single palmar crease, foot deformities and developmental delay. We report clinical and cytogenetic findings in a 19-year-old boy with distal $22 \mathrm{q}$ trisomy and distal $2 p$ monosomy: the features displayed by our patient are rather related to $22 \mathrm{q}$ trisomy than $2 \mathrm{p}$ monosomy. Clinical comparison is established with the published cases. Our patient is the first report of partial 22 trisomy with such a long survival. Comparative Genomic Hybridisation $(\mathrm{CGH})$ has allowed to delineate the cytogenetics anomaly.

\section{PO:08:41}

\section{Human chromosomal Q-heterochromatin regions in individuals of various age}

\author{
A. Ibraimov ${ }^{1}$ \\ ${ }^{1}$ National Center of Cardiology and Internal \\ Medicine, Togolok Moldo str. 3, Bishkek, \\ Kyrgyzstan, 720040, C.I.S.
}

The quantitative content of chromosomal Q-heterochromatin regions (Q-HRs) was studied in individuals of different age groups taking into account their racial and ethnic affiliation. It was shown that chromosomal Q-HRs are most numerous in the genome of neonates, while they are the least numerous in the genome of elderly subjects (aged 60 years and older) regardless of the ethnic features of the individuals studied. Our data indicate that 1) in a population there is a clear-cut tendency towards a decrease in the number of chromosomal Q-HRs with age, regardless of racial and ethnic features on the individuals; 2) of all the age groups the genome of neonates contains the greatest number of $\mathrm{Q}$ HRs; 3) decreases in the number of Q-HRs with age are not due to the 'loss' of Q-heterochromatin on individual loci or chromosomes, but occur simultaneously in all the seven Qpolymorphic autosomes, in keeping with all our previous observations. It should be noted that decreases in the number of chromosomal QHRs with age do not occur smoothly apparently. Thus, according to our data, in the Kyrghyz and Russian populations $39.6 \%$ and $47.6 \%$ of chromosomal Q-HRs 'disappeared' by the age of 60 years, respectively. Of them the portion of new-born Kyrghyz and Russian infants amounted to $70.6 \%$ and $58.4 \%$, respectively. It is supposed that the lesser amount of Q-HRs in the genome of elderly subjects is due to the selective advantage in their survival to old age. The possible selective value of chromosomal Q-HRs is discussed. 
PO:08:42

\section{Chromosomal Q-heterochromatin variability in neonates deceased during first year of age.}

\author{
A. Ibraimov 1 \\ ${ }^{1}$ Laboratory of Human Genetics, National \\ Center of Cardiology and Internal Medicine, \\ Togolok Moldo str. 3, Bishkek, Kyrgyzstan, \\ 720040, C.I.S.
}

There is analyzed the amount of chromosomal Q-heterochromatin regions (Q-HRs) in a genome of neonates deceased during first years of life. Chromosome preparations were made from umbilical-cord blood with the subsequent Qstaining in 145 Kyrghyz and 37 Russian neonates. During the first year of life 17 Kyrghyz and 5 Russian neonates have deceased from various diseases in stationary conditions, the diagnosis of which is confirmed by pathologicalanatomical dissection. Mean numbers of Q-HRs per individual in newborn population were $3.16 \pm 0.13$ in Kyrghyz and $3.59 \pm 0.23$ in Russian, whereas in neonates died $=4.58 \pm 0.23$ and $=4.80 \pm 0.37$ respectively. Neonates are characterized by a high range of variability in the distribution of Q-HRs (from 0 up to 7). But died neonates, besides high value, differ by extremely narrow range of variability of Q-HRs in population: number of Q-HRs in a karyotype changes from 4 up to 6. Q-HRs in all samples studied are encountered with an expected frequency on all the potentially Q-polymorphic autosomes, i.e. in no group there was preferential Q-HR localization, and this is again suggesting that Q-heterochromatin is not locus-specific material in the genome. It is supposed that individuals with the greatest amount of Q-HRs in the newborn population have greater probability to die in the first years of life other conditions being equal.

\section{PO:08:43}

\section{Variability of chromosomal Q-heterochromatin regions in alcoholics and drug addicts.}
A. Ibraimov ${ }^{1}$

${ }^{1}$ Laboratory of Human Genetics, National Center of Cardiology and Internal Medicine, Togolok Moldo str. 3, Bishkek, Kyrgyzstan, 720040, C.I.S.

The quantitative variability of chromosomal Qheterochromatin regions (Q-HRs) was studied in alcoholics and drug addicts in various racial and ethnic groups. It was found that in the genome of alcoholics the amount of chromosomal Q-HRs is significantly lower than in control samples and drug addicts, whereas the latter have the greatest amount of chromosomal Q-HRs.

Alcoholics are characterized by the lowest value of the mean number of Q-HRs per individual in the population $(=1.45 \pm 0.09)$ and by the narrowest range of variability of the number of chromosomal Q-HRs (from 0 up to 4) among all the samples studied. Although subjects with drug abuse typically had the highest value $(4.1 \pm 0.11)$, nevertheless it should be noted that the range of variability of the number of Q-HRs was as narrow (from 2 to 6 ) in the as that in alcoholics. However prevailed individuals with great numbers of Q-HRs in their genome. We just draw attention to the fact that in the vulnerability of man to alcoholism and drug addiction is also of significance, in addiction to other things, the amount of chromosomal Q-HRs in his genome, since the values of absolute and relative Q-HR frequencies on Qpolymorphic autosomes in all samples are encountered with an expected frequency on all the potentially Q-polymorphic chromosomes, indicating that Q-heterochromatin is not locus-specific material in the human genome.

\section{PO:08:44}

\section{Variability of chromosomal Q-heterochromatin regions in patients with alimentary obesity}

\author{
A. Ibraimov ${ }^{1}$ \\ ${ }^{1}$ Laboratory of Human Genetics, National Center \\ of Cardiology and Internal Medicine, Togolok \\ Moldo str. 3, Bishkek, Kyrgyzstan, 720040, C.I.S. \\ Variability of the amount of chromosomal \\ Q-heterochromatin regions (Q-HRs) was studied \\ in individuals with alimentary obesity and in
}


controls from two ethnic groups living in Bishkek, Kyrghyzstan. It was shown that obese individuals differ from controls in the extremely low amount of Q-HRs in their genome. Namely, females with obesity, regardless of their ethnic origin, are characterized by a consistently low value of the mean number $(=1.30 \pm 0.08)$ and by narrow range of variability in the distribution of the Q-HR numbers in the samples (from 0 up to $3)$ as compared with controls $(=2.94 \pm 0.14$ and from 0 up to 7, respectively). The values of absolute and relative Q-HR frequencies on Q-polymorphic autosomes in obese individuals are encountered with an expected frequency on all the potentially Q-polymorphic autosomes, indicating, as we suppose, nonlocusspecifity of Q-HR in the human genome. The question as to the possible role of the amount of Q-HRs in the genome in the vulnerability of man to the development of alimentary obesity is discussed.

\section{PO:08:45}

\section{The age of the Philadelphia chromosome $(\mathrm{Ph})$ : dating the origin of tumour karyotype aberration.}

\section{G. Jankovic ${ }^{1}$, M. Colovic ${ }^{1}$, P. Wiernik ${ }^{2}$ and M. Andolina ${ }^{3}$}

${ }^{1}$ Institute of Haematology, Belgrade, Serbia and Montenegro; ${ }^{2}$ Our Lady of Mercy Cancer Center, New York, USA; ${ }^{3}$ Istituto per L'Infancia, Trieste, Italy

Neither the chromosomes fossilize nor the recurring chromosome translocations (CT) in human leukaemias accumulate over time (they are purged in each generation), so it is not obvious how could their frequencies in patient populations be used to age-date modern tumour karyotype. We assume that (i) CT have been selectively neutral during human evolution and that (ii) the evolutionary DNA dynamics (mutation) underlying the propensity for CT-formation, in association with leukaemogenic allele fusion, was essentially random in time. Based on proportions of the $\mathrm{Ph}$ polymorphism (+/-) associated with the $\mathrm{BCR} / \mathrm{ABL}$ oncogene fusion in myeloid [chronic myeloid leukaemia (CML), acute myeloblastic leukaemia (AML)] and acute lymphoblastic leukaemias (ALL) leukaemias 'age' estimates of the $\mathrm{Ph}$ itself were obtained. The incidence rates were entered in place of decay constant lambda) in a relation between exponential decay of Ph-negativity and time [t] elapsed since the $\mathrm{Ph}$ first appeared. The Ph-negativity characterized all $\mathrm{BCR} / \mathrm{ABL}$ fusions in the ancient state $[\mathrm{Ro}=1.0]$. Proportions of the Ph-negative variant of $\mathrm{BCR} / \mathrm{ABL}$ chimerism $[\mathrm{R}]$ were obtained as $\sim 10$ percent of the proportion of $\mathrm{Ph}-$ positive patients in analogy with CML where the Ph-negatives constitute $\sim 0.1$ of all patients. Using the equation $\mathrm{t}=1 /$ (lambda) $\ln \mathrm{Ro} / \mathrm{R}$, identical values for $\mathrm{t}$ (age of the $\mathrm{Ph}$ ) are obtained for otherwise biologically quite distinct but $\mathrm{Ph}$ positive leukaemias. The estimates of $\mathrm{t}$ (in years) are 230,258 (CML), 230,170 (AML), 231,229 (adult ALL), and 230,472 (paediatric ALL). The $\mathrm{Ph}$ age estimates $(\sim 230,000$ years $)$ concur with the 200,000 years estimated for the most recent common mitochondriarch.

\section{PO:08:46}

\section{Kuwait Chromosomal Abnormality Registry (KCR) (1979-2003) \\ D. Krishnamurthy ${ }^{1}$ and S. Al-Awadi ${ }^{1}$}

${ }^{1}$ Cytogenetic Laboratory, Kuwait Medical Genetics, Center, Maternity Hospital, Kuwait

The Kuwait Medical Genetics Center, was established in the year 1979, to provide Cytogenetic diagnostic services in the referred population suspected for chromosomal abnormalites. During the period from 1979-2003 chromosomal analysis has been carried out in 16,850 cases among the 2.3 million heterogeneous population. Cytogenetic analysis were carried out as per the standard procedures, and the karyotype was interpreted according to the ISCN. The registry includes the common numerical and structural as well as some very rare cases. One of the noteworthy observation in this population is recurrent regular trisomy 21 , a very high incidence of trisomy 21 (3.3 per 1000 births), clustering of trisomy 18 and cri-du-chat syndrome cases. The data presented is one of the largest series in the Middle East population. The significance of 
consanguinity, inbreeding and non-availability of prenatal diagnosis services (due to socio-cultural reasons) in relation to increased chromosomal abnormalities in this population will be discussed.

\section{PO:08:47}

\section{Population cytogenetics of Acrotylus} humbertianus (Sauss) (Fam: Acrididae; Sub.Fam: Oedipodinae) from South India

\author{
S. Mayya ${ }^{1}$, J. Hegde ${ }^{1}$ and S. Kanale ${ }^{2}$ \\ ${ }^{1}$ Department of Biosciences, Mangalore \\ University, Mangalagangotri, Mangalore, India; \\ ${ }^{2}$ Department of Applied Zoology, Mangalore \\ University, Mangalagangotri, Mangalore, India
}

Meiotic chromosomal analysis of Indian short horned grasshopper Acrotylus humbertianus from three natural populations, Maravanthe, Someshwara and Calicut in the west coast of South India has been studied. As per the data on background gamma radiations in the west coast (Radhakrishna et al. 1993), it is found that the three study areas experience varied levels of radiations. i.e. Maravanthe, 3-5nGyh-1; Someshwara 215nGyh-1; Calicut 56nGyh-1. Hence it was proposed to carryout chromosomal studies using testis material on commonly available grasshoppers. The study revealed different kinds of chromosomal anomalies which include stickiness and clumping (16.24\%), bivalent segregation $(10.84 \%)$, chromosome bridges $(5.96 \%)$, laggards $(5.42 \%)$, polyploidy $(1.48 \%)$, gaps and breaks $(0.98 \%)$ etc. in different degrees in all the three populations. The frequencies of these anomalies in relation to background radiation have been discussed.

\section{PO:08:49}

\section{Incidence of chromosomal}

abnormalities; A prospective study in a referrd Iranian population.

A. Movafagh ${ }^{1}$, Z. Pirzadeh ${ }^{2}$, H. Moosavi ${ }^{1}$, S. Ayenehchi ${ }^{1}$, M. Ghasemi Barghi ${ }^{2}$ and $H$. Javadi ${ }^{2}$
${ }^{1}$ Shahid Beheshti Medical University, Department of Genetics, Evin Ave, Tehran, Iran.; ${ }^{2}$ Ghazvin Medical University, Ghazvin, Iran

We report the results of an analysis of congenital malformations and genetic diseases of 24513 children from infancy to age 19 years, each with varying clinical manifestation from normal to severe congenital anomalies including metabolic, heart, blood, infertility, neurology, deafness, made over 5 years period in a major pediatric hospital and genetic division centre Ghazvin state, and results of 76 cases drawn from this work, Taleghani hospital Tehran. In this survey, 78 cases with abnormal chromosomal have been investigated employing conventional cytogenetic GTG- technic by chromosome banding. Down's syndrome was the most common anomalies followed by Patau-Edward's-Wolf Hirosh Horn syndromes among autosomes. Among sex chromosome, Turner-KFS-super female-super maleFragile $\mathrm{X}$ syndromes, was the most common. This date gives an idea of the spectrum of cytogenetic disorders seen in Iran from this region, more detail will be discussed.

\section{PO:08:50}

\section{FRAXA leading mutational pathways among Basques}

O. Peñagarikano ${ }^{1}$, M. Télez ${ }^{1}$, B. Ortega1, L. Valverde ${ }^{1}$, P. Flores ${ }^{2}$, B. Criado ${ }^{3}$ and I. Arrieta ${ }^{1}$

${ }^{1}$ Dpto. Genética, Antropología Física y Fisiología Animal, Facultad de Ciencia y Tecnología, Universidad del País Vasco, Bilbao, Spain; ${ }^{2}$ Dpto. Farmacología Clínica y Dietética, Escuela de Enfermería, Universidad del País Vasco, Bilbao, Spain; ${ }^{3}$ Instituto Portugués de Oncología, Porto, Portugal

Fragile sites are heritable points on a chromosome where gaps and breaks tend to occur. They are mainly designated as common or rare based on their frequency of expression. In addition, they are subdivided according to the specific culture media needed for their expression. FRAXA, a fragile site located in Xq27.3, is a rare folatesensitive fragile site associated to fragile $\mathrm{X}$ syndrome (MIM\#309550). Fragile $\mathrm{X}$ syndrome is 
the most common form of inherited mental retardation. The publication in 1991 of both the molecular basis of the fragile site and the syndrome, the massive expansion of a CGG repeat in the FMR1 X linked gene, focused much attention on trinucleotide repeat expansion as a mutational mechanism. In addition, even the slight expansion of this microsatellite sequence is associated to other disorders such as premature ovarian failure (POF) and tremor/ataxia syndrome (TAS). The most important factor that influences on the trinucleotide repeat expansion is the length of the repeat as well as its internal structure (the presence/absence of anchoring interruptions). In order to ascertain the stability of the FMR1 CGG repeat in the Basque Country we have analyzed these factors in 158 healthy males from the Spanish Basque population. Basques may represent one of the oldest human isolates. As a result, they show distinctive cultural and genetic characteristics. The data show a lower prevalence of large CGG alleles among this population. However, analysis of the AGG interspersion pattern suggests the existence of different mutational pathways that could lead to longer CGG repeats.

\section{PO:08:51}

\section{Cytogenetic findings in 39 schizophrenic patients}

Y. Tarkan-Argüden ${ }^{1}$, S. Yilmaz ${ }^{1}$, A. Egriboz ${ }^{1}$, B. Gunel ${ }^{2}$, A. Yilmaz ${ }^{2}$ A. Duran ${ }^{2}$ and S. Hacihanefioglu ${ }^{1}$

${ }^{1}$ Dept. of Medical Biology, Istanbul University, Cerrahpasa Medical School, 34300, Istanbul, Turkey; ${ }^{2}$ Dept. of Psychiatry, Istanbul University, Cerrahpasa Medical School, 34300, Istanbul, Turkey

Schizophrenia is a common and serious psychiatric illness with peak age at onset in early adulthood. Family twin and adoption studies have demonstrated that schizophrenia is predominantly genetically determined and has high heritability. Although many linkage and association studies have been performed, no gene has yet been established as giving major susceptibility to schizophrenia. The use of chromosomal examinations could be an alternative or complementary approach to identify a susceptibility locus for schizophrenia. Chromosomal abnormalities may be helpful to find not only canditate regions for linkage studies, but also valuable materials for positional cloning. We performed cytogenetic examinations on peripheral lymphocytes of 39 (22 women, 17 men) schizophrenia patients. Gbanded metaphases were examined according to the ISCN (1995). One male and one female patients revealed only normal karyotypes. There were not good quality metaphases to examine in two cases. In 23 cases, there were non-recurrent chromosomal abnormalities along with normal karyotypes. In 12 cases, we observed recurrent chromosome abnormalities which include $\operatorname{del}(\mathrm{X})(\mathrm{q} 26 \mathrm{q} 27), \quad \operatorname{dup}(6)(\mathrm{p} 22) ?, \quad \operatorname{add}(22)(\mathrm{q} 12)$ ?, $-\mathrm{X}$, + mars, $-15,-22$ (in two cases), der(7)?, $-18, \operatorname{del}(9)(\mathrm{q} 12),-21, \operatorname{der}(21)$ (in two cases), $\operatorname{der}(1), \operatorname{add}(4)(\mathrm{q} 35 ?)$.

This work was supported by the Research Fund of Istanbul University. Project Number: 1619/30042001

\section{PO:08:52}

\section{Fragile site expression in hypertensive patients treated with atenolol}

\section{Télez ${ }^{1}$, O. Peñagarikano ${ }^{1}$, B. Criado², C. Lostao ${ }^{1}$, P. Flores ${ }^{3}$, E. Ortiz ${ }^{4}$ and I. Arrieta ${ }^{1}$}

${ }^{1}$ Dpto. Genética, Antropologia Fisica y Fisiologia Animal, Facultad de Ciencia y Tecnologia, Universidad del Pais Vasco, Bilbao, Spain; ${ }^{2}$ Instituto Portugués de Oncologia, Porto, Portugal; ${ }^{3}$ Dpto. Farmacologia Clinica y Dietética, Escuela de Enfermeria, Universidad del Pais Vasco, Bilbao, Spain; ${ }^{4}$ Dpto. Especialidades Médico-Quirírgicas, Universidad del Pais Vasco, Bilbao, Spain

FS are specific points of chromosomes at which the chromosome is liable to break. The latest compendium comprises over 117, classified as common and rare according to their relative occurrence in the population. Both common and rare FS are further subdivided according to the tissue culture conditions required for their expression. Their location has been correlated cytogenetically with locations of recurrent translocation and deletion breakpoints in cancer cells 
as well as the integration sites of oncogene loci. Previous studies have shown that genotoxic agents increase the expression of FS.

The analysis of the FS significantly expressed in our sample allowed the identification of possible specific FS on chromosomes of the hypertensive patients. On one hand, the analysis of FS expression defined 28 different bands in patients and 21 in controls considered to be FS in the sample; FRA3B, FRA6C, FRA7C and FRA16D were the four most frequently expressed in both groups. On the other hand, FRA1E, FRA1F, FRA2D, FRA5D, FRA7B, FRA7H, FRA11G, FRA11H, FRAXB, FRAXC and the band 17q12-21 were FS exclusively expressed in patients. The band 17q12-21, where known FS have not been reported, was in the fifth position in order of expression and was expressed in $63.64 \%$ of patients.

Until now, linkage analysis has been pursued to identify hypertension susceptibility genes but results have been difficult to reconcile. One notable exception is the study by Lathrop et al., which provides reasonably compelling evidence for an hypertension susceptibility locus on chromosome 17. Our results seem to support that finding.

\section{PO:08:53}

\section{Cytogenetic investigations of natural triploid birch hybrids in Iceland}

\section{A. Thorsson ${ }^{1}$ and K. Anamthawat-Jonsson ${ }^{1}$}

${ }^{1}$ University of Iceland, Department of Biology, Askja, Sturlugata 7, Reykjavik 101, Iceland

Triploid hybrids (42 chromosomes) between tetraploid downy birch (Betula pubescens) and diploid dwarf birch (B. nana) are found in about $10 \%$ of birch plants in birch woodlands throughout Iceland. The two species co-exist in Iceland and in northern parts of Europe, and due to short growing season their flowering periods overlap significantly and the species hybridize. Crossing experiments produce triploid interspecific hybrids with high frequency, especially when $B$. nana is the seed parent. Ribosomal gene mapping by fluorescent in situ hybridization (rDNA-FISH) confirms the presence of parental genomes in the hybrids. The hybrid plants have intermediate leaf characters and growth form. As they are not completely sterile, they can produce morphologically variable progeny after backcrossing. However, these plants are not variable in their chromosome numbers - they are diploid, triploid and tetraploid. No aneuploids have ever been found, suggesting genetic control over meiosis or strict gametic selection in the hybrids. In this study we characterize the genomes in natural triploid hybrids using rDNA-FISH mapping together with Southern genomic hybridization and cpDNA-RFLP analysis. We also test our hypothesis that the selection against aneuploidy in the hybrids is during meiosis, by looking at chromosome behaviour and microsporogenesis. Triploid hybrids seem to produce two sizes of viable gametes (pollen), possibly corresponding to genome sizes as predicted. The significance of interspecific hybridization is probably in maintaining genetic variability and gene flow between the two species, via introgression. Such mechanism may be the major force behind the species survivability in harsh environments like in Iceland.

\section{Solid Tumours Symposium}

\section{L138}

\section{Cancer cytogenetics: potentialities and limitations of the current technology}

\section{H. Tanke ${ }^{1}$}

${ }^{1}$ Department of Molecular Cell Biology; Leiden Medical Centre; Leiden; Netherlands

Conventional cytogenetic investigations of Giemsa banded metaphase chromosomes generated from cultured solid tumours have been hampered by both biological as well technical problems. Biological factors relate to tumour cell heterogeneity and cell culturing artefacts (c.q. selection), whereas major technical obstacles are encountered with respect to poor quality (or no!) metaphase chromosomes, insufficient banding resolution and very complex rearranged karyograms.

Techniques developed in the past two decades such as 48 colour FISH karyotyping, comparative genomic hybridisation (chromosomal as well as array $\mathrm{CGH}$ ) and culturing techniques that 
produce high quality metaphases by chemical induced PCC (premature condensation of chromosomes), justify a revisit of the field of cancer cytogenetics. This paper outlines the state-of-art of the current technology illustrated by selected samples from routine clinical practice (mainly soft tissue tumours).

\section{L139}

\section{Genome wide approaches (high resolution BAC array CGH and SAGE) for analysis of lung cancer}

C. Macaulay ${ }^{1}$, K. Lonergan ${ }^{1}$, C. Garnis ${ }^{1}$, B. Coe ${ }^{1}$, S. Zuyderduyn ${ }^{1}$, M. Marra ${ }^{1}$, A. Gazdar', M. Tsao ${ }^{3}$, S. Lam ${ }^{1}$ and W. Lam ${ }^{1}$

${ }^{1}$ British Columbia Cancer Research Centre, Vancouver, British Columbia, Canada;

${ }^{2}$ University of Texas Southwestern Medical Centre, Dallas, Texas, USA; ${ }^{3}$ Ontario Cancer Institute, Princess Margaret Hospital, Toronto, Ontario, Canada

Genetic alterations and expression changes are known to be associated with the neoplastic process in the lung. Recently developed tools enable the genomic wide evaluation of genetic copy number changes (at a resolution 10-50 times greater than conventional array $\mathrm{CGH}$ ) and known gene and novel gene expression changes (SAGE) from minute lung samples.

The objectives of our study were (1) To examine the data from a CGH array designed to provide complete coverage of the human genome. (2) To identify genes important to disease progression (in 40 plus neoplastic lung specimens). (3) Correlation of these with changes seen in 40 plus SAGE lung libraries. (4) Evaluate candidates on a recently developed cytology microarray platform.

Arrays of 32,433 amplified BAC DNA clones spanning the whole genome has been constructed. Experimental conditions for array CGH for clinical materials and the impact of tissue heterogeneity and DNA quality/quantity in the generation of an accurate array $\mathrm{CGH}$ profile have been assessed. This has identified recurrent genetic alterations. In addition, the tiling resolution of the array facilitated the detection of micro-amplification of known and novel genes. The repeatability and robustness of SAGE on limited lung samples has been demonstrated. More than $50014 \mathrm{mer}$ tags of which 411 mapped to genes were found to be more than 50x over expressed between normal and cancer tissue. A small subset of the possible screening markers have been evaluated on a set of cytology microarrays. The performance of a few of these markers approach that required for a usable screening test.

\section{L141}

\section{Chemically induced chromosome condensation technique in tumor cytogenetics}

\author{
K. Szuhai ${ }^{1}$ \\ ${ }^{1}$ Department of Molecular Cell Biology, Leiden \\ University Medical Center, Leiden, \\ The Netherlands
}

In the last decade several new molecular techniques have been developed to identify genomic alterations associated with tumor development and progression. However, there is no such an approach that allows genome-wide screening for unknown chromosomal alterations with balanced rearrangements. These limitations necessitate the use of classical cytogenetic approaches to visualize chromosomal alterations. However, several tumor types characterized with low mitotic rate or unable to adapt to the culture conditions, which results in the lack of tumor cell metaphase or in the metaphases from the contaminating normal cells. An alternative technique that induces a rapid condensation of chromosomes independent of the numbers of M-phase cells might be a solution to these problems. Cell cycle independent condensation of chromosomes can be achieved by the chemical premature chromosome condensation (cPCC) technique. This approach will particularly valuable to study tumor cells with a slow transition from G2 to M phase. The value of the cPCC technique was evaluated on a set of 60 solid tumors, bone marrow and peripheral blood samples of hematological malignancies. The use of chemically induced chromosome con- 
densation was found to be the most informative in tumors with slow growth rate resulting in 4-10 fold increase of the number of tumor metaphases compared to the classical Colcemid harvesting. This technique can easily adapted to fit routine practice, cPCC in combination with multicolor FISH is considered as an important tool in tumor cytogenetics in addition to classical cytogenetic investigation.

\section{L142}

\section{Profiling of chromosomal imbalances as a tool for prognostic stratification and gene discovery in cancer}

\section{F. Speleman ${ }^{1}$, N. Van Roy ${ }^{1}$, B. Menten ${ }^{1}$, A. De Paepe ${ }^{1}$, G. Laureys ${ }^{2}$ and J. Vandesompele ${ }^{1}$}

${ }^{1}$ Center for Medical Genetics, Ghent University Hospital, Ghent, Belgium; ${ }^{2}$ Dept of Pediatric Oncology, Ghent University Hospital, Ghent, Belgium

Neuroblastoma (NB) is a paediatric neural crest derived tumour, that shows a remarkably heterogeneous clinical behaviour. Karyotyping typically showed 1p-deletions and MYCN amplification but was in the majority of cases only applicable to high stage NB. Molecular studies, mainly investigating loss of heterozygosity, lead to identification of other chromosomal regions of recurrent deletions involving chromosome regions $3 p$, $4 p, 11 q$ and 14q. Molecular cytogenetics introduced new possibilities for the genetic analysis of $\mathrm{NB}$, revealing a high incidence of $17 \mathrm{q}$ gain due to unbalanced chromosome 17 translocations in NB cell lines and high stage tumours. Comparative genomic hybridisation $(\mathrm{CGH})$ provided a genome wide view on the genomic imbalances associated with all clinical subtypes of NB. Statistical analysis of $\mathrm{CGH}$ data from a large cohort of patients clearly demonstrated the presence of three major subgroups. Furthermore, chromosome $17 \mathrm{q}$ gain and normal chromosome 17 status were shown by multivariate analysis to be the strongest independent prognostic parameters for poor outcome. More recently, using highresolution copy number analysis by arrayCGH analysis and SNP chip analysis we were able to detect new recurrent genomic imbalances that might lead to the discovery of genes involved in normal neuroblast differentiation and NB development.

In summary, our work and that of others illustrates that, together with current efforts of gene expression profiling, the study of the genomic alterations occurring in tumours (in casu NB) provides critical prognostic information and also contributes to the identification of candidate NB genes.

\section{L144}

\section{MAC (Mammalian Artificial}

\section{Chromosome) based elimination test in the functional identification of tumor growth antagonizing genes}

\author{
A. Szeles ${ }^{1}$, G. Hadlaczky ${ }^{2}$ and S. Imreh ${ }^{1}$
}

${ }^{1}$ Karolinska Institutet, MTC Karolinska Inst.

MTC, Stockholm, Sweden; ${ }^{2}$ Institute of Genetics, BRC, Szeged, Hungary

We have developed a functional test system; the microcell hybrid $(\mathrm{MCH})$ based 'elimination test' (Et). The MCHs are tested for the elimination or retention of specific human chromosome 3 (chr.3) regions after passages in SCID mice. We have defined a commonly eliminated region (CER). We have covered these regions with PACs (bacteriophage P1 based artificial chromosome) and identified several genes.

Our aim is to reintroduce the PACs and genes derived from the CER into cancer cell lines. For this purpose we are using mammalian artificial chromosome (MAC) vectors. Our MACs are satellite DNA based artificial chromosomes (SATACs). Among the genes identified on CER lactoferrin (LF) is one of the candidates. We have constructed human LF carrying mouse SATACs (mSATAC-hLF) in a mouse (LMTK) and human LF carrying human SATACs (hSATAC-hLF) in a human fibrosarcoma lines (HT1080). The mSATAC-hLF carrying mouse and hSATAC - hLF carrying human fibrosarcoma lines were used for studies of the effect of the hLF to in vitro and in vivo tumour growth. Our results have showed that the exogenous hLF had no consistent effect on in vitro growth. mSATAC-hLF was maintained in 
$78 \%$ and hSATAC-hLF in $92.5 \%$ of cells in vitro. Next, we have tested the cells in SCID mice. We have found that the majority of cells in SCID derived tumors lost the SATAC-hLF. It can be concluded, that elimination of the exogenous hLF carrying SATACs provides to the mouse and human fibrosarcoma cells with in vivo growth advantage.

\section{L145}

\section{The statistical behavior of complex cancer karyotypes}

\section{Häglund ${ }^{1}$, A. Frigyesi ${ }^{2}$, T. Sall ${ }^{3}$, D. Giselsson ${ }^{1}$ and F. Mitelman ${ }^{1}$}

${ }^{1}$ Department of Clinical Genetics, University Hospital, Lund, Sweden; ${ }^{2}$ Centre for Mathematical Sciences, Mathematical Statistics, Lund University, Sweden; ${ }^{3}$ Department of Genetics, Lund University, Sweden

Epithelial tumors commonly show complex and variable karyotypes obscuring the identification of general patterns of the karyotypic evolution. To overcome some of these problems, we have previously analyzed systematically the accumulated cytogenetic data for individual tumor types using various statistical means. In the present investigation we perform several meta-analyses of data obtained from a number of epithelial tumors, including head and neck, kidney, bladder, breast, colorectal, ovarian, and lung cancer, as well as malignant melanoma, and Wilms tumor, with the specific aim to disclose common patterns of the karyotypic evolution. We show that the tumors frequently develop through a hypo- or a hyperdiploid pathway and progress by an increasing number of alternative imbalances through at least two karyotypic phases, Phase I and Phase II, and possibly a third, Phase III. During Phase I the karyotypes exhibit a power law distribution of the number of changes per tumor as well as a Zipf distribution of the frequency by which bands are involved in breaks. At the transition from Phase I to Phase II/III the observed power law and Zipf distributions are lost indicating a transition from an ordered and highly structured process to a disordered and chaotic pattern. The change in karyotypic orderliness at the transition from Phase I to Phase II/III is also evident by a drastic difference in karyotypoic entropy.

\section{L146}

\section{Sequence copy number changes in Barrett's adenocarcinoma: Array-CGH and quantitative Sequential Multi-Locus (SML-) FISH analysis}

B. Albrecht ${ }^{1}$, K. Bäwering ${ }^{2}$ H. Zitzelsberger ${ }^{3}$ T. Wiech ${ }^{1}$, M. Hausmann ${ }^{1}$, H. Häfler ${ }^{2}$, M. Werner ${ }^{1}$ and A. Walch ${ }^{1}$

${ }^{1}$ University Hospital Freiburg, Institute of Pathology; Freiburg i. Br., Germany; ${ }^{2}$ Technical University of Munich, Institute of Pathology; Munich, Germany; ${ }^{3}$ GSF-National Research Center for Environment and Health, Institute of Molecular Radiation Biology; Neuherberg, Germany

Array-CGH (aCGH) allowed the identification of DNA sequence copy number changes in formalin-fixed, paraffin-embedded tissue samples of Barrett's adenocarcinomas (BCA). The microarrays used contained 287 genomic targets covering oncogenes, tumor suppressor genes and DNA sequences localized within previously reported changed chromosomal regions in BCA. DNA sequence copy number gains and losses were identified for a panel of approximately 50 genes, mostly so far not described in BCA. For a first validation, the DNA sequence copy number changes of selected clones (SNRPN, CMYC, CErbB2, ZNF217) were confirmed by fluorescence in situ hybridization (FISH). However, the results were obtained from DNA solutions representing an average DNA over a lot of BCA cells neglecting individual cellular variability and complexity. Therefore, aCGH was a starting point for further systematic microscopic studies of the numerical organization of gene loci and genome architecture in BCA. Selected cases were used for systematic studies by 3D confocal laser scanning microscopy after sequential multi-locus FISH (SML-FISH). Different states of aneuploidia of the chromosomes 7, 8, 11, 17, 20 were analyzed 
in correlation to the copy number changes of the oncogenes CMET, CMYC, CyclinD1, CErbB2, and 20.q13.2. Due to SML-FISH it was possible to detect simultaneously existing copy number changes of these genes and coincident intra-cellular aneuploidia in individual cells. The correlation of the analyzed parameter indicated the complex changes in the genome and supported findings of intra-tumor heterogeneity being typical for Barrett's adenocarcinoma.

\section{L147}

Comparative genomics and chromosome deletions in cancer. Lessons of the microcell hybrid based 'elimination test'

\section{S. Imreh $^{1}$}

${ }^{1}$ Karolinska Institutet, MTC, Stockholm, Sweden

The elimination test developed by us is based on the transfer of single human chromosomes into tumor cells of human or murine origin, via microcell fusion. The microcell hybrids are tested for the elimination vs. retention of specific chromosome regions after one or several passages in SCID mice. On chromosome 3 the group gradually narrowed down a common eliminated region on 3p21.3 designated CER1. A common retained region (CRR) preferentially retained after 4 consecutive SCID passages has been identified at 3q26-qter. It contains among others the p53 induced WIG1 gene and the RNA component of telomerase. In parallel we proved that in human/ human $\mathrm{MCHs}$ based Et similar regions are eliminated as in the human/murine system and CER1-LOH characterizes $83 \%$ of human tumors.

The transcriptional map of CER $1 \& 2$ has been assembled, that contain 26 genes 6 of them novel, cloned by the group. LIMD1 and LF are tumor inhibitory gene candidates on functional basis but the presence of $10 \mathrm{CCRs}$, that arised by gene duplications, in CER1\&2 is the most provocative. We described the mouse synteny of $3 p$ deletions and found that contains both the borders of conserved segments and a hairpin forming (TATAGA) ${ }^{\mathrm{n}}$ repeat. Invertebrates (Caenorhabditis elegans, Drosophila) in contrast with vertebrates (Fugu,
Mus) show zevolutionary plasticityä around the mouse/human conservation breakpoint, that may explain the regional involvement in cancer related deletions.

Yang Y et al. PNAS 98, 1136-1141, 2001; Kost-Alimova M et al, $P N A S$ 100:6622-6627, 2003

\section{PO:08:54}

\section{A clue for recessive inheritance pattern in familial colorectal cancer in Iranian population}

\author{
F. Bishehsari ${ }^{1}$, M. Mahdavinia ${ }^{1}$, R. Ansari ${ }^{1}$ \\ and R. Malekzadeh ${ }^{1}$ \\ ${ }^{1}$ Digestive Disease Research Center, Shariati \\ Hospital, Kargar Shomali, 14114, Tehran, Iran
}

The incidence of colorectal cancer in Iranian young adults is growing, the aim of this study was to investigate the familial aggregation of colorectal cancer and also evaluate the hereditary pattern of cancer in these families.

In a pilot study, we conducted detailed interviews with 327 Iranian colorectal cancer patients about their family histories of malignancies, age at diagnosis and consanguinity.

Overall 118 patients $(32 \%)$ had at least one first-degree relative with cancer. Twenty five patients $(7.6 \%)$ had more than two first- or second-degree relatives with colorectal or other HNPCC related cancers; suggestive of Lynch I or II by the Amsterdam II criteria. Examining the family pedigrees, most of the family clustering of disease was suggestive of autosomal dominant pattern of inheritance, but two families showed different patterns. One family with history of colon and gastric cancer in father's grand father and early-onset colon cancer in mother's mother and endometrial cancer in two of mother's aunts, but no history of any cancer in second generation, parents are first cousins while four of nine, in third generation (their children) had early-onset colon cancer. The other family with colon and extra colonic cancers in both side grandparents relatives and no history of such disease in parents, in third generation two colon and one breast cancer before age of forty, parents were not biologically related. 
These results suggest that recessive contributions could be responsible in some family clusters of colorectal cancer, which needs further molecular assessment of $\mathrm{CRC}$ related genes.

\section{PO:08:55}

Oesophageal carcinoma in South Africa: Possible involvement of FHIT

\section{J. Brown ${ }^{1}$, A. Stafne ${ }^{1}$, S. Engelbrecht ${ }^{1}$,} R. Veale $^{1}$ and P. Willem ${ }^{1}$

${ }^{1}$ University of the Witwatersrand, Johannesburg, SA

Oesophageal cancer (OC) is the third most common malignancy in South Africa (SA), affecting 1 in 20 and 1 in 76 black males and females respectively. The dominant type is squamous cell carcinoma (SSC), an aggressive disease showing a poor prognosis largely due to late diagnosis after the onset of symptoms. The precise mechanisms of development and progression of this disease are still unclear and the identification of genetic changes associated with these tumours may shed light on its aetiology in SA, as well as provide early diagnostic tools. The Fragile Histidine Triad gene (FHIT) is a tumour suppressor gene encompassing the most common fragile site FRA3B, located at $3 p 14.2$. Loss of heterozygosity at the FHIT locus has been detected in a high percentage of primary tumours and pre-cancerous lesions of the oesophagus elsewhere, suggesting that it maybe an early event in oesophageal carcinogenesis. This gene has not been investigated in SA oesophageal cancer. We looked at the integrity and expression of FHIT in 5 oesophageal cancer cell lines established in SA. Due to heterogeneous deletions within FHIT, we used fluorescence in situ hybridisation with FHIT specific probes as a screening tool for gene integrity. Significant rearrangements of the FHIT gene were detected in 2 of the cell lines. RT-PCR detected aberrant transcripts in the same 2 cell lines. These findings suggest that FHIT may have a role to play in oesophageal carcinogenesis in SA patients. Investigation of FHIT's role in OC in SA patients is pertinent.

\section{PO:08:56}

\section{Cytogenetic and molecular cytogenetic analyses in brain tumors}

\author{
A. Cirakoglu ${ }^{1}$, Y. Tarkan-argüden ${ }^{1}$, \\ S. Yilmaz ${ }^{1}$, D. Kuru' ${ }^{1}$, M. Inan' ${ }^{2}$, M. Uzan' \\ E. Ozyurt ${ }^{2}$ and S. Hacihanefioglu ${ }^{1}$ \\ ${ }^{1}$ Dept. of Medical Biology, Cerrahpasa \\ Medical School, Istanbul University, Istanbul, \\ Turkey; ${ }^{2}$ Dept. of Neurosurgery, Cerrahpasa \\ Medical School, Istanbul University, Istanbul, \\ Turkey
}

There are reports in literature on chromosome abnormalities specific to different brain tumor subgroups. We set up tumor tissue cultures of 37 cases who had been operated due to brain tumors. Chromosomes were obtained from 16 cases, pathologically diagnosed as 3 astrcytomas, 2 oligodendrogliomas, 1 oligoastrocytoma, 3 neuroglial tumors, and 7 meningiomas. There was not a common numerical chromosome abnormality in astrocytoma cases. The breakpoints involved in structural chromosome abnormalities of the astrocytoma cases were; $3 \mathrm{q} 28$ in one case, 1p36, 1q31, 2q37, 3p21, 6q14, 7q36, 8q21q24, 9p24,9q10, 11p15, 11q23,16q24 in one case, and $\mathrm{Xq} 27,3 \mathrm{p} 21,7 \mathrm{p} 22,10 \mathrm{p} 15,22 \mathrm{q} 13,19 \mathrm{p}$ or $\mathrm{q} 13$ in another case. dmins and ring chromosomes were obtained in two cases. - $\mathrm{X}$ was the only clonal chromosome abnormality in one oligodendroglioma case. $-\mathrm{Y},-21$, and -22 were present in the oligoastrocytoma case. Aneuploidies in meningiomas were, -22 (in 5 cases), $-\mathrm{X},-10$ (in 3 cases each), $-3,-5,-6,-9,-12,-16$, $-17,-19,-20,-21$ (in 2 cases each), and -2 , $-4,-8,-11,-14$ (in one case each), $+3,+5$, $+9,+11,+14$ in one case and $+1,+13$, and +16 in another case. The structural abnormalities found in meningiomas were $\operatorname{del}(1)(\mathrm{p} 21)$ in one case, $\operatorname{inv}(2)(\mathrm{p} 15 \mathrm{q} 11)$, $\operatorname{add}(7)(\mathrm{q} 36)$ in another case. Interphase FISH using alpha-satellite probes specific to chromosomes $\mathrm{X}, \mathrm{Y}, 7$, and 10 was performed 16 cases and obtained results from 7 cases. We observed one case with +7 and -10 , one case with +7 , and one case with -10 .

This work was supported by the Research Fund of the University of Istanbul, project No. T-1056/19022001 
PO:08:58

\section{Comparison of fluorescence in situ} hybridization (FISH) and melanoma antigen gene (MAGE) Tests for the detection of primary bladder cancer

S. Koo' ${ }^{1}$, T. Jeong' ${ }^{1}$, J. Kang ${ }^{1}$, K. Kwon ${ }^{1}$, J. Park ${ }^{1}$, Y. $\mathrm{Na}^{2}$ and J. Lim ${ }^{2}$

${ }^{1}$ Department of Clinical Pathology, Chungnam National University Hospital, Taejon, South Korea; ${ }^{2}$ Department of Urology, Chungnam National University Hospital, Taejon,

South Korea

Our aim was to evaluate and compare the UroVysion (Vysis, USA) fluorescence in situ hybridization (FISH) and Melanoma Antigen Gene (MAGE) tests for improved detection of bladder cancer in urine specimens.

Forty first-morning urine specimens, 27 with a history of urothelial carcinoma and 13 with benign prostatic hyperplasia (controls), were analyzed. A mixture of fluorescent labeled probes to the centromeres of chromosomes 3, 7 and 17, and band 9p21 (P16/CDKN2A gene) was used for FISH to assess urinary cells for chromosomal abnormalities indicative of malignancy. In addition, reverse transcriptase-polymerase chain reaction (RT-PCR) and the nested PCR with the MAGE common primers $(\mathrm{C} 1 / \mathrm{C} 2$, and $\mathrm{C} 3 / \mathrm{C} 4)$ designed to detect MAGE 1-6 genes were conducted in the same samples and confirmed with the form of band on agarose gel electrophoresis.

Twenty-five of the 27 patients showed FISHpositive analysis. The sensitivity of FISH for pTa, pTis and pT1-pT4 tumors was $60 \%, 94 \%$ and $90 \%$, respectively, with an overall sensitivity of $93 \%$, compared to $55 \%, 88 \%$ and $87 \%$, respectively, for MAGE, with an overall sensitivity of $85 \%$. FISH was significantly more sensitive than MAGE for all tumors $(p=0.045)$. The specificity of FISH and MAGE among patients with benign prostatic hyperplasia was $96 \%$ and $95 \%$, respectively ( $p=0.627)$.

The sensitivity, but not specificity, of FISH for the detection of urothelial carcinomas is superior to that of MAGE. Even though further prospective studies are required, FISH and MAGE have the potential to significantly improve the detection of urothelial carcinoma.

\section{PO:08:59}

\section{Incidence and characteristics of $\mathrm{Ph}$ - negative in chronic meyloid lukemia}

\author{
A. Movafagh ${ }^{1}$, F. Isfahani ${ }^{1}$, H. Attarian ${ }^{1}$, \\ A. Hajfathali ${ }^{1}$, M. Ghadiani $^{1}$, S. Baharizadeh ${ }^{1}$ \\ and N. Varma ${ }^{2}$ \\ ${ }^{1}$ Shahid Beheshti Medical University, Tehran, \\ Iran; ${ }^{2}$ PGI, Chandigareh, India
}

Philadelphia $(\mathrm{Ph})$ chromosome negative chronic myeloid leukemia (CML) can be distinguished from clinically similar disorders on the basis of presence of rearrangement of the breakpoint cluster region (bcr) of chromosome 22. The leukemic cells of most patients with CML carry a $\mathrm{t}(9 ; 22) \quad(\mathrm{q} 34 ; \mathrm{q} 11)$. Standard cytogenetic analysis was performed on bone marrow or peripheral blood samples cultured for $0-48$ hours in the absence of mitogen, using G-banding technic. Of the $150 \mathrm{CML}$ cases analysed 81 were males and 69 females, the median age of these patients, at the time of cytogenetic examination, was 31(14 to 73 years), 140 cases $(93.3 \%)$ were Ph-positive and the remaining 10 cases $(6.6 \%)$ were Ph-negative. All of the $10 \mathrm{Ph}$-negative cases, except 2 cases with chromosomal abnormalities showed a normal karyotype. The incidence of Ph-negative cases, which was low (6.6\%), was considerably lower than usually reported incidence of about $15 \%$, though it has been pointed out that the incidence varies from $0 \%$ to more than $30 \%$ among different publications. Preliminary evidence suggests that these cases respond to chemotherapy and behave clinically as typical $\mathrm{Ph}$-positive CML.

\section{PO:08:60}

\section{Molecular cytogenetic abnormalities in Thai colorectal tumors by comparative genomic hybridization technique}

S. Poeaim ${ }^{1}$, B. Rerkamnuaychoke ${ }^{2}$, S. Jesdapatarakul ${ }^{3}$ and A. Campiranon ${ }^{4}$ 
${ }^{1}$ Department of Applied Biology, Faculty of Science, King Mongkut's Institute of Technology Ladkrabang. Bangkok 10520, Thailand;

${ }^{2}$ Department of Pathology, Faculty of Medicine, Ramathibodi Hospital, Mahidol University, Thailand; ${ }^{3}$ Department of Pathology, Vajira Hospital Medical College, Thailand; ${ }^{4}$ Department of Genetics, Faculty of Science, Kasetsart University, Thailand

Little information is know about the genetic changes of colorectal cancer (CRC) in Thailand. The aim of the present study was to identify copy number aberration of potential oncogene and tumor suppressor gene locations in CRC. Comparative Genomic Hybridization (CGH) technique was used to screen 40 cases of CRC that were divided into 3 stages: Dukes' A $(\mathrm{n}=11)$; Dukes' B $(\mathrm{n}=12)$ and Dukes' $\mathrm{C}(\mathrm{n}=17)$. The most frequent gains in all $\mathrm{CRC}$ were on chromosome arms 16p (15\%: 6 of 40 tumors), $7 \mathrm{p}, 7 \mathrm{q}, 12 \mathrm{q}, 13 \mathrm{q}, 17 \mathrm{q}(17.5 \%$ each $), 19 \mathrm{p}(20 \%)$, $19 q(22.5 \%), 8 q(25 \%)$ and $20 q(60 \%)$ and the most frequent losses were on $4 p(17.5 \%)$, $4 q(20 \%)$ and $18 \mathrm{q}(25 \%)$. The smallest region of copy number aberration was mapped to gain of $8 \mathrm{q} 24$ in five cases. The precised locations found in this study are quite relevant to previous reports. In addition, these results highlight several chromosomal regions that may harber important genes for CRC. A correlation analysis between the copy number aberration and the stage in our total material showed a statistically significant ( $p$ $\leq 0.05$ ). Increased copy number of chromosome arms $7 p, 12 q$ and $16 p(p=0.0389$ each) were significantly associated with Dukes' B and Dukes' $\mathrm{C}$, while increased copy number of chromosome arm 17q $(p=0.0471)$ was associated more with Dukes' A and Dukes' C. Our data suggesting that genes on those chromosomes may play key role in CRC progression and provide several starting points for the isolation of candidate oncogenes and tumor suppressor genes.

\section{PO:08:61}

Evaluation of CGH for the molecular diagnosis of NHL subtypes

M. Salehi ${ }^{1}$, R. Salehi ${ }^{1}$ and M. Behjati ${ }^{1}$
${ }^{1}$ Dept. of Genetics and Molecular Biology, Medical School, Isfahan University of Medical Sciences, Isfahan 81744-176, Iran

In order to define relationship between cytogenetic abnormalities and prognosis and the subtypes of the non-Hodgkin's lymphoma (NHL) patients, we employed the technique of Comparative Genomic in situ Hybridization (CGH). This technique allows, in a single, rapid analysis, the detection of chromosomal gains and losses in the complete tumor genome. It also requires only a small amount of tumor material and allows the detection of genetic abnormalities independent of the presence of metaphase spreads.

In this study, we investigated 33 lymph node biopsy samples from patients diagnosed with Bcell non-Hodgkin's lymphoma using control DNA, FITCE labeled, and tumor DNA, Texas red labeled, were co-hybridize to a normal metaphase spread. Images were acquired using an epifluorescence microscope and the commercially available image analysis system ISIS from MetaSystems. Ratio profiles of the fluorescence intensities of tumor DNA and control DNA were calculated for each individual chromosome.

Our results demonstrated that, using $\mathrm{CGH}$, $24 / 33(73 \%)$ of the patients exhibited chromosomal imbalances. Some chromosomal regions such as 3q22, 3q26, 8q24, Xq26, 2p23, 2p14 and $18 \mathrm{q} 21$ were frequently amplified while frequent deletions were found at some other chromosomal regions such as 5q11-13, 8p2, 9p2, 13q21-32, and $17 \mathrm{p}$.

In general it appeared that there might be a correlation between the total number of events (gains and losses) per tumor detected by $\mathrm{CGH}$ and aggressiveness of the tumor. Even though the exact target of the observed amplifications and deletions by CGH may not be known, but the total number of events detected by $\mathrm{CGH}$ might be considered as a prognostic marker.

\section{PO:08:62}

\section{A novel FISH assay for SS18-SSX fusion type in synovial sarcoma}

C. Surace ${ }^{1}$, F. Mertens ${ }^{1}$, I. Panagopoulos ${ }^{1}$, E. PQElsson ${ }^{1}$, M. Rocchi ${ }^{2}$ and N. Mandahl ${ }^{1}$ 
${ }^{1}$ Department of Clinical Genetics, Lund University Hospital, Lund, Sweden; ${ }^{2}$ DAPEG, Section of Genetics, University of Bari, Bari, Italy

Synovial sarcoma is a morphologically, clinically and genetically distinct entity that accounts for 5 to $10 \%$ of all soft tissue sarcomas. The majority of synovial sarcomas carries a $\mathrm{t}(\mathrm{X} ; 18)$ (p11.2;q11.2) chromosome translocation, that is a specific cytogenetic rearrangement, being present in more than $90 \%$ of the cases of synovial sarcoma and not in any other tumours. It produces three types of fusion gene formed in part by SS18 from chromosome 18 and by SSX1, SSX2, or, rarely, SSX4 from the X chromosome. It has been shown that in synovial sarcoma a clear correlation exists between type of fusion gene and clinical outcome. SS18-SSX fusion transcript can be detected by PCR amplification, requiring access to good quality RNA. In order to obtain an alternative tool to diagnose and follow this malignancy, we developed a fluorescence in situ hybridization (FISH) assay that could distinguish between the two most common fusion genes, i.e., SS18-SSX1 and SS18-SSX2, which cannot be discriminated using conventional chromosomal banding methods. The specificity of the selected BAC clones used in this FISH assay, as well as the sensitivity of the analysis in metaphase and interphase cells, was examined in a series of 22 synovial sarcoma samples without awareness of fusion gene status (previously determined by RTPCR). In all samples, the type of fusion gene was correctly identified by FISH, comparing with the results obtained by PCR. Thus, the assay described here should be useful for identifying fusion gene status in samples from which insufficient amounts of RNA could be extracted.

\section{PO:08:63}

Characterization of $4 q, 8 p, 13 q, 16 q$ and 17 in six hepatocellular carcinoma cell lines using region-specific multiplex-FISH probes

\author{
W. Tjia ${ }^{1}$, S. Sham ${ }^{1}$, L. Hu ${ }^{1}$ and X. Guan ${ }^{1}$ \\ ${ }^{1}$ Department of Clinical Oncology, University of \\ Hong Kong
}

Deletions in $4 q, 8 p, 13 q, 16 q$ and $17 p$ were frequently reported in hepatocellular carcinoma (HCC) by comparative genomic hybridization and loss of heterozygosity studies; yet, detailed chromosome structural aberrations are not known. We therefore characterize structural changes in these arms and identify the minimal deleted regions and breakpoints. By multiplex-color FISH (M-FISH) with chromosome region-specific probes (CRPs), chromosome structural aberrations of $4 \mathrm{q}, 8 \mathrm{p}, 13 \mathrm{q}, 16 \mathrm{q}$ and 17 , in six $\mathrm{HCC}$ cell lines, BEC7402, QGY7703, PLC8024, HepG2, H2M and H4M were studied. All CRPs, which were generated from microdissected DNA, were specific and strong in intensity, and sensitive enough to detect chromosome structural aberrations including translocation, deletion and amplification of target regions. Chromosome structural aberrations were matched with the results of CGH, yet additionally several cryptic breakpoints, especially those resulted from translocation. The findings by M-FISH using CRPs effectively narrowed down the position of breakpoints, making the further BAC screening more efficient. Chromosome structural aberrations were matched with the results of $\mathrm{CGH}$, yet additionally several cryptic breakpoints, especially those resulted from translocation. The findings by M-FISH using CRPs effectively narrowed down the position of breakpoints, making the further BAC screening more efficient. The breakpoints in $16 \mathrm{q} 22$ and $13 \mathrm{q} 14$ were finally mapped at $341 \mathrm{~K} 23$ and between $240 \mathrm{M} 20$ and 456B18, repectively.

\section{PO:08:64}

\section{A translocation $\mathbf{t}(3 ; 11)$ in an ovarian carcinoma cell-line inactivates the FHIT gene.}

\section{P. Willem Belot ${ }^{1}$ and N. Reckviashvili}

${ }^{1}$ Department of Haematology and Molecular Medicine, National Health Laboratory Services and the University of the Witwatersrand, Johannesburg, SA

The molecular events driving the pathogenesis of ovarian cancer have not been clearly elucidated. Genetic instability and the involvement of 
common fragile sites are being reported in an increasing number of human cancer studies. In particular the putative tumor suppressor gene FHIT (fragile histidine triad) which lies within the most common fragile site, FRA3B, has been shown to be involved in a variety of tumors including a subset of ovarian carcinoma. In most tumors the preferred mechanism of FHIT gene inactivation is through large intragenic deletions or promoter hypermethylation. Only four tumor associated translocations have been reported so far of which one was described in a breast cancer cell line.

We describe a translocation $\mathrm{t}(3 ; 11)(\mathrm{p} 14 ; \mathrm{p} 15)$ present in an early passage ovarian carcinoma cell line. Using fluorescence in-situ hybridisation (FISH) with selected YACs and BACs clones we mapped the breakpoint on chromosome 3 within the FRA3B loci. reverse transcriptase polymerase chain reaction (RT-PCR) further refined the breakpoints and showed that the translocation resulted in the complete loss of FHIT expression. This finding supports a role for FHIT in some ovarian carcinoma.

\section{Aneuploidy Symposium}

\section{L148}

\section{Recombination and aneuploidy in humans}

\section{T. Hassold ${ }^{1}$}

${ }^{1}$ Department of Genetics and Center for Human Genetics, Case Western Reserve University, Cleveland, Ohio 44106-4955, USA

Despite the clinical importance of human aneuploidy, we have been ignorant of the causes of meiotic nondisjunction, the process that gives rise to trisomic progeny. Over the past decade, however, genetic mapping studies have led to the identification of the first molecular correlate of human nondisjunction; i.e., altered levels and positioning of recombinational events. These observations have led to the idea that human meiotic nondisjunction may involve 'two hits': first, the establishment in prophase of a 'vulnerable' bivalent and second, abnormal processing of the bivalent at metaphase I or II. The pre- sentation will summarize data on trisomies 16,18 and sex chromosome trisomies as they relate to this model.

Additionally, we will summarize cytological studies designed to build chromosome-specific human genetic maps. These studies rely on recently developed immunofluorescence methodology; specifically, immunostaining of pachytene preparations using antibodies against MLH1 (a mismatch repair protein thought to localize to meiotic recombination nodules) and SCP3 (a component of the lateral element of the synaptonemal complex) makes it possible to visualize the sites of meiotic exchanges. Our initial analyses of several thousand pachytene stage cells from 50 males make it clear that this approach does, indeed, fulfill criteria predicted for a molecule that 'marks' the sites of exchanges. Thus, in subsequent studies we have used this approach to characterize the number and location of exchanges in a series of males, and to ask whether abnormalities in the recombination pathway are evident in infertile males. The results of these analyses will be presented at the symposium.

\section{L149}

\section{A model system for increased non-disjunction in older oocytes}

\section{S. Bickel ${ }^{1}$}

\author{
${ }^{1}$ Dartmouth College, Hanover, NH 03755 USA
}

As women age, the incidence of meiotic nondisjunction rises dramatically. Although increased maternal age has long been correlated with errors in chromosome segregation, the molecular defects that give rise to age-dependent meiotic nondisjunction in human oocytes are largely unknown. One major limitation has been lack of a suitable model system that recapitulates the higher levels of meiotic nondisjunction observed in older human oocytes. We have developed an experimental regimen using Drosophila melanogaster to study error prone segregation in older oocytes. Using a sensitized genetic background in which sister chromatid cohesion is compromised, we have shown that Drosophila oocytes exhibit a significant increase in meiotic nondisjunction following aging. I will describe these initial 
experiments and our continued analysis of segregation defects in older oocytes.

\section{L150}

The role of the meiosis-specific protein SCP3 in chromosome segregation and aneuploidy

\section{Höög ${ }^{1}$}

${ }^{1}$ Department of Cell and Molecular Biology, Karolinska Institutet, Stockholm, Sweden

Aneuploidy (trisomy or monosomy) is a leading genetic cause of pregnancy loss in humans and results from errors in meiotic chromosome segregation. We have developed a novel mouse model system where a single gene defect predisposes to aneuploidy (Yuan et al (2000) Molecular Cell 5, 73-83; Yuan et al (2002) Science, 296, 11151118). We show that absence of Synaptonemal Complex Protein 3 (SCP3) promotes aneuploidy in murine oocytes by inducing defective meiotic chromosome segregation. The abnormal oocyte karyotype is inherited by embryos, which die in utero at an early stage of development. In addition, aneuploidy and embryo death in SCP3deficient females increases with advancing maternal age. We find that SCP3 is required for the organization of the cohesin core, for the structural integrity of meiotic chromosomes and for chiasmata formation, suggesting that altered chromosomal structure triggers chromosomal non-disjunction. The SCP3-null mouse model system is used for studying the cause of aneuploidy in female germ cells, how such cells are (inefficiently) eliminated and the consequences of aneuploidy for germ cell and embryo development.

\section{L151}

\section{Examination of recombination patterns by maternal age among nondisjoined chromosomes 21 of maternal origin.}

S. Sherman ${ }^{1}$, E. Feingold ${ }^{2}$ and N. Lamb ${ }^{1}$

\author{
${ }^{1}$ Department of Human Genetics, Emory \\ University, Atlanta GA; ${ }^{2}$ Department of \\ Biostatistics, University of Pittsburgh, \\ USA
}

Advancing maternal age has long been identified as the primary risk factor for human chromosome trisomy. In addition, altered patterns of meiotic recombination have now been associated with an increased risk of nondisjunction. For trisomy 21, these 'susceptible' patterns include exchange near the centromere or single, telomeric exchanges. To date, however, no relationship has been found between these patterns on chromosome 21 and maternal age. As part of an ongoing study of maternal trisomy 21 (ts21), we examined the recombination status of 562 ts 21 cases. The cases were genotyped at STR markers spanning chromosome 21 and divided into groups based upon maternal age at the time of the trisomic birth $(<29,29-34,>34$ years of age $)$ and tetrad analysis used to infer the meiotic exchange patterns. Achiasmatic tetrads accounted for $39 \%, 21 \%$ and $28 \%$, respectively, of each age population. For tetrads with exchanges, the location of the exchanges varied between the groups in an age-dependent manner. Among the youngest group, $62 \%$ of nondisjoining oocytes had ãsusceptibleä exchange patterns. These patterns were less frequent in the mid (44\%) and older $(33 \%)$ age groups, which instead contained increased proportions of stable, medially located exchange. These data suggest that oocytes of younger women, with functional meiotic apparatus and/or robust ovarian environment, are able to properly resolve all but the most susceptible exchange patterns. As women age, however, meiotic mechanisms erode, making it difficult to resolve even stable exchange events.

\section{L152}

Is there a relationship between trisomy risk and rate of ovarian aging?

W. Robinson ${ }^{1}$, K. Bretherick ${ }^{1}$, C. Brown ${ }^{1}$, S. Watson ${ }^{2}$ and W. Lam ${ }^{2}$

${ }^{1}$ Dept. of Medical Genetics, U. of British

Columbia, Canada; ${ }^{2}$ Dept. of Pathology,

U. of British Columbia, Canada 
Most trisomies arise as consequence of abnormal chromosome segregation during oocyte maturation and occur more frequently with maternal age. Some data indicate that trisomy risk may be more closely related to ovarian age than maternal age. We thus hypothesize that genetic factors leading to premature loss of ovarian follicles should be increased in women experiencing a trisomic pregnancy at a relatively young age. Large sized alleles ( $>34$ repeats) at the FMR1 locus and X-chromosome rearrangements are two factors that have been associated with premature ovarian failure (POF). FMR-1 allele size was determined in 192 women who had a maternal meiotic nondisjunction event leading to a pregnancy with trisomy or uniparental disomy. The resulting distribution of allele sizes was similar to that found in 107 controls. Specifically, there was no excess of alleles $>34$ repeats overall $(4.7 \%$ of patient versus $5.1 \%$ of control alleles) or when subdivided by maternal age or type of abnormality. We previously reported an increase in skewed X-chromosome inactivation (XCI) among women experiencing a trisomic pregnancy. The possibility of a microdeletion or microduplication of the $\mathrm{X}$ as an explanation for skewing is currently being investigated in a subset of these women using a high-resolution $(\sim 300 \mathrm{~kb})$ genomic array. While FMR1 status and X-chromosome abnormalities may contribute little to overall risk of trisomy, it is still likely that there is some variability in risk of trisomy at a particular maternal age due to cumulative effects of common genetic variants affecting ovarian and chromosomal aging.

\section{L153}

Karyotype-specific failure of mouse ES cell differentiation in vivo and in vitro N. Takagi ${ }^{1}$, M. Shoji ${ }^{2}$ and T. Tada ${ }^{3}$

\author{
${ }^{1}$ Hokusei Gakuen University, Sapporo; \\ ${ }^{2}$ Youdosha, Tokyo; ${ }^{3}$ Inst. Frontier Med. Sci., \\ Kyoto University, Kyoto
}

Differentiation potential was studied in three mouse ES cell lines carrying an unbalanced form of Searle's translocation, T(X;16)16H. The unbalanced karyotype designated as 40,XY, - 16, $+\operatorname{der}(\mathrm{X}), \mathrm{t}(\mathrm{X} ; 16)$ mat is doubly monosomic for the proximal $50 \%$ segment of chromosome 16 and the distal $37 \%$ of the $\mathrm{X}$ chromosome. These cell lines were uniformly unable to form typical embryoid body failing in the formation of the outer endoderm layer. Cell aggregates they formed remained undifferentiated in suspension culture for more than two weeks, while karyotypically normal ES cell lines gave rise to some balloon-like cystic embryoid bodies by the 8th day of differentiation. These mutant cell lines produced much fewer and smaller tumors with a longer latent period after subcutaneous injection into nude mice than chromosomally normal ES cells. Unexpectedly, the degree of tissue differentiation in these tumors was comparable to those derived from karyotypically normal ES cells. A chromosome study showed, however, that the majority of tumor cells had lost the X16 [der(X)] chromosome and gained an additional copy of chromosome 16 . The present observation suggests that the monosomy for the proximal segment of chromosome 16 is mainly responsible for the failure of endoderm differentiation. It remains unknown, however, whether the generally low differentiation potential in these ES cell lines is attributed to the partial monosomy 16 , or non-inactivation of X16 chromosome, or both.

\section{L154}

Array-CGH in the search for tetralogy of Fallot genes on chromosome 8

\author{
M. Poot ${ }^{1}$, M. Eleveld ${ }^{1}$, M. Van Dam ${ }^{1}$, \\ R. Hochstenbach ${ }^{1}$ and J. Giltay ${ }^{1}$ \\ ${ }^{1}$ Department of Medical Genetics, University \\ Medical Centre Utrecht, P.O. Box 85090, 3508 \\ AB Utrecht, The Netherlands
}

After an uneventful pregnancy of 37 weeks a child with multiple dysmorphic features of head, hand and feet, and a Tetralogy of Fallot (ToF) was born. The mother had previously given birth to three healthy children. The karyotype of the child was initially defined as $46, \mathrm{XY}, \mathrm{i}(8)(\mathrm{q} 10)$. The mother proved to be carrier of a pericentric inversion with $8 \mathrm{p} 22$ and $8 \mathrm{q} 21.2$ as the breakpoints. DNA of the child was examined by array-CGH with an array containing 3,343 
FISH-localised BAC DNA probes, covering the entire genome with an average spacing of approximately $1 \mathrm{Mb}$ (Vissers et al. (2003) $A m J$ Hum Genet 73:1261-1270). This analysis revealed a $6.1 \mathrm{Mb}$ deletion of the distal part of $8 \mathrm{p}$ (8p23.1pter) and a $30.0 \mathrm{Mb}$ duplication of the distal part of $8 \mathrm{q}$ (8q21.3qter), as a result of recombination within the inverted segment.

In $55 \%$ of the patients with a del(8p23.1pter)dup(8q22.1qter); (OMIM 179613) conotruncal heart defects have been found. None of the 15 genes with known function in the deleted region of $8 p$ of our patient have been linked to ToF. In contrast, the duplicated region of chromosome $8 \mathrm{q}$ contained a zinc finger protein gene (ZFMP2; OMIM 603693; also known as FOG2). This gene may be a candidate gene for ToF, because point mutations have been associated with ToF. The ToF candidate gene GATA4 gene is not contained within the $8 \mathrm{p}$ deletion. We conclude that the improved resolution of array-CGH will help to identify chromosomal regions and candidate genes involved in the pathogenesis of ToF.

\section{L155}

\section{Etiology of recurrent trisomy}

\section{Warbuton}

Babies Hospital South, 3959 Broadway, Room 406, New York, NY 10032, USA

No abstract was submitted for this talk.

\section{PO:08:65}

\section{Effects of the herbicide atrazine on aneuploidy in Pacific oysters, Crassostrea gigas}

K. Bouilly ${ }^{1}$, A. Leit $^{1}$, H. Mccombie ${ }^{1}$, R. Chaves ${ }^{2}$, H. Guedes-Pinto ${ }^{2}$, P. Boudry ${ }^{1}$ and $S$. Lapague ${ }^{1}$

${ }^{1}$ IFREMER, Laboratoire de Génétique et Pathologie, 17390 La Tremblade, France;

${ }^{2}$ University of Trás-os-Montes and Alto Douro, Centre of Genetics and Biotechnology, 5001-911 Vila Real, Portugal
Hypodiploid aneuploid cells have regularly been reported in the Pacific oyster, Crassostrea gigas. A negative correlation between this phenomenon and growth as well as evidence for a genetic basis have been shown. Furthermore, non-random chromosome loss was also demonstrated in G-banded aneuploid karyotypes of $C$. gigas. Chromosome pairs 1, 5, 9, and 10 were characterised by the loss of one chromosome. The present study investigated the effects of the herbicide atrazine on the level of aneuploidy in this species. Firstly, $C$. gigas adults and juveniles were subjected to different atrazine doses $(10 \mu \mathrm{g} / 1$ representing a peak value found in a polluted environment and $100 \mu \mathrm{g} / \mathrm{l})$. A positive relationship between atrazine concentration and aneuploidy was observed. Moreover, the aneuploidy level of a sample of juveniles, previously exposed to atrazine, which were subsequently transferred to non polluted conditions, remained significantly different between treatments. Furthermore, a progeny of oysters exhibited significantly higher aneuploidy levels when their parents had previously been exposed to atrazine. Thus, the aneuploidy phenomenon persists in time both within and between generations. Additionally, restriction endonuclease banding was used on the progeny to identify which chromosomes were missing. The identity of chromosomes lost is not influenced by atrazine as the same chromosome pairs were affected by the loss of one chromosome. Further investigation is required to enable a better understanding of aneuploidy in oysters, especially as to why cells tolerate the loss of these chromosomes, and why some chromosomes are more easily lost than others.

\section{PO:08:66}

\section{The role of viruses (EBV, CMV, HPV etc) in reproduction and aneuoploidy}

\author{
V. Culic ${ }^{1}$ \\ ${ }^{1}$ Department of Medical Genetics and Genetic \\ Counseling Unit, Clinical Hospital, Split, Croatia
}

Each chromosome contains two centrioles which adhere to each other through the cell cycle and normally separate only once during the G1-to-S cell cycle transition, resulting in centrosome 
duplication. Centrosome duplication is regulated by many intracellular events that are essential in maintaining genomic stability. Abnormal centrosome duplication is tightly linked to aneuploidy. Spontaneous abortions appear with the incidence of $12-15 \%$ in population. In spontaneous abortions there are $25-60>\%$ of chromosome abnormalities, and in most cases there are triploidies, tetraploidies and polyploidies. The genes associated with cell cycle abnormalities are p53, Brca1, Brca2, Gadd45, human papillomavirus type E6 and E7 also leads to mitotic defects. The theory of 'two hits' for one unstable cell cycle resulting with aneuploidy is still in bases of these events. From the couples in the genetic counselling process with normal karyotype and aneuploidy in aborted material analysed by flow cytometry, we found CMV and EBV reactivation or new infection in both parents before and/or during pregnancy. We analysed those with aneuploidy in aborted material and significant serologic findings in both parents. A retrospective analysis was done over 500 couples with one or more spontaneous abortion and 296 paraffin embedded samples were found. 41 placentas was analysed by flow: 27 (66\%) diploid and 14 (34\%) aneuploiyd. From all number of 29088 (30\%) had IgM or/and high IgG for EBV and $12(4 \%)$ for CMV. HPV positive diploid paraffin embedded sample from one couple with three consecutive spontaneous abortion was found.

\section{PO:08:67}

\section{The Down syndrome frequency dynamics in Lviv region (Ukraine) for 1985-2001 period}

\section{Z. Fedoryshyn ${ }^{1}$, O. Hnateiko ${ }^{1}$ and D. Zastavna ${ }^{1}$}

${ }^{1}$ Institute of Hereditary Pathology of Academy of Medical Science of Ukraine

The Down syndrome average in Lviv region (1985-2001 period) composed 0,7 on 1000 newborns or 1:1430 newborns. Analysis of received results showed the increased frequency of Down syndrome during this period. The average index of Down syndrome frequency in 1995-2001 was in 1,4 times higher comparatively to average index in 1985-1994 according to Lviv regional medical-genetic center data. The Down syndrome is confirmed cytogenetic among 94,4\% ones those diagnosed by the phenotype createria in the maternity hospitals. The cytogenetic cheks have showed 95,2\% Trisomy 21, 3,0\% mosaicism for Trisomy 21, 1,8\% Robertson's translocation of all cases.

\section{PO:08:68}

\section{Phenotypic and molecular analysis in 90 girls with Turner's syndrome}

\author{
R. Fernandez ${ }^{1}$ and E. Pasaro ${ }^{1}$ \\ ${ }^{1}$ Department of Psychobiology, University of A \\ Coruna, Spain
}

We performed a phenotypic and molecular analysis of sex chromosome mosaicism in 90 Turner syndrome patients. The investigation was carried out in three phases: 1) Cytogenetic (G-banding and FISH); 2) Molecular (PCR and SRY gene sequencing); 3) Comparative analysis of the phenotype.

The application of classical alpha-satellite probes (CEP-X and CEP-Y), painting probes (WCP-X and WCP-Y), two subchromosomal painting libraries (SCPL116, SCPL102) covering the short and the long arm of the $\mathrm{X}$ chromosome, XIST and DXZ4 probes allowed us to find a second cell line (mosaicism) in $90 \%$ of the patients; only $10 \%$ of the patients were defined as $45, \mathrm{X}$ non-mosaic. The most frequent mosaic was $45, \mathrm{X} /$ $46, \mathrm{XX}$; the presence of isochromosomes or pseudo-isochromosomes comprised 25\%, fragments $5 \%$ and $\mathrm{Y}$ chromosome $4.4 \%$. The patients who had been previously diagnosed as mosaics displayed a higher complexity in their karyotypes due to the presence of new cell lines. Only one of the patients showed mental retardation due to a small ring der(X).

The Y chromosome and the SRY gene were present in blood and ovarian tissue in four patients. The analysis of the SRY sequence in one of the patients indicated the presence of two copies of the SRY gene, simulating a heterozygous state of the gene. One of the copies presented a point mutation Arg59Gly within the HMG-box. 
PO:08:69

\section{Demonstration of trisomy 18 in an autolytically altered fetus: a case report}

\author{
U. Gamerdinger ${ }^{1}$, D. May $^{1}$ and A. Seidel ${ }^{1}$ \\ ${ }^{1}$ Institute of Pathology, University of Gießen, \\ Germany
}

Prolonged time interval between intrauterine fetal death (IUFD) and abortion commonly leads to advanced autolytic alteration of the fetus. In such cases culture failure often impedes karyotype analysis. Here we present a case in which the combination of fetal pathology and molecular-cytogenetic methods was helpful in the evaluation of chromosome abnormality associated with fetal death.

A fetus aborted after IUFD at 18th gestational week showed advanced autolysis at outer inspection and also for the inner organs. External fetopathologic examination demonstrated developmental delay, hygroma colli, cranio-facial dysmorphias, anal atresia and ambiguous genitalia. The placenta was hypotrophic with abnormal villi and a single umbilical artery. The anomalies were suggestive of chromosome aberration. In order to prove suspected chromosomal aneuploidy molecular-cytogenetic studies were carried out.

Fetal DNA for CGH was extracted from tissue samples of kidney and lung. Despite DNA-degradation $\mathrm{CGH}$ revealed a gain of the entire chromosome 18. To verify the result an interphase-FISH with a probe for $18 \mathrm{cen}$ was performed on cryo-sections of the kidney. Most nuclei exhibited three signals but also several showed only two signals. The latter were interpreted as artifact due to truncated nuclei or reduced hybridization efficacy in the macerated tissue but a mosaic could not be excluded at least.

Postmortal diagnosis in fetal death is of importance in counseling patients regarding recurrence risk. We could demonstrate that despite autolyic alteration of a fetus which inhibits classical chromosome analysis molecular-cytogenetic methods may be helpful in unraveling aneuploidy.
PO:08:70

\section{Cytogenetic analysis chromosomal status of subjects from the regions in the vicinity of uranium contaminated areas}

\author{
D. Jovicic ${ }^{1}$, S. Milacic ${ }^{1}$ and R. Kovacevic ${ }^{1}$
}

${ }^{1}$ Institute of Occupational Health and Radiological Protection 'Dr Dragomir

Karajovic', Belgrade, Serbia and

Montenegro

The study was aimed at determining possible karyotype genotoxic effects in individuals from the regions close to the contaminated areas. Biological dosimetry was performed using modified Moorhead's micromethod. Our studies included the targeted group of 29 patients from the affected regions. The subjects were averagely aged $39.5+2.8$ years. Average age of the control group $(\mathrm{K})$, unexposed to the effects of the known genotoxic agents comprising 22 individuals was $28.3+1.2$ years. The presented data evidenced that increased incidence of the chromosomal aberrations was found in 6 subjects, accounting for $20.6 \%$. Dicentric type changes were evidenced, as well ring chromosomes and acentric fragments, which are, at the same time the most frequent aberrations. The changes are considered reparable aberrations accounting for $2-3 \%$ in metaphases of the unexposed individuals. Statistical data processing evidenced significant difference $(p<0,005)$ between structural chromosomal aberrations in the studied and control groups, as well as in the number of chromatid aberrations $(p<0.05)$. Based on the obtained data it may be concluded that human karyotype changes were present in the studied group, resulting from interaction of ionizing irradiation and other genotoxic agents, with possibility of potent synergistic effects. It is necessary to stress the importance of further monitoring and control of the general population health, particularly due to possible late genetic effects that may effect future generations. 


\section{PO:08:71}

\section{Chromosome imbalance causes elevated apoptosis in pre-mature neurons}

\author{
Y. Kai ${ }^{1}$, Y. Kazuki ${ }^{2}$, S. Abe ${ }^{1}$, C. Okita ${ }^{1}$ \\ and M. Oshimura ${ }^{1}$
}

${ }^{1}$ Department of Biomedical Science, Institute of Regenerative Medicine and Biofunction, Graduate School of Medical Science, Tottori University, Japan; ${ }^{2}$ Department of Molecular and Cell Genetics, Graduate School of Medical Science, Tottori University, Japan

At least $8 \%$ of all human conceptions have major chromosome abnormalities and the frequency of chromosomal syndromes in newborns is $>0.5 \%$. Chromosome abnormality syndromes show nonspecific phenotypic features. For example, most of the syndromes have a phenotype of mental retardation. Although these disorders make a large contribution to human morbidity and mortality, there are little data showing the molecular mechanisms.

Previously, we showed that ES cells containing an extra human chromosome 21 led to the elevated apoptosis in pre-mature neurons in vitro. To test a hypothesis that the chromosomal imbalance itself might stimulate the apoptotic discrimination, we made mouse ES cell lines with chromosome abnormalities by two methods, and investigated the apoptosis during neurogenesis. Firstly we used microcell-mediated chromosome transfer (MMCT) to create ES cells containing a human chromosome 6,11 or 21 , respectively. Secondly, we created ES cell lines with mouse chromosome aneuploidy by sub-cloning of normal ES cells. We investigated the apoptosis during neuronal differentiation (at day 3 ) in these chromosomal aberrant ES cell lines using the SDIA method. Accelerated apoptosis was observed in all ES cell lines with chromosomal imbalance compared to ES cells with normal karyotype. These data suggest that the increase in apoptosis at the early neural stem cell stage might be responsible for the reduction of brain neurons in chromosomal abnormality syndromes, which cause mental retardation. This in vitro differentiation system using chromosomal aberrant ES cells is a useful tool for identification of gene(s) responsible for recognition of chromosomal imbalance in mammalian cells.

\section{PO:08:72}

\section{Aneuploidy detection by FISH for postnatal, prenatal and preimplantation genetic diagnosis (PGD) in India}

\author{
P. Madon ${ }^{1}$, A. Athalye ${ }^{1}$, S. Dhumal ${ }^{1}$, \\ M. Kawle', V. Bandkar ${ }^{1}$, A. Sopariwala ${ }^{1}$, \\ N. Naik ${ }^{1}$, D. Naik ${ }^{1}$, S. Mohanty ${ }^{1}$ and \\ F. Parikh ${ }^{1}$
}

${ }^{1}$ Department of Assisted Reproduction and Genetics, Jaslok Hospital and Research Centre, Mumbai 400026, India

Fluorescence in-situ hybridization (FISH) has become a rapid and reliable genetic diagnostic tool in medicine. Jaslok Hospital and Research Centre is the first in India to routinely offer FISH on a wide range of tissues. We have studied over 1000 samples using the Vysis Aneuvysion probe kit, Metasystems software and a Zeiss Microscope. For PGD, we also use a combination of the PB probes for chromosomes 13, 16, 18,21 and 22 together with CEP $18, \mathrm{X}$ and $\mathrm{Y}$ in a 2-step procedure to detect aneuploidy of 7 chromosomes on single blastomeres biopsied from clevage-stage embryos obtained after ICSI. FISH on blastomeres using the Aneuvysion kit is more cost-effective. FISH on arrested embryos could detect chaotic embryos.

We have been able to detect $\mathrm{X}$ chromosome mosaicism for the first time in cumulus cells from follicular fluid obtained at pick-up of oocytes during IVF in a patient with low-grade mosaicism detected on karyotyping and confirmed by FISH on buccal smears. Cumulus cells can thus serve as an alternate source to detect mosaicism in women undergoing IVF.

FISH on sperm gives an insight into the percentage of hypo or hyperhaploid sperm. FISH on products of conception is routinely carried out to detect aneuploidies and triploidy. In Prenatal Diagnosis, a rapid report on uncultured amniocytes by FISH helps in allaying the anxiety of 
parents when the Triple Test shows a high risk. In cases from rural areas we detected Down syndrome by LSI FISH alone to make the tests affordable to the masses.

\section{PO:08:73}

\section{A comparative study on detection} efficiency of classical cytogenetic and interphase fluorescence in situ hybridisation in diagnosis of mosaic form of $\mathrm{X}$ chromosome aneuploidies

S. Mohaddes ${ }^{1}$, S. Tagavi ${ }^{1}$, J. Mohseni ${ }^{1}$, Z. Moosavi ${ }^{1}$, N. Bageri ${ }^{1}$ and H. Farahman

${ }^{1}$ Medical Genetic Unit, Faculty of Medicine, Tabriz university of Medical Sciences, Tabriz, Iran

Standard cytogenetics technique is of value in detection of numerical and structural chromosome abnormalities. However the detection of chromosomal mosaicism is often difficult due to time constrains and limited number of available metaphase cells for analysis. Interphase fluorescence in situ hybridisation (FISH) can be utilized to study the number of copies of a specific chromosome in interphase cells. The technique has valuable application in detection of chromosomal mosaicism since, a minimum of 100 cells can be analysed in a limited time.

We employed the GTG-banding assay followed by interphase FISH using DXZ1 (Q-Biogene) as probe to enumerate the $\mathrm{X}$ chromosome on peripheral blood samples of 88 women demonstrating the clinical features, compatible with $\mathrm{X}$ aneuploidies. Thirty-two samples were detected as 45,X and 3 samples as 47,XXX by GTG-Banding method and confirmed by Interphase FISH. Nine Samples were detected as suspicious 45,X/46,XX and 2 samples as $45, \mathrm{X} / 46, \mathrm{XX} / 47, \mathrm{XXX}$ by conventional cytogenetic studies and were subsequently confirmed by Interphase FISH. Five samples were diagnosed as normal by conventional cytogenetic studies, while 4 samples were shown to be mosaeic $45, \mathrm{X} / 46, \mathrm{XX}$ and the fifth sample 45,XX/46,XX/ 47, XXX by interphase FISH analysis. Six samples showed structural aberrations of chromosome X, which were undetectable by Interphase FISH. The remaining 21 samples were revealed to be normal by both methods.

The results indicate that interphase FISH is a useful and reliable tool in detection of mosaeic aneuploidies. However the technique has to be used as a parallel to conventional cytogenetic methods, which allows detection of structural chromosome abnormalities.

\section{PO:08:74}

\section{Spectrum of chromosome anomalies among the fetuses with an increased nuchal translucency}

\author{
L. Samoylova ${ }^{1}$, A. Latypov ${ }^{1}$, O. Shipacheva ${ }^{1}$ \\ and I. Boobis ${ }^{1}$ \\ ${ }^{1}$ Genetic Division of Tatarstan Republic Hospital, \\ Kazan, Russia
}

The aim of the study was to investigate the chromosome abnormality spectrum among the fetuses with an increased nuchal translucency in Tatarstan (Russia).

A total of 97 fetuses with an increased nuchal translucency at 11 to 14 weeks' gestation were evaluated cytogenetic prenatal diagnosis on chorion villi samples (CVS).

A chromosome anomalies was obtained in 46 fetuses $(47.4 \%)$. There are trisomy $21-11$ cases $(23,4 \%)$, monosomy $\mathrm{X}-8(17,4 \%)$, trisomy $18-7(15,2 \%)$, trisomy $13-8(17,4 \%)$. Another cases was marker chromosome $-8,+\mathrm{C}-4$ cases, trisomy $22-1$ case and 1 case with structural abnormality at 18 chromosome.

Measurement of nuchal translucency thickness improve the detection of any kind of chromosome trisomy in the first trimester of pregnancy. Although numerically limited, our experience confirms that increased nuchal translucency often observed in chromosomally abnormal fetuses.

\section{PO:08:75}

Two de novo partial trisomies $9 \mathrm{p}$ (p13-p24) characterized by CGH in two unrelated children
M. Santos ${ }^{1}$, C. Hernando ${ }^{1}$, A. Escalona ${ }^{1}$, M. Carrera ${ }^{2}$, G. Rodriguez-Criado ${ }^{3}$ and C. Fuster ${ }^{1}$ 
${ }^{1}$ Unidad de Biologia, Facultad de Medicina, Universidad Autonoma de Barcelona, E-08193 Bellaterra, Barcelona, Spain; ${ }^{2} \mathrm{CPC}$ Centro de Patologia Celular, Londres 6, Barcelona, Spain; ${ }^{3}$ Servicio de Genética Clinica, Instituto Hispalense de Pediatria, Sevilla, Spain

We report the use of comparative genomic hybrididization $(\mathrm{CGH})$ to define the extra chromosome material detected in the karyotype of two unrelated children. Both patients showed some typical clinical features from partial $9 p$ trisomy syndrome. Cytogenetic analyses of peripheral blood lymphocytes after Wright's G-banding revealed the presence of $13 p+$ and $22 p+$ chromosomes respectively. Parental karyotypes were normal in both. Partial 9p trisomy was disclosed by FISH analysis using multi-probe chromosomes analysis in one case and by 24-MulticolorFISH in the other. $\mathrm{CGH}$ studies revealed gains in 9p13.2-p24 and 9p13-p24 chromosome regions and at the same time discarted other genomic imbalances. These findings suggested that the differences between both phenotypes could be due to concomitant involvement of other chromosomes (chromosome 13 or 22) and maybe to mild differences in the size of trisomic segments involved. Our results, as previously reported cases which used methodologies other than the CGH (YAC DNA probes), support that 9p22 may be the critical region in some aspects of the trisomy $9 \mathrm{p}$ syndrome phenotype.

Acknowledgments: Financial support from SAF (2003-03894) and CIRIT (2001, SGR-00201).

\section{PO:08:76}

\section{A new patient with ring chromosome 22}

\section{A. Valetto ${ }^{1}$, V. Bertini ${ }^{1}$, R. Battini ${ }^{2}$,}

A. Battaglia ${ }^{2}$, G. Cioni ${ }^{2}$, E. Rapalini', F. Tinelli ${ }^{2}$ and P. Simi ${ }^{1}$

${ }^{1}$ Cytogenetic and Molecular Genetic Laboratory, S.Chiara Hospital, Pisa, Italy; ${ }^{2}$ Stella Maris Clinical Research Institute for Child and Adolescent Neuropsychiatry, Calambrone (Pisa), Italy.

The clinical phenotype of patients with ring chromosome 22 includes mental retardation with severe language impairment, hypotonia, and dysmorphic facial features. In recent years an increasing number of patients with microscopic as well as cryptic terminal deletion involving band 22q13 has been described and their phenotype shows clinical features overlapping with patients with ring chromosome 22. Loss of DNA in the 22q13.3 region may lead to a clinically recognizable syndrome named "22q13.3 deletion syndrome".

We report on a patient with a ring chromosome 22 who has hypotonia, profound mental retardation, language impairment, dysmorphic features and behavioral disorders.

To check if the critical region responsible of $322 \mathrm{q} 13.3$ deletion syndrome was absent in this ring, a FISH analysis using a probe corresponding to the ARSA locus has been performed; ARSA resulted deleted. A more detailed analysis of the deletion extent then was performed using a panel of fluorescent probes located within 22q13. These experiments allowed the identification of the breakpoint between CTA-299D3 and RP5925J7 probe, located in 22q13.32. Deletion extent could be estimated to be about $2.5 \mathrm{Mb}$, and this larger deletion may explain the severity of clinical features observed in our patient.

We would suggest considering this deletion in all children presenting with developmental delay/ mental retardation, absent or severely delayed speech, autistic-like behavioral disorders, and minor dysmorphic features. High-resolution chromosome analysis should be performed together with FISH or molecular studies to exclude this deletion syndrome.

\section{PO:08:77}

\section{A case with partial trisomy $7 p$ and monosomy 9p}

S. Yilmaz ${ }^{1}$, Y. Tarkan-Argüden ${ }^{1}$, A. Cirakoglu' ${ }^{1}$ A. Deviren ${ }^{1}$, D. Kuru', G. Guven ${ }^{1}$, E. Yosunkaya-Fenerci ${ }^{1}$, H. Kurt ${ }^{1}$, A. Yuksel ${ }^{1}$ and S. Hacihanefioglu ${ }^{1}$

${ }^{1}$ Dept. of Medical Biology, Cerrahpasa Medical School, Istanbul University, 34300, Istanbul, Turkey

This eleven months old boy, born to healthy, non-consanguinous parents referred to us for 
developmental delay and multiple congenital anomalies. The family history revealed that the parents had four first trimestr spontaneous abortuses, three girls who died during neonatal period from unknown etiology and two healthy daughters still alive.

Our case was born at term in normal centiles. In the prenatal ultrasonographic examinations, left kidney showed multiple cysts and in the postnatal period nephrectomy was done for renal complications. The physical examination revealed developmental delay, trigonocephaly, scarce scalp hair, hypotrichosis, narrowing of the bitemporal diameter, low-set dysmorphic ears, upslanting palpebral fissures, epikanthal folds, depressed nasal root, high arched palate, micrognati, small mouth, downturned corners of the mouth, congenital cardiac anomalies, genital anomalies, first and third toes overlapping the second on the right foot.

Cytogenetic analysis done with GTL banding to peripheral blood culture revealed add(9)(p24). The cytogenetic analysis of the parents showed that the father's karyotype was $46, \mathrm{XY}$ and the mother's was $46, X X$,der(9)t(7;9)(p21;p24). The brother and two sisters were also examined and in one of the sisters and the brother normal karyotypes were found while the elder sister appeared as balanced translocation carrier like her mother. Therefore, our proband's karyotype was 46,XY, der(9)t(7;9)(p21;p24)mat. FISH analysis using whole chromosome painting probes for chromosomes 7 and 9 was performed to identify balanced and unbalanced translocations.

Literature examination showed that the physical findings of the present case shows extensive overlap with both the findings of partial $7 p$ trisomy syndrome and partial $9 p$ monosomy syndrome.

\section{New Methodologies Symposium}

\section{L156}

\section{Application of scanning near field} optical/atomic force microscopy to the observation of human metaphase chromosomes.
${ }^{1}$ Division of Microscopic Anatomy and Bio-imaging, Nigata University Graduate School of Medical and Dental Sciences, Nigata, Japan

The present study aimed to introduce a method for observing immunostained chromosomes by a combination technique of atomic force microscopy (AFM) and scanning near field optical microscopy (SNOM). For this purpose, we designed an AFM combined with SNOM (i.e. SNOM/AFM), which can collect both topographical and optical images of the same portion of the samples simultaneously. We showed SNOM/AFM images of human chromosomes, which were immunostained with an anti-BrdU antibody and Alexa Fluor 488 after incorporation of BrdU into DNA. SNOM/AFM enabled the collection of fluorescence images of differentially immunostained chromatid simultaneously with topographical images of the same portion. The fluorescent images clearly showed the portion of sister chromatid exchanges (SCEs), and the corresponding topographical images provided precise information on the fine structure of those portions. We then observed by SNOM/AFM the human metaphase chromosomes immunostained with an anti-topoisomerase II antibody. By this technique, topoisomerase II immunoreactivity in the core of the chromosome arms and centromere was analyzable precisely in correlation of fluorescent images with the corresponding topographical images. Since the spatial resolution of the obtained fluorescent images was obviously higher than those by conventional fluorescence microscopy, SNOM/AFM is expected to serve as a tool for future studies of the structure of chromosomes in relation to the localization of their components.

\section{L157}

\section{Combined analysis of morphology and FISH for increased accuracy of cancer diagnosis}

M. Daniely ${ }^{1}$, L. Trakhtenbrot ${ }^{2}$, R. Rona ${ }^{3}$, G. Rechavi ${ }^{2}$, S. Law $^{3}$, A. Guber ${ }^{4}$, N. Amariglio ${ }^{2}$, I. Leibovitch ${ }^{5}$, T. Kaplan ${ }^{1}$ and $M$. Reichart ${ }^{1}$ 
${ }^{1}$ BioView Ltd, Rehovot, Israel; ${ }^{2}$ Dept. of Pediatric Hemato-Oncology and Inst of Hematology, The Chaim Sheba Medical Center, Tel-Hashomer, and Sacler School of Medicine, Tel-Aviv University, Tel-Aviv, Israel; ${ }^{3}$ Cytology Unit, Sapir Medical Center, Kfar-Saba, Israel; ${ }^{4}$ Dept. of Pulmonary, Sapir Medical Center, Kfar-Saba, Israel; ${ }^{5}$ Dept. of Urology, Sapir Medical Center, Kfar-Saba, Israel

Fluorescence in situ hybridization (FISH) is a valuable tool in clinical practice of cancer. However, high false positive and false negative rates complicate the interpretation of results. Combined analysis of morphology and FISH may enhance the accuracy of diagnosis. The Duet scanning system (BioView Ltd, Rehovot, Israel) enables combined analysis of several parameters in each cell by performing multiple scans of the same slide under various stains. This approach was applied to blood and bone marrow samples for the detection of various hematological malignancies as well as to urine and sputum samples for the detection of bladder and lung cancer, respectively. 500 blood/bone marrow samples, 35 urine samples and 8 sputum samples were analyzed. The preparation of samples included Giemsa/IHC staining followed by stain removal and FISH for chromosomal aberrations detection.

In the hematological samples, the combined analysis allowed (a) More accurate follow-up of MRD and bone marrow/stem cells transplantation by determination of lineage, clonality and maturity of cells carrying chromosomal rearrangement (b) Enhancing FISH accuracy when using probes with high false positive rate.

In urine samples the combined analysis detected as much as $100 \%$ of bladder cancer cases compared to only 60 and $80 \%$ detection rate reported by cytology and FISH, respectively. In sputum samples, a correlation was found between atypical morphology and chromosomal changes.

Overall, the combined analysis enabled increased specificity and sensitivity of cancer cells detection. These preliminary results indicate the advantage of using such approach in diagnosis of diverse cancer diseases.
L158

The ACE System: engineering artificial chromosomes to rapidly generate high-expressing cell lines for manufacture of recombinant proteins

M. Lindenbaum ${ }^{1}$, E. Perkins ${ }^{1}$, E. Csonka ${ }^{2}$, A. Greene' ${ }^{1}$, E. Fleming ${ }^{1}$, G. Hadlaczky ${ }^{2}$, N. Macdonald ${ }^{1}$, A. Maxwell ${ }^{1}$, C. Perez ${ }^{1}$ and H. Ledebur, jr. ${ }^{1}$

${ }^{1}$ Chromos Molecular Systems, Inc., Burnaby, BC Canada V5A 1W9; ${ }^{2}$ Institute of Genetics,

Biological Research Center, Hungarian

Academy of Science, Szeged,

Hungary

Mammalian artificial chromosomes are ideal for introducing large genetic payloads into cells in an autonomously replicating, non-integrating format. We have developed a unique satellite DNA-based Artificial Chromosome Expression (ACE) system. A platform architecture with multiple targeting sites allows transfer of single or multiple copies of genes into cells for use in recombinant protein expression, generation of transgenic animals and gene therapy. ACEs can be reproducibly generated de novo in different cell lines, readily purified from the host cellsâ chromosomes by flow cytometry and re-introduced into a variety of recipient cells where they are stably maintained for extended periods without selection. Platform ACEs form part of a biological engineering system including expression-optimized shuttle vectors to specifically transfer genes, and a plasmid-encoded proprietary integrase to catalyze specific incorporation of shuttle payload onto the ACE. Since genes of interest are introduced into a consistent genomic environment that supports high-level expression, relatively few clones need be screened to identify high expressers, leading to rapid, reproducible and efficient cell line generation. Cell lines expressing industry-relevant levels of recombinant protein $(>30 \mathrm{pg} /$ cell/day) can be generated in less than 12 weeks; amplification is unnecessary. Multiple rounds of loading can be carried out onto previously loaded ACEs, which allows incorporation of supplementary genes to 
improve protein quality or cell growth characteristics. By transferring ACEs to different cell lines the optimum producer for a particular product can be identified. The ACE System advantages of speed, efficiency and versatility provide an attractive alternative to conventional methods of cell line generation.

\section{L159}

\section{Identification of a complex chromosome rearrangement by cytogenetic, FISH, and genome-wide DNA array CGH analysis}

\author{
N. De Leeuw ${ }^{1}$, E. Bongers ${ }^{1}$, H. Mieloo ${ }^{1}$, \\ J. Willemen ${ }^{1}$, J. Veltman ${ }^{1}$ and \\ C. Van Ravenswaaij ${ }^{1}$ \\ ${ }^{1}$ Department of Human Genetics, University \\ Medical Centre, Nijmegen, The Netherlands
}

A 3-year old girl was referred to our centre for DNA array-based Comparative Genomic Hybridisation (array CGH) analysis because of preand postnatal growth retardation, microcephaly, cleft palate, an atrium septum defect, ataxia, hypotonia, psychomotor retardation and expressive aphasia. An apparently balanced, de novo translocation $\mathrm{t}(6 ; 9)(\mathrm{p} 21.3 ; \mathrm{q} 22.3)$ had been identified elsewhere.

Routine cytogenetic analysis was repeated, and in addition to the $t(6 ; 9)$, we found an interstitial deletion of the long arm of chromosome 13 (del(13)(q?21q31)). Subsequent Fluorescence in situ Hybridisation (FISH) analysis with whole chromosome paints of chromosomes 6,9, and 13, respectively, showed that chromosome 13 material was inserted into the derivative chromosome 6 at the translocation breakpoint. The question remained whether the chromosome rearrangement was balanced or not. Therefore, array CGH analysis was performed using a genome wide array of 3,500 BAC clones, evenly spaced over the human genome with an average resolution of $1 \mathrm{Mb}$. Array CGH analysis revealed a significant, net imbalance due to a deletion of approximately $7 \mathrm{Mb}$ (9 clones) between 13q21.33 and 13q22.3. The clinical features of our patient correspond well with those of individuals with a partial monosomy of this $13 \mathrm{q}$ region described in the literature.

DNA array $\mathrm{CGH}$ analysis appears to be an invaluable tool for diagnostic purposes, which, in combination with traditional cytogenetic and FISH analyses, will help us to unravel and identify subtle imbalances that otherwise would remain undetected.

\section{L160}

\section{A new approach to investigating transcription on lampbrush chromosomes}

\author{
A. Saifitdinova ${ }^{1}$, T. Kulikova ${ }^{1}$ and \\ E. Gaginskaya ${ }^{1}$
}

${ }^{1}$ Biological Research Institute of Saint-Petersburg University, 198504, Russia

It is really interesting to investigate sequences of RNA-transcripts from oocyte nuclei because they include sequences that are never transcribed in somatic cells. We have developed a new approach to investigation transcription on lampbrush chromosomes. Nuclear RNAs were isolated immediately from the manual dissected bird oocyte nuclei and treated with RNase free DNase I. RNA quality was tested using FISH of labeled cDNA samples with lampbrush chromosomes preparations. The results of FISH were the same as results of reverse transcription RNA labeling in situ. Reverse transcription PCR-analysis with specific primers used for RNA samples investigation. Reactions without Revertase were DNA presence controls. Transcription of chicken Z-macrosatellite and Ssp1 sequences and chaffinch centromeric sequence FCP was shown, whereas chicken WEcoRI and WXhoI repetitive DNAs are not transcribed. The results were confirmed by reverse transcription RNA labeling in situ with specific primers on lampbrush chromosome preparations. Quantitative analysis of known RNA transcripts could be produced by real time reverse transcription PCR-analysis with specific primers. This approach may be useful for making a cDNA library of oocyte nuclei transcripts. 
This work was supported by Russian Foundation for Basic Research (02-04-49116), Russian Ministry of Education (PD02-1,4-291) and Science (MK-2655.2003.04).

\section{L161}

\section{COMBO-FISH: Specific labeling of} genes and breakpoint regions by computer selected oligo-probe combinations

M. Hausmann ${ }^{1}$, J. Finsterle ${ }^{2}$, G. Hildenbrand ${ }^{2}$, E. Schmitt ${ }^{3}$, C. Großmann ${ }^{2}$, A. Rapp ${ }^{4}$, S. Stein ${ }^{2}$, M. Werner ${ }^{1}$ and C. Cremer ${ }^{2}$

${ }^{1}$ Institute of Pathology, University Hospital, Albertstr. 19, D-79104 Freiburg, Germany; ${ }^{2}$ Kirchhoff-Institute of Physics, University of

Heidelberg, Im Neuenheimer Feld 227, D-69120 Heidelberg, Germany; ${ }^{3}$ Dept. Biocomputing, Institute of Molecular Biotechnology e.V., P.O. Box 100 813, D-07708 Jena, Germany;

${ }^{4}$ Dept. Single Cell and Single Molecule

Techniques, Institute of Molecular Biotechnology e.V., P.O. Box 100 813, D-07708 Jena,

Germany

The principle of fluorescence in-situ hybridization (FISH) with COMBinatorial Oligo (COMBO) probes is presented as a new approach that permits specific labeling of any given genomic sites. COMBO-FISH takes advantage of homopurine/homopyrimidine oligo-nucleotides that form double strands as well as triple helices with intact genomic DNA. The latter can be performed without the need for prior thermal denaturation of the target sequence. An analysis of the human genome data bases has shown that homopurine/homopyrimidine sequences longer than 14 DNA bases are nearly homogeneously distributed over the genome and that they represent about $1-2 \%$ of the entire genome. Considering that the minimum observation volume in a microscope equipped with a high numerical aperture lens corresponds on average to a $\sim 250 \mathrm{~kb}$ chromatin domain, a set of distinct, uniformly labeled oligo-nucleotide hybridization probes can be configurated from human genome data base. This set is expected to exclusively co-localize within a $250 \mathrm{~kb}$ chromatin domain, although some of the oligonucleotides have additional binding sites somewhere else in the genome. Due to the diffraction limited resolution of a microscope, the fluorescence signals of the joined oligo probe set merges into a typical, nearly homogeneous FISH spot. Typical sets are introduced for tumor correlated genome loci. Experiments were performed as a very first proof of principle of COMBO-FISH. The technique was applied to human peripheral blood lymphocytes, formalin-fixed and paraffin embedded tissue sections, and routine bone marrow smears.

\section{L162}

\section{Analysis of small tissue samples by CGH on Arrays}

\section{P. Lichter}

Deutsches Krebsforschungszentrum, Im Neuenheimer Feld 280, 69120 Heidelberg, Germany

No abstract was submitted for this talk.

\section{L163}

Tissue microarrays for high-throughput early target validation

\section{G. Sauter}

Institute of Pathology, University of Basel, Schoenbeinstrasse 40, CH-4031 Basel, Switzerland.

No abstract was submitted for this talk.

\section{L164}

\section{Bioinformatics}

\section{A. Ladurner}

EMBL Heidelberg, Meyerhofstrasse 1, D-69117 Heidelberg, Germany

No abstract was submitted for this talk. 
L165

\section{ChIP on Chip}

\section{Roland Eils}

No abstract was submitted for this talk.

\section{PO:08:78}

\section{Expression of a yeast enhancing HAL2 gene for salt stress tolerance in eucalypt and the localization was detected using FISH}

\author{
S. Apisitwanich ${ }^{1}$, N. Thanananta ${ }^{1}$, \\ S. Siripatanadilok ${ }^{2}$, S. Peyachoknagul ${ }^{1}$ and \\ S. Suputtitada ${ }^{1}$ \\ ${ }^{1}$ Department of Genetics, Faculty of Science, \\ Kasetsart University, Bangkok, 10900; \\ ${ }^{2}$ Department of Forest Biology, Faculty of \\ Forestry, Kasetsart University, Bangkok, \\ 10900
}

The halotolerant gene, HAL2 of Saccharomyces cerevisiae Montache was cloned and constructed as the pBHATKU plasmid. This plasmid was transformed into the axillary buds of a eucalypt hybrids (Eucalyptus camaldulensis $\mathrm{x} E$. tereticornis) using Agrobacteriu $m$ mediated transformation. Eighteen percent of the tested explants using PCR gave a positive HAL2 fragment. Thirty one percent of those transformants (11 out of 35) could grow on MS6 medium supplemented with $300 \mathrm{mM} \mathrm{NaCl}$ but the control parents and their hybrid could not. The expression of the HAL2 gene in transgenic eucalypt hybrids in high salt condition $(300 \mathrm{mM} \mathrm{NaCl})$ was confirmed by RT-PCR. The location of the inserted HAL2 gene on chromosome was determined by fluorescence in situ hybridization (FISH). Some were localized near centromere and some were at terminal of the chromosome. Ten clones showed single positive signal consistently at the same position in all cells except one clone having single signal at the different position in different cell indicating that this clone was mosaic. These results indicated that Agrobacterium-mediated gene, HAL2 could integrate into many sites and distribute in different parts of the chromosome. All of the transgenic eucalypt hybrids were hemizygous genotype.

\section{PO:08:80}

\section{Physical nature of the chromatin dynamics: Higher order genome structure is flexible independently of chromatin proteins in Schizosaccharomyces pombe}

T. Kobori ${ }^{1}$, S. Sugiyama ${ }^{2}$, K. Takeyasu ${ }^{3}$ and T. Ohtani ${ }^{2}$

${ }^{1}$ National Food Research Institute \& Kyoto University; ${ }^{2}$ National Food Research Institute; ${ }^{3}$ Kyoto University, Japan

One of the structural bases for nuclear functions is a higher-order structure of chromatin that is expected to be flexible. Here we show one aspect of such flexibilities that depends exclusively on the physical nature of chromatin fibers by employing a nano-scale imaging technique, atomic force microscopy (AFM).

AFM revealed that Schizosaccharomyces pombe had a chromatin hierarchy as observed in other eukaryotes, including the particles with $45 \mathrm{~nm}$ in diameter that form a $350 \mathrm{~nm}$ fiber. An exposure of the chromatin to the high-salt solution (e.g., 1$2 \mathrm{M} \mathrm{NaCl}$ ) extracts a complete set of chromatin proteins including histones. In $S$. pombe, however, biochemical analyses showed that a brief exposure $(\sim 10 \mathrm{~min})$ of chromatin to high-salt solution did not realize any release of the chromatin proteins. Under this condition, AFM demonstrated that a stepwise structural transition took place during a brief exposure to $400 \mathrm{mM} \mathrm{NaCl}$; i.e., the $45 \mathrm{~nm}$ particles no longer existed, and, instead, a newly formed structure, $110 \mathrm{~nm}$ beads, was stably maintained regardless of a further increment of the salt concentrations. This process was reversible, and the $45 \mathrm{~nm}$ particles were reformed again by removing the salt from the $110 \mathrm{~nm}$ beads. This structural reversibility without any changes in the protein composition suggests the importance of the physical properties of chromatin-environment interactions. A chromatin-transition model can be proposed that predicts an ionsolvation in the transition. Careful 
observation of the time-dependent unfolding pathway could give an insight into a way to establish chromatin architecture.

\section{PO:08:81}

\section{Disordered three-dimensional structure of FISH treated chromosomes: visual analyses by FISH and atomic force microscopy}

\author{
M. Shichiri ${ }^{1}$, D. Fukushi ${ }^{2}$, S. Sugiyama ${ }^{1}$, \\ T. Yoshino ${ }^{1}$ and T. Ohtani ${ }^{1}$ \\ ${ }^{1}$ National Food Research Institute, Japan; \\ ${ }^{2}$ Niigata University, Japan
}

In molecular genetic studies, the fluorescence in situ hybridization (FISH) technique has been widely used for DNA mapping. Although the FISH treatments should cause structural changes and/or damage of the chromosomes, the influences of FISH treatment on the chromosome morphology are not well understood. We investigated the morphologic changes of the barley chromosomes during standard FISH processes using an atomic force microscope (AFM) that generates high-resolution topographic images of the samples. The chromosomes were observed in the selected four steps in which the samples were able to dry. The chromosomes collapsed by each step of the process and finally their heights decreased to one fourth of original height. To further analyze the chromosome damages, we performed multicolor FISH using human chromosomes and two or three probes targeting the genes only short distance apart $(0.7-1.5 \mathrm{Mb})$. The observed dispositions of the FISH signals were not always same but rather changeable among the samples. These results indicate that the chromosomes are severely damaged and lose the exact nature of their three-dimensional structure after standard FISH treatments. To avoid the destruction of the chromosome structures, we examined the effect of the re-fixation of the chromosome with aldehyde fixatives before FISH treatment. The preliminary experiment showed that the re-fixed chromosomes had an ability to keep its struc- ture against FISH treatments. The disposition of the FISH signals on the re-fixed chromosomes and the potential of this method toward much precious gene mapping and analysis of higher order chromosome structure will be discussed.

\section{PO:08:82}

\section{Usefulness of calyculin A for the diagnosis of trisomy 18 in AF culture}

\author{
M. Srebniak ${ }^{1}$, A. Wawrzkiewicz ${ }^{1}$, \\ W. Kazmierczak ${ }^{1}$, A. Wiczkowski ${ }^{2}$ and \\ A. Olejek ${ }^{1}$
}

${ }^{1}$ Silesian Medical Academy, Department and Clinic of Perinatology and Gynaecology, Zabrze, Poland; ${ }^{2}$ Silesian Medical Academy, Department of General Medical Biology, Zabrze, Poland

Calyculin A, an inhibitor of protein phosphatases type 1 and type 2A-serine/threonine, has proved to be useful in human karyotype examination. The addition of Calyculin A to in vitro cultures leads to premature chromosome condensation (PCC). All phases of the cell cycle (G1, S, G2, M) can be observed on one slide (Gotoh et al. 1995). We have previously reported that Calyculin A treatment does not influence chromosome banding patterns (Srebniak et al. 2003) and banded chromosomes can be analysed from a lymphocyte culture.

The aim of our work was to investigate whether it is possible to detect chromosome aberrations in cultures treated with Calyculin A.

Amniotic fluid culture previously diagnosed by a conventional karyotyping (Colcemid treatment) was used for that study. Additional cultures were subcultivated and treated with $100 \mathrm{nM}$ Calyculin A for $45 \mathrm{~min}$.

The karyotype was $47, \mathrm{XX},+18$ and it could be diagnosed from the culture treated with Colcemid. The GTG banding resolution occurred to be not satisfactory on preparations from the culture treated with Calyculin A, but trisomy was clearly seen (47 chromosomes). The aneuploidy in this sample could be easily detected, chromosomes were easy to count, but the GTG banding didnât allow identifying particular chromosomes. 
PO:08:83

\section{Development and evaluation of a computer-based tutorial to teach karyotyping in undergraduate practical classes and to assist in the screening for hypoprolificacy in boars}

J. Stephenson' ${ }^{1}$, N. Gibbons ${ }^{1}$, W. Morris ${ }^{1}$, A. Mileham ${ }^{2}$ and D. Griffin ${ }^{1}$

${ }^{1}$ Cell and Chromosome Biology Group, Brunel University, UK; ${ }^{2}$ Sygen International, Berkeley, CA, USA

The ability to karyotype human G-banded chromosomes is an essential skill for chromosome biologists and forms an integral part of the curriculum in many biological degrees. Karyotyping usually is taught by providing students with a photograph of G-banded chromosomes and scissors. This has the disadvantage that excessive time is taken cutting and pasting and comparatively little in learning pattern recognition. Here we report the development and evaluation of a computer-based student practical class 'KaryoLab'. Opinion analysis suggests that students greatly prefer the computer-based approach, we have evidence that marks are not compromised by using it and that exercises can be done in significantly shorter amounts of time.

It is thought that $\sim 10 \%$ of boars are subject to reduced fertility and cytogenetic studies suggest that approximately $50 \%$ of these have a recognisable chromosomal translocation. It is thus essential to ensure all boars are free of translocations before embarking upon an insemination programme as this could potentially save the pig breeding industry many millions of pounds in lost revenues from unproductive animals. G-band analysis is often thought to require highly skilled individuals however, in this study, we have adapted human 'KaryoLab' to instruct users in the skill of porcine karyotyping. Initial tests suggest that the tutorial that can be learned in the users' own time and improves the karyotyping skills dramatically. Indeed, users spotted the presence of an abnormality in over $80 \%$ of cases. We propose that this programme could be used for the screening of AI boars prior to artificial insemination.

Haematological Malignancy Symposium

\section{L166 \\ Clinical relevance of genomic aberrations in lymphomas}

\section{Bentz}

Medizinische Universitaetsklinik, Abt. Fuer Innere Medizin III, Robert-Koch Str. 8, 89081 Ulm, Germany

No abstract was submitted for this talk.

L167

\section{Chromosomal translocations involving the immunoglobulin genes in B-cell malignancies}

M. Dyer ${ }^{1}$

${ }^{1}$ Cancer Studies and Molecular Medicine, Department of Pathology, University of Leicester, Leicester LE1 7RH, UK

No abstract was submitted for ths talk.

\section{L168}

\section{Peculiarities of nonrandom aneuploidy patterns in acute lymphoblastic leukemia (ALL) \\ O. Haas $^{1}$}

${ }^{1}$ CCRI, Vienna, Austria

The simplest classification of childhood B-cell precursor ALL is according to chromosome number and ploidy level. The specific features of the aneuploid categories are the nonrandom numerical changes and the paucity of structural rearrangements. Near-haploid near-triploid and near-tetraploid cases are rare. Near-haploid clones usually coexist with hyperdiploid ones. They contain at least a haploid chromosome set with two copies of the sex chromosomes as well as of 10, 14, 18 and 21. The most common and prognostically favorable hyperdiploid subgroup is characterized by nonrandom trisomies of 4,6 , 
$10,14,17,18,20, \mathrm{X}$ and tetrasomy 21 . Four possible mechanisms could account for the formation of such karyotypes: the doubling of a nearhaploid set, an initial tetraploidization with subsequent losses or the sequential or simultaneous gain of chromosomes. The currently available data indicate that the last option is the default pathway, whereas the duplication of a near-haploid karyotype is another option. Also the acquisition order of chromosome is nonrandom: 21 and $\mathrm{X}$ are present in virtually all cases, followed by gains of $14,6,4,18,17,10,8,5,12$ and 11 . Nevertheless, almost $70 \%$ of the over-expressed genes in hyperdiploid ALL are located on chromosomes $\mathrm{X}$ and 21. Of particular interest is also the unique in vitro behavior of hyperdiploid leukemia. Since they rapidly undergo apoptosis, it is virtually impossible to propagate them in culture. It therefore also comes of no surprise that only a single cell line has been established so far from such a hyperdiploid ALL.

\section{L169}

\section{Gene expression profiling identifies prognostically relevant subgroups in adult acute myeloid leukemia}

\author{
L. Bullinger ${ }^{1}$, K. Dähner ${ }^{2}$, E. Bair ${ }^{1}$, \\ S. Frähling ${ }^{2}$, R. Schlenk ${ }^{2}$, R. Tibshirani ${ }^{1}$, \\ H. Dähner ${ }^{2}$ and J. Pollack ${ }^{1}$ \\ ${ }^{1}$ Stanford University, Stanford, California, USA; \\ ${ }^{2}$ University of Ulm, Ulm, Germany
}

In acute myeloid leukemia (AML), recurrent cytogenetic aberrations are used to classify patients for appropriate therapy. However, the current classification system does not fully reflect the heterogeneity of the disease, and treatment stratification is difficult, especially for intermediate-risk cases with normal karyotype. To further characterize adult AML at the molecular level, we profiled gene expression in 116 samples (including 45 with normal karyotype) using cDNA microarrays representing 26,260 genes. Unsupervised and supervised analysis readily identified gene-expression signatures characterizing known cytogenetic subgroups, and suggested numerous genes with potential pathogenic relevance. Further, unsupervised hierarchical clustering identified addi- tional subtypes, including clinically-relevant subgroups within the class of AML with normal karyotype. Using a randomly-selected training set of 59 patients, we applied a novel supervised learning algorithm to build a gene expressionbased clinical outcome predictor, which was then tested using an independent validation group comprising the 57 remaining patients. An optimal 133-gene expression signature accurately predicted overall survival for patients in the independent validation group $(\mathrm{P}=0.006)$, even for the subset of AML cases with normal karyotype alone $(\mathrm{P}=0.046)$. In multivariate analysis, the gene-expression predictor was a strong [odds ratio $=8.8$ (2.6 to 29$) ; \mathrm{P}<0.001]$, independent prognostic factor. Our findings support the utility of gene expression profiling for improved molecular classification and disease management in adult AML.

\section{L170}

Comparative genomic hybridisation on to array slides reveals specific DNA copy number changes in acute lymphoblastic leukaemia

\author{
J. Strefford ${ }^{1}$, M. Griffiths ${ }^{2}$, F. Ross ${ }^{3}$ and \\ C. Harrison ${ }^{1}$ \\ ${ }^{1}$ Leukaemia Research Fund Cytogenetics Group, \\ Cancer Sciences Division, University of \\ Southampton, UK; ${ }^{2}$ Regional Genetics \\ Laboratory, Birmingham Women's Hospital, UK; \\ ${ }^{3}$ Wessex Regional Genetics Laboratory, Salisbury \\ General Hospital, UK
}

An important factor in the diagnosis of acute lymphoblastic leukaemia (ALL) is that karyotype is an independent prognostic indicator, with an impact on the choice of treatment. Although patient outcome can be related to changes in chromosome number and structure, there remain instances where no established chromosome abnormality is available to aid prognostication. The aim of this study is to investigate the DNA from patients without informative cytogenetic data using microarray-based comparative genomic hybridization (aCGH), to allow the identification of novel chromosome regions involved in 
disease development, progression and patient outcome. We have used $1 \mathrm{Mb}$ aCGH analysis to accurately define DNA copy number changes throughout the genome in DNA from 50 ALL patients and compared it with previous cytogenetic and molecular cytogenetic information. Although this project is ongoing, aCGH analysis has already allowed the accurate mapping of several previously described cytogenetic abnormalities and highlighted several novel regions. For example, the size of $9 p$ deletions varied from a single DNA clone (including the p16 gene locus) to large deletions involving loss of 30-40 DNA clones. Small novel changes involving two or three DNA clones were also identified on other chromosomes and may harbour important tumour suppressor and oncogenes. In general, aCGH is valuable in the characterisation of complex karyotypes and the data compares well with conventional G-banded, FISH and MFISH analysis. In the future, this type of genetic information may be important in the development of effective treatment strategies and defining therapeutic targets.

\section{L171}

The characteristic trisomies of childhood high hyperdiploid ALL are associated with an increase in expression of associated sequences

L. Kearney ${ }^{1}$, S. Horsley ${ }^{1}$, A. Martinez Ramirez $^{1}$, C. Harrison ${ }^{2}$, H. Kempski ${ }^{3}$, A. Moorman' ${ }^{2}$ F. Ross ${ }^{4}$, M. Griffiths ${ }^{5}$, M. Greaves ${ }^{1}$ and A. Gruszka-Westwood ${ }^{1}$

${ }^{1}$ Section of Haematological Oncology, Institute of Cancer Research, London, UK; ${ }^{2}$ LRF Cytogenetics Group, Cancer Sciences Division, Southampton General Hospital, Southampton UK; ${ }^{3}$ LRF Centre for Childhood Leukaemia, Institute of Child Health, London, UK; ${ }^{4}$ Wessex Regional Genetics

Laboratory, Salisbury District Hospital, Salisbury UK; ${ }^{5}$ Regional Genetics Laboratory, Birmingham Women's Hospital, Birmingham, UK

High hyperdiploidy, defined as 51-67 chromosomes per malignant cell, occurs in $30 \%$ of childhood acute lymphoblastic leukaemia (ALL) cases. However, neither the causative mechanism, nor the relevant molecular consequences of extra chromosomes on leukaemogenesis are known. To address the question of whether the characteristic trisomies in high hyperdiploid ALL are associated with increased gene expression, we used comparative expressed sequence hybridisation (CESH), a technique analogous to CGH that identifies chromosomal regions with differential gene expression. Relative expression of ALL blast cells versus peripheral blood mononuclear cells was analysed in 18 high hyperdiploid ALL patients. CESH profiles showed an increase in expression for the trisomic chromosomes, with distinct peaks observed at: Xp22.1-22.2, 4q28, 6q14-15, 6q24, 10 p13, 14q23-24, 17q21, 18q12, 21q21 in $28-89 \%$ of cases. Importantly, increased expression without underlying trisomy occurred at $7 \mathrm{q} 11.2$ in $90 \%$ of cases. This was confirmed by quantitative PCR. When expression was reanalysed versus a range of purified normal cell fractions as reference, a reduction in the number of relatively over-expressed regions was observed with CD34+ 19+ cells, the putative normal counterpart for the ALL blast cell. In particular, the expression peak at 7q11.2 disappeared, indicating the involvement of genes within this region in the early ontology of normal B-cell development. Overall, this study has shown that the characteristic trisomies in hyperdiploid ALL lead to increase in expression of associated sequences, and has highlighted critical regions for further investigation. The importance of choosing the biologically relevant reference cell type for meaningful data interpretation has also been demonstrated.

\section{L172}

\section{Genetic mapping and expression} analyses of MYC-containing double minutes in myeloid malignancies: detection of a commonly amplified 4.3 Mbregion with overexpression of the C8FW gene but not of the MYC gene

C. Storlazzi ${ }^{1}$, T. Fioretos ${ }^{2}$, K. Paulsson ${ }^{2}$, B. Strombeck ${ }^{2}$, C. Lassen ${ }^{2}$, T. Ahlgren ${ }^{3}$, G. Juliusson ${ }^{4}$, F. Mitelman ${ }^{2}$, M. Rocchi ${ }^{1}$ and B. Johansson ${ }^{2}$

${ }^{1}$ DAPEG, Section of Genetics, University of Bari, Italy; ${ }^{2}$ Department of Clinical Genetics, Lund 
University Hospital, Sweden; ${ }^{3}$ Department of Hematology, Malmö University Hospital, Sweden; ${ }^{4}$ Department of Hematology, Linköping University Hospital, Sweden;

Double minutes (dmin), the cytogenetic hallmark of genomic amplification, are found in approximately $1 \%$ of karyotypically abnormal acute myeloid leukemias (AML) and myelodysplastic syndromes (MDS). The MYC gene at 8q24 has been reported to be amplified in the majority of the cases, and it has been generally assumed that MYC is the target gene. However, only a few studies have focused on the extent of the amplicon or on the expression patterns of the amplified genes. We have studied 6 AML and MDS cases with MYC-containing dmin. Detailed fluorescence in situ hybridization analyses identified a common 4.3 $\mathrm{Mb}$ amplicon, with clustered proximal and distal breakpoints, harboring 8 known genes (C8FW, NSE2, POU5FLC20, MYC, PVT1, AK093424, MGC27434, and MLZE). The corresponding region was deleted in one of the chromosome 8 homologues in 5 of the 6 cases, suggesting that the dmin originated through extra replication (or loop-formation)-excision-amplification. Northern blot analysis revealed that MYC was not overexpressed. Instead, only the $\mathrm{C} 8 \mathrm{FW}$ gene, encoding a phosphoprotein regulated by mitogenic pathways, displayed increased expression. These results exclude MYC as the target gene and strongly indicate that deregulation of the C8FW gene may be the functionally important consequence of 8q24 amplicons in AML and MDS.

\section{L173}

\section{Detection of new specific chromosome aberrations in acute leukaemia using molecular cytogenetic techniques}

\section{Van Zutven', E. Van Drunen ${ }^{1}$, S. Velthuizen ${ }^{1}$ and B. Beverloo ${ }^{2}$}

${ }^{1}$ Department of Genetics, Erasmus MC, Rotterdam, The Netherlands; ${ }^{2}$ Department of Clinical Genetics, Erasmus MC, Rotterdam, The Netherlands

Specific chromosome aberrations are observed in $50 \%$ of acute leukaemia patients. In the other $50 \%$ no or non-specific aberrations are found. It might very well be that in this group cryptic specific chromosome abnormalities are present that are not detectable using conventional karyotyping. We used spectral karyotyping (SKY) and comparative genomic hybridisation $(\mathrm{CGH})$ to identify new specific chromosome rearrangements.

In 30 AML patients, frequent structural involvement of chromosomes 3, 5, 11 and 12 was observed with karyotyping only. Additionally, SKY showed frequent rearrangements of chromosomes 5 and 17. In 14 ALL patients, chromosomes 9 and 22 were frequently involved based on karyotyping only. SKY showed frequent involvement of chromosomes 12 and 21 as well. In 16 MDS cases we found many abnormalities of chromosomes 1, 2, 3, 5, 7 and 15. Using SKY, new structural aberrations were found involving chromosomes 17 and 22. In 3 cases we observed rearrangements of apparently normal chromosomes: a $\operatorname{der}(1) \mathrm{t}(1 ; 22)$ and $\operatorname{der}(21) \mathrm{t}(5 ; 21)$ in $\mathrm{ALL}$, a $\operatorname{der}(10) \mathrm{t}(10 ; 20)$ in MDS and a der(2)t $(2 ; 3)$ in AML. In patients having rearrangements of the same chromosomes, as observed using SKY, CGH was started to study the chromosomal regions involved. Regions of $5 \mathrm{q}$, $7 q$ and $17 q$ are frequently lost, suggesting that genes present in a minimally lost region might be important for leukemogenesis. Therefore, studying these regions and the newly observed translocations in more detail might lead to the identification of new specific chromosome aberrations and genes that might play a role in leukemogenesis.

\section{L174}

\section{Development and application of two dual colour split signal FISH assays for detection of the $t(5 ; 14)$ involving HOX11 L2 or CSX in T-cell acute lymphoblastic leukaemia}

\author{
L. Van Zutven'1, S. Velthuizen', \\ I. Wolvers-Tettero' ${ }^{2}$ J. Van Dongen²,

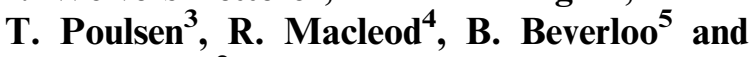 \\ A. Langerak ${ }^{2}$ \\ ${ }^{1}$ Department of Genetics, Erasmus MC, \\ Rotterdam, The Netherlands; ${ }^{2}$ Department of \\ Immunology, Erasmus MC, Rotterdam, The \\ Netherlands; ${ }^{3}$ Department of Probe Application, \\ DakoCytomation, Glostrup, Denmark;
}


${ }^{4}$ Department of Human and Animal Cell Culture, DSMZ, Braunsweig, Germany; ${ }^{5}$ Department of Clinical Genetics, Erasmus MC, Rotterdam, The Netherlands

The $\mathrm{t}(5 ; 14)(\mathrm{q} 35 ; \mathrm{q} 32)$ is a novel cryptic translocation recently described in paediatric T-cell acute lymphoblastic leukaemia (T-ALL). This translocation involves either HOX11L2 or CSX on 5q35, whereas the $14 \mathrm{q} 32$ breakpoints are very heterogeneous. Because the $t(5 ; 14)(\mathrm{q} 35 ; \mathrm{q} 32)$ is cryptic and thus hard to detect using conventional karyotyping, it is easily missed in routine diagnostics. Here we describe the development, validation and application of split signal FISH assays for both HOX11L2 and CSX, for easy metaphase and interphase detection of $t(5 ; 14)$ possibly present in T-ALL patients. We also used a split signal probe combination for the possible involvement of IGH on $14 \mathrm{q} 32$. We identified 5 new cases with a $\mathrm{t}(5 ; 14)$ involving HOX11L2 out of 32 new T-ALL cases; in each case the $14 \mathrm{q}$ breakpoint was found to be centromeric of the IGH region. All 5 positive cases showed HOX11L2 expression. Additionally, HOX11L2 expression was observed in 1 case without the $\mathrm{t}(5 ; 14)(\mathrm{q} 35 ; \mathrm{q} 32)$, showing that HOX11L2 expression may occur independently of $t(5 ; 14)$ (q35;q32). We did not identify patients with a $\mathrm{t}(5 ; 14)(\mathrm{q} 35 ; \mathrm{q} 32)$ involving CSX, indicating that the incidence of this alternative $5 \mathrm{q}$ translocation is probably low. We also found that cases with the $\mathrm{t}(5 ; 14)(\mathrm{q} 35 ; \mathrm{q} 32)$ involving HOX11L2 did not show TAL1 abnormalities, whereas 5 HOX11L2 negative cases did. These results suggest that TAL1 expression and HOX11L2 expression, or TAL1 aberrations and the $\mathrm{t}(5 ; 14)(\mathrm{q} 35 ; \mathrm{q} 32)$ trans location involving HOX11L2 are mutually exclusive.

\section{PO:08:84}

\section{Genomic deletions on other} chromosomes involved in variant t(9;22) chronic myeloid leukemia cases

L. Anelli ${ }^{1}$,A.Zagaria ${ }^{1}$,F. Albano ${ }^{2}$,C.Storlazzi ${ }^{3}$, A. Pannunzio ${ }^{2}$, C. Buquicchio ${ }^{2}$, M. Roberti ${ }^{1}$, V. Liso ${ }^{2}$, G. Specchia ${ }^{1}$ and M. Rocchi ${ }^{3}$

${ }^{1}$ Hematology, University of Foggia, Foggia, Italy; ${ }^{2}$ Department of Hematology, University of Bari,
Bari, Italy; ${ }^{3}$ Sezione di Genetica, DAPEG, University of Bari, Bari, Italy

The Philadelphia chromosome $(\mathrm{Ph})$, due to $\mathrm{t}(9 ; 22)(\mathrm{q} 34 ; \mathrm{q} 11)$, is observed in more than $95 \%$ of chronic myeloid leukemia cases. Large deletions adjacent to the translocation junction on the derivative 9 chromosome have recently been identified in patients with CML. We report the presence of similar deletions on the third derivative other than chromosomes 9 and 22 in $4 \mathrm{CML}$ cases with variant translocations using fluorescence in situ hybridization (FISH).

We studied 11 cases affected by CML with variant translocations at diagnosis. Of the 11 cases, 7 (64\%) bore large deletions on der(9). Among them, we found 4 cases $(57 \%)$ with microdeletions also on the third derivative chromosome: $\mathrm{t}(6 ; 9 ; 22)$ (p12;q34;q11), $\mathrm{t}(9 ; 13 ; 22)(\mathrm{q} 34 ; \mathrm{q} 14 ; \mathrm{q} 11), \quad \mathrm{t}(4 ; 9 ; 22)$ (p15;q34;q11), t(9;11;22)(q34;q13;q11) in cases \#1, $\# 2$, \#3, and \#4, respectively. These cases were characterized in detail by FISH analysis with appropriate P1 Artificial Chromosome (PAC) or Bacterial Artificial Chromosome (BAC) clones. This study showed an association between deletions on $\operatorname{der}(9)$ and the loss of genomic sequences of other chromosomes involved in variant $t(9 ; 22)$. The deletions were detected in each analyzed metaphase cell, suggesting that, very likely, the rearrangement and the deletion occurred at the same time. We precisely defined the extension of genomic material losses. The size of the deletions at the third chromosome appears to be relatively large, ranging from 2.7 to $20.4 \mathrm{Mb}$. Moreover, the deleted sequences on the third derivative chromosomes included tumor suppressor genes and/or other genes involved in signal transduction or in modulation of cell proliferation. These findings may yield further information about the pathogenesis of CML.

\section{PO:08:85}

Systematic screening for MLL status at diagnosis in 209 unselected patients with acute myeloid leukemia
B. Arnaud ${ }^{1}$, N. Douet-guilbert ${ }^{1}$, F. Morel ${ }^{1}$, M. Lebris ${ }^{1}$, P. Morice ${ }^{2}$, P. Bourquard ${ }^{2}$, S. Banzakour ${ }^{2}$, C. Berthou ${ }^{1}$, J. Abgrall ${ }^{1}$ and M. De Braekeleer ${ }^{1}$ 
${ }^{1}$ Faculté de Médecine \& CHU Morvan, Brest, France; ${ }^{2} \mathrm{CH}$ Yves Le Foll, St Brieuc, France

A large number of abnormalities involving the MLL gene, located in the 11q23 chromosomal band, has been associated with hematological malignancies, including acute lymphoblastic and myeloblastic leukemias and myelodysplastic syndromes. In this study, we investigated the occurrence of MLL abnormalities in 209 unselected consecutive patients with acute myeloblastic leukemia (AML) using fluorescent in situ hybridization (FISH) analyses with a commercial MLL dual colour probe (LSÏ MLL, Vysis). Twenty-six patients $(12.4 \%)$ showed MLL abnormalities. Nine showed a split signal ascertaining the involvement of the MLL gene in translocations (7 cases) and insertions ( 2 cases). FISH analysis was necessary to identify the partner chromosome or the exact mechanism of the disruption for 4 of the patients. Seventeen patients showed overrepresentation of the MLL gene without evidence of rearrangement. Trisomy and tetrasomy 11 were observed in 6 and 1 cases respectively. Other various abnormalities, such as double minutes and i(11) (q10), were the source of the MLL amplification. MLL rearrangements have been much studied for a long time and shown to be associated with a worse prognosis. However, MLL amplification has only been considered as a new entity in AML quite recently, playing a putative role in leukemogenesis. Given the cryptic nature of some rearrangements and the difficulty of identifying chromosome 11 segments in some abnormalities, we think that FISH analysis with the MLL probe should be performed in all AML cases at diagnosis.

\section{PO:08:86}

\section{MLL-partner fusion and transformation activity in donor cells after allogeneic transplant in two patients}

\author{
S. Brodie ${ }^{1}$ and N. Rao ${ }^{1}$ \\ ${ }^{1}$ UCLA School of Medicine, Los Angeles, CA \\ 90095, USA
}

Treatment for hematologic disease often includes allogeneic bone marrow transplantation after successful induction chemotherapy and consolidation. Post-transplant leukemic relapse occurs when residual, therapy resistant host cells proliferate leading to recurrence of the original or further progressed disease. However, successful eradication of recipient tumor cells and subsequent transformation of donor cells is uncommon. Here we report two cases of AML in donor cells after related sex mis-matched allogeneic bone marrow transplantation. The first case was a 55-year old female with AML-M2 carrying a 8;21 (ETO;AML1) translocation. She responded well to treatment and transplant. However, after 2 years she relapsed and developed cytopenia with a karyotype showing a $(11 ; 19)(\mathrm{q} 23 ; \mathrm{p} 13.3)$ translocation and trisomy 21 in all the male donor cells. No female cells were detected by XY FISH studies. The second case was a 37-year-old female diagnosed with paroxysomal nocturnal hemoglobinuria and had a normal karyotype. Her course was complicated by neutropenic fevers and, graft versus host disease. She relapsed and developed leukopenia. This time her karyotype showed trisomy 11 and $\mathrm{t}(11 ; 21)(\mathrm{q} 23.3 ; \mathrm{q} 22)$ in all the male donor cells. FISH analyses on both cases suggested aberrations involving the MLL gene. Expression of chimeric MLL fusion products or additional copies of MLL gene have been associated with transformation. These two cases illustrate the unusual occurrence of transformation of donor cells by MLL-partner fusion proteins.

\section{PO:08:87}

\section{Identification of chromosomal aberrations in chronic lymphatic leukemia (CLL) by interphase FISH and multiplex ligation-dependent probe amplification (MLPA).}

\author{
A. Buijs ${ }^{1}$ and P. Krijtenburg ${ }^{1}$ \\ ${ }^{1}$ Department of Medical Genetics, University \\ Medical Centre Utrecht, The Netherlands
}

Chronic lymphatic leukemia (CLL) is the most common leukemia in adults. It has a highly variable clinical course. Attempts to correlate this 
apparent clinical heterogeneity to genomic heterogeneity have been hampered by poor cytogenetics of CLL samples. To identify new markers we analyzed 41 CLL cases using multiplex ligation-dependent probe amplification (MLPA) for 41 tumor-associated targets. To validate the MLPA analysis, MLPA data of CLL samples were compared with results of interphase FISH analysis using ATM (11q22-23), centromere 12 and Rb-1 (13q14) specific probes.

We found that $3 / 29$ cases had a deletion of the ATM locus by FISH that could be confirmed by MLPA. In 6/8 cases with a trisomy chr.12 seen by FISH, the result was confirmed by MLPA analysis using target sequences specific for CCND2 and BCL7A, located on chromosome 12. In two cases, the percentages of interphase nuclei with +12 were 20 and $40 \%$, respectively, which may explain the false-negative result for MLPA. In one case, MLPA confirmed trisomy chr.12, but also suggested a trisomy chr.19. This was confirmed by M-FISH. Of 10 cases with a Rb-1 deletion by FISH analysis out of 41 cases, 8 could be confirmed by MLPA. In two cases the deletion of Rb-1 was found only in $15 \%$ and $30 \%$ of the interphase nuclei that may explain the false negative results by MLPA analysis.

We conclude that MLPA can be used to identify new chromosomal aberrations that serve as markers for the identification of independent predictors of disease and survival in CLL.

\section{PO:08:88}

\section{Does elevated survivin level influence the progress of B-cell lymphocytic leukemia?}

A. Filip ${ }^{1}$, D. Koczkodaj ${ }^{1}$, I. Nesina ${ }^{1}$, B. Cisel ${ }^{1}$, J. Korzeniowska ${ }^{1}$, E. Wasik-Szczepanek ${ }^{2}$, A. Dmoszynska ${ }^{2}$ and J. Wojcierowski ${ }^{1}$

${ }^{1}$ Department of Human Genetics with Molecular and Cytogenetic Diagnostics, Lublin, Poland; ${ }^{2}$ Department of Haematooncology and Bone Marrow Transplantation, Lublin, Poland

Survivin is a member of IAP (inhibitor of apoptosis) gene family. Absent in almost all tissues except foetal, survivin is often overexpressed in human tumors. Chronic lymphocytic leukemia is unique among other malignancies, for survivin mRNA and protein is undetectable in leukemic B lymphocytes.

We present a case of B-CLL patient that expressed survivin when analyzed by RNase Protection Assay.

Utilizing FISH technique we also found chromosome 12 trisomy, deletion in p53 locus and 13q deletion.

Diagnosed with stadium III according to Rai classification, this patient showed slow disease progression and poor chemotherapy response.

\section{PO:08:89}

\section{Trisomy 12 in B-cell chronic} lymphocytic leukemia detected by fluorescence in situ hybridization

\section{Koczkodaj ${ }^{1}$, E. Wasik-Szczepanek ${ }^{2}$,} A. Filip ${ }^{1}$, A. Dmoszynska ${ }^{2}$ and

J. Wojcierowski ${ }^{1}$

${ }^{1}$ Department of Human Genetics with Molecular and Cytogenetic Diagnostics, Lublin, Poland; ${ }^{2}$ Department of Haemato-oncology and Bone Marrow Transplantation, Lublin, Poland

Among 75 untreated patients with typical $(\mathrm{CD} 19+, \mathrm{CD} 5 / \mathrm{CD} 19+, \mathrm{CD} 23 / \mathrm{CD} 19+) \mathrm{B}$-cell chronic lymphocytic leukemia (B-CLL) cytogenetic aberrations of peripheral blood cells were evaluated, using fluorescence in situ hybridization techniques. Two cytogenetic aberrations were found: trisomy 12 and TP53 deletion. The clonality was determined when $>9 \%$ of the cells had trisomy 12 or TP53 gene deletion. Trisomy 12 was detected in 7 patients, while trisomy 12 and TP53 deletion were present simultaneously in 6 patients. If the first group is linked to the second one then 13 patients among $75(17 \%)$ have trisomy 12 . In a group of patients with trisomy 12 and TP53 deletion, the percentage of cells with trisomy 12 was almost two times more compared to patients with trisomy 12 as a single aberration. It is possible that TP53 deletion facilitates proliferation of clones with others genomic aberrations. In two patients with trisomy 12 , control cytogenetic study was performed. An increase in 
the percentage of cells with trisomy 12 for $8 \%$ and $30 \%$, respectively was detected. However, proliferation of cells with TP53 deletion was also observed. Clinical course of B-CLL in a group of patient with trisomy 12, trisomy 12 and TP53 deletion simultaneously is more aggressive compared to the course of the disease in patients with no cytogenetic aberrations. Frequency of IGHV gene mutation occurrence was not analyzed in both groups of patients. But trisomy 12 together with unmutated IGHV gene is found by some authors. The absence IGHV gene mutation is independent unfavourable prognostic factor.

\section{PO:08:90}

\section{Karyotyping and FISH in} haematological malignancies in India

P. Madon', F. Parikh', A. Athalye ${ }^{1}$, V. Bandkar ${ }^{1}$, S. Dhumal ${ }^{1}$, A. Sopariwala ${ }^{1}$, M. Kawle ${ }^{1}$, S. Advani ${ }^{1}$, S. Parekh ${ }^{2}$ and M. Agarwal ${ }^{2}$

${ }^{1}$ Jaslok Hospital and Research Centre, Mumbai, India; ${ }^{2}$ Bombay Hospital and Medical Research Centre, Mumbai, India

Cytogenetic analysis was performed on over 1000 samples of bone marrow or peripheral blood at the Genetics laboratory of a private hospital in Mumbai. FISH was carried out using Vysis probes, the signals were visualized on a Zeiss Axioskop microscope and the Metasystems software was used for image analysis. The use of both karyotyping and FISH gave additional information especially when complex translocations were present in CML, leading to masked Philadelphia chromosomes. FISH on metaphases helped to detect a case where the BCR/ABL fusion signal was present on chromosome 9 instead of 22 . Karyotyping a patient in the blast phase showed dup(1)(q22q24) with hyperdiploidy and two $\mathrm{Ph}$ chromosomes. Clonal evolution was detected with trisomy 4, del(5)(q22-q33), del(7)(q22-q36), der(17) or $i(17 q)$ in different cases of CML. The BCR/ABL ES probe (Vysis) was used to detect deletions in the ASS region. In cases of sex-mismatched bone marrow transplantation, FISH using probes for the sex chromosomes determined success or relapse. Patients with CML and AMLM3 were managed by repeatedly monitoring the success of treatment by FISH.

In ALL, some of the cytogenetic abnormalities detected were $\mathrm{t}(1 ; 19)(\mathrm{q} 23 ; \mathrm{p} 13)$, a jumping translocation $\mathrm{t}(5 ; 12), \mathrm{i}(6 \mathrm{p})$ and trisomy 8 . A complex translocation $\mathrm{t}(2 ; 21 ; 14)(\mathrm{p} 12 ; \mathrm{p} 11 ; \mathrm{q} 32)$ was seen in a case of CLL. A patient with ATLL had $\mathrm{t}(1 ; 3)(\mathrm{p} 32 ; \mathrm{p} 21)$ with trisomy 4 , trisomy 12 and inversion 6. Cytogenetic studies thus help in the diagnosis, prognosis and management of patients with leukemia.

\section{PO:08:91}

\section{Chromosome 1 abnormalities in multiple myeloma: a multicolor FISH study.}

Y. Marzin ${ }^{1}$, D. Jamet ${ }^{1}$, F. Morel ${ }^{1}$, M. Lebris ${ }^{1}$, P. Morice $^{2}$, P. Bourquard ${ }^{2}$, S. Banzakour ${ }^{2}$, C. Berthou ${ }^{1}$, J. Abgrall ${ }^{1}$ and M. De Braekeleer ${ }^{1}$

${ }^{1}$ Faculté de Médecine \& CHU Morvan, Brest, France; ${ }^{2} \mathrm{CH}$ Yves Le Foll, St Brieuc, France

Multiple myeloma (MM) is a B cell neoplasia characterized by the proliferation of a malignant plasma cell population in the bone marrow. Patients with MM commonly present bone pain, anemia, renal failure and paraproteinemia. Given the karyotypic complexity observed in most patients with advanced disease, conventional cytogenetic techniques usually fail to properly identify all the chromosome abnormalities. This is why biomolecular techniques, such as multicolor fluorescent in situ hybridization (M-FISH), are used to improve the interpretation of the karyotypes. Amongst the 56 patients studied since 2000, 37 presented chromosome 1 abnormalities. Most of these abnormalities were unbalanced translocations resulting in amplifications of the long arm (22 patients) or deletions of the short arm (12 patients). The minimum regions of overlap were 1q31q4 and $1 \mathrm{p} 11 \mathrm{p} 21$ for the amplifications (20 patients) and deletions (9 patients) respectively. The 31 breakpoints (18 patients) on the chromosome partners of the unbalanced translocations were randomly distributed on the 
whole karyotype, but for 6 cases located on chromosome 16, including 5 in band 16q11. Almost half of the breakpoints occurred in the pericentromeric regions. Balanced translocations involving chromosome 1 were also distributed in the whole karyotype and half of the breakpoints occurred in pericentromeric regions. Nevertheless, such translocations were rare (6 of the 37 patients), interesting the long or short arm without predominance. As chromosome 1 abnormalities are commonly considered of bad prognosis in $\mathrm{MM}$, it is important to identify these rearrangements, which can be achieved by M-FISH.

\section{PO:08:92}

A translocation $\mathrm{t}(5 ; 12)(\mathrm{q} 33 ; \mathrm{p} 13.1)$ in a chronic myeloproliferative disease without eosinophilia

\author{
N. Nadal ${ }^{1}$, P. Flandrin ${ }^{1}$, J. Cornillon ${ }^{2}$, \\ E. Deladesse ${ }^{3}$, L. Mauvieux ${ }^{4}$, D. Olaru ${ }^{1}$, \\ S. Morel ${ }^{1}$ and L. Campos ${ }^{1}$
}

${ }^{1}$ Laboratoire d'Hématologie, CHU Hopital Nord, 42055 Saint-Etienne, France; ${ }^{2}$ Service d'Hématologie, CHU Hopital Nord, 42055 Saint-Etienne, France; ${ }^{3}$ Laboratoire Central d'Hématologie, CHU Necker-Enfants-Malades, 75015 Paris, France; ${ }^{4}$ Laboratoire d'Hématologie, CHU de Hautepierre, 67000 Strasbourg, France

A $t(5 ; 12)(q 33 ; p 13)$ has been described in a small number of cases of BCR-ABL negative (Ph-) Chronic MyeloProliferative Disease (CMPD) with eosinophilia. The translocation $t(5 ; 12)$ fuses the Platelet-Derived Growth Factor Receptor (PDGFR) gene at 5q31-33 to the ETS-Variant gene 6 (TEL/ETV6) at 12p13.

We report a case of Ph- CMPD without eosinophilia associated with an isolated $\mathrm{t}(5 ; 12)(\mathrm{q} 33 ; \mathrm{p} 13)$ observed in 25 metaphase cells. Molecular analysis detected the TEL-PDGFR fusion gene and showed the absence of a cryptic BCR-ABL rearrangement. The diagnosis was atypical $\mathrm{Ph}$ CMPD. A good haematological and cytogenetic response was obtained after treatment with Imatinib Mesylate. The patient was in good health after one year of treatment.

$\mathrm{Ph}-\mathrm{CMPD}$ is a heterogeneous group of disease. A minority of cases are associated with a recur- rent reciprocal translocation. The review of the literature suggests the definition of a new subgroup of Ph- CMPD which includes t(5;12)(q33;p13), eosinophilia, monocytosis and splenomegaly. Our case is one of the few not associated with eosinophilia, which suggest that eosinophilia is a prominent but not invariable feature.

The TEL-PDGFR fusion gene results in production of a constitutively active tyrosine kinase fusion protein that deregulates hemopoiesis. With the advent of targeted treatment, patients with $\mathrm{t}(5 ; 12)$ are candidates for treatment with Imatinib Mesylate, a tyrosine kinase inhibitor. The treatment induces durable responses in patients in the literature and in our case.

\section{PO:08:93}

\section{Variations of CCND1/IGH signal pattern in Mantle Cell Lymphoma (MCL) by double-colour fluorescence in situ hybridization (FISH)}

R. Woroniecka ${ }^{1}$, B. Pienkowska-Grela ${ }^{1}$, A. Pastwinska $^{1}$, J. Rygier ${ }^{1}$ and A. Witkowska ${ }^{1}$

${ }^{1}$ Centre of Oncology, M. Sklodowska-Curie Memorial Institute, Cytogenetic Department, Warsaw, Poland

Mantle cell lymphoma is characterised by $\mathrm{t}(11 ; 14)(\mathrm{q} 13 ; \mathrm{q} 32)$. This translocation juxtaposes IGH sequences with the CCND1 (BCL-1). Classic cytogenetic analysis of MCL is frequently impossible, due to low mitotic index and poor morphology of metaphases. FISH detects the aberrations on interphases as well as metaphases. We investigated this method in the new diagnosed MCL patients.

FISH analysis was carried out on lymphoid cells obtained from lymph node, bone marrow or peripheral blood. All known breakpoints of $t(11 ; 14)$ were spanned by the use of two dual colour probes: LSI IGH/CCND1 (Vysis) and BCL1/IGH (Qbiogene).

FISH study was carried out on 14 MCL cases. The typical 1R1G2Y of signals was observed in 11 cases. Simultaneously, in 3 of them a subpopulation of cells with different signal pattern was observed. There were detected 2R2G3Y, 
2R2G4Y, that indicated tetraploidy or $1 \mathrm{R} 1 \mathrm{G} 3 \mathrm{Y}$, $1 \mathrm{R} 1 \mathrm{G} 4 \mathrm{Y}$, that suggested amplification of fusion gene. In one of the remaining 3 cases the signal pattern was 1 R $1 \mathrm{G} 1 \mathrm{Y}$. The analysis of metaphases showed, that the missing yellow fusion signal on the derivative chromosome 11 was probably deleted. In the next case the signal pattern was $2 \mathrm{R} 2 \mathrm{G}$ and $2 \mathrm{R} 3 \mathrm{G}$. The detailed analysis of metaphases indicated a variant of translocation of CCND1 gene onto chromosome 2. In the last case hybridization demonstrated the lack of fusion signals and $2 \mathrm{R} 2 \mathrm{G}$ pattern.

FISH is enough not only for detection of the CCND1/IGH fusion, but also for detection of deletions or amplifications associated with the CCND1/IGH fusion.

\section{PO:08:94}

Insertions generating the 5/RUNX1/3/CBFA2T1 gene in acute myeloid leukemia cases show variable breakpoints

\section{A. Zagaria ${ }^{1}$, L. Anelli ${ }^{1}$, F. Albano ${ }^{2}$,} M. Mancini ${ }^{3}$, G. Saglio ${ }^{4}$, A. Liso ${ }^{1}$, L. Sebastio, R. La Starza, G. Specchia ${ }^{1}$ and M. Rocchi ${ }^{5}$

${ }^{1}$ Hematology, University of Foggia, Foggia, Italy; 2Department of Hematology, University of Bari, Bari, Italy; ${ }^{3}$ Dipartimento di Biotecnologie Cellulari ed Ematologia, University La Sapienza, Rome, Italy; ${ }^{4}$ Division of Hematology and Internal Medicine, Department of Clinical and Biological Sciences of the University of Turin, Turin, Italy; ${ }^{5}$ Sezione di Genetica, DAPEG, University of Bari, Bari, Italy

Translocation $\mathrm{t}(8 ; 21)(\mathrm{q} 22 ; \mathrm{q} 22)$ is detected in about $15 \%$ of acute myeloid leukemia cases. The rearrangement involved RUNX1 and CBFA2T1 genes generating a 5RUNX1/3CBFA2T1 fusion gene. We described 6 among 82 (7.3\%) AML cases showing insertion events responsible for the RUNX1/CBFA2T1 rearrangement. All cases were studied by RT-PCR and conventional cytogenetic analysis. A detailed molecular cytogenetic characterization by FISH experiments revealed 1 case with ins(8;21) and 5 with ins(21;8). In detail, the CBFA2T1 locus was identified using a mixture of RP11-118O8 and RP11-777J24 clones, completely encompassing the gene; RUNX1 gene was detected by RP5-1107L6 probe. In the case with ins $(8 ; 21)$, the use of chromosome 21 specific clones revealed that a region of $4 \mathrm{Mb}$ was inserted on the der(8) chromosome. In the remaining cases a functional fusion gene was detected on the der(21) instead of the der(8) chromosome as a consequence of ins $(21 ; 8)$. The insertion size was established in all cases and turned out to be very heterogeneous, ranging from a minimum of $2.4 \mathrm{Mb}$ to a maximum of $44 \mathrm{Mb}$. Sequences encompassing the breakpoints on chromosome 8 were compared each other and with the region including RUNX1 gene on chromosome 21, using the Genalyzer software. No significant similarity was observed.

Insertions seem not to be associated with a subset of patients with common features in terms of prognosis and are not linked to the presence of additional cytogenetic rearrangements. Our findings suggested that the 5RUNX1/3CBFA2T1 fusion gene plays a crucial role in leukemogenesis independently from breakpoint location and insertion size.

\section{Post-genomic Era Symposium}

\section{L175 \\ Chromosomes in disarray: DNA microarrays for the study of chromosome rearrangements}

\section{N. Carter ${ }^{1}$}

${ }^{1}$ The Wellcome Trust Sanger Institute, Hinxton, Cambridge, UK

With the publication of the complete human genome sequence, a complete set of mapped and sequenced DNA clones are now available which can be utilised as a valuable resource for molecular cytogenetics. The use of this resource in the form of DNA microarrays to represent and replace metaphase chromosomes provides us with new possibilities for the analysis of chromosomes and their aberrations. We have been using BAC and PAC clone DNA microarrays not only to study gains and losses in tumours and to identify microdeletion and microduplications in patients with learning disability using comparative 
genomic hybridisation (CGH) but also to map chromosome translocation breakpoints.

For breakpoint mapping, the derivative chromosomes are first flow sorted, amplified using DOP-PCR, differentially labelled and hybridised to the array. The pattern of hybridisation identifies the chromosomes involved in the rearrangement and the position of the breakpoint. This new approach, which we have termed Array Painting, dramatically reduces the time taken to map breakpoints by FISH. We have used these methods to identify additional complexity or genome imbalance in $60 \%$ of patients with apparently balanced rearrangement. These results, if generally confirmed in the study of further patients, will have a significant impact on current diagnostic investigations of this type and provide an argument for the more widespread adoption of microarray analysis or other high resolution genome wide screens for chromosome imbalance and rearrangement.

\section{L176}

Array painting, array CGH and FISH reveal complex chromosomal abnormalities in a proportion of patients with apparently balanced de novo translocations and abnormal phenotypes

J. Crolla ${ }^{1}$, N. Carter ${ }^{2}$, S. Gribble ${ }^{2}$, E. Prigmore ${ }^{2}$, D. Burford ${ }^{2}$, S. Clegg ${ }^{2}$, N. Dennis ${ }^{3}$, P. Jacobs ${ }^{1}$, S. Thomas ${ }^{1}$ and R. Sandstrom ${ }^{4}$

${ }^{1}$ Wessex Regional Genetics Laboratory, Salisbury District Hospital, Salisbury, SP2 8BJ. U.K.; ${ }^{2}$ The Wellcome Trust Sanger Institute, Wellcome Trust Genome Campus, Hinxton, Cambridge, CB10

1SA. U.K.; ${ }^{3}$ Wessex Clinical Genetics Service, Princess Anne Hospital, Coxford Road,

Southampton, SO16 5YA. U.K.; ${ }^{4}$ Lower Columbia Pathologists, 1217 14th Ave, Longview, WA, U.S.A.

We have used array-CGH and array painting to analyse constitutional de novo apparently balanced translocations in ten patients presenting with abnormal phenotypes. With this approach we identified additional complexity or genome imbalance in six of the ten patients analysed with- out knowledge of their phenotypes. These results sub-divided the patients into three groups. (1) The translocation breakpoints of four cases appeared simple and balanced at the resolution used. (2) Three cases were found to have complex rearrangements some including deletions, inversions and insertions at or near one or both breakpoints. The two deletions, del(10q) and del(11q) were both de novo. (3) Three cases in which the translocations appeared balanced were found by microarray to have previously unrecognised imbalances, $\operatorname{dup}(3 p), \operatorname{del}(6 q)$ and $\operatorname{del}(18 q)$. The $\operatorname{dup}(3 p)$ was also present in the phenotypically normal father, the del(6q) was de novo and the status of the del(18q) was unresolved. We are also conducting a similar series of experiments to examine the breakpoints in ten phenotypically normal individuals carrying apparently balanced familial translocations so that the level of complexity involving the breakpoints and other genomic regions can be compared between the two groups. Our results on the de novo phenotypically abnormal patients will have a significant impact on current diagnostic investigations of patients of this type and provide an argument for the more widespread adoption of microarray analysis or other high resolution genome-wide screens for chromosome imbalance and rearrangement.

L177

\section{DNA array based analysis of chromosomes in leukaemia}

\section{B. Young ${ }^{1}$}

${ }^{1}$ Molecular Oncology Group, Medical Oncology Unit, Cancer Research UK Clinical Centre, UK

No abstract was submitted for this talk.

\section{L178}

\section{Human meiotic recombination: a high-resolution view}

C. May ${ }^{1}$, K. Holloway ${ }^{1}$, L. Kauppi ${ }^{1}$, T. Slingsby ${ }^{1}$, R. Neumann ${ }^{1}$, A. Webb ${ }^{1}$ and A. Jeffreys ${ }^{1}$

${ }^{1}$ Department of Genetics, University of Leicester, Leicester, LE1 7RH, UK. 
A detailed understanding of how meiotic recombination shapes human DNA diversity will be key in developing successful strategies for mapping common disease genes, a challenge that has been enthusiastically embraced by the research community. Global patterns of recombination can be gleaned from comparisons of physical and genetic maps, but higher resolution analysis relies on the fine-scale characterization of many recombinants over intervals of just a few kilobases. To this end, we have exploited the abundant SNP class of DNA marker and have developed PCR-based strategies to detect de novo recombinant molecules directly from batches of sperm DNA. Data from different regions of the genome indicate that crossovers tend to cluster into very narrow hotspots and that these hotspots can profoundly influence patterns of haplotype diversity within a population. Gene conversion events co-localise with the peak of crossover activity but can be more frequent and are even more tightly distributed affecting haplotypes at an extremely localised level.

References

Jeffreys et al (2000) Human Molecular Genetics 9: 725-733. Jeffreys et al (2001) Nature Genetics 29: 217-222.

May et al (2002) Nature Genetics 31: 272-275.

Kauppi et al (2003) Human Molecular Genetics 12: 33-40.

Jeffreys \& May (2004) Nature Genetics 36: 151-156.

\section{L179}

High-resolution genomic microarrays: applications for the study of chromosome biology

D. Vetrie ${ }^{1}$

${ }^{1}$ Sanger Institute, Hinxton, Cambridge, UK

No abstract was submitted for this talk.

\section{L180}

\section{Deciphering human genome function by multi-species sequence comparisons}

E. Green ${ }^{1}$ and Nisc comparative sequencing program $^{1}$

${ }^{1}$ National Human Genome Research Institute, National Institutes of Health, Bethesda, Maryland, USA
The Human Genome Project's recent completion of a high-quality sequence of the human genome represents a landmark scientific accomplishment of historic significance. It also signifies a critical transition for the field of genomics, as the focus shifts from elucidating the human genome sequence to determining the functional information it encodes. The comparison of sequences generated from different extant species has emerged as a powerful strategy for identifying functionally important genomic elements.

As a complement to ongoing efforts to sequence entire genomes, the NISC Comparative Sequencing Program is pursuing multi-species genome explorations by sequencing and analyzing the same orthologous regions in many different vertebrates. To date, we have generated over $450 \mathrm{Mb}$ of comparative sequence data from more than 30 different species. Comparative analyses of these data are revealing important insights about the patterns of sequence conservation among species. In particular, we are developing approaches for utilizing multi-species sequence comparisons to identify highly conserved non-coding sequences, which likely correspond to regulatory and other functionally important elements. In addition, we are examining the relative 'informativeness' of different speciesâ sequences for identifying such highly conserved regions.

In conjunction with ongoing and future wholegenome sequencing efforts, our studies should advance the utility of comparative sequence analyses, and make important contributions towards unraveling the functional and evolutionary complexities of the human genome.

\section{Prize Nominee Special Symposium}

\section{L181}

\section{FISHing with Locked Nucleic Acids (LNA)}

\section{A. Silahtaroglu' ${ }^{1}$ H. Pfundheller ${ }^{2}$, A. Koskin ${ }^{2}$, N. Tommerup ${ }^{1}$ and S. Kauppinen ${ }^{3}$}

\footnotetext{
${ }^{1}$ Wilhelm Johannsen Centre for Functional Genome Research, Dept. of Medical Genetics, Inst. of Medical Biochemistry and Genetics, The Panum Institute, University of Copenhagen, Denmark; ${ }^{2}$ Department of Chemistry, Exiqon, Bygstubben 9, DK-2950 Vedbaek, Denmark;
} 
${ }^{3}$ Department of Functional Genomics, Exiqon, Bygstubben 9, DK-2950 Vedbaek, Denmark

Locked Nucleic Acids (LNA) constitute a novel class of RNA analogues having an exceptionally high affinity towards complementary DNA and RNA. The ribofuranose ring in LNA is structurally constrained by a methylene bridge between its $2^{\prime}$-oxygen and the $4^{\prime}$-carbon atoms resulting in a locked $3^{\prime}$-endo conformation, thereby reducing its conformational flexibility and increasing the local organization of the phosphate backbone. LNA obeys the Watson-Crick base-pairing rules and LNA bases are furthermore linked by a phosphodiester backbone, thus allowing synthesis of chimeric LNA/DNA and LNA/RNA oligonucleotide probes using standard phosphoramidite chemistry. Finally, an important practical advantage is that LNA is fully soluble in water, which makes its handling and the experiments simple.

The development of molecular probes and image analysis has made fluorescence in situ hybridization (FISH) a powerful investigative tool. Although FISH has proved to be a useful technique in many areas, it is still a fairly time-consuming procedure with limitations in sensitivity and resolution. Thus, probes with higher affinity would potentially improve the sensitivity of the technique.

Here we describe the development of LNAsubstituted oligonucleotides as probes for fluorescence in situ hybridization on metaphase chromosomes and interphase nuclei. We have used several different human-specific repeats such as the classical satellite-2 repeats, telomere repeats and alpha satellite repeats as targets and demonstrated that LNA oligos are excellent probes for FISH, combining high binding affinity with short hybridization time and even with the ability to hybridize without prior thermal denaturation of the template.

\section{L182}

Imaging of the human metaphase chormosomes by atomic force microscopy in liquid

O. Hoshi ${ }^{1}$, R. Owen ${ }^{2}$, M. Miles ${ }^{2}$ and

T. Ushiki ${ }^{1}$
${ }^{1}$ Division of Microscopic Anatomy and Bio-imaging, Department of Cellular Function, Niigata University Graduate School of Medical and Dental Science, Niigata, Japan; ${ }^{2}$ H.H.Wills Physics Laboratory, University of Bristol, Bristol, UK

The high-order structure of human metaphase chromosomes is still controversial, due to the technical difficulty of its high resolution analysis in a natural condition. Atomic force microscopy (AFM), which has been recently applied to the biological field, has the advantage in obtaining sample images in various (vacuum, air and liquid) environments at high spatial resolution. Thus, the aim of the present study is to clarify the native conformation of the human metaphase chromosomes by observing them in a liquid environment with an AFM. Metaphase chromosomes were harvested from human lymphocytes, suspended in a methanol-acetic acid solution and spread on a clean glass slide. The chromosomes 1 and 2 were observed using an intermittent contact mode in a phosphate-buffered saline solution. Symmetrical alternating ridges and grooves were present on the surface of the sister chromatids in individual chromosomes. The number and arrangement of the ridges and grooves were specific to the type of the chromosome. We also observed the change in structure of chromosome caused by the quinacrine mustard treatment (Q-banding) using AFM in liquid. A comparison of images of the same chromosome before and after the staining treatment showed that the basic structure of the chromosome was not changed, except that the height difference between the ridges and grooves was increased. These results show that the AFM observation in liquid is useful for analysing the chromosome structure and its change in relation to the effect of various staining treatments.

\section{L183}

Strand-specific fluorescence in situ hybridization: CO-FISH

S. Bailey ${ }^{1}$, M. Cornforth ${ }^{2}$ and

E. Goodwin ${ }^{3}$ 
${ }^{1}$ Colorado State University, Fort Collins, CO, USA; ${ }^{2}$ University of Texas Medical Branch, Galveston, TX, USA; ${ }^{3}$ Los Alamos National Laboratory, Los Alamos, NM, USA

The ability to prepare single-stranded chromosomal target DNA allows innovative uses of FISH technology for studies of chromosome organization. Standard FISH methodologies require functionally single-stranded DNAs in order to facilitate hybridization between the probe and the complementary chromosomal target sequence. This usually involves denaturation of doublestranded probe and target DNAs to induce temporary separation of the DNA strands. The strand-specific CO-FISH (Chromosome Orientation-FISH) method involves selective removal of newly replicated strands from the DNA helixes within metaphase chromosomes. The result is single-stranded target DNA. Single-stranded probes can then be hybridized to the single-stranded chromosomal target DNA, resulting in strand-specific hybridization - revealing a wealth of information previously unattainable at the cytogenetic level.

Mammalian telomeric DNA consists of tandem repeats of the (TTAGGG) sequence, oriented $5^{\prime}$ to- $3^{\prime}$ towards the end of the chromosome. Based on this conserved structural organization, COFISH with a telomere probe can be used to determine the orientation of DNA sequences along chromosomes. Applications of the CO-FISH approach will be presented and include detection of pericentric inversions and isochromosomes, discrimination between leading vs. lagging strand telomeres, identification of dysfunctional telomere-double strand break fusions, a combined SKY-CO-FISH method, as well as revelation of replication timing of mammalian telomeres via an approach we term ReD-FISH.

\section{L184}

Species divergence in Asparagales is associated with the evolution of novel types of telomeres

E. Sykorova ${ }^{1}$, K. Lim², Z. Kunicka ${ }^{3}$,

G. Rotkova ${ }^{3}$, M. Chase $^{4}$, M. Bennett ${ }^{4}$, A. Leitch ${ }^{2}$ and J. Fajkus ${ }^{1}$
${ }^{1}$ Institute of Biophysics, Czech Academy of Sciences, Brno, Czech Republic;

${ }^{2}$ School of Biological Sciences, Queen Mary University of London, UK; ${ }^{3}$ Department of Functional Genomics and Proteomics, Masaryk University Brno, Czech Republic; ${ }^{4}$ Jordell Laboratory, Royal Botanic Gardens, Kew, Richmond, UK

Analysis of telomeres within the plant order Asparagales has shown a remarkable polymorphism of telomere DNA sequences. In association with a phylogenetic tree of Asparagales, these results show original 'typical' plant telomeric repeats [TTTAGGG]n have been partially or fully replaced by the human-type telomeric sequence [TTAGGG]n in a distinct clade within Asparagales. Correspondingly, telomerases of these species synthesise human repeats with a high error rate in vitro. Further, even more profound change has occurred within this clade in Alliaceae resulting in the absence of any known telomeric minisatellites and corresponding telomerase activity. Telomerase mutation(s) responsible for the switch in telomeric DNA sequence could be expected to have an impact on interactions of telomere-binding proteins involved in telomere structure and function. However, our results show that nuclear protein extracts from plants with human-like telomeres form specific complexes with both typical plant and human telomeric DNA sequence.

This work has been supported by the Leverhulme Trust, GACR (204/02/0027) and the Institutional support (MSM143100008 and AV0Z5004920).

\section{L185}

The dynamic centromere: chromosome evolution in marsupials

\section{R. O'Neill'}

${ }^{1}$ Dept. Molecular and Cell Biology, University of Connecticut, U-2131, Storrs CT 06269, USA

Studies of chromosome evolution have focused heavily on the evolution of conserved syntenic, gene-rich domains. It is obvious, however, that the centromere plays an equally important role in chromosome evolution, through its involvement in fissions, centric fusions, translocations, inver- 
sions and centric shifts. It is unclear how the centromere, either as a functioning unit of the chromosome or as a DNA sequence motif, has been involved in these processes. Macropodine marsupials (kangaroos and wallabies) offer unique insights into current theories expositing centromere emergence during karyotypic diversification and speciation. Tracing the phylogentic distribution of centromeric sequences, including tandem repeats and retroelements, within this group of mammals indicates these sequences have played an important role in chromosome evolution through amplifications, segmental duplications, fissions and fusions. Hybrids between different kangaroo species provide evidence that the centromere is unstable within this group of mammals and is involved in a large number of chromosome aberrations, including the appearance of knobs. A better understanding of the genetic and epigenetic factors that define centromeres and how centromeres may mediate changes in chromosome architecture are critical not only to our understanding of basic cellular functioning but also to our understanding of the process of speciation.

\section{L186}

\section{Proteomic analysis of human metaphase chromosomes}

S. Uchiyama ${ }^{1}$, S. Kobayashi ${ }^{1}$, H. Takata $^{1}$, T. Ishihara ${ }^{1}$, T. Sone ${ }^{2}$, T. Nirasawa ${ }^{3}$, T. Takao ${ }^{4}$, S. Matsunaga ${ }^{1}$ and K. Fukui ${ }^{1}$

${ }^{1}$ Department of Biotechnology, Graduate School of Engineering, Osaka University; ${ }^{2}$ Institute for Microbiological Diseases, Osaka University;

${ }^{3}$ Bruker Daltonics, K. K.; ${ }^{4}$ Institute for Protein Research, Osaka University, Japan

Formation of mitotic chromosome is needed to ensure the equal partition of the semi-conservatively replicated genomic DNA into daughter cells during mitosis. Despite more than a century of research on chromosome structure, it still remains poorly understood.

We report the comprehensive list of proteins involved in isolated human metaphase chromosomes for the elucidation of chromosome higher order structures. Proteins separated by one- dimensional (1-D) SDS-PAGE and isoelectric focusing and radical free and highly reduced twodimensional electrophoreses were identified to draw up a list of constituent proteins with quantitative information. Image analysis of 1-D SDSPAGE pattern indicated most abundant proteins were histones, of which population exceeds $60 \%$ of total amount of chromosomal proteins. A total of 158 different proteins were identified from chromosomes isolated with polyamine procedure followed by sucrose density gradient centrifugation. Classification according to known localization indicated that $32 \%$ (51 proteins) of the number of the identified proteins belong to nuclear ones, of which 25 proteins are reported to localize to mitotic chromosomes. The isolated chromosomes included substantial number of nucleolar proteins, indicating that these chromosomes contain not only chromosomal proteins. In addition to chromatin binding proteins related to transcription, topoisomerase II alpha and subunits of condensin complex were identified, although the amounts of these chromosome associated proteins are rather low. Finally, the identified proteins were classified into four groups based on the behavior during the purification and known data during mitosis. Present catalogue would provide essential data for understanding metaphase chromosomes based on the constituent proteins.

\section{L187}

\section{The fate of primary aneuploid cells during mouse embryo development}

\section{Lightfoot ${ }^{1}$ and C. Höög ${ }^{1}$}

${ }^{1}$ Dept. of Cell and Molecular Biology, Karolinska Institute, Sweden

The absence of synaptonemal complex protein 3 (SYCP3) incites chromosomal nondisjunctions during the first meiotic division in murine female germ cells, resulting in the formation of primary aneuploid haploid oocytes. This model system is unique in that it can reliably produce large numbers of primary aneuploid oocytes. We have studied the fate of fertilized mouse embryos resulting from these abnormal cells and find that primary aneuploid embryos can implant into the female uterine tissue and initiate gastrulation 
(E6.5). At E7.0, however, the embryonic cells begin to display abnormal morphological signs. Spherical cellular blebbings originating from the inner wall of the developing embryo are seen aggregating into the center of the proamniotic cavity, as they become dissociated from the ectodermic cellular layer. The inner cellular layers of the embryo then degrade and the embryo forms an indiscernible mass by E8.5. The embryo degradation process follows a singular pattern, initially seen within the embryonic ectoderm, along the lateral median of the embryo, and progressing outward to the distal poles, and latter the surrounding deciduea. Concurrent with the morphological changes in the dying embryos, nuclear abnormalities are observed showing that loss of embryo viability is caused by the activation of a spatially and temporally controlled apoptotic mechanism. This apoptotic response to systemic primary aneuploidy is p53-independent and does not result from a failure to progress through mitosis. We find that primary aneuploid embryos display a different developmental fate than what has been observed for secondary (Robertsonian) or tertiary (partial, segmental) aneuploid conceptus.

\section{L188}

\section{Characterisation of all human male synaptonemal complexes with subtelomere multiplex-FISH}

M. Codina-Pascual' ${ }^{1}$, J. Kraus², M. Speicher ${ }^{2}$, M. Oliver-bonet ${ }^{1}$, V. Murcia ${ }^{1}$, J. Sarquella ${ }^{3}$, J. Egozcue ${ }^{1}$, J. Navarro ${ }^{1}$ and J. Benet ${ }^{1}$

${ }^{1}$ Unitat Biologia, Facultat Medicina, Dept. Biologia Celular, Fisiologia i Immunologia, UAB, Bellaterra, Spain; ${ }^{2}$ Institut für Humangenetik, Technische Universität München and GSF Neuherberg, Germany; ${ }^{3}$ Unitat de Reproduccion Humana i Diagnostic Gentic, Clinica Girona, Girona, Spain

During meiotic prophase I, homologous chromosomes synapse and recombine in pachytene stage. Both events are of vital importance for the success of meiosis. When homologous chromosomes synapse a proteinacious structure called synaptonemal complex (SC) appears along the pairing axis and meiotic recombination is taking place. The existence of immunolabelling techniques for SC proteins (SCP1, SCP2 and SCP3) and for DNA mismatch repair proteins present in late recombination nodules (MLH1) allow analyses of both synapsis and meiotic recombination in the gametocyte I. The creation of recombination maps for each chromosome and the analysis of each bivalent synapsis is possible by in situ hybridisation methods because chromatin is preserved after cell fixation for immunoanalysis. In this work we apply the seven-fluorochrome subtelomere-specific FISH assay (Fauth et al., 2001, Hum Genet. 109:576) to human male meiotic cells previously labelled by immunofluorescence (SCP1, SCP3, MLH1, CENP) to assess its utility for human SC karyotyping. This FISH method consists of microdissected subtelomeric probes labelled combinatorially with seven different fluorochromes. Results prove the usefulness of this FISH method (stM-FISH) for the identification of all human SC. Furthermore, by labelling subtelomeric regions this one-single-step method enables the characterisation of interstitial and terminal SC fragments and SC delineation even if superposition is present in pachytene spreads.

\section{L189}

\section{B chromosomes alter the metaphase checkpoint in grasshopper meiocytes}

\author{
B. Herron ${ }^{1}$, S. Martin-Iluesma ${ }^{2}$, P. Arana ${ }^{3}$ \\ and D. Wise ${ }^{1}$ \\ ${ }^{1}$ Department of Biological Sciences, Mississippi \\ State University, Mississippi State, MS 39762, \\ USA; ${ }^{2}$ Max Planck Institut fur Biochemie; \\ ${ }^{3}$ Universidad Complutense, Madrid, Spain
}

The B chromosome polymorphism in Spanish populations of the grasshopper, Eyprepocnemis plorans (Charpentier) is ancient and widespread. Meiocytes containing B chromosomes were analyzed in our laboratory using the $3 \mathrm{~F} 3 / 2$ monoclonal antibody, which binds to a kinetochore phosphoepitope whose degree of phosphorylation is sensitive to tension applied to the kinetochore. Further, the tension created by the spindle at metaphase controls a checkpoint (the metaphase checkpoint) that allows the cell to begin ana- 
phase when all chromosomes are aligned at the metaphase plate. Fluorescence patterns of the $3 \mathrm{~F} 3 / 2$ phosphoepitope in cells containing B chromosomes were determined using confocal laser scanning microscopy. The phosphorylation pattern of kinetochores in these cells was shown to be different from that of cells without Bs. This suggests that the metaphase checkpoint has been modified in some way. We propose that B chromosomes in these grasshopper populations may have survived during evolution due to an alteration of the metaphase checkpoint, making it more permissive to the presence of misaligned chromosomes.

\section{L190}

\section{Human artificial chromosome expression marker}

\author{
A. Laner $^{3}$, S. Christan ${ }^{2}$, T. Schwarz ${ }^{1}$ \\ and D. Schindelhauer ${ }^{2}$ \\ ${ }^{1}$ Medizinische Genetik, Kinderpoliklinik LMU, \\ Munich, Germany; ${ }^{2}$ Livestock Biotechnology, \\ Life Sciences Center Weihenstephan, TU-Munich, \\ Freising, Germany; ${ }^{3}$ Medizinisch Genetisches \\ Zentrum, Munich, Germany
}

Human artificial chromosomes are generated using transfer of telomerized PAC constructs containing alpha satellite DNA of various human chromosomes. To monitor which cells obtained constructs and subsequently form stable clones under blasticidin $\mathrm{S}$ selection, a CMV/EGFP expression marker was inserted into a chr 5 alpha satellite construct $(142 \mathrm{~kb})$. Lipofection into HT1080 cells resulted in a subportion of bright green cells under the microscope at day 1 . Areas containing such early green cells were marked and plates monitored over 2 weeks. In only one out of 41 marked areas (from 2 experiments) a viable clone developed. In the remaining 40 areas, the green cells ceased growth at the $1-8$ cell stage (similar results were observed in sections taped to exclude light damage). In contrast, outside of the marked areas, 16 stable clones have formed, which became uniformly green not before day 4-6. FISH analysis of isolated clonal lines demonstrated human artificial chromosome formation and stable segregation without integration. We conclude that transient expression of an EGFP marker is not suitable to identify transfected cells, which will subsequently form viable clones. One explanation could be that the high copy number required to consistently detect transient EGFP-marker expression [1] impairs viability and clone formation.

[1] Schindelhauer D., Laner, A. (2002) Visible transient expression of EGFP requires intranuclear injection of large copy numbers. Gene Ther. 9: 727-30.

\section{L191}

Fundamental structural unit of the chromosome identified by atomic force microscopy

\author{
K. Takeyasu' ${ }^{1}$, J. Kim ${ }^{1}$, M. Yokokawa ${ }^{1}$ \\ and S. Yoshimura ${ }^{1}$ \\ ${ }^{1}$ Kyoto University Graduate School of Biostudies, \\ Japan
}

The proper function of the genome largely depends on the higher-order architecture of the chromosome. Nano-technology is essential to address questions regarding the structural basis for such macromolecular dynamics [1-3]. Because of its applicability to biological specimen (nonconductors) under physiological conditions, atomic force microscopy (AFM) was employed for the structural analyses. After HeLa cells on a cover glass were successively treated with a detergent and a high-salt solution to remove the cytoplasmic and nucleoplasmic materials, the interphase chromosome was found to be composed of a granular unit of $\sim 80 \mathrm{~nm}$ in diameter, forming a fiber of $\sim 80 \mathrm{~nm}$ width. When this onsubstrate procedure was applied to Escherichia coli, the nucleoid was found to consist of a fundamental fibrous structure with a diameter of $\sim 80 \mathrm{~nm}$. The nucleoid dynamically changed its structure during the cell growth; In addition to this ' $80-\mathrm{nm}$ fiber', a thinner '40-nm fiber' and a higher-order structure of 'loop' were identified in the $\log$ phase nucleoid. However, in the later growth phases, the nucleoid turned into a 'coral reef structure' that also possessed the 80 -nm fiber 
units, and, finally, into a 'tightly compacted nucleoid' that was stable under a mild lysis buffer. Mutant analysis demonstrated that these tight compactions of the nucleoid required a protein, Dps. From these, we propose a structural model that assumes a fundamental mechanism of chromosome packing is common in both prokaryotes and eukaryotes.

1. Curr. Bio., 12: 508-512; 2. Proc. Nat'l Acad. Sci. USA, 97 : 7266-7271; 3. Biochemistry, 39: 9139-9145. 



\section{Author Index}

L denotes Lecture abstract; PO denotes Poster abstract.

Abdulrazzak, H. L112, L118, PO:08:17, PO:08:19

Abe, S. L125, PO:08:71

Abeydeera, L. L11

Abgrall, J.-F. PO:08:85, PO:08:91

Abramowicz, M. L46

Acevedo, M. PO:06:65

Adega, F. PO:06:01

Advani, S. PO:08:90

Affara, N. L101

Agarwal, M. PO:08:90

Agata, K. L65, PO:06:52

Aguilera, M. PO:06:54

Ahlgren, T. L172

Ahn, N. PO:06:06

Al-Awadi, S. A. PO:08:46

Albano, F. PO:08:84, PO:08:94

Albiez, H. PO:06:66, PO:06:69

Albrecht, B. L146

Ali, H. L88

Alkhimova, O. L81, PO:06:56

Allain, D. L10

Alonso-Gonzalez, L. PO:06:22

Altinkut, A. PO:06:72

Amariglio, N. L157

Anamthawat-Jonsson, K. PO:06:73, PO:08:38, PO:08:53

Ando, J. L65, PO:06:53

Andolina, M. PO:08:39, PO:08:45

Anelli, L. PO:08:84, PO:08:94

Ansari, R. PO:08:54

Anton, E. PO:08:23

Apisitwanich, S. PO:08:78

Arana, P. L189

Aranyavalai, V. PO:06:02

Arbour, L. L126

Arnaud, B. PO:08:85

Arneric, M. L68

Arrieta, I. PO:08:50, PO:08:52

Assenberg, M. PO:08:20

Astakhova, N. PO:06:64

Athalye, A. PO:08:72, PO:08:90

Attarian, H. PO:08:59

Aulard, S. PO:06:50

Aveline-Wolf, L. PO:06:06

Ayabe, F. PO:08:18

Ayenehchi, S. PO:08:49

Azuma, T. PO:06:08

Babcock, J. L117

Bacher, C. P. PO:06:67

Bageri, N. PO:08:73

Baharizadeh, S. PO:08:59

Bailey, S. L183

Bair, E. L169
Balanya, J. L129

Balmus, G. PO:06:04

Balu, S. PO:08:10

Bandkar, V. PO:08:72, PO:08:90

Bang, J. PO:06:80, PO:06:82

Banzakour, S. PO:08:85, PO:08:91

Baroni, R. M. PO:08:31

Barrett, J. L130

Bartos, J. L79, PO:06:83, PO:06:92

Bártová, E. PO:06:70

Battaglia, A. PO:08:76

Battini, R. PO:08:76

Battle-Morera, L. L99

Baulcombe, D. L75

Behjati, M. PO:08:61

Belil, I. PO:06:34

Bellovits, O. PO:08:37

Belmont, A. L34, L37

Belyayev, A. L80, L85, PO:06:72

Benatti Jr., R. PO:08:31

Benet, J. L54, L188, PO:06:34

Bennett, M. L184, PO:06:45, PO:06:46

Bentz, M. L166

Bermúdez, M. L54, PO:06:34

Bernardino-Sgherri, J. PO:08:25

Bertaud, M. L10

Berthou, C. PO:08:85, PO:08:91

Bertini, V. PO:08:76

Betti, M. PO:06:61

Beverloo, B. L173, L174

Bhardwaj, R. PO:06:79

Bheemineni, S. PO:06:15, PO:06:33

Bickel, S. L149

Bickmore, W. L31

Bidau, C. L63

Bird, A. L71

Bishehsari, F. PO:08:54

Bisoni, L. L99

Blanco, J. PO:08:23, PO:08:34

Bleuyard, J. PO:08:24

Bonduelle, M. L49

Bongers, E. L159

Bonifacio, S. PO:06:35

Bonne, G. L30

Boobis, I. PO:08:74

Boudry, P. PO:06:13, PO:08:65

Bouilly, K. PO:08:65

Bourquard, P. PO:08:85, PO:08:91

Bourrouillou, G. L46

Böwering, K. L146

Boyle, S. L31

Bozzini, G. PO:06:04

Brack-Werner, R. PO:06:69

Bradbury, M. PO:06:63 
Branco, M. L39

Brenton, J. L74

Bretherick, K. L152

Bridger, J. L11, L30, L111

Britton-Davidian, J. L104

Brodie, S. PO:08:86

Brown, C. L97, L152

Brown, J. PO:08:55

Brown, L. L100

Brown, W. L116

Bruyere, H. L126

Buijs, A. PO:08:87

Buiting, K. PO:06:71

Bujdosó, G. PO:08:37

Bullinger, L. L169

Buquicchio, C. PO:08:84

Burford, D. L176

Burgoyne, L. PO:06:03

Burgoyne, P. L101, PO:08:35

Burn, J. L01

Burt, D. L09, L56

Bystricky, K. L35

Cabuy, E. L70

Camats, N. PO:06:57

Campiranon, A. PO:08:60

Campos, L. PO:08:92

Canham, P. L26

Cao, A. PO:06:60

Carniani, S. PO:06:35

Carpenter, A. PO:06:22

Carpentier, S. PO:08:36

Carrera, M. PO:08:75

Carrillo, J. A. PO:06:27

Carter, N. L31, L175, L176

Carvalho, A. L18

Carvalho, B. PO:08:15, PO:08:16

Ceccarelli, M. PO:06:23

Cesari-Weimar, F. L74

Céspedes, W. L129

Chadwick, B. L117

Chaminade, N. PO:06:50

Chantry-Darmon, C. L10

Chase, M. L184, PO:06:62

Chaumeil, J. PO:06:68

Chaves, R. PO:06:01, PO:06:13, PO:08:65

Cheeseman, I. L19

Chetan, K. G. PO:08:10

Cheung, W. L112, L118, PO:08:19

Chiaroni, J. L102

Chicheportiche, A. PO:08:25

Chokchaichamnankit, P. PO:08:38

Choo, A. K. L07, L26, L27

Chow, J. L97

Christan, S. L190

Christopikou, D. L50

Chudzinska, E. PO:06:74

Chulalaksananukul, W. PO:06:02, PO:08:38

Chung, W. PO:08:17
Chuzhanova, N. L60

Ciampi, R. PO:06:61

Cianchetti, C. PO:06:60

Cihalikova, J. L79, PO:06:83, PO:06:92

Cioni, G. PO:08:76

Cirakoglu, A. PO:08:56, PO:08:77

Cisel, B. PO:08:88

Clegg, S. L176

Clemente, E. L101

Clouston, H. L51

Cloutier, S. PO:06:26

Codina-Pascual, M. L188

Coe, B. L139

Cohen, P. L123

Collignon, P. L102

Colls, P. L54

Colovic, M. PO:08:39, PO:08:45

Conn, C. L47

Constantinou, M. PO:06:16, PO:08:06

Cook, P. PO:06:38

Cooper, D. L60

Cooper, J. L67

Coppola, G. PO:06:04, PO:06:04

Cornforth, M. L183

Cornillon, J. PO:08:92

Corredor, E. PO:08:26, PO:08:27

Cowan, R. S . PO:06:38

Cox, H. L111

Craig, J. L26, L27

Cremer, C. L161, PO:06:32

Cremer, M. PO:06:32, PO:06:69

Cremer, T. L33, L42, L76, PO:06:66, PO:06:69, PO:06:71

Criado, B. PO:08:50, PO:08:52

Crisponi, G. PO:06:60

Crolla, J. L176

Crooijmans, R. L09

Csonka, E. L158, PO:08:37

Culic, V. PO:08:66

Dafhnis-Calas, F. L116

Dahm, P. L132

Dalakiouridou, M. L50

Dame, R. L93, PO:08:01

Daniely, M. L157

David, P. PO:08:30

De Braekeleer, M. PO:08:85, PO:08:91

De Carli, L. L36

De Jong, H. L88

De la Herrán, R. PO:08:08, PO:08:09

de Leeuw, N. L159

de Paepe, A. L142

de Rochambeau, H. L10

de Vries, B. L133

Dechyeva, D. PO:06:75

Dedovic-Tanic, N. L15

Defeu Soufo, H. L95

Dekker, C. L93

Deladesse, E. PO:08:92

De-Lange, T. $\mathbf{L 6 6}$ 
Delhanty, J. L47

Demidov, D. $\mathbf{L 8 7}$

Deng, C. PO:08:35

Dennis, N. L176

Derjusheva, S. L13, PO:06:37, PO:08:07

Desai, A. L19

Deviren, A. PO:08:77

Devroey, P. L49

Dhumal, S. PO:08:72, PO:08:90

Di Berardino, D. PO:06:04

Di Meo, G. PO:06:04

Diaz, A. PO:06:12

Dickson, G. L113

Dimitrijevic, B. L15

Ditchfield, C. L22

Dmoszynska, A. PO:08:88, PO:08:89

Döhner, H. L169

Döhner, K. L169

Dolezel, J. L79, PO:06:56, PO:06:83, PO:06:92

Dombrowski, C. PO:06:75

Donev, R. L43

Donnison, I. PO:06:78

Dordari, S. PO:06:05

Douet-Guilbert, N. PO:08:85

Draper, J. PO:06:77, PO:06:78

Duprez, L. L46, PO:08:40

Duran, A. PO:08:51

Dutrillaux, B. PO:08:25

Dyban, A. PO:06:20

Dyer, M. S. L167

\section{Earle, E. L26}

Earnshaw, W. L18, PO:06:22

Egozcue, J. L188, PO:06:34, PO:06:57, PO:08:23, PO:08:32, PO:08:33, PO:08:34

Egozcue, S. PO:08:34

Egriboz, A. PO:08:51

Eijpe, M. L122

Eils, R. L76, L164, PO:06:67

Eleveld, M. L154

Ellis, J. A. L111

Ellis, P. L101

El-Mogharbel, N. L106

Emma, D. PO:08:30

Emrick, M. PO:06:06

Endo, T. PO:06:92

Engelbrecht, S. PO:08:03, PO:08:55

Enne, G. PO:06:04

Enukashvily, N. L43

Erhardt, S. L74

Errington, J. L94

Escalona, A. PO:08:75

Evans, C. L133

Fadaei, S. L77

Faed, M. L47

Fairclough, H. L61

Fajkus, J. L184, PO:06:62

Fang, J. L133
Farahman, H. PO:08:73

Faridi, N. PO:06:76

Farr, C. L06, PO:06:22, PO:08:22

Fay, M. PO:06:38

Fedoryshyn, Z. PO:08:67

Feenstra, I. L133

Feingold, E. L151

Ferguson-Smith, M. L09, L41, L55, L61, L106

Fernandez, R. PO:08:68

Ferreira, I. L17, PO:06:07

Fiegler, H. L31

Field, J. K. PO:06:25

Figgitt, M. L30

Filatov, D. L103

Filip, A. PO:08:88, PO:08:89

Filipe, S. L92

Fillon, V. L09

Finsterle, J. L161

Fioretos, T. L172

Flandrin, P. PO:08:92

Fleming, E. L158

Flores, P. PO:08:50, PO:08:52

Fogel, M. PO:08:02

Fontdevila, A. L129

Foresti, F. L17, PO:06:07

Förstemann, K. L68

Foster, H. L11, L30

Fragouli, E. L47

Fransz, P. L88

Frederic, V. L104

Frigyesi, A. L145

Fröhling, S. L169

Fuchs, J. L45, L64, PO:06:28

Fujiwara, A. PO:06:53

Fukagawa, T. L23, PO:06:22

Fukova, I. L105, PO:08:04, PO:08:13

Fukui, K. L14, L82, L83, L186, PO:06:08, PO:06:10

Fukushi, D. PO:08:81

Furlong, R. L101

Fuster, C. PO:08:75

Gaginskaya, E. L13, L160, PO:06:37, PO:08:07

Gair, J. L126

Galetti Jr., P. M. L17

Galkina, S. PO:06:31

Gallardo, M. L63

Gallego, M. PO:06:58, PO:08:24

Gamerdinger, U. PO:08:69

Gao, J. L76

Garagna, S. L44

Garcia, M. PO:06:51, PO:06:54, PO:06:57, PO:08:32, PO:08:33

Garcia, R. PO:08:32, PO:08:33

García, F. PO:06:54, PO:06:57

Gardunia, B. R. PO:06:81

Garnis, C. L139

Garrido-Ramos, M. A. PO:08:08 PO:08:09

Gasser, S. L35

Gassmann, R. L18 
Gazdar, A. L139

Gelati, M. T. PO:06:23

Gerats, T. L88

Ghadiani, M. PO:08:59

Ghasemi Barghi, M. PO:08:49

Gibbons, N. PO:08:83

Gibby, M. L130

Gifford-Garner, J. PO:08:19

Gilbert, N. L31

Giltay, J. L154

Girimaji, R. S. PO:08:10

Giselsson, D. L145

Glazko, T. L40, L136

Glazko, V. L136

Glazkov, M. PO:06:24

Glover, D. PO:08:11

Goidts, V. L60

Goodwin, E. L183

Goosen, N. L93, PO:08:01

Gordei, I. PO:08:28

Goswami, H. PO:06:39

Graumann, P. L95

Graves, J. L61

Greaves, M. L171

Green, E. L180

Greenbaum, I. L132

Greene, A. L158

Greensmith, J. PO:06:38

Gregor, T. PO:08:11

Greilhuber, J. PO:06:88

Gribble, S. L176

Griffin, D. L09, L11, L30, L50, PO:06:53, PO:08:83

Griffiths, M. L170, L171

Grimes, B. L117

Groenen, M. L09

Großmann, C. L161

Grundmann, M. L130

Gruszka-Westwood, A. L171

Grutzner, F. L61

Grützner, F. L106

Guan, X. Y. PO:08:63

Guber, A. L157

Guedes-Pinto, H. PO:06:01, PO:06:13, PO:08:65

Guichaoua, M. L102

Guillaume, M. PO:06:58

Gunel, B. PO:08:51

Guskov, E. PO:06:94

Gustavino, B. PO:06:55

Gutiérrez-Mateo, C. L54, PO:06:34

Guven, G. PO:08:77

Haas, O. A. $\mathbf{L 1 6 8}$

Habermann, F. PO:06:37

Hacihanefioglu, S. PO:08:51, PO:08:56, PO:08:57, PO:08:77

Hadlaczky, G. L144, L158, PO:08:37

Hajfathali, A. PO:08:59

Hajkova, P. L74

Hall, L. L97
Hameister, H. L60, $\mathbf{L 9 8}$

Hanaoka, K. L125

Hans, C. PO:08:40

Hanson, L. PO:06:38

Harrison, C. L170, L171

Harvard, C. PO:06:30

Hasezawa, S. L83

Hass, I. PO:06:40

Hassold, T. L148

Hasterok, R. PO:06:77, PO:06:78

Hausmann, M. L146, L161, PO:06:32

Hayes, H. L10

Heard, E. PO:06:67, PO:06:68

Hegde, J. PO:08:47, PO:08:48

Helszer, Z. PO:08:06

Hemleben, V. PO:06:47

Henzing, A. L18

Hernando, C. PO:08:75

Herr, A. $\mathbf{L 7 5}$

Herron, B. L189

Heslop-Harrison, J. S. L86, PO:06:01

Heun, P. L35

Heyting, C. L122

Higashi, T. PO:06:08

Higgs, D. L72

Hildenbrand, G. L161

Hnateiko, O. PO:08:67

Hobza, R. PO:08:05

Hochstenbach, R. L154

Hodges, C. L122

Höfler, H. L146

Höglund, M. L145

Holecova, M. PO:06:11

Holloway, K. L178

Homa, S. L50

Höög, C. L150, L187

Horsley, S. L171

Horsthemke, B. PO:06:71

Hoshi, O. L156, L182

Hoshiya, H. PO:08:18

Houben, A. L87

Hu, L. PO:08:63

Hudakova, S. PO:06:18

Hughes-Davies, L. L73

Hultén, M. L48, PO:08:29

Hunt, H. L130

Hunt, P. L120, L122

Hurtado, N. S. PO:06:09

Huxley, C. L112, L118, PO:08:17, PO:08:19, PO:08:21

Iannuzzi, L. PO:06:04

Ibraimov, A. PO:06:41, PO:06:42, PO:06:43, PO:08:41, PO:08:42, PO:08:43, PO:08:44

Imreh, S. L144, L147

Inan, M. PO:08:56, PO:08:57

Isfahani, F. PO:08:59

Ishihara, T. L186

Ishijima, J. PO:06:53

Isobe, T. $\mathbf{L 6 5}$ 
Iturra, P. PO:06:12

Iwano, M. L14

Jacobs, P. L02, L176

Jahanzad, E. PO:06:25

Jamet, D. PO:08:91

Jamilena, M. PO:08:09

Janda, J. L79

Jankovic, G. PO:08:39, PO:08:45

Javadi, H. PO:08:49

Jeffreys, A. L178

Jellen, E. PO:06:81

Jelska, A. L135

Jenkins, G. PO:06:77, PO:06:78

Jenny, W. PO:08:30

Jenuwein, T. PO:06:69

Jeong, T. PO:08:58

Jesdapatarakul, S. PO:08:60

Jessberger, R. L122

Jiang, J. L90

Jiang, R. L126

Jin, J. PO:06:87

Johansson, B. L172

Johnson, V. L22

Jones, R. L12

Jones, R. C. L106

Josette, C. L104

Jovicic, D. PO:08:70

Julia, S. PO:08:30

Juliusson, G. L172

\section{Kai, Y. L125, PO:08:71}

Kaluzewski, B. PO:06:16, PO:08:06

Kalz, L. L135

Kamimura, E. PO:06:44

Kanale, S. PO:08:47, PO:08:48

Kang, J. PO:08:58

Kaplan, T. L157

Karamisheva, T. PO:06:64

Karpen, G. L24

Katnoria, J. K. PO:06:79

Kato, N. L45, PO:06:29

Katoh, M. PO:08:18

Kauppi, L. L178

Kauppinen, S. L181

Kawabe, A. L83

Kawle, M. PO:08:72, PO:08:90

Kazmierczak, W. PO:08:82

Kazuki, Y. L125, PO:08:71

Kearney, L. L171

Kehrer-Sawatzki, H. L60, L98

Kejnovsky, E. PO:08:05

Kelleher, C. L68

Kelly, J. L28

Kempski, H. L171

Keymolen, K. L49

Khoshbin, E. PO:06:25

Kianian, S. PO:06:83

Kill, I. L30
Kim, J. L191

Kim, S.-Y. PO:06:80

Kimura, E. L156

Kimura, M. L125

King, I. PO:06:78

King, V. L62

Klatte, M. L45, L84, PO:06:28

Klinger, H. L108

Kobayashi, S. L186, PO:06:10

Kobori, T. PO:08:80

Koch, A. L42

Koch, M. PO:06:48

Koczkodaj, D. PO:08:88, PO:08:89

Köhler, D. L76

Kohn, M. L98

Kolano, B. PO:06:81

Kolia, S. PO:08:03

Koo, D. PO:06:82

Koo, S. PO:08:58

Koolen, D. L133

Koornneef, M. L88

Korniszewski, L. PO:08:06

Korver, C. M. L100

Korzeniowska, J. PO:08:88

Koskin, A. L181

Kotzamanis, G. L112, L118, PO:08:17, PO:08:19

Koukalova, B. PO:06:47

Kouzarides, T. L73

Kovacevic, R. PO:08:70

Kovarik, A. PO:06:47

Kovarova, P. L79, PO:06:83, PO:06:92

Kozubek, M. PO:06:70

Kozubek, S. PO:06:70

Krasikova, A. L13, PO:08:07

Kraus, J. L188

Kreth, G. L45, PO:06:28

Krijtenburg, P.-J. PO:08:87

Krishnamurthy, D. S. PO:08:46

Krutilina, R. PO:06:63

Kubalakova, M. L79, PO:06:83, PO:06:92

Kubickova, S. PO:08:04

Kubisiak, T. PO:06:76

Kukalev, A. PO:06:17

Kulikova, T. L13, L160

Kunicka, Z. L184

Kurganova, A. L13, PO:06:37

Kurihara, D. L83

Kuroiwa, A. L65

Kurt, H. PO:08:77

Kurth, I. L68

Kuru, D. PO:08:56, PO:08:57, PO:08:77

Kuwae, A. PO:06:10

Kuznetsova, I. PO:06:20, PO:06:59

Kwon, K. C. PO:08:58

La Starza, R. PO:08:94

Labaere, C. PO:08:36

Lachowska-Cierlik, D. PO:06:11

Ladurner, A. L165 
Lam, A. L24

Lam, E. L45, PO:06:29

Lam, N. PO:06:12

Lam, S. L139

Lam, W. L139, L152

Lamb, N. L151

Laner, A. L190

Langan, J. PO:06:25

Lange, J. L100

Langerak, A. L174

Langowski, J. L35

Lanzone, C. L63

Lapègue, S. PO:08:65

Larin-Monaco, Z. L115, PO:08:20

Lassen, C. L172

Latypov, A. PO:08:74

Laureys, g. L142

Law, S. L157

Lawrence, J. L97

Le Baccon, P. PO:06:68

Lebris, M.-J. PO:08:85, PO:08:91

Ledebur, Jr., H. L119, L158

Lee, E. L119

Lee, G. L119

Lee, W.-K. PO:06:80

Lee, Y.-K. PO:06:82

Lees, M. L133

Leese, H. L11

Leibovitch, I. L157

Leitão, A. PO:06:13, PO:08:65

Leitch, A. L184 PO:06:47, PO:06:62

Leitch, I. PO:06:38, PO:06:45, PO:06:46

Lemeunier, F. PO:06:50

Lengerova, M. PO:08:05

Lenzi, M. L123

Leonhardt, H. PO:06:66

Lermontova, I. PO:06:18

Leung, G. L91

Levy, N. L30, L102

Lichter, P. L162

Liebaers, I. L49

Liebe, B. L122, PO:08:36

Lightfoot, D. L187

Lim, J. PO:08:58

Lim, K. PO:06:47

Lim, K. Y. L184

Lim, Y. PO:06:62

Linc, G. L88

Lindenbaum, M. L158

Lingner, J. L68

Lisi, E. PO:06:35

Liso, A. PO:08:94

Liso, V. PO:08:84

Livingstone, K. L128

Loidl, J. PO:08:30

Lonergan, K. L139

Lorenz, A. PO:08:30

Lorite, P. PO:06:27

Lostao, C. PO:08:52

Loubser, M. PO:08:03
Lozano, R. PO:08:09

Lucretti, S. PO:06:83

Lukina, N. PO:06:31

Lysak, M. A. L45, L64, PO:06:28, PO:06:48

Lyusikov, O. PO:08:28

Macas, J. PO:08:05

MacAulay, C. L139

MacDonald, N. L119, L158

Mach, J. PO:06:87

Machev, N. L102

MacLeod, R. L174

Madon, P. PO:08:72, PO:08:90

Mahadevaiah, S. L101, PO:08:35

Mahdavinia, M. PO:08:54

Maleki, F. L77

Malekzadeh, R. PO:08:54

Malla, S. L116

Maluszynska, J. PO:06:81

Mancini, M. PO:08:94

Mandahl, N. PO:08:62

Manjunatha, R. K. PO:08:10

Manteuffel, R. PO:06:18

Marasek, A. PO:06:78

Marec, F. L105, PO:08:04, PO:08:13, PO:08:14

Marra, M. L139

Marseglia, G. PO:06:61

Marshall-Graves, J. L96, L106, L107

Martin-Lluesma, S. L189

Martinez Ramirez, A. L171

Martins, C. L17, PO:06:07

Marynen, P. PO:08:36

Marzin, Y. PO:08:91

Masabanda, J. L09, PO:06:53

Mascarenhas, J. L95

Mashkina, E. PO:06:94

Masumoto, H. L114

Mateos-Langerak, J. L37

Matsubara, K. PO:06:49

Matsuda, Y. L65, PO:06:44, PO:06:49, PO:06:52, PO:06:53

Matsunaga, S. L14, L83, L186, PO:06:08, PO:06:10

Mattei, M.-G. L102

Mattes, J. L76

Mauvieux, L. PO:08:92

Maxwell, A. L158

May, C. $\mathbf{L 1 7 8}$

May, D. PO:08:69

Mayya, S. PO:08:47

McCombie, H. PO:08:65

McFarlane, R. PO:08:30

McQueen, H. L99

McQueen, P. L38

Meaburn, K. L30, L111

Mehran Nejhad, R. PO:06:05

Meister, A. L45, PO:06:28, PO:06:29

Menten, B. L142

Menzel, G. PO:06:75

Merico, V. L44 
Mertens, F. PO:08:62

Mestrovic, N. PO:06:19

Meyer-Arendt, K. PO:06:06

Michiels, A. L49

Midro, A. T. $L 135$

Mieloo, H. L159, PO:06:36

Milacic, S. PO:08:70

Mileham, A. PO:08:83

Miles, M. L182

Mills, J. L47

Mills, K. L119

Mills, W. PO:06:22

Mills, W. E. PO:08:22

Minafra, L. PO:06:60

Minelli, S. PO:06:23

Minina, J. PO:06:64

Misteli, T. L38

Mitchell, M. L102

Mitelman, F. L145, L172

Miyakawa, S. PO:06:08

Mizuno, S. PO:08:07

Mogatusi, L. PO:08:03

Mohaddes, S. M. PO:08:73

MohamadzadehGhobadloo, S. L77

Mohanty, S. PO:08:72

Mohseni, J. PO:08:73

Mokros, P. PO:06:91

Monier, K. L29

Montchamp-Moreau, C. PO:06:50

Monti, M. L44

Monti-Dedieu, L. PO:06:50

Moorman, A. L171

Moosavi, H. PO:08:49

Moosavi, Z. PO:08:73

Mora, L. PO:06:51

Moralli, D. L36, PO:08:20

Moran, P. PO:06:09, PO:06:14

Morel, F. PO:08:85, PO:08:91

Morel, S. PO:08:92

Morice, P. PO:08:85, PO:08:91

Morris, W. PO:08:83

Morrow, C. L22

Movafagh, A. L77, PO:08:49, PO:08:59

Mravinac, B. PO:06:19

Müller, S. L42, L58, L60, PO:06:40

Munné, S. L54, PO:06:34

Muñoz, M. PO:06:27

Murakami, T. PO:06:52, PO:06:53

Murata, M. L89, PO:06:21, PO:06:93

Murcia, V. L188

Murdoch, A. L51

Murphy, W. L62

Muß, B. L134

Na, Y. G. PO:08:58

Nadal, N. PO:08:92

Nadalin, N. PO:06:35

Nagpal, A. PO:06:79

Naik, D. PO:08:72
Naik, N. PO:08:72

Nakagawa, K. L83

Nakano, M. L114

Nakashima, H. L114

Nakayama, S. PO:06:84

Nannetti, G. PO:06:35

Naranjo, T. PO:08:26, PO:08:27

Nasmyth, K. L121

Navajas-Pérez, R. PO:08:08, PO:08:09

Navarro, A. L102

Navarro, J. L54, L188, PO:06:34

Nelson, C. PO:06:76

Neplechova, K. PO:06:62

Nesina, I. PO:08:88

Neumann, R. L178

Neusser, M. L42

Nevo, E. L80, L85, PO:06:72

Newbold, R. L110, L111

Nicodemo, D. PO:06:04

Niessen, S. L19

Nirasawa, T. L186

NISC Comparative Sequencing, L180

Nishida-Umehara, C. L65, PO:06:44, PO:06:53

Noom, M. PO:08:01

Noori Daloii, M. PO:06:25

Norikane, S. PO:08:18

Nourmohammadi, Z. PO:06:85

Nucaro, A. L. PO:06:60

Nutini, A. PO:06:61

Obado, S. L28

O'Brien, P. L09, L41, L61, L106

O'Brien, S. L57, L62

Oda, M. PO:06:08

Oegema, K. L19

Oei, S.-L. PO:06:63

Ogur, G. L49

Ohtani, T. PO:08:80, PO:08:81

Ohzeki, J.-I. L114

Okita, C. L125, PO:08:18, PO:08:71

Olaru, D. PO:08:92

Olejek, A. PO:08:82

Oliveira, C. L17, PO:06:07

Oliver-Bonet, M. L188

O’Neill, R. L185

Ortega, B. PO:08:50

Ortiz, E. PO:08:52

Oshimura, M. L109, L125, PO:08:18, PO:08:71

Owen, R. L182

Ozdemir, N. PO:06:26

Ozyurt, E. PO:08:56, PO:08:57

Pacchierotti, F. PO:06:55

Padma, S. PO:08:10

Page, D. L100

Pagenstecher, C. L134

Palomeque, T. PO:06:27

Pålsson, E. PO:08:62

Panagopoulos, I. PO:08:62 
Pannunzio, A. PO:08:84

Parada, L. L38

Parekh, S. PO:08:90

Parikh, F. PO:08:72, PO:08:90

Park, J. W. PO:08:58

Parker, J. L59

Parriego, M. PO:08:23

Parrini, D. PO:06:61

Parris, C. L110

Pasantes, J. PO:06:09

Pasantes, J.-J. PO:06:14

Pasaro, E. PO:08:68

Pascale, C. $\mathbf{L 1 0 4}$

Pastwinska, A. PO:08:93

Patel, S. L25

Patkin, E. L78

Paulis, M. L36

Paulsson, K. L172

Pearks Wilkerson, A. L62

Pecinka, A. L45, L64, L84, PO:06:28, PO:06:29, PO:06:48

Pecon-Slattery, J. L62

Pena, B. L10

Peñagarikano, O. PO:08:50, PO:08:52

Percipalle, P. PO:06:17

Perez, C. L119, L158

Perez-Garcia, C. PO:06:14

Perez-Luz, S. L112, PO:08:21

Perkins, E. L158

Peterlin, B. L53, PO:08:12

Peters, A. L124, PO:06:69

Peters, J. L88

Petitpierre, E. PO:06:11

Peyachoknagul, S. PO:08:78, PO:08:79

Pezer, Z. PO:06:19

Pfleghaar, K. PO:06:69

Pfundheller, H. L181

Pichler, S. PO:08:11

Pichon, B. L46, PO:08:40

Pienkowska-Grela, B. PO:08:93

Pierce, K. PO:06:06

Pierozzi, N. PO:06:86, PO:08:31

Pinter, I. L130

Pinto Leite, R. PO:08:15, PO:08:16

Pinto Maglio, C. A. F. PO:06:86, PO:08:31

Pirzadeh, Z. PO:08:49

Platzer, M. L60

Plohl, M. PO:06:19

Plowas, I. PO:06:16, PO:08:06

Podgornaya, O. L43, PO:06:17, PO:06:20, PO:06:59

Poeaim, S. PO:08:60

Pollack, J. L169

Pombo, A. L39

Pommer, C. PO:06:86

Ponsà, M. PO:06:51, PO:06:54

Poot, M. L154

Porter, A. PO:06:22

Possoz, C. L92

Poulsen, T. L174

Preuss, D. PO:06:87
Prigmore, E. L176

Probst, A. PO:06:29

Puizina, J. PO:06:91

Raimondi, E. L36

Rajcan-Separovic, E. PO:06:30

Rao, N. PO:08:86

Rapalini, E. PO:08:76

Rapp, A. L161

Raskina, O. L80, L85, PO:06:72

Rechavi, G. L157

Reckviashvili, N. PO:08:64

Redi, C. A. L44

Reichart, M. L157

Ren, X. PO:08:18

Rens, W. L41, L61, L106

Repping, S. L100

Repucci, D. PO:06:03

Rerkamnuaychoke, B. PO:08:60

Resing, K. PO:06:06

Revenkova, E. L122

Ribeiro, E. PO:08:15, PO:08:16

Rieseberg, L. L128

Riha, K. PO:06:91

Risk, J. PO:06:25

Rizzoni, M. PO:06:55

Roberti, M. G. PO:08:84

Robinson, W. L126, L152

Robles, P. PO:08:32, PO:08:33

Rocchi, M. L172, PO:08:62, PO:08:84, PO:08:94

Rodionov, A. PO:06:31

Rodríguez-Criado, G. PO:08:75

Rogel-Gaillard, C. L10

Roig, I. PO:08:32, PO:08:33

Rona, R. L157

Rosek, M. PO:06:11

Ross, F. L170, L171

Rossino, R. PO:06:60

Rotkova, G. L184

Rowell, D. L131, L137

Roy, S. PO:08:10

Rozen, S. L100

Rubtsov, N. PO:06:64

Ruchaud, S. L18

Rudd, M. L117

Rudgers, G. PO:06:87

Ruiz Rejón, C. PO:08:08, PO:08:09

Ruiz Rejón, M. PO:08:08, PO:08:09

Ruiz-Herrera, A. PO:06:54, PO:06:57

Rumsey, F. L130

Rupps, R. L126

Russell, S. L130

Rygier, J. PO:08:93

Sabapara, R. PO:06:15

Safar, J. L79

Saglio, G. PO:08:94

Sahara, K. L16, L105, PO:08:14

Saifitdinova, A. L13, L160, PO:08:07 
Saito, T. L65

Salehi, M. PO:08:61

Salehi, R. PO:08:61

Säll, T. L145

Samoylova, L. PO:08:74

Sanchez, A. PO:06:52

Sánchez-García, J. L54, PO:06:34

Sandig, C. L60

Sandstrom, R. L176

Santalo, J. PO:08:23

Santos, J. L63

Santos, M. L129, PO:08:75

Santos, S. PO:06:13

Sarquella, J. L188

Sarrate, Z. PO:08:23, PO:08:34

Sarri, V. PO:06:23

Satija, C. K. PO:06:90

Sato, H. L89

Saunders, S. L73

Saut, N. L102

Sauter, G. L163

Sbalquiero, I. PO:06:40

Scascitelli, M. PO:06:55

Schermelleh, L. PO:06:66

Schertan, H. PO:08:33

Scherthan, H. L122, PO:08:36

Schindelhauer, D. L190

Schinzel, A. L133

Schlenk, R. L169

Schlesier, B. PO:06:18

Schmidt, R. L64

Schmidt, T. PO:06:75

Schmitt, E. L161

Schneeweiss, G. M. PO:06:88

Schneeweiss, H. L59, PO:06:88

Schneider, H. L130

Schober, H. L35

Schoenmakers, E. PO:08:36

Schubert, I. L45, L64, L84, L88, PO:06:18, PO:06:28, PO:06:29, PO:06:48

Schubert, V. L45, L84, PO:06:18, PO:06:28

Schwanitz, G. L134, L135

Schwarz, T. L190

Schwarz, V. L42

Schwarzacher, T. PO:08:08

Schweizer, D. PO:06:91

Searle, J. L127

Sebastio, L. PO:08:94

Seidel, A. PO:08:69

Serra, L. L129

Seyed Rassaghi, S. PO:06:05

Shahabi, M. PO:06:25

Sham, S. T. PO:08:63

Sharp, H. L137

Shatrova, A. PO:06:20

Shay, J. L69

Sheidai, M. PO:06:85, PO:06:89

Sherman, S. L151

Sherratt, D. L92

Shibata, F. L89, PO:06:21
Shibusawa, M. PO:06:53

Shichiri, M. PO:08:81

Shimada, Y. PO:06:53

Shimizu, R. D. PO:06:07

Shimizu, T. PO:06:08

Shipacheva, O. PO:08:74

Shirayoshi, Y. L125

Shoji, M. L153

Sibson, M. L27

Sidhu, M. C. PO:06:90

Siezen, A. L133

Silahtaroglu, A. L181

Siljak-Yakovlev, S. L59

Simi, P. PO:08:76

Simons, A. PO:06:36

Simpson, K. PO:08:20

Siripatanadilok, S. PO:08:78, PO:08:79

Siroky, J. PO:06:91, PO:08:05

Skaletsky, H. L100

Skalicka, K. PO:06:47

Skalníková, M. PO:06:70

Sklenickova, M. PO:06:62

Slijepcevic, P. L70

Slingsby, T. L178

Slominska, N. L78

Slupphaug, G. L25

Smeets, D. PO:06:36

Smirnova, A. PO:06:63

Smith, J. L09

Smith, M. L116

Solanki, J. V. PO:06:15, PO:06:33

Solovei, I. L76, PO:06:32, PO:06:66, PO:06:71

Soltis, D. PO:06:46

Soltis, P. PO:06:46

Sone, T. L14, L186

Sopariwala, A. PO:08:72, PO:08:90

Sótonyi, P. PO:08:37

Souto, M. PO:08:15, PO:08:16

Specchia, G. PO:08:84, PO:08:94

Speicher, M. PO:06:69

Speicher, M. R. L188

Speleman, F. L142

Spence, J. PO:06:22, PO:08:22

Sperisen, P. L68

Spirito, F. PO:06:55

Srebniak, M. PO:08:82

Staessen, C. L49

Stafne, A. PO:08:55

Stanyon, R. L63

Stein, S. L161, PO:06:32

Stephenson, J. PO:08:83

Stevens, M. R. PO:06:81

Stewart, S. L119

Stodola, T. L119

Stokes, P. L11

Storlazzi, C. T. L172, PO:08:84

Strefford, J. L170

Strombeck, B. L172

Stuessy, T. L59

Sturmey, R. L11 
Suchankova, P. L79, PO:06:83, PO:06:92

Suchkova, I. L78

Suda, T. PO:08:18

Sugiyama, S. PO:08:80, PO:08:81

Sullivan, B. L24

Sullivan, K. L29

Sullivan, S. L74

Sumer, H. L27

Sumner, A. L03

Suputtitada, S. PO:08:79

Surace, C. PO:08:62

Surani, A. L74

Sykorova, E. L184, PO:06:62

Szamalek, J. M. L60

Szeles, A. L144

Sznajer, Y. PO:08:40

Szuhai, K. L141

Tada, T. L153

Tagavi, S. PO:08:73

Taghikhani, M. PO:06:25

Takagi, N. L153

Takao, T. L186

Takata, H. L186, PO:06:08

Takeyasu, K. L191, PO:08:80

Tani, A. PO:06:93

Tanic, N. L15

Tanke, H. L138

Tänzer, S. L60

Tarkan-Argüden, Y. PO:08:51, PO:08:56, PO:08:57, PO:08:77

Tarui, H. L65

Taylor, M. L28

Taylor, S. L22

Tease, C. PO:08:29

Teixeira, T. L68

Telenius, A. L119

Télez, M. PO:08:50, PO:08:52

Teller, K. PO:06:71

Tempest, H. L50

Terauchi, A. PO:06:08

Terriou, P. L102

Tewes, A. PO:06:18

Thanananta, N. PO:08:78, PO:08:79

Thirakhupt, K. PO:06:02

Thomas, A. PO:06:78

Thomas, S. L176

Thorogood, N. L97

Thorsson, A. PO:08:53

Tibshirani, R. L169

Timme, S. PO:06:32

Tinelli, F. PO:08:76

Tjia, W. M. PO:08:63

Tomilin, N. PO:06:63

Tomizuka, K. L125

Tommerup, N. L181

Torricelli, F. PO:06:35, PO:06:61

Toure, A. L101

Trakhtenbrot, L. L157
Traut, W. L105, PO:08:04

Tremetsberger, K. L59

Tsao, M. L139

Tsend-Ayush, E. L106

Tsuchiya, K. PO:06:44, PO:06:49

Turner, J. L101, PO:08:35

Tyson, C. PO:06:30

Uchiyama, S. L14, L83, L186, PO:06:08, PO:06:10

Ugarkovic, D. PO:06:19

Umehara, C. PO:06:52

Umesono, Y. PO:06:52

Uppala, R. PO:06:15, PO:06:15, PO:06:33, PO:06:33

Urien, C. L10

Usatov, A. PO:06:94

Ushiki, T. L156, L182

Uzan, M. PO:08:56, PO:08:57

Vagnarelli, P. L21

Valetto, A. PO:08:76

Valverde, L. PO:08:50

Vamos, E. PO:08:40

Van Assche, E. L49

van Daalen, S. L100

van Dam, M. L154

van der Kraan, I. L37

van Dongen, J. L174

van Driel, R. L37

van Drunen, E. L173

van Erp, F. PO:06:36

van Noort, J. L93

van Ravenswaaij, C. L133, L159

van Roy, N. L142

Van Steirteghem, A. L49

van Zutven, L. L173, L174

Vanderbyl, S. L119

Vandesompele, J. L142

Vankerckhove, S. L46

Varma, N. PO:08:59

Veale, R. PO:08:55

Veble, A. PO:08:12

Velthuizen, S. L173, L174

Veltman, J. L159

Venkataswamy, E. PO:08:10

Venkatesh, N. H. PO:08:10

Verbeeck, J. PO:08:36

Verbrugge, S. L93

Verdoliva, C. PO:06:04

Verheyen, G. L49

Vermeesch, J. PO:08:36

Verschure, P. L37

Vershinin, A. L81, PO:06:56

Vetrie, D. L179

Vidal, F. PO:08:23, PO:08:34

Vignal, A. L09

Visser, A. L29

Vitkova, M. L105, PO:08:04, PO:08:13

Voet, T. PO:08:36

Vogel, J. L130 
Volkov, A. L95

Volkov, R. PO:06:47

Voronin, A. PO:06:59

Vujosevic, M. L15

Vukomanovic, D. PO:08:39

Vyskot, B. PO:08:05

Wade-Martins, R. PO:08:20

Wakayama, T. L125

Wakefield, J. PO:08:03

Wako, T. L82

Walch, A. L146, PO:06:32

Waldor, M. PO:08:02

Walker, L. PO:06:65

Wang, X. L92

Warbuton, D. L155

Ward, D. L04

Wasik-Szczepanek, E. PO:08:88, PO:08:89

Watanabe, N. PO:06:83

Watson, S. L152

Wawrzkiewicz, A. PO:08:82

Webb, A. L178

Weber, B. PO:06:75

Wells, D. L47, L54, PO:06:34

Wenke, T. PO:06:75

Wennekes, J. L88

Werner, M. L146, L161, PO:06:32

Western, P. L74

Whalley, K. L47

White, C. I. PO:06:58, PO:08:24

Wiczkowski, A. PO:08:82

Wiech, T. L146

Wienberg, J. PO:06:01

Wiernik, P. PO:08:45

Willard, H. F. L05, L117

Willem, P. PO:08:03, PO:08:55

Willem Belot, P. PO:08:64

Willemen, J. L159

Wilton, L. L52

Winter, R. L133

Wise, D. $\mathbf{L 1 8 9}$

Witkowska, A. PO:08:93
Wojcierowski, J. PO:08:88, PO:08:89

Wolstenholme, J. L51

Wolvers-Tettero, I. L174

Woroniecka, R. PO:08:93

Wright, A. L91

Wright, W. L69

Writzl, K. L53, PO:08:12

$\mathrm{Wu}$, L. J. L94

Wuite, G. PO:08:01

Xu, Z. L116

Yamada, K. L65 PO:06:44

Yang, F. L41

Yasukochi, Y. L16, PO:08:14

Yates, J. L19

Yilmaz, A. PO:08:51

Yilmaz, S. PO:08:51, PO:08:56, PO:08:57, PO:08:77

Yokokawa, M. L191

Yoneda, A. L83

Yoshido, A. L16, L105, PO:08:14

Yoshimura, S. H. L191

Yoshino, T. PO:08:81

Yosunkaya-Fenerci, E. PO:08:77

Young, B. L177

Yuksel, A. PO:08:77

Zagaria, A. PO:08:84, PO:08:94

Zajac, E. PO:06:16

Zastavna, D. PO:08:67

Zeitlin, S. L25

Zhai, X. L50

Zhdanova, N. PO:06:64

Zieler, H. PO:06:87

Zinner, R. PO:06:32, PO:06:69

Zitzelsberger, H. L146

Zorn, B. L53, PO:08:12

Zou, Y. L69

Zuccotti, M. L44

Zuyderduyn, S. L139 
